\title{
Subjektive Sichtweisen auf Integration und deren Ausgänge in Sportorganisationen
}

\author{
Inauguraldissertation \\ zur Erlangung des Grades eines Doktors der Philosophie \\ im Fachbereich Gesellschaftswissenschaften \\ der Johann-Wolfgang Goethe-Universität \\ zu Frankfurt am Main
}

vorgelegt von

Sarah Weißmann

aus Pirmasens

2022

1. Gutachterin Prof. Dr. Birgit Becker

2. Gutachter Prof. Dr. Michael Fingerle

Datum der Disputation: 26.11.2021 


\section{Inhaltsverzeichnis}

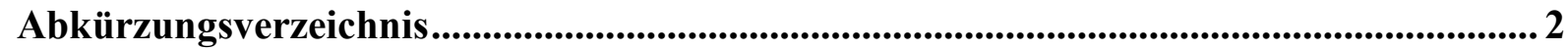

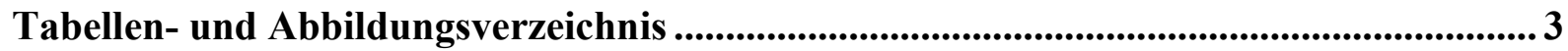

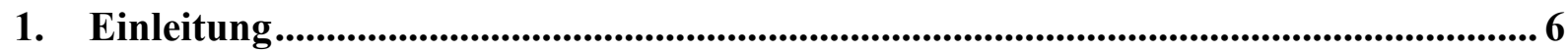

2. Verwendung der Begriffe Integration, Migration und Personen mit Migrationshintergrund ............................................................................................................. 10

2.1 Der Begriff der Integration und Abgrenzung zu anderen Konzepten....................... 10

2.2 Die Begriffe Migration und Personen mit Migrationshintergrund ........................... 15

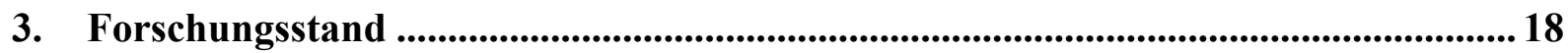

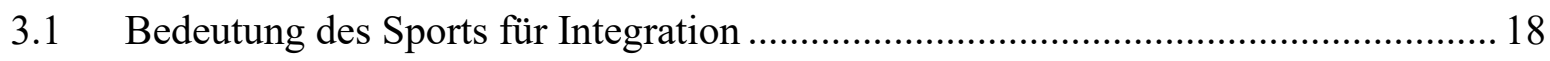

3.1.1 Erwartungen und Maßnahmen in Bezug auf Integration und Sport .................. 18

3.1.2 Einordnung der beschriebenen Programme und Diskussion der Erwartungen 25

3.1.3 Schlussfolgerungen für die eigene Arbeit....................................................... 32

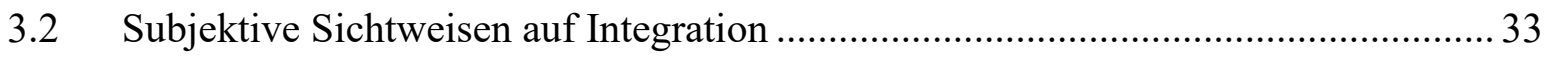

3.2.1 Forschungsarbeiten zu subjektiven Sichtweisen auf Integration ...................... 33

3.2.2 Der Zusammenhang von subjektiven Sichtweisen auf Integration und sozialstrukturellen Merkmalen

3.2.3 Einordnung des Forschungsstandes zu subjektiven Sichtweisen auf Integration..

4 Theoretische Konzepte von Integration ................................................................55

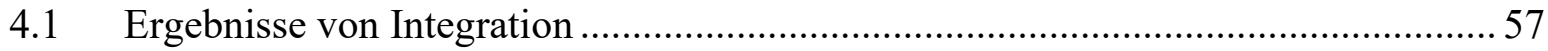

4.1.1 Die Debatte um Assimilation und Pluralismus ............................................... 58

4.1.2 Mehrfachintegration, Assimilation, Marginalität und Segmentation nach

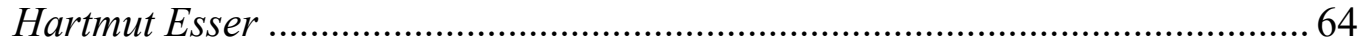

4.1.3 Schlussfolgerungen zu Ergebnissen von Integration für die eigene Arbeit ....... 68

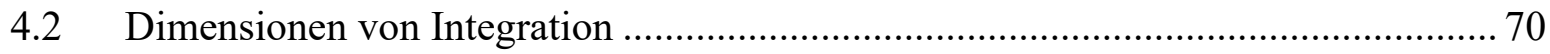

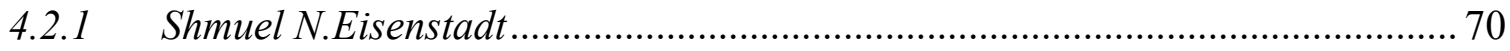

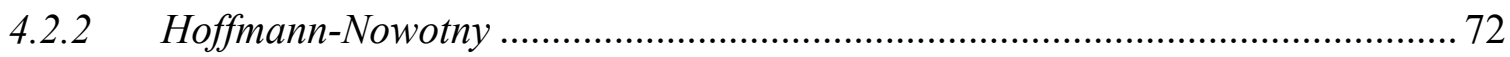

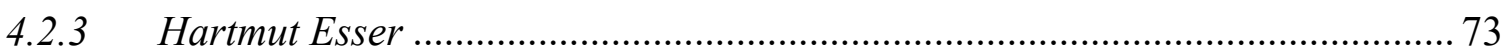


4.2.5 Bielefelder Desintegrationsansatz nach Anhut und Heitmeyer.

4.2.6 Gegenüberstellung der Dimensionen von Integration der theoretischen Konzepte

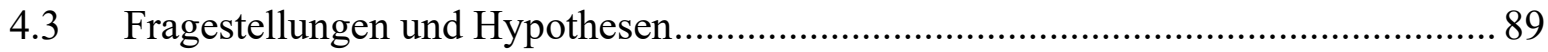

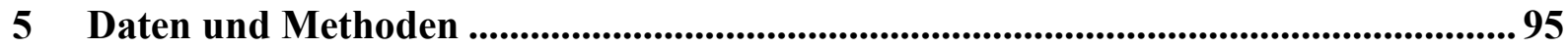

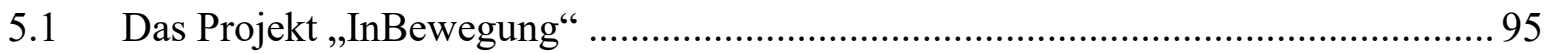

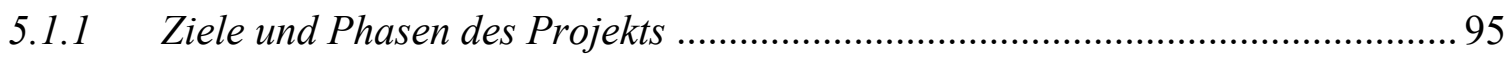

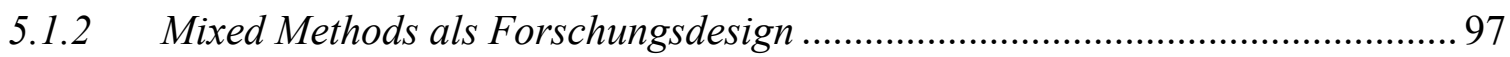

5.1.3 Grundgesamtheit und Stichprobe .............................................................. 100

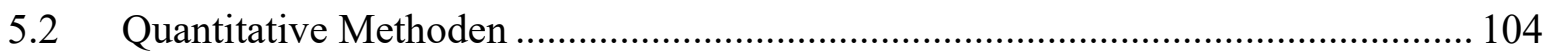

5.2.1 Operationalisierung im Fragebogen ............................................................ 104

5.2.1.1 Wahrgenommene Bedeutung verschiedener Aspekte für Integration .............. 104

5.2.1.2 Einstellungen zu verschiedenen Ausgängen von Integration ........................... 111

5.2.1.3 Sozialstrukturelle Merkmale der Befragten und Merkmale des Sportvereins.. 113

5.2.2 Quantitative Auswertungsmethoden .......................................................... 115

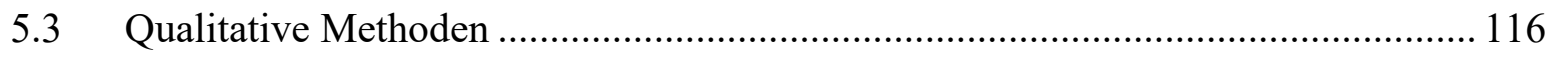

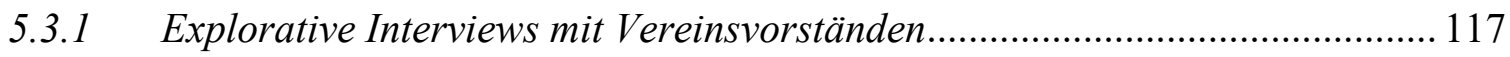

5.3.2 Vertiefende Interviews mit Vereinsmitgliedern ........................................... 118

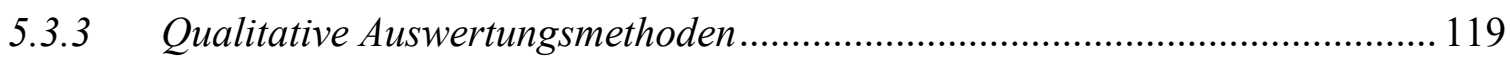

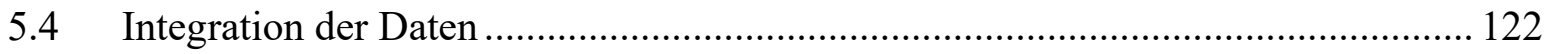

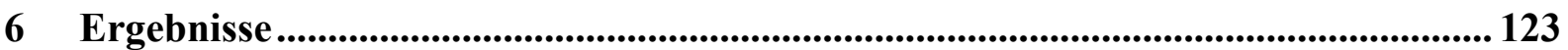

6.1 Quantitative Befragung von Vereinsvorständen und Mitgliedern.......................... 123

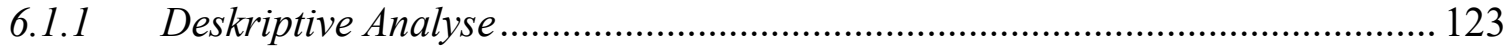

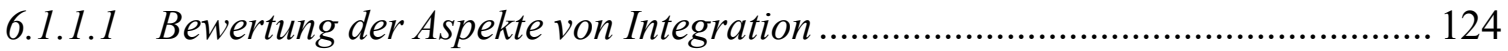

6.1.1.2 Analyse von Unterschieden zwischen Vereinsmitgliedern und Vorständen..... 132

6.1.1.3 Analyse von Unterschieden zwischen Personen mit und ohne Migrationshintergrund 
6.1.2 Zusammenhänge zwischen Ergebnissen und Aspekten von Integration

6.1.3 Zusammenhänge zwischen der Zustimmung zu Integrationskonzepten und sozialstrukturellen Merkmalen der Befragten .............................................. 146

6.1.4 Zusammenfassung und Diskussion der quantitativen Ergebnisse .................... 149

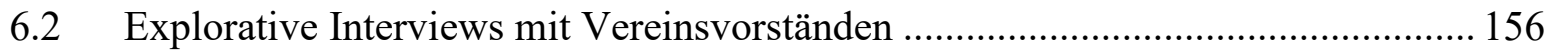

6.2.1 Analyse der Dimensionen von Integration .................................................... 156

6.2.2 Analyse von Ergebnissen sowie Adressatinnen und Adressaten von Integration..

6.2.3 Zusammenfassung und Diskussion der Ergebnisse der explorativen Interviews mit Vereinsvorständen 170

6.3 Vertiefende Leitfaden-Interviews mit Vereinsmitgliedern 173

6.3.1 Unterschiede zu Vereinsvorständen und zwischen Personen mit und ohne Migrationshintergrund 173

6.3.2 Analyse der Dimensionen von Integration..... 174

6.3.3 Analyse von Ergebnissen sowie Adressatinnen und Adressaten von Integration .. 182

6.3.4 Zusammenfassung und Diskussion der Ergebnisse der vertiefenden Interviews mit Vereinsmitgliedern 186

6.4 Zusammenführung der Ergebnisse der unterschiedlichen Datentypen 191

6.4.1 Dimensionen von Integration .....

6.4.2 Ergebnisse sowie Adressatinnen und Adressaten von Integration.

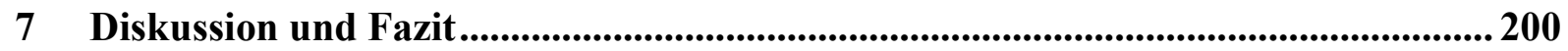

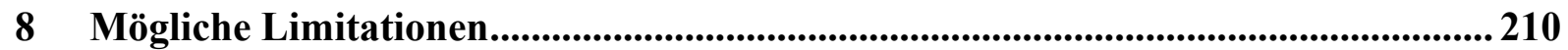

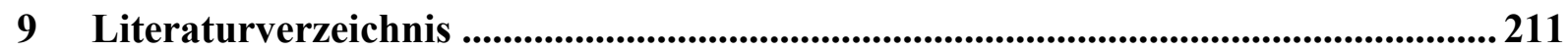

Anhang 222

Danksagung und Lebenslauf Fehler! Textmarke nicht definiert. 
Abkürzungsverzeichnis

AID:A

Bamf

BISP

Bmbf

Bmi

DFB

DOSB

DTB
Aufwachsen in Deutschland: Alltagswelten

Bundesamt für Migration und Flüchtlinge

Bundesinstitut für Sportwissenschaften

Bundesministerium für Bildung und Forschung

Bundesministerium des Innern

Deutscher Fußball Bund

Deutscher Olympischer Sportbund

Deutscher Turner-Bund 


\section{Tabellen- und Abbildungsverzeichnis}

Tabelle 1: Anzahl der Sportvereine in Deutschland in den Jahren 2014 bis 2018 .26

Tabelle 2: Anzahl der Mitglieder in Sportvereinen in Deutschland in den Jahren 2014 bis 2018

Tabelle 3: Typen der (Sozial-) Integration von Migrantinnen und Migranten. 65

Tabelle 4: In dieser Arbeit betrachtete Ausgänge von Integration. 69

Tabelle 5: Gegenüberstellung der Aspekte und Dimensionen von Integration anhand der ausgewählten theoretischen Konzeptionen 83

Tabelle 6: Darstellung der Adressatenebenen der ausgewählten theoretischen Konzeptionen

Tabelle 7: Zusammenfassung der Aspekte zum Oberbegriff Aneignung von Wissen und Werten 89

Tabelle 8: Übersicht des Erkenntnisinteresses der Arbeit. 91

Tabelle 9: Zusammenfassung der Aspekte zum Oberbegriff Aneignung von Wissen und Werten 105

Tabelle 10: Zusammenfassung der Aspekte zum Oberbegriff Kontakte 106

Tabelle 11: Zusammenfassung der Aspekte zum Oberbegriff Zugehörigkeit/Identifikation 107

Tabelle 12: Zusammenfassung der Aspekte zum Oberbegriff Partizipation. 107

Tabelle 13: Zusammenfassung der Aspekte zu den Oberbegriffen Akzeptanz/Anerkennung und Zufriedenheit 108

Tabelle 14: Zusammenfassung der Aspekte zum Oberbegriff Stellung in der Gesellschaft. 109

Tabelle 15: Verwendete Items zur Messung der wahrgenommenen Bedeutung verschiedener Aspekte von Integration 109

Tabelle 16: Werte für Cronbachs Alpha nach unterschiedlichen Dimensionen und Akteursebenen im Gesamtdatensatz.

Tabelle 17: Verwendete Items zur Messung von Einstellungen zu unterschiedlichen Ausgängen von Integration 112

Tabelle 18: Leitfaden für die vertiefenden Interviews 118

Tabelle 19: Kategorien, Beschreibungen und Ankerbeispiele für die qualitative Inhaltsanalyse 119

Tabelle 20: Mittelwerte der Items zu Aspekten von Integration. 128

Tabelle 21: Mittelwerte der Items zu Zielen von Integration 131

Tabelle 22: Ergebnisse der t-Tests und U-Tests zur Bewertung von Aspekten und Zielen von Integration von Vereinsmitgliedern und Vorständen 132 
Tabelle 23: Ergebnisse der t-Tests und U-Tests zur Bewertung von Aspekten und Zielen von Integration von Personen mit und ohne Migrationshintergrund

Tabelle 24: Ergebnisse der t-Tests und U-Tests zur Bewertung von Aspekten und Zielen von Integration von Migrantinnen und Migranten erster und zweiter Generation. 140

Tabelle 25: Zusammenhänge zwischen Ergebnissen von Integration anhand von Spearman's rho.

Tabelle 26: Zusammenhänge zwischen Ergebnissen und Aspekten von Integration anhand von Spearman's rho 145

Tabelle 27: Zusammenhänge zwischen sozialstrukturellen Merkmalen sowie Merkmalen der Sportvereine und der Zustimmung zu Integrationskonzepten anhand von Spearman's rho .. 146 Tabelle 28: Zusammenhänge zwischen Migrationshintergrund und der Zustimmung zu

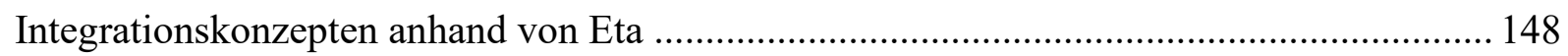

Tabelle 29: Sportvereinen in Hessen in den Jahren 2014 bis 2018 ...................................... 222

Tabelle 30: Mitglieder in Sportvereinen in Hessen in den Jahren 2014 bis 2018 ................. 222

Tabelle 31: Darstellung der Items und Skalen aus der Studie Baur/Mutz 2015 .................. 222

Tabelle 32: Mitgliedschaften und Organisationsgrad unterschiedlicher Altersgruppen in Sportvereinen 2019

Tabelle 33: Test auf Normalverteilung der verwendeten Items zu Aspekten und Ausgängen von Integration

Tabelle 34: Levene-Test auf Varianzhomogenität der Items zu Aspekten und Ausgängen von Integration für Personen mit und ohne Migrationshintergrund. 224

Tabelle 35: Levene-Test auf Varianzhomogenität der Items zu Aspekten und Ausgängen von Integration für Vorstände und Mitglieder 225

Tabelle 36: Levene-Test auf Varianzhomogenität der Items zu Aspekten und Ausgängen von Integration für Migrantinnen und Migranten erster und zweiter Generation 226

Tabelle 37: Leitfaden für die explorativen Interviews 227

Tabelle 38: Ergebnisse des U-Tests der Bewertung von Aspekten und Zielen von Integration von Personen mit europäischem und außereuropäischem Migrationshintergrund 229

Tabelle 39: Zusammenhänge zwischen Aspekten von Integration anhand von Spearman's rho 230

Abbildung 1: Geschätzte Anteile von Personen mit Migrationshintergrund in Sportvereinen 28

Abbildung 2: Phasen des Projekts in Bewegung .... 96 
Abbildung 3: Verteilung der Variable Alter in Prozent ....................................................... 102

Abbildung 4: Verteilung der Variable Bildungsstand in Prozent ........................................ 103

Abbildung 5: Verteilung der Items der Dimension Kulturation in Prozent ......................... 124

Abbildung 6: Verteilung der Items der Dimensionen Interaktion und Identifikation in Prozent

Abbildung 7: Verteilung der Items der Dimension Platzierung in Prozent ......................... 127

Abbildung 8: Verteilung der Items zu Ausgängen von Integration in Prozent...

Abbildung 9: Verteilung des Items Mitgliedschaft/Engagement in Organisationen in Prozent nach Vereinsmitgliedern und Vorständen

Abbildung 10: Verteilung des Items Chancen zur Teilnahme an der Gesellschaft in Prozent nach Migrationshintergrund

Abbildung 11: Verteilung des Items Kontakte zwischen Menschen verschiedener Kulturen in Prozent nach Migrationshintergrund

Abbildung 12: Verteilung des Items Gleiche Rechte für Alle in Prozent nach Migrationshintergrund.

Abbildung 13: Verteilung des Items zu Pluralismus nach Migrationshintergrund

Abbildung 14: Verteilung des Items Kontakte zwischen Menschen verschiedener Kulturen in Prozent nach Migranten erster und zweiter Generation .....

Abbildung 15: Verteilung des Items Vorhandensein eines Zugehörigkeitsgefühls in Prozent nach Migranten erster und zweiter Generation

Abbildung 16: Bedeutung der unterschiedlichen Dimensionen und Aspekte von Integration nach Ansicht der Befragten

Abbildung 17: Anzahl der Kodierungen in den explorativen Interviews mit Vereinsvorständen nach Dimensionen von Integration.

Abbildung 18: Modell der Dimension Interaktion aus Sicht der Vereinsvorstände 160

Abbildung 19: Modell der Dimension Kulturation aus Sicht der Vereinsvorstände 163

Abbildung 20: Zusammenführung der Ausführungen der Vereinsvorstände zu Dimensionen und Aspekten von Integration 171

Abbildung 21: Anzahl der Kodierungen in den Leitfaden-Interviews mit Vereinsmitgliedern nach Dimensionen von Integration.

Abbildung 22: Zusammenführung der Ausführungen von Vereinsvorständen und Mitgliedern zu Dimensionen und Aspekten von Integration aus den Interviews

Abbildung 23: Gesamtbild der subjektiven Sichtweisen auf Dimensionen und Aspekte von Integration aus Erhebung und Interviews...... 


\section{Einleitung}

Der Diskurs über Integration und Integrationsmechanismen fand seinen Beginn in der Migrationsforschung, deren Anfänge bis in die erste Hälfte des 19. Jahrhunderts zurückreichen (Reuter/Mecheril 2015: 1). Innerhalb dieses Feldes fanden immer wieder Wandlungen der Ausgestaltung des Begriffs der Integration statt, da die theoretischen Konzepte in unterschiedlichen gesellschaftlichen sowie historischen Kontexten entstanden sind bzw. entstehen. Heute existiert eine Vielzahl an theoretischen Perspektiven auf Integration, die eine hohe Heterogenität und auch Interdisziplinarität aufweisen. Generell zeigt sich somit, dass, wenn von Integration gesprochen wird, nicht wirklich klar ist, was unter dem Begriff zu verstehen ist. Dazu führt Lutter (2011) aus: „Integration hat als ein zentrales Schlagwort in den gesellschaftspolitischen und fachlichen Diskursen um die Eingliederung von Zuwanderern Aufmerksamkeit erlangt. Was sich hinter der fassettenreichen begrifflichen Formel verbirgt, bleibt jedoch häufig unklar“ (Lutter 2011: 16). Und auch Schramkowksi (2007) beschreibt bezüglich des Integrationsbegriffs, dass dieser sich vor allem durch „Mehrdeutigkeit und Unbestimmtheit"“ auszeichnet (Schramkowksi 2007: 84).

All den unterschiedlichen Konzepten gemein ist dabei, dass es sich um theoretische Annahmen aus dem wissenschaftlichen Diskurs handelt, die sich somit fernab der Lebenswelt von Personen befinden, die als Akteurinnen und Akteure im Integrationsprozess zu sehen sind. Bisherige theoretische Ansätze $\mathrm{zu}$ Integration beschäftigen sich überwiegend mit Interdependenzen und Interaktionen zwischen eingewanderten Personen und der Bevölkerung der Aufnahmegesellschaft, wie beispielsweise innerhalb der Arbeiten von Georg Simmel, Robert Park oder Alfred Schütz. Darüber hinaus beschäftigen sich die sogenannten Zyklenmodelle mit unterschiedlichen Integrationsphasen (Aigner 2017: 14-16). Dabei unterscheiden sich die Ausgestaltungen der Integrationskonzepte und Integration wird anhand unterschiedlicher Phasen, Dimensionen und Aspekte beschrieben.

Eine Perspektive, die im wissenschaftlichen Diskurs weniger Beachtung findet, ist die derjenigen Akteurinnen und Akteure, die in den Integrationsprozess eingebunden sind bzw. einen Teil davon darstellen. Van Dick et al. (1997) führen dazu aus: „Ein Aspekt, der auf die Qualität des Zusammenlebens unmittelbar Einfluss nimmt, ist die individuelle Einstellung der Mitglieder der verschiedenen Gruppen zu Art und Zielen der Koexistenz. Neben anderen Faktoren bestimmen vor allem diese Einstellungen zur multikulturellen Gesellschaft Form, Umfang und Intentionen der Interaktion zwischen den Mitgliedern der verschiedenen Gruppen“ (van Dick et al. 1997: 84). Somit spielt bezüglich der Qualität des Zusammenlebens die 
Einstellung der einzelnen Akteurinnen und Akteure eine bedeutende Rolle, womit im Rahmen der Integration deren Verständnis sowie die Einstellung zu Integration relevant werden, damit diese überhaupt gelingen kann und so in einer guten Qualität des Zusammenlebens mündet. Es stellt sich somit die Frage, was Integration für die Akteurinnen und Akteure im Integrationsprozess bedeutet und welche Einstellung diese zu Integration haben.

Darüber hinaus vermitteln theoretische Ansätze oft den Eindruck, alle Personengruppen hätten das gleiche Verständnis von Integration. Gerade die Sichtweisen von Personen mit Migrationshintergrund werden dabei jedoch außer Acht gelassen. Es wird somit von einem universalen Verständnis von Integration ausgegangen. Gerade bei Personen mit Migrationshintergrund stellt sich jedoch die Frage, ob eine eigene Migrationserfahrung oder die der Eltern die Sichtweisen auf und die Erwartungen an Integration färben, wodurch subjektive Sichtweisen in Bezug auf Integration Relevanz gewinnen. Fragen die sich bezüglich der Sichtweisen der Akteurinnen und Akteure innerhalb dieser Arbeit stellen, sind:

- Welche subjektiven Sichtweisen von Integration (in Form von Verständnis von Integration und Einstellungen zu Integrationskonzepten/Ausgängen von Integration) haben die Akteurinnen und Akteure?

- Welche Dimensionen und Aspekte von Integration sind ihnen dabei wichtig?

- Welche Ergebnisse von Integration werden für wünschenswert gehalten?

- Wie hängen Ergebnisse und Aspekte von Integration zusammen?

- Inwiefern variieren die Sichtweisen auf Integration in Abhängigkeit von sozialstrukturellen Merkmalen und der Zusammensetzung der Mitglieder der Sportvereine?

Die aufgeworfenen Fragen sind für eine Erörterung von subjektiven Sichtweisen auf Integration im Allgemeinen von großem Interesse und sollen in der vorliegenden Arbeit am Beispiel von in Sportvereinen aktiven Personen untersucht werden. Gerade der Sport bietet als favorisierte Organisation in der Freizeitgestaltung, und durch seine Bindungskräfte für Personen mit und ohne Migrationshintergrund, Möglichkeiten des Zusammentreffens und der Interaktion zwischen Personen unterschiedlicher Kulturen. Darüber hinaus kann davon ausgegangen werden, dass gerade auf Ebene der Vorstände und anderer Funktionärinnen und Funktionäre eine gewisse Sensibilisierung für das Thema Integration vorliegt, da im öffentlichen Diskurs spezifische Integrationserwartungen an den Sport formuliert werden und dem Thema Sport auch im Bereich der Integrationspolitik große Bedeutung zukommt. Sportvereine werden außerdem immer wieder als „Integrationsmotor“ bezeichnet und Akteurinnen und Akteure in 
Sportorganisationen somit auch des Öfteren mit dieser Thematik konfrontiert sein. Dies macht Sportvereine zu einem interessanten Untersuchungsgegenstand für subjektive Sichtweisen auf Integration.

Die aufgeworfenen Fragestellungen sollen nicht nur insgesamt, sondern auch auf Ebene von Gruppenvergleichen untersucht werden. Dabei spielt zum einen die Unterscheidung zwischen Vorständen und Vereinsmitgliedern, aber auch zwischen Personen mit und ohne Migrationshintergrund eine Rolle. Die Unterscheidung zwischen Sichtweisen von Vorständen und Vereinsmitgliedern kann innerhalb des Vereins von Bedeutung sein, da Vorstände einen anderen Einfluss auf die Ausgestaltung des Vereins haben als Mitglieder, die oftmals keine aktive Rolle im Verein einnehmen. Baur und Mutz (2015) führen dazu beispielsweise aus, dass die Projekte im Programm „Integration durch Sport“ durch die subjektiven Integrationskonzepte der zuständigen Akteurinnen und Akteure beeinflusst werden (Baur/ Mutz 2015: 155).

In Bezug auf Unterschiede zwischen Personen mit und ohne Migrationshintergrund argumentiert Schramkowksi (2009), dass das Thema Integration in der Öffentlichkeit überwiegend aus der Perspektive der deutschen Mehrheitsgesellschaft dargestellt wird. Darüber hinaus wird „,dabei vor allem über Personen mit Migrationshintergrund gesprochen, anstatt miteinander über Ziele der Integration zu diskutieren und Handlungsstrategien gemeinsam festzulegen“" (Schramkowksi 2009: 149). Dies führt dazu, dass die Sichtweisen von Personen mit Migrationshintergrund überwiegend unberücksichtigt bleiben (Schramkowksi 2009: 149). Und auch in der Fachliteratur existieren keine fundierten Studien, die das Verständnis von Integration aus Perspektive von Eingewanderten oder Personen mit Migrationshintergrund beleuchten (Schramkowksi 2009: 149/150). Demnach stellt die vorliegende Arbeit hinsichtlich des Vergleichs der Sichtweisen von Personen mit und ohne Migrationshintergrund sowie der generellen Abbildung von subjektiven Sichtweisen auf Integration einen Mehrwert dar.

Die wenigen bisherigen Arbeiten zu subjektiven Sichtweisen auf Integration umfassten überwiegend Interviewanalysen (Vgl. Schramkowski 2007 und 2009, Lutter 2011) und keine Vergleiche zwischen Gruppen anhand von quantitativen Daten. Die Abbildung der subjektiven Sichtweisen innerhalb dieser Arbeit umfasst sowohl quantitative als auch qualitative Daten, womit ein Mixed-Methods-Ansatz Verwendung findet. Dadurch können mit Hilfe der quantitativen Daten einerseits Zusammenhänge der Sichtweisen auf Integration mit sozialstrukturellen Merkmalen und Merkmalen der Vereine analysiert werden. Andererseits können die quantitativen Ergebnisse auch durch Interviewanalysen vertieft werden. 
Die vorliegende Arbeit macht es sich somit zur Aufgabe, den Begriff der Integration und damit verbundene Zielvorstellungen aus Sicht der Akteurinnen und Akteure in Sportorganisationen zu beleuchten. Dafür wird eine Studie im Mixed-Methods-Design durchgeführt, die eine fragebogenbasierte Umfrage, explorative Interviews mit Vereinsvorständen und vertiefende Leitfadeninterviews mit Vereinsmitgliedern umfasst. Ein besonderer Fokus der Gruppenvergleiche liegt dabei auf Personen mit und ohne Migrationshintergrund, um die Perspektive von Personen mit Migrationshintergrund selbst in den Diskurs einbringen zu können. Da sich subjektive Sichtweisen auch aufgrund weiterer Faktoren unterscheiden können, werden außerdem Zusammenhänge zwischen sozialstrukturellen Merkmalen sowie Merkmalen der Vereine und subjektiven Sichtweisen auf Integration untersucht.

Die vorliegende Arbeit umfasst insgesamt 8 Kapitel. Im Anschluss an diese Einleitung wird in Kapitel 2 zunächst ein Überblick über die dieser Arbeit zugrundeliegenden Verwendung der Begriffe Integration, Migration sowie Personen mit Migrationshintergrund gegeben. Darauf folgt in Kapitel 3 die Darstellung des Forschungsstandes, der sowohl Ausführungen zur Bedeutung des Sports für Integration als auch zu subjektiven Sichtweisen auf Integration enthält. Kapitel 4 umfasst die in dieser Arbeit verwendeten Konzepte von Integration, die getrennt nach Ergebnissen und Dimensionen von Integration dargestellt werden. Dieses Kapitel schließt mit den aufgeworfenen Fragestellungen und Hypothesen, bevor in Kapitel 5 die Daten, Operationalisierungen und Methoden der Arbeit dargestellt werden. Die Ergebnisse werden in Kapitel 6 vorgestellt. Dabei erfolgt zunächst eine getrennte Darstellung der quantitativen und qualitativen Ergebnisse, welche abschließend zusammengeführt und diskutiert werden. In Kapitel 7 werden die Ergebnisse diskutiert und in einem Fazit zusammengefasst, bevor abschließend in Kapitel 8 Limitationen der vorliegenden Arbeit aufgezeigt werden. 


\section{Verwendung der Begriffe Integration, Migration und Personen mit Migrationshintergrund}

Im Rahmen dieses Kapitels soll zunächst auf die Verwendung der Begriffe Integration, Migration und Personen mit Migrationshintergrund eingegangen werden, da diese nicht unumstritten sind. So wird im nächsten Unterkapitel zunächst auf den Begriff der Integration in Abgrenzung zu anderen Konzepten eingegangen, bevor im folgenden zweiten Unterkapitel die Begriffe Migration und Personen mit Migrationshintergrund sowie deren Verwendung in der vorliegenden Arbeit dargestellt werden.

2.1 Der Begriff der Integration und Abgrenzung zu anderen Konzepten

Im Rahmen dieses Unterkapitels soll, neben einer Erläuterung des verwendeten Begriffs der Integration, eine Abgrenzung zu anderen Konzepten stattfinden sowie dargelegt werden, wieso im Rahmen dieser Arbeit der Begriff der Integration herangezogen wird.

Wie bereits im Rahmen der Einleitung dieser Arbeit dargestellt, weist die Ausgestaltung des Integrationsbegriffs eine hohe Heterogenität und Interdisziplinarität auf.

Im soziologischen Diskurs unterscheiden sich Integrationskonzepte beispielsweise dadurch, ob der Begriff der System- oder Sozialintegration von Relevanz ist. Dabei bezieht sich die Systemintegration auf die Integration „des Systems einer Gesellschaft als Ganzheit, die Sozialintegration dagegen auf die Integration der Akteure (bzw. der von ihnen gebildeten Gruppen) ,in` das System hinein“ (Esser 2001: S. 3) . Außerdem können konsens- oder konflikttheoretische Annahmen zugrunde gelegt werden. Diese Ansichten spiegeln unterschiedliche Strömungen innerhalb der Soziologie wider. Des Weiteren ist eine Unterscheidung in Bezug darauf möglich, ob dem Begriff normative Annahmen zugrunde gelegt werden. Dies ist zum Beispiel dann der Fall, wenn nach einer gelingenden Integration gefragt wird (Burrmann 2014: 22/23) oder danach, welcher Integrationsverlauf erstrebenswert ist (Baur 2009: 108). Im Gegensatz dazu kann der Begriff jedoch auch rein deskriptiv verwendet werden. Dieser Blickwinkel findet in den meisten Fällen Verwendung, wenn tatsächliche Integrationsverläufe von Zuwanderungsgruppen untersucht und unterschiedliche Dimensionen von Integration diskutiert werden (Burrmann 2014: 22/23). Problematisch hierbei ist, dass die Grenzen zwischen normativ wünschenswerten Ergebnissen von Integration und der empirischen Wirklichkeit oft verschwimmen. So wird beispielsweise der Ausgang der

\footnotetext{
${ }^{1}$ Im Rahmen dieser Arbeit ist vor allem die Sozialintegration relevant. Siehe hierzu auch Kapitel 4.
} 
Assimilation in Form der Anpassung als Konzept oft kritisiert, wenn es um normativ wünschenswerte Ergebnisse von Integration geht. Gleichzeitig stellt die Assimilation jedoch einen Ausgang von Integration dar, der empirisch nachgewiesen werden kann ${ }^{2}$.

Im Rahmen eines kurzen Überblicks über die Entwicklungen in der Integrationsforschung wird darüber hinaus deutlich, dass der Begriff der Integration und dessen Ausgestaltung in Bezug auf den Forschungsgegenstand immer wieder Wandlungen unterlag. Diese Wandlungen betreffen zum einen die Perspektive auf Integration und zum anderen die Ausgestaltung des Konzepts selbst in unterschiedlichen Phasen.

Grundlage für die Auseinandersetzung mit Integration und Integrationsmechanismen ist die Migrationsforschung, deren Anfänge bereits bis in die erste Hälfte des 19. Jahrhunderts zurückreichen (Reuter/Mecheril 2015: 1). Zunächst war dieses Forschungsfeld stark auf die Analyse von Mustern und Regeln für Migrationsbewegungen im Allgemeinen fokussiert. Erst Theoretiker wie Georg Simmel, Robert Park oder Alfred Schütz eröffneten eine Perspektive, die vor allem die individuelle Migration sowie Interdependenzen und Interaktionen zwischen den eingewanderten Personen und der Bevölkerung der Aufnahmegesellschaft zum Gegenstand hatte (Aigner 2017: 14). Integrationsmechanismen, im Sinne der Einbindung in eine Gesellschaft, spielten in diesen Ansätzen jedoch weiterhin eine untergeordnete Rolle und erhielten erst in späteren Arbeiten, wie beispielsweise von Park (Chicago School) und Eisenstadt, Bedeutung (Aigner 2017: 15). Diese sogenannten Zyklenmodelle, zu denen auch die Assimilationstheorie von Gordon gehört, beschrieben erstmals unterschiedliche Integrationsphasen und waren dadurch nicht nur auf die Motivbildung zur Migration fokussiert (Aigner 2017: 16).

Heute existiert eine Vielzahl an theoretischen Perspektiven auf Integration. Dies macht es schwierig, in diesem Zusammenhang von einem klaren wissenschaftlichen Feld zu sprechen (Reuter/ Mecheril 2015: 2). Im deutschen Sprachraum sind im Bereich der Migrationssoziologie Hartmut Esser, Hans-Joachim Hoffmann-Nowotny und Friedrich Heckmann als Autoren $\mathrm{zu}$ nennen, die sich überwiegend auf den Aspekt der Integrationsmechanismen beziehen. Die Motivbildung zur Migration hat in diesen Arbeiten hingegen keinerlei Bedeutung (Aigner 2017: 57). Doch nicht nur die dargestellten Perspektiven auf den Forschungsgegenstand unterscheiden sich, auch die Ausgestaltung des Integrationskonzepts selbst weist Unterschiede auf. Im Rahmen der Darstellung dieser

\footnotetext{
${ }^{2}$ Siehe hierzu auch Kapitel 4.1.1. „Die Debatte um Assimilation und Pluralismus“.
} 
Unterschiede bietet es sich an, zwischen Ergebnissen bzw. Ausgängen von Integration und Dimensionen und Aspekten von Integration zu unterscheiden, um eine bessere Übersicht über Entwicklungen im wissenschaftlichen Diskurs zu ermöglichen. Hier zeigt sich im Vergleich unterschiedlicher Konzepte, dass in Bezug auf die Ausgänge von Integration mit der Zeit vom lange herrschenden Assimilationsparadigma Abstand genommen wurde bzw. dieses auch immer wieder starker Kritik ausgesetzt war. Gleichzeitig wurden auch die Dimensionen und Aspekte von Integration immer weiter differenziert und neue Ebenen von Adressatinnen und Adressaten in das Konzept eingeführt. Integration umfasste damit nicht mehr nur Anforderungen an Migrantinnen und Migranten, sondern beispielsweise auch Opportunitäten in der Gesellschaft ${ }^{3}$.

Eine weitere Herausforderung, bei der Betrachtung des Konzepts der Integration, stellt die Abgrenzung zwischen Integration und anderen, neueren Konzepten, wie zum Beispiel Diversitätsmanagement und Inklusion, dar. Hinzu kommen weitere begriffliche Unschärfen durch beispielsweise die synonyme Verwendung des Assimilations- und Integrationsbegriffs (Nobis/Strahle 2009: 119).

Das Konzept des Diversitätsmanagements stammt ursprünglich aus der Wirtschaft und stellt dort ein Steuerungselement zum Umgang mit Vielfalt dar. Ziel dieses Konzepts ist, dass potenzielle Vorteile von Vielfalt durch Unternehmen genutzt werden können. Die Kernkategorien von Diversität sind dabei vielfältig und beziehen sich auf Geschlecht, Alter, Behinderung, Nationalität, Religion und Weltanschauung sowie sexuelle Orientierung. Diese Kernkategorien können darüber hinaus durch weitere Kategorien, die die Partizipation von Individuen betreffen, erweitert werden und sind somit nicht abschließend (Rulofs 2014: 8/9). Generell werden drei Richtungen des Diversitäts-Managements unterschieden. Diese umfassen den Fairness- und Antidiskriminierungsansatz, das Marktzugangs- und Legitimitätsparadigma und den Lern- und Effektivitätsansatz. Der Fairness- und Antidiskriminierungsansatz umfasst dabei eine rechtliche Prägung und stellt im Umgang mit Diversität vor allem auf gesetzliche Vorgaben, wie beispielsweise den Einsatz von Quoten zur gleichberechtigten Teilhabe, ab. Im Zuge des Marktzugangs- und Legitimitätsparadigma wird der besondere Nutzen von Diversität für die Ziele eines Unternehmens betont. Dabei steht jedoch vor allem die Kapitalertragssteigerung im Vordergrund, indem Marktanteile durch die Diversität in einer Organisation gesichert werden. Dieses Paradigma birgt die Gefahr, Minderheiten auf typische Fähigkeiten und einen speziellen Nutzen zu reduzieren. Im Gegensatz stellt der Lern- und

\footnotetext{
${ }^{3}$ Siehe hierzu Kapitel 4 ,Theoretische Konzepte von Integration“.
} 
Effektivitätsansatz, im Rahmen des Diversitätsmanagements, einen langfristigen Lernprozess dar, welcher die Wertschätzung von Vielfalt befördern soll. Die Gefahr der Nutzenorientierung bleibt in diesem Ansatz jedoch ebenfalls bestehen und die Umsetzung ist komplex und anspruchsvoll (Rulofs 2014: 9/10). Darüber hinaus machen die Ausführungen deutlich, dass es sich beim Diversitätsmanagement vor allem um einen Top-Down-Ansatz handelt, der wenig Raum für Mitsprache zulässt. Dies stellt gerade vor dem Hintergrund der starken Fokussierung des Konzepts auf Nutzenmaximierung und Effektivität einen Kritikpunkt dar.

Insgesamt zeigen die Ausführungen auch, dass Diversitätsmanagement auf die Ebene von Organisationen und dabei stark auf Strukturen sowie Gruppenprozesse fokussiert ist. Damit wäre das Konzept auf der Mesoebene anzusiedeln. Es geht dabei nämlich um die Frage, wie Organisationen mit Vielfalt umgehen und auch, wie sie sich für Vielfalt öffnen können. Der Integrationsbegriff kann hingegen, je nach verwendetem Konzept, auf unterschiedlichen Ebenen Anwendung finden. So adressieren Konzepte, die sich mit Systemintegration beschäftigen, die Makroebene, während Konzepte, die Integrationsmechanismen und Interaktionen zwischen Akteurinnen und Akteuren betrachten, auf der Mikroebene anzusiedeln sind (Gieß-Stüber et al. 2014: 37). Im Gegensatz zum Diversitätsmanagement ist der Integrationsbegriff somit, je nach gewähltem Konzept, für vielfältigere Fragestellungen einsetzbar und scheint für diese Arbeit geeignet, da die untersuchten subjektiven Sichtweisen zunächst auf der Mikroebene anzusiedeln sind.

Der Begriff der Inklusion weist sowohl mit dem Ansatz des Diversitätsmanagements als auch mit dem Begriff der Integration Gemeinsamkeiten auf. Betrachtet man zunächst die pädagogische oder auch politische Ausgestaltung des Begriffs, findet dieser seinen Ursprung in der Deklaration von Salamanca und umfasst dort den Anspruch, dass Schulen Kinder unabhängig von psychischen, intellektuellen, sozialen, emotionalen, sprachlichen oder anderen Fähigkeiten aufnehmen (Rulofs 2014: 15). Es handelt sich somit um einen institutionellen Veränderungsprozess zur Ermöglichung von Teilhabe und obwohl der Begriff oft mit Menschen mit Behinderung in Verbindung gebracht wird, kann dennoch auch auf weitere Diversitätsmerkmale abgestellt werden (Rulofs 2014: 16). Somit handelt es sich beim Begriff der Inklusion, wie beim Diversitätsmanagement, um einen Prozess auf der Mesoebene, bei dem mehrere Vielfältigkeitsmerkmale angesprochen werden können. Der Begriff der Integration ist im Gegensatz dazu, gerade durch seinen Zusammenhang mit der Migrationsforschung, stark auf die Kategorie der Herkunft bzw. des Migrationshintergrundes fixiert. Die vorgestellte pädagogische Ausgestaltung des Inklusionsbegriffs entstand darüber hinaus in Abgrenzung zu 
einem assimilativen Integrationsverständnis. Im Rahmen dessen wird ein Individuum in ein bestehendes System aufgenommen, das im ursprünglichen Sinne nicht für dieses konzipiert war (Rulofs 2014: 16). Dass dieses assimilative Integrationsverständnis jedoch nicht das einzige Verständnis von Integration ist, und von diesem über die Zeit auch Abstand genommen wurde, wird im weiteren Verlauf dieser Arbeit noch dargestellt werden ${ }^{4}$.

Im Gegensatz zur pädagogischen Auslegung des Inklusionsbegriffs, zeigen sich in der soziologischen Diskussion Gemeinsamkeiten zwischen Integration und Inklusion. Die Begriffe werden teilweise synonym genutzt oder Inklusion stellt einen Teilbereich oder eine Voraussetzung für Integration dar. So schreibt beispielsweise Esser (2011) „In Hinsicht auf die zweite Frage zeigte sich, daß die so verstandene Inklusion (bzw. Exklusion) nur einen sehr speziellen Fall des gesamten Komplexes der „Integration“ von Migranten und ethnischen Minderheiten abdeckt: die Plazierung der Akteure als eine von vier inhaltlichen Dimensionen der Sozialintegration ... “(Esser 1999: 31). Inklusion beschreibt bei Esser somit eine Dimension von Integration und umfasst im Rahmen der Platzierung „die Besetzung einer bestimmen gesellschaftlichen Position durch einen Akteur“ (Esser 2001: 9).

Dennoch birgt der Begriff der Integration für diese Arbeit den Vorteil, dass er, gerade in der öffentlichen Diskussion, eher mit dem Aspekt des Migrationshintergrundes in Verbindung gebracht wird als andere alternative Konzepte. Da diese Arbeit die subjektiven Sichtweisen in Bezug auf die als wichtig eingeschätzten Aspekte zur Einbindung von Personen mit Migrationshintergrund sowie die Einschätzung zu Ergebnissen in den Blick nimmt, scheint die Verwendung dieses Begriffs zielführend. Eine Verwendung des Inklusionsbegriffs könnte bei den Befragten auch zu Irritationen führen, da dieser eben oft mit dem Aspekt der Behinderung in Verbindung gebracht wird. In Bezug auf den Begriff des Diversitätsmanagements stellt sich die Frage, ob dieser überhaupt einem großen Teil der Bevölkerung bekannt ist. Der Begriff der Integration wird somit für die Beantwortung der aufgeworfenen Forschungsfrage auch als verständlicher für die befragten Personen, und somit als zielführender, eingeschätzt.

Da im Folgenden der theoretische Diskurs zum Integrationsbegriff mit Fokus auf Ausgängen, Dimensionen und Aspekten von Integration dargestellt wird, und die Übereinstimmung mit subjektiven Sichtweisen untersucht werden soll, wird im Rahmen dieser Arbeit zunächst eine weite Definition von Integration herangezogen. Denn eine Untersuchung der subjektiven Sichtweisen auf Integration und damit einhergehend das Verständnis von Integration kann nur stattfinden, wenn diese Sichtweisen nicht von vornherein eingegrenzt werden. Diese weite

\footnotetext{
${ }^{4}$ Siehe dazu Kapitel 4 ,Theoretische Konzepte von Integration“.
} 
Definition des Integrationsbegriffs geht mit seiner ursprünglichen Bedeutung einher, wonach Integration für „,die Vervollständigung eines Ganzen, die Wiederherstellung einer Gesamtheit durch das Einführen ihrer notwendigen Bestandteile in ein Ganzes“ (Soeffner/Zifonun 2005: 404) steht. Integration bezieht sich somit auf einen größeren Gesamtzusammenhang. In Bezug auf Personen mit Migrationshintergrund verweist der Begriff der Integration auf soziale Bezugseinheiten und Gruppen (Lutter 2011: 44). Im Falle dieser Arbeit beziehen sich die eingeführten Bestandteile somit auf Personen mit Migrationshintergrund und die Gesamtheit bezeichnet die Gesellschaft, in der die entsprechende Person lebt. Insgesamt beziehen sich subjektive Sichtweisen auf Integration somit auf alle Sichtweisen, die den Einbezug von Personen mit Migrationshintergrund in eine Gesellschaft umfassen.

\subsection{Die Begriffe Migration und Personen mit Migrationshintergrund}

Um den Begriff der Personen mit Migrationshintergrund $\mathrm{zu}$ definieren, wird in diesem Unterkapitel zunächst auf Definitionen von Migration eingegangen, um anschließend die Verwendung des Begriffs Personen mit Migrationshintergrund im Rahmen dieser Arbeit darzustellen.

Im Allgemeinen wird unter Migration die räumliche Verlegung des Lebensmittelpunkts einer Person verstanden (BAMF 2015: 30). Oswald definiert Migration als „Prozess der räumlichen Versetzung des Lebensmittelpunktes, also einiger bis aller relevanten Lebensbereiche an einen anderen Ort, der mit der Erfahrung sozialer, politischer und/oder kultureller Grenzziehung einhergeht" (Oswald 2007: 13). Zusammen mit einem Ortswechsel kommt es somit auch zu einer außerordentlichen psycho-sozialen Leistung (Oswald 2007: 14).

Um die vielfältigen Formen der Mobilität von Migration abzugrenzen, werden in der Literatur auch die Dimensionen Zeit, Entfernung, Charakter der Entscheidung, Zweck und Akteurin bzw. Akteur unterschieden, wenn es um den Begriff der Migration geht. So sehen manche Autorinnen und Autoren nur die dauerhafte Verlegung des Wohnsitzes als Migration an und andere wiederrum ziehen bestimmte Zeitgrenzen in Betracht. Eine besondere Rolle spielt auch die Dimension der Entfernung. Hier stellt sich die Frage, ob eine bestimmte räumliche Entfernung vorhanden sein muss, oder ob beispielsweise auch die kulturelle Ferne bereits eine Rolle spielt. Betrachtet man den Begriff der Migration aus einer politischen Perspektive, wird zwischen der Migration innerhalb eines Staates (Binnenmigration) und der grenzüberschreitenden Migration unterschieden. Dabei kann auch der Begriff der Grenze unterschiedlich ausgelegt werden, nämlich als nationale oder innerstaatliche bzw. 
administrative Grenze. In den Politikwissenschaften sowie der Mikrosoziologie ist jedoch die Definition der Grenze als nationale Grenze üblich, während innerstaatliche bzw. administrative Grenzen eher in der Demographie eine Rolle spielen. Darüber hinaus kann aber auch die Entscheidung zu migrieren eine Rolle spielen. In diesem Fall ist zwischen erzwungener und freiwilliger Migration zu unterscheiden.

Innerhalb des Zwecks der Migration kann zwischen Reisen, Tourismus und tatsächlicher Migration unterschieden werden. Auch innerhalb dieser Dimension kommt es zu Diskussionen, da teilweise bereits längere Reisen als temporäre Migration angesehen werden. Im Rahmen der letzten Dimension, der der Akteurin bzw. des Akteurs, wird auf die aktive Willensentscheidung der migrierenden Person abgestellt (Düvell 2006: 7-10).

Im Rahmen dieser Arbeit ist die politische Dimension des Migrationsbegriffs von Bedeutung. Es geht somit um die Überschreitung nationaler Grenzen und die damit einhergehende Verlegung des Lebensmittelpunktes in einen anderen Staat. Gleichzeitig werden in dieser Arbeit jedoch nicht nur Personen mit eigener Migrationserfahrung betrachtet, sondern auch deren Nachkommen. Aus diesem Grund wird der Begriff Personen mit Migrationshintergrund herangezogen.

Auch in Bezug auf die Verwendung des Begriffs Migrationshintergrund existieren unterschiedliche Ansätze. In Deutschland umfassen die am häufigsten verwendeten Indikatoren den Geburtsort oder die Staatsangehörigkeit der Person und/oder ihrer Eltern bzw. Großeltern, den Sprachhintergrund bzw. Sprachgebrauch sowie das spezifische Geburtsland. Im Rahmen des Geburtsortes wird dabei überwiegend nur zwischen Inland und Ausland sowie bei der Staatsangehörigkeit lediglich zwischen deutscher und ausländischer Nationalität unterschieden. Insgesamt zeigt sich, dass Indikatoren für die Feststellung des Migrationshintergrundes somit subjektiver oder objektiver Natur sein können (Maehler et al. 2016: 264).

Der Begriff Personen mit Migrationshintergrund bezieht sich in dieser Arbeit auf Zugewanderte und deren Nachkommen. Somit fallen unter diesen Begriff nicht nur im Inland lebende Ausländerinnen und Ausländer, sondern auch eingebürgerte Einwandererinnen und Einwanderer sowie ihre Nachkommen. Der entscheidende Indikator für die Feststellung eines Migrationshintergrundes ist somit der Geburtsort der Person und der Eltern. Die Staatsbürgerschaft wird in der vorliegenden Arbeit nicht als Abgrenzungsmerkmal herangezogen, da Staatsbürgerschaften über den Lebensverlauf erworben, aufgegeben oder verloren werden können und daher nicht immer Stabilität aufweisen (Petschel/Will 2020: 81). Darüber hinaus stellen die in der Arbeit vorgestellten theoretischen Arbeiten zu Integration 
überwiegend auf Zugewanderte ab, wodurch die Verwendung einer wanderungsbezogenen Definition des Migrationshintergrundes bevorzugt werden sollte.

Durch die Unterscheidung zwischen Geburtsort der Person und der Eltern kann ein Migrationshintergrund erster oder zweiter Generation vorliegen. Die Unterscheidung zwischen erster und zweiter Generation ist wie folgt definiert: Ist eine eigene Migrationserfahrung vorhanden, handelt es sich um Migrantinnen und Migranten erster Generation. Die Nachkommen der Migrantinnen und Migranten erster Generation, welche im Zuwanderungsland geboren wurden und somit keine Migrationserfahrung besitzen, werden als Migrantinnen und Migranten zweiter Generation bezeichnet (BAMF 2015: 214). Innerhalb der Gruppe der Migrantinnen und Migranten zweiter Generation kann darüber hinaus danach unterschieden werden, ob beide Eltern im Ausland geboren wurden (,foreign background“), oder ob mindestens ein Elternteil im Ausland geboren wurde (,mixed background“) (Eurostat 2016). Die erste und zweite Generation werden in dieser Arbeit unter dem Begriff Personen mit Migrationshintergrund zusammengefasst.

Im theoretischen Rahmen sowie im Forschungsstand dieser Arbeit wird teilweise der Begriff Zugewanderte oder Migrantinnen und Migranten beibehalten, sofern sich die theoretischen Ansätze oder Forschungsarbeiten nur auf Personen mit eigener Migrationserfahrung beziehen. 


\section{Forschungsstand}

Im Rahmen dieses Kapitels soll zum einen die Relevanz des Sports für Integration sowie der für diese Arbeit wesentliche Forschungsstand dargestellt werden. Dazu wird zunächst auf die Bedeutung des Sports für Integration eingegangen. Diese Bedeutung zeigt sich nicht nur durch Forschungsarbeiten zu diesem Thema, sondern auch durch Präsenz des Themas auf politischer Ebene.

Im darauffolgenden Unterkapitel wird anschließend der Forschungsstand zu subjektiven Sichtweisen auf Integration dargestellt. Dabei sind nicht nur Arbeiten, die sich explizit mit Sportorganisationen beschäftigen von Bedeutung, sondern auch Arbeiten, die diese Thematik in anderen Bereichen betrachten.

\subsection{Bedeutung des Sports für Integration}

In diesem Unterkapitel werden zunächst Erwartungen und Maßnahmen in Bezug auf Integration im Sport exemplarisch durch die Darstellung des Themas im Integrationsplan der Bundesrepublik Deutschland, in der Integrationspolitik der hessischen Landesregierung und im Projekt „Integration durch Sport“ des Deutschen Olympischen Sportbundes (DOSB) aufgezeigt. Durch die Auswahl dieser drei exemplarischen Programme, soll die Bedeutung des Themas auf unterschiedlichen politischen Ebenen aufgezeigt werden. Im Anschluss daran erfolgt eine Einordnung der beschriebenen Programme sowie eine Erörterung darüber, inwieweit sich die Erwartungen an den Sport als „Integrationsmotor“ auch in wissenschaftlichen Untersuchungen widerspiegeln. Das Unterkapitel schließt mit einer kurzen Zusammenfassung in Form von Schlussfolgerungen für die eigene Arbeit ab.

\subsubsection{Erwartungen und Maßnahmen in Bezug auf Integration und Sport}

Sport spielt auf unterschiedlichen politischen Ebenen eine Rolle für Integration. So taucht das Thema im Nationalen Integrationsplan auf und erhält dort sogar ein eigenes Kapitel, das dem Umfang der Abschnitte zu Bildung und Spracherwerb gleicht (Mutz 2018: 11). Aber auch die hessische Landesregierung und der DOSB beschäftigen sich mit dieser Thematik.

Im Nationalen Integrationsplan werden Integrationschancen im Sport auf den Ebenen der sozialen, kulturellen und alltagspolitischen Integration gesehen. Soziale Integration findet dabei dadurch statt, dass Personen aus unterschiedlichen Nationen oder Kulturen miteinander in Kontakt kommen und soziale Beziehungen aufbauen. Die kulturelle Integration erfolgt durch 
die Vermittlung bestimmter Kulturtechniken wie Sprache oder Verhaltensmuster im Alltag. Außerdem kommt es durch die Mitsprache und das Engagement in den Sportorganisationen zu einer alltagspolitischen Integration.

Rahmenbedingungen für eine erfolgreiche Integration im Sport sind, laut Integrationsplan, die Entwicklung zielgruppenorientierter und nachhaltiger Angebote, die Stärkung interkultureller Vermittlerinnen und Vermittler sowie die Sicht auf Integration als wechselseitiger Prozess. Gleichzeitig wird jedoch auch festgestellt, dass aufgrund der Heterogenität der Vereine keine Verallgemeinerung der Rahmenbedingungen erfolgen sollte (Bundesregierung 2007: 140). Zielbestimmungen des Integrationsplan im Bereich Integration im Sport sind die Dokumentation und Evaluation von Projekterfahrungen, Kompetenzerweiterung und Qualifizierung, Kooperation in lokalen Netzwerken, Schaffung leistungsfähiger Rahmenbedingungen für Integrationsarbeit sowie die interne und externe Stärkung des Themas Integration. Dazu sollen, im Rahmen der Dokumentation und Evaluation von Projekterfahrungen, die Erfahrungen aus positiv verlaufenen Projekten dokumentiert und vernetzt sowie auf ihre Übertragbarkeit untersucht werden. Die Kompetenzerweiterung und Qualifizierung bezieht sich auf die Identifikation von Erfolgsfaktoren für Integration und die Sensibilisierung der Personen in Sportorganisationen für das Thema. Darüber hinaus sollen Schulungsbausteine entwickelt werden, die vor allem den Bereich interkulturelle Kompetenz fördern.

Kooperationen in lokalen Netzwerken sind laut des Integrationsplans erforderlich, um die Integrationspotenziale des Sports in vollem Umfang nutzen zu können. Denn Kooperationen fördern das Verständnis füreinander und können auch in Kooperationsprojekten münden. Dabei ist es von besonderer Bedeutung, auch eigenethnische Vereine mehr in die Netzwerke einzubinden.

Ein weiterer Punkt ist die Schaffung leistungsfähiger Rahmenbedingungen für die Integrationsarbeit. Um Integrationskonzepte entwickeln zu können, müssen Beratungsangebote vorhanden sein, die Vereine mit weniger Ressourcen und Kenntnissen bestmöglich unterstützen. Von besonderer Bedeutung sind dabei auch die Bereitstellung von Sportstätten sowie „die Schaffung von Bedingungen, die den kulturellen, sozialen und religiösen Erfordernissen von Mädchen und Frauen mit Migrationshintergrund entsprechen“ (Bundesregierung 2007: 141).

Die letzte Zielbestimmung des Integrationsplans ist die interne und externe Stärkung des Themenbereichs insgesamt. Aus diesem Grund soll das Thema auch in den zuständigen 
Behörden und betroffenen Institutionen angesiedelt sein (Bundesregierung 2007: 141). Darüber hinaus sind breite Öffentlichkeitsarbeit in diesem Bereich und die systematische Einbindung von Personen mit Migrationshintergrund in die Kommunikationsstrukturen von Bedeutung (Bundesregierung 2007: 141/142).

Für die Förderung der vorgestellten Zielbestimmungen stellt der Integrationsplan Maßnahmen der Bundesregierung, Länder und Kommunen sowie nicht staatlicher Organisationen und Institutionen dar. Die Maßnahmen der Bundesregierung umfassen dabei unter anderem die Einrichtung einer Informationsplattform als Grundlage zur Erfassung von Integrationsangeboten im Sport, eine Evaluation des Programms „Integration durch Sport“, die Verankerung des Themas als Forschungsschwerpunkt beim Bundesinstitut für Sportwissenschaften (BISP) und die Weiterentwicklung des Programms „Integration durch Sport“ (Bundesregierung 2007: 142). Länder und Kommunen „sind neben den kommunalen Gebietskörperschaften - insbesondere im Breitensport - die Hauptlastenträger u. a. beim Bau, der Sanierung und der Modernisierung von Sportstätten. Darüber hinaus fördern die Länder häufig einzelne Pilot- oder Modellprojekte, um den Integrationsgedanken vor Ort stärker greifen zu lassen“ (Bundesregierung 2007: 142). Da eine Aufzählung aller Maßnahmen der nicht-staatlichen Organisationen und Institutionen nicht möglich ist, werden im Integrationsplan stellvertretend die Maßnahmen des Deutschen Olympischen Sportbundes (DOSB), Deutschen Fußball-Bundes (DFB) und Deutschen Turner-Bundes (DTB) aufgeführt.

Im ersten Fortschrittsbericht zum nationalen Integrationsplan (2008) wird von der Einrichtung einer ständigen Arbeitsgruppe „Integration und Sport“ berichtet, welche Empfehlungsstrukturen auf Bundesebene erarbeitet und weitere Schwerpunkte an der Schnittstelle Integration und Sport identifizieren soll. Darüber hinaus soll die interkulturelle Kompetenz der Akteurinnen und Akteure vor Ort durch Handreichungen gefördert, bestehende Angebote ausgebaut und Netzwerke erweitert werden (Bundesregierung 2008: 29).

Im Jahr 2011 kam es zu einer Weiterentwicklung des Integrationsplans im Rahmen des Nationalen Aktionsplans Integration. Auch hier erhält das Thema Sport ein eigenes Kapitel. Im Bereich der Zielbestimmungen wird dabei zwischen strategischen und operativen Zielen unterschieden. Zu den strategischen Zielen des Aktionsplans gehören zum einen die Integration in den Sport und zum anderen die Integration durch den Sport. Es wird somit nun zwischen diesen beiden Dimensionen unterschieden, wobei auch festgestellt wird, dass sich diese Dimensionen nicht immer trennscharf voneinander unterscheiden lassen (Bundesregierung 2011: 247/248). 
Die Integration in den Sport bezieht sich auf Personen, die bisher im vereinsorganisierten Sport unterrepräsentiert sind. „Dies sind zum Beispiel Mädchen und Frauen, ältere Erwachsene sowie sozial Benachteiligte. Zielgruppe des Forums sind aber vor allem Menschen mit Migrationshintergrund, die im Vergleich zum Bevölkerungsdurchschnitt weiterhin deutlich unterrepräsentiert sind“ (Bundesregierung 2011: 248). Im Gegensatz dazu bezieht sich die Integration durch den Sport auf die Nutzung der Integrationspotenziale in Vereinen. Dabei spielen vor allem Multiplikatorinnen und Multiplikatoren mit und ohne Migrationshintergrund in den Vereinen eine wichtige Rolle (Bundesregierung 2011: 248).

Die operativen Ziele des Aktionsplans im Bereich Integration in den Sport umfassen insgesamt neun Punkte, die durch Maßnahmenbeispiele ergänzt werden (Bundesregierung 2011: 249/250). Diese Punkte sind die Förderung der Teilhabemöglichkeiten, die Annäherung des Anteils von Mädchen/Frauen und Jungen/Männern bei Mitgliedern sowie bei Funktionärinnen und Funktionären, die Stärkung von interkulturellen Kompetenzen der Funktionärinnen und Funktionäre sowie der Mitarbeitenden der kommunalen Verwaltung und Netzwerke vor Ort, eine Zielgruppenerweiterung mit spezifischen Sportangeboten, die Nutzung von Synergieeffekten vor Ort, der Einbezug von Migrantinnen- und Migrantenvereinen in die Integrationsverantwortung, die Verankerung des Themas Integration in Verbands- und Vereinsstrukturen, die Ausweitung der Integration sozial Benachteiligter und der Ausbau sportwissenschaftlicher Forschung.

Für das strategische Ziel Integration durch den Sport sieht der Aktionsplan folgende sechs Maßnahmen vor: Förderung von praxisnaher Sprachanwendung und Bildung durch Sport, Nutzung des Sozialisierungspotenzials des Sports, Stärkung des Praxisbezugs sportwissenschaftlicher Forschung, Verbindung von Sport und gesundheitlicher Aufklärung, Bewerbung und Bereitstellung von passgenauen Angeboten für Seniorinnen und Senioren mit und ohne Migrationshintergrund, Nutzung von Sportvereinen als niedrigschwellige Job- und Kontaktbörsen (Bundesregierung 2011: 252).

Im Vergleich zum Integrationsplan aus dem Jahr 2007 zeigt sich im Nationalen Aktionsplan Integration ein konkreteres Bild der Ziele im Bereich Sport und Integration. Außerdem verdeutlichen einzelne Beispiele aus der Praxis mögliche Maßnahmen im Bereich der Zielvorstellungen. Darüber hinaus werden die Ziele spezifisch für die Bereiche Integration in den Sport und Integration durch den Sport formuliert, was eine Konkretisierung einzelner Ziele ermöglicht. 
Im Jahr 2020 wurden im Rahmen des 10. Integrationsgipfels fünf Phasen der Integration herausgearbeitet, mit deren Hilfe Handlungsfelder und konkrete Maßnahmen abgeleitet werden sollen. Auch hier spielt Sport in der vierten Phase, der „Phase des Zusammenwachsens Vielfalt gestalten - Einheit sichern“, eine Rolle. Weitere Informationen zur Ausgestaltung liegen jedoch noch nicht vor (Bundesregierung o.J.).

Auch die hessische Landesregierung beschäftigt sich in ihrer Veröffentlichung „Integrationspolitik der Hessischen Landesregierung“ (2012) mit dem Thema Sport. Im Rahmen des Nationalen Aktionsplans Integration sind in Hessen einige Projekte initiiert worden, die die Integration im Bereich Sport fördern sollen. „Um die [oben genannten] Ziele $\mathrm{zu}$ erreichen, ist die Landesregierung bereits in unterschiedlichen Tätigkeitsfeldern aktiv. Neben der Gewinnung zusätzlicher Menschen mit Migrationshintergrund (insbesondere auch von Kindern und Jugendlichen) für eine Mitgliedschaft oder ehrenamtliche Tätigkeit in Sportvereinen engagiert sie sich bei der Förderung des Wettkampf- und Leistungssports (insbesondere im Nachwuchsbereich), des gesellschaftlichen Austauschs mit Migrantenorganisationen, der Aus-, Fort- und Weiterbildung von Übungsleiterinnen und Übungsleitern, Trainerinnen und Trainern sowie Betreuerinnen und Betreuer mit Migrationshintergrund, der Gewaltprävention im Sport, aber auch der Verbesserung der Sprachkenntnisse und der Gesundheitsförderung“ (Hessisches Ministerium der Justiz, für Integration und Europa 2012: 49/50). Projekte der hessischen Landesregierung sind beispielsweise „START - Sport überspringt kulturelle Hürden“, „Fußball ist das Tor zum Lernen“, das „Ballance-Projekt“ und „Fußball trifft Kultur“.

Im Landesprogramm „Modellregionen Integration“ finden sich konkrete Handlungsempfehlungen der hessischen Landesregierung zur Integration in und durch den Sport. Diese Handlungsempfehlungen wurden im Rahmen der wissenschaftlichen Begleitung des Landesprogramms erarbeitet. Insgesamt umfassen diese Empfehlungen die Schaffung niedrigschwelliger und offener Angebote, die Anpassung bestehender Angebote an die neue Zielgruppe - beispielsweise durch die Qualifizierung von Personen mit Migrationshintergrund als Übungsleiterinnen und Übungsleiter, die Durchführung gezielter integrationsfördernder Maßnahmen sowie die Schaffung einer Willkommenskultur durch Abbau von Zugangsbarrieren durch zum Beispiel die Bereitstellung von Informationsmaterialien in verschiedenen Sprachen (Hessisches Ministerium der Justiz, für Integration und Europa 2013: 153). Darüber hinaus sollen für eine breitere Vernetzung lokale und regionale Angebote in Form eines Überblicks zusammengetragen werden und auch eigenethnische Vereine in die 
Schaffung neuer Angebote einbezogen werden (Hessisches Ministerium der Justiz, für Integration und Europa 2013: 156).

Neben diesen Erwartungen und Maßnahmen auf bundes- und landespolitischer Ebene beschäftigt sich auch der Deutsche Olympische Sportbund (DOSB), unter anderem im Rahmen des Programms „Integration durch Sport“, mit den Integrationspotenzialen des Sports.

Das Programm besteht bereits seit 1981 und wurde damals unter dem Namen „Sport für Alle Sport mit Aussiedlern“ in den Ländern Niedersachsen, Berlin, Nordrhein-Westfalen und Hessen implementiert. Im Jahr 1991 wurde das Programm auf alle Bundesländer ausgeweitet und 2001 aufgrund der Zuwanderungssituation auf Migrantinnen und Migranten als Zielgruppe erweitert. Somit entstand das Programm „Integration durch Sport“, welches durch das Bundesministerium des Innern (BMI) und das Bundesamt für Migration und Flüchtlinge (BAMF) gefördert und durch die Landessportbünde getragen wird. Die Koordination auf Bundesebene obliegt dem DOSB (DOSB 2014a: 4). Der DOSB sieht im organisierten Sport ein besonderes Integrationspotential. Dieses wird zum einen begründet durch die flächendeckende Verbreitung der Vereine. Sportvereine sind somit für fast alle Menschen erreichbar. Darüber hinaus stellen Sportvereine einen beliebten Ort der Freizeitbeschäftigung und Sport im Allgemeinen ein kultur- und schichtübergreifendes Phänomen dar. Zum anderen besitzen Sportvereine eine politische sowie religiöse Neutralität, die sie für alle öffnet. Die Regeln sind universell und durch nonverbale Kommunikation können auch Sprachprobleme überwunden werden (DOSB 2014b: 17).

Damit diese Integrationspotenziale genutzt werden können, werden im Programm gemeinsame Integrationsziele definiert, die Spielraum für eine Anpassung an die länderspezifischen Rahmenbedingungen lassen. Diese gemeinsamen Integrationsziele umfassen folgende Punkte:

- Gleichberechtigte Teilhabe der Zielgruppen auf allen Ebenen

- Integration durch Sport in die Gesellschaft

- Förderung und Unterstützung der Sportorganisationen bei der Integrationsarbeit

- Förderung und Anerkennung des freiwilligen Engagements

- Förderung der interkulturellen Öffnung des Sports

- Stärkung des Themas Integration in den Strukturen des Sports

- Kontinuierliche Weiterentwicklung und Optimierung des Programms (DOSB 2014a: 7/8). Alle Integrationsziele werden weiter mit bestimmten Maßnahmen beschrieben. Die gleichberechtige Teilhabe der Zielgruppen auf allen Ebenen umfasst vor allem Information, 
aktive Ansprache, zielgruppenspezifische Angebote sowie die Minderung von Zugangsbarrieren. Für eine Integration durch Sport in die Gesellschaft sollen Situationen und Anlässe geschaffen werden, die weiterreichende Integrationsprozesse in die Gesellschaft in Gang setzen. „Das sportliche und außersportliche Vereinsleben bietet vielerlei situationsgebundene Anlässe, in denen kulturelle Konventionen, Alltagswissen oder ein vertiefender Spracherwerb quasi nebenbei vermittelt werden“ (DOSB 2014a: 7). Darüber hinaus bergen Sportvereine auch Möglichkeiten zur demokratischen Mitsprache. Diese Aspekte können im Sportverein somit erlernt und auf andere gesellschaftliche Kontexte übertragen werden (DOSB 2014a: 7).

Weitere Ziele sind die Förderung und Unterstützung der Sportorganisationen bei der Integrationsarbeit, Förderung und Anerkennung des freiwilligen Engagements sowie die Förderung der interkulturellen Öffnung des Sports. Im Rahmen der Unterstützung der Sportorganisationen stehen dabei finanzielle Hilfe und professionelle Beratung im Mittelpunkt, da viele Vereine nicht über ausreichend Ressourcen und auch Kenntnisse im Bereich der Integration verfügen. Für die Förderung des freiwilligen Engagements sollen vor allem niedrigschwellige Formen des Engagements geschaffen und Migrantinnen und Migranten bei der Übernahme von Ämtern unterstützt werden. Das Ziel der interkulturellen Öffnung des Sports spielt insgesamt eine übergeordnete Rolle, da sie als „unverzichtbarer Bestandteil der Integrationsarbeit im Sport“ zu sehen ist (DOSB 2014a: 8). Hier spielen vor allem die Vermittlung interkultureller Kompetenz sowie eine stärkere Repräsentanz von Migrantinnen und Migranten sowie eine Sensibilisierung der Akteurinnen und Akteure für das Thema eine Rolle.

Die letzten beiden formulierten Ziele des Programms sind die Stärkung des Themas Integration in den Strukturen des Sports und die kontinuierliche Weiterentwicklung und Optimierung des Programms. Für die Stärkung des Themas Integration in den Strukturen des Sportkreises wird auf die Wirksamkeit von ehrenamtlichen Integrationsbeauftragten verwiesen. Außerdem sollen Vereine ihre Integrationsarbeit mit Öffentlichkeitsarbeit begleiten. „Diese [Öffentlichkeitsarbeit] soll gleichermaßen die einheimische wie die zugewanderte Bevölkerung erreichen und die Leistungen des Sports in der Integrationsarbeit positiv hervorheben“ (DOSB 2014a: 8). Eine Weiterentwicklung und Optimierung des Programms beinhalten vor allem das Zusammentragen, Dokumentieren, Bewerten und Vernetzen von erfolgreichen Projekten und Initiativen. Denn „,der Sport selbst [kann] mit seinem innovativen und vielschichtigen Potenzial 
Impulse setzen und eine tragende Rolle im gesamtgesellschaftlichen Integrationsprozess übernehmen“(DOSB 2014a: 8).

\subsubsection{Einordnung der beschriebenen Programme und Diskussion der Erwartungen} Insgesamt zeigt sich im Rahmen der Beschreibung der Programme, dass die Bedeutung des Sports für Integration auf den vorgestellten Ebenen schon seit einiger Zeit präsent ist und dem Sport dabei eine große Bedeutung zugewiesen wird. Weiter ist bei der Betrachtung des Themas Integration und Sport auf den Ebenen Bund, Land Hessen und des DOSB auch zu erkennen, dass auf allen Ebenen sowohl Integrationsziele als auch spezifische Maßnahmen im Bereich des vereinsorganisierten Sports formuliert werden. Dies zeigt, dass die Integration im Sport kein Selbstläufer ist, Sport also nicht automatisch integrierend wirkt, und somit die Unterstützung der Sportorganisationen mit bestimmten Maßnahmen notwendig wird. Diese Maßnahmen werden im Nationalen Integrationsplan auf den Ebenen Bundesregierung, Länder und Kommunen sowie nicht-staatliche Organisationen und Institutionen beschrieben. Auch in der hessischen Integrationspolitik zeigt sich, dass die integrative Wirkung des Sports nur genutzt werden kann, wenn aktiv auf Personen mit Migrationshintergrund zugegangen wird und ein geeignetes Umfeld für die sportliche Betätigung, in Form der Bereitstellung von niedrigschwelligen Angeboten, geschaffen wird.

Die Erwartungen an den Sport lassen sich auf zwei Ebenen differenzieren, die in den beschriebenen Programmen deutlich werden. Diese Ebenen sind eine Integration in Sport und eine Integration durch Sport. Integration in Sport umfasst dabei eine Art Binnenintegration und meint den Zugang zum Sportverein und auch den sozialen Einbezug in selbigen (Braun/Finke 2010: 19). Weiter wird auch die Erwartung der Integration durch Sport angesprochen, im Rahmen derer in den Vereinen bestimmte Kompetenzen und Dispositionen erlernt werden und dem Sport damit eine gewisse Sozialisationsleistung zugeschrieben wird. Die Integration durch Sport bezieht sich darüber hinaus auf eine Art Außenintegration, da auch davon ausgegangen wird, dass Sportvereine über ihre Angebote Individuen in die Gesellschaft integrieren, indem die im Verein erlernten Kompetenzen und auch Dispositionen auf andere Lebensbereiche übertragen werden können (Braun/Finke 2010: 19). Zunächst ist jedoch die Binnenintegration in den Verein der Außenintegration vorgelagert (Braun/Finke 2010: 20).

Die in den Programmen formulierten Erwartungen lassen sich diesen beiden Integrationsebenen zuordnen. So werden im Nationalen Integrationsplan Integrationschancen auf der sozialen, kulturellen und alltagspolitischen Ebene gesehen. Überwiegend geht es dabei um die 
Einbindung in die Vereine, aber auch um eine gewisse Sozialisationsleistung der Vereine durch die Vermittlung bestimmter Kulturtechniken wie beispielsweise Sprache oder Verhaltensmuster. Diese beiden Ebenen werden auch im Nationalen Aktionsplan Integration und im vorgestellten Programm „Modellregionen Integration“ der hessischen Landesregierung angesprochen. Der DOSB geht mit seinen Erwartungen an den Sport noch einen Schritt weiter und spricht auch deutlich die Übertragung der im Sportverein erlernten Kompetenzen auf andere gesellschaftliche Bereiche an. Darüber hinaus weist der DOSB dem Sport eine ,tragende Rolle im gesamtgesellschaftlichen Integrationsprozess““ zu (DOSB 2014a: 8).

Die hohen Erwartungen an den Sport in Bezug auf Integration werden von manchen Autorinnen und Autoren als überzogen bewertet (Vgl. Braun 2006, Nobis/Fussan 2007, Burrmann/Mutz/Zender 2011, Mutz 2018). Dabei wird vor allem die Integration durch Sport kritisiert, während sich für eine Integration in Sport mehr Anhaltspunkte finden lassen. Im Folgenden findet eine Diskussion der Integrationserwartungen an den Sport statt. Dazu wird zunächst auf die Ebene der Integration in den Sport eingegangen, bevor eine Diskussion der Integration durch den Sport folgt.

Im Rahmen der Integration in den Sport stellt sich die Frage nach der Einbindung von Personen in den Sportverein. Vergleicht man die Aktivitätsgrade von Personen in unterschiedlichen Freiwilligenorganisationen, wird deutlich, dass die meisten Personen im Sportvereinswesen organisiert sind (Braun/Nobis 2011: 13). Dabei beteiligen sich vor allem Heranwachsende in Sportvereinen (Nobis/Fussan 2007: 265).

Im Jahr 2018 gab es in Deutschland insgesamt 89.121 Sportvereine, deren Anzahl über die Jahre kontinuierlich abgenommen hat (vgl. Tabelle 1). Auch in Hessen hat die Anzahl der Sportvereine über die Jahre abgenommen und weist im Jahr 2017 7.629 Vereine auf ${ }^{5}$

Tabelle 1: Anzahl der Sportvereine in Deutschland in den Jahren 2014 bis 2018

\begin{tabular}{|l|l|l|l|l|}
\hline $\mathbf{2 0 1 4}$ & $\mathbf{2 0 1 5}$ & $\mathbf{2 0 1 6}$ & $\mathbf{2 0 1 7}$ & $\mathbf{2 0 1 8}$ \\
\hline 90.802 & 90.240 & 90.025 & 89.594 & 89.121 \\
\hline
\end{tabular}

Quelle: Eigene Darstellung nach Statistischen Jahrbüchern 2015-2019.

Im Gegensatz dazu zeigt die nachfolgende Tabelle 2, dass die Zahl der Mitglieder von 2014 bis 2018 zugenommen hat. Diese beträgt im Jahr 2018 in Deutschland 23.911.174 Mitglieder.

\footnotetext{
${ }^{5}$ Siehe Anhang
} 
Gleiches zeigt sich auch in Hessen. Hier stieg die Mitgliederzahl von 2.039.871 im Jahr 2014 auf 2.093.052 im Jahr $2018 \mathrm{an}^{6}$.

Tabelle 2: Anzahl der Mitglieder in Sportvereinen in Deutschland in den Jahren 2014 bis 2018

\begin{tabular}{|l|l|l|l|l|}
\hline $\mathbf{2 0 1 4}$ & $\mathbf{2 0 1 5}$ & $\mathbf{2 0 1 6}$ & $\mathbf{2 0 1 7}$ & $\mathbf{2 0 1 8}$ \\
\hline 23.691 .030 & 23.711 .811 & 23.814 .122 & 23.786 .959 & 23.911 .174 \\
\hline
\end{tabular}

Quelle: Eigene Darstellung nach Statistischen Jahrbüchern 2015-2019.

Angaben, wie viele der Mitglieder einen Migrationshintergrund besitzen, existieren jedoch nicht, da dieses Merkmal von den Sportvereinen nicht erfasst wird (Breuer/ Feiler 2017a: 175). Abhilfe schaffen hier lediglich Schätzungen zum Anteil der Personen mit Migrationshintergrund der Vereine sowie Sekundäranalysen zum Organisationsgrad von Personen mit Migrationshintergrund in Sportvereinen.

Die nachfolgende Abbildung 1 zeigt die Ergebnisse des Sportentwicklungsberichts 2015/2016 zu den Schätzungen der Vereine über den Anteil der Mitglieder mit Migrationshintergrund. Die Daten stammen aus einer Online-Umfrage, an der überwiegend Vorstandsmitglieder von Vereinen teilnahmen (Breuer/Feiler 2017b: 784). Es zeigt sich, dass ein Viertel der Vereine einen Anteil von Personen mit Migrationshintergrund von $0 \%$ angibt. $53 \%$ der Vereine geben lediglich einen Anteil von 1 bis $10 \%$ an. $15 \%$ der Vereine sehen den Anteil von Personen mit Migrationshintergrund bei 11 bis $25 \%, 4 \%$ der Vereine bei 26 bis $50 \%, 1 \%$ der Vereine bei 51 bis $75 \%$ und ebenfalls $1 \%$ der Vereine bei mehr als $75 \%$. 
Abbildung 1: Geschätzte Anteile von Personen mit Migrationshintergrund in Sportvereinen

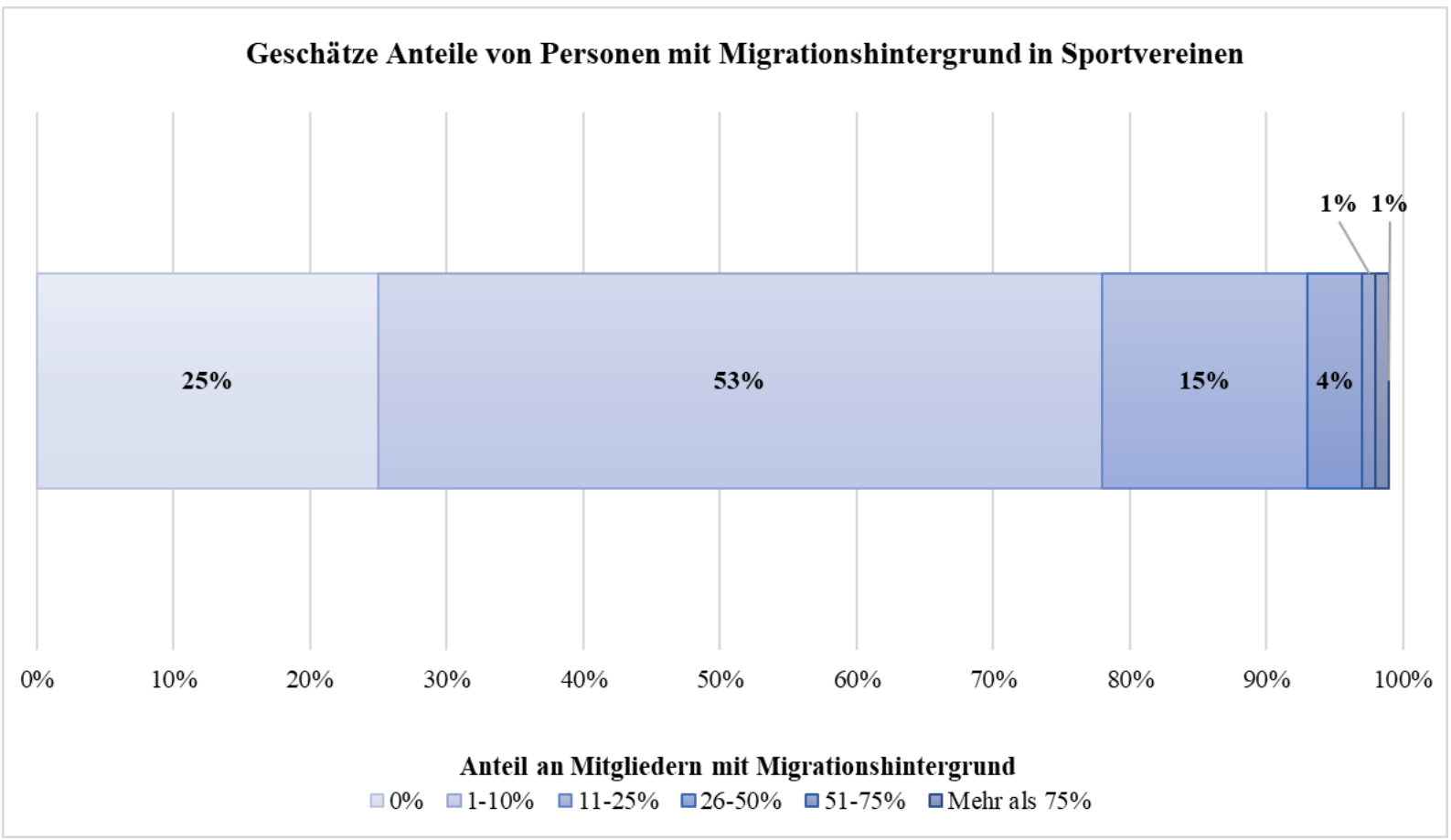

Quelle: Eigene Darstellung nach Breuer/Feiler 2017a.

Die Problematik bei diesen Schätzungen ist, dass es sich dabei um subjektive Einschätzungen derjenigen Personen handelt, die den Fragebogen ausfüllen. Darüber hinaus stellt sich auch die Frage, wie die Befragten einen Migrationshintergrund definieren. Probleme ergeben sich außerdem durch mögliche Schwankungen im Längsschnitt, da die Verantwortlichen im Verein, die den Fragebogen ausfüllen, wechseln könnten (Breuer/Feiler 2015: 234). Somit sind diese Angaben eher als grobe Orientierungsgrößen zu sehen.

Neben diesen Schätzungen soll daher noch ein Blick auf Untersuchungen zum Organisationsgrad von Personen mit Migrationshintergrund in Sportvereinen geworfen werden. Der Organisationsgrad misst dabei den prozentualen Anteil im Verein organisierter Personen innerhalb einer bestimmten Bevölkerungsgruppe (Mutz 2013: 9).

Mutz (2013) zieht im Rahmen seiner Expertise für den DOSB den Datensatz des Projektes „Aufwachsen in Deutschland: Alltagswelten“, kurz AID:A heran. Innerhalb seiner Analyse betrachtet er drei Altersgruppen: Kinder von 2 bis 12 Jahren, Jugendliche von 13 bis 17 Jahren und Erwachsene von 18 bis 55 Jahren. Es zeigt sich, dass Sportvereine vor allem für Kinder große Bindungskräfte aufweisen und in dieser Altersgruppe $71 \%$ der Kinder ohne Migrationshintergrund und $55 \%$ der Kinder mit Migrationshintergrund in Vereinen aktiv sind (Mutz 2013: 12). Im Jugendalter nähern sich die Organisationsgrade der beiden Gruppen an. Hier sind $65 \%$ der Jugendlichen ohne Migrationshintergrund und $54 \%$ der Jugendlichen mit 
Migrationshintergrund Mitglied in einem Sportverein (Mutz 2013: 15/16). Mit dem Eintritt ins Erwachsenenalter nimmt der Organisationsgrad in beiden Gruppen jedoch stark ab und es sind nur noch $33 \%$ der Personen mit Migrationshintergrund und $28 \%$ der Personen ohne Migrationshintergrund Sportvereinsmitglieder (Mutz 2013: 17/18). Dies hängt vermutlich mit den Anforderungen in Schule und Beruf zusammen, die dazu führen, dass selbst organisierte Sportmöglichkeiten favorisiert werden (Mutz/Burrmann 2015: 74).

In einer weiteren Arbeit stellen Mutz und Burrmann (2015) Sekundäranalysen zum Organisationsgrad unterschiedlicher Datensätzen gegenüber. Es zeigt sich, dass in drei großen Jugendstudien (AID:A, SPRINT, Nationale Ergänzungsstudie zu PISA) der Organisationsgrad von Jugendlichen mit und ohne Migrationshintergrund keine großen Unterschiede aufweist. Jedoch zeigen sich erhebliche Unterschiede innerhalb der Gruppe der Jugendlichen mit Migrationshintergrund, wenn man den Faktor Geschlecht betrachtet. So sind nach dem Datensatz AID:A 60 \% der männlichen Jugendlichen mit Migrationshintergrund Mitglied in einem Sportverein. Bei den weiblichen Jugendlichen mit Migrationshintergrund beträgt dieser Anteil lediglich $31 \%$. Ähnliche Ergebnisse zeigen sich auch bei der Nationalen Ergänzungsstudie zu PISA und SPRINT. Bei den Jugendlichen ohne Migrationshintergrund ergeben sich, bezogen auf das Geschlecht, im Rahmen der Auswertung der unterschiedlichen Datensätze, nur Unterschiede von etwa 10 Prozentpunkten (Mutz/Burrmann 2015: 71).

Die vorangegangenen Ausführungen zeigen, dass es keine validen Zahlen zur Anzahl von Personen mit Migrationshintergrund in deutschen Sportvereinen gibt. Dennoch scheinen Sportvereine starke Bindungskräfte für Personen mit und ohne Migrationshintergrund aufzuweisen. Der Rückgriff auf Schätzungen der Vereine und Sekundäranalysen zum Organisationsgrad zeigen, dass sich der Organisationsgrad zwischen Personen mit und ohne Migrationshintergrund nicht stark unterscheidet. Jedoch sind gerade Mädchen und Frauen mit Migrationshintergrund in Vereinen deutlich weniger organisiert. Für die vorliegende Arbeit bedeutet dies, dass die subjektiven Sichtweisen dieser Gruppe unterrepräsentiert sein könnten.

Die Ausführungen zeigen darüber hinaus, dass viele Personen, auch mit Migrationshintergrund, in Sportvereinen aktiv sind. Eine Integration in die Sportvereine, in Form des Zugangs zum Verein, findet also statt. Fraglich ist hierbei jedoch, ob auch eine soziale Integration in die Wahl-Gemeinschaft des Sportvereins stattfindet. Im wissenschaftlichen Diskurs wird angeführt, dass Interaktionen durch eine regelmäßige und auch langfristige Teilnahme in einem Sportverein begünstigt werden können und die Mitgliedschaft so zum Aufbau sozialer Kontakte und Bindungen führen kann (Nobis/Fussan 2007: 266). Für diese Interaktionen bietet der 
Sportverein Begegnungsmöglichkeiten und Aktivitäten mit Gleichaltrigen (Becker/Häring 2012: 261).

Fussan (2006) zeigt im Rahmen einer Untersuchung, dass jugendliche Sportvereinsmitglieder über ein größeres Peer-Netzwerk verfügen als Nicht-Sportvereinsmitglieder. Darüber hinaus sind die in Vereinen organisierten Jugendlichen zentraler in ihren Peer-Netzwerken organisiert und haben häufiger Kontakt zu ihren Peers als Jugendliche, die keinem Verein angehören (Fussan 2006: 397/398). Jedoch konnte mit Hilfe der Daten nicht ermittelt werden, wie diese Peers aufgebaut sind. Ob sie also nur aus anderen Sportvereinsmitgliedern bestehen oder andere Vernetzungen der Jugendlichen vorhanden sind, bleibt damit unklar. Darüber hinaus merkt Fussan an, dass Selektionseffekte vorliegen könnten und sich gerade Jugendliche in Vereinen organisieren, die bereits über größere Peers verfügen und weniger Probleme damit haben, neue Kontakte aufzubauen (Fussan 2006: 399/400).

Becker und Häring (2012) weisen unter Kontrolle soziodemographischer Faktoren (verschiedene Persönlichkeitseigenschaften und Merkmale zur Lebenssituation) nach, dass sportlich aktive Personen einen signifikant größeren Freundes- und Bekanntenkreis besitzen, sich häufiger mit diesen Personen treffen und sich in mehr Kontexten aufhalten als Nichtsportlerinnen und -sportler (Becker/Häring 2012: 266). Dabei zeigen sich in der Gruppe der Sportlerinnen und Sportler nur geringe Unterschiede bei Berücksichtigung von Organisationsformen (vereinsorganisierter Sport oder ohne Vereinszugehörigkeit) und Sportart (Mannschaftssport oder Individualsport). „Der regelmäßig wiederkehrende Kontakt mit denselben Menschen in Sportkontexten scheint somit die stärkste Erklärungskraft für die soziale Integration der Befragten zu besitzen - weniger die Art oder Organisationsform des Sports. Ein interessanter Befund ist hierbei, dass selbst unter Kontrolle von Indikatoren, die gemäß dem Stand der Forschung ebenfalls einen Einfluss auf die soziale Integration haben, der positive Effekt der Sportaktivität erhalten bleibt. Gerade hinsichtlich der Befunde zur Größe des Freundeskreises und der Häufigkeit des Kontakts mit Freunden und Bekannten kann abgeleitet werden, dass es sich bei Sportaktivitäten um Kontexte handelt, die es - auch unter Kontrolle von z. B. dem Alter oder der Erwerbstätigkeit - erleichtern, in regelmäßigen sozialen Kontakt zu bleiben“ (Becker/Häring 2012: 268).

Im Rahmen der Integration durch Sport stellen sich zwei Fragen. Diese sind zum einen die Frage, ob Sportvereine eine Art Sozialisationsleistung erbringen, indem sie Werte, Normen oder Haltungen vermitteln. Zum anderen stellt sich die Frage des Transfers von erlernten Kompetenzen und Dispositionen auf andere Lebensbereiche. 
Eine Sozialisationsleistung der Sportvereine, in Form der Vermittlung von Normen, Werten und Haltungen, ist nach Mutz empirisch nur punktuell belegt und es bleibt unklar, ob Sport auch über seinen Nahbereich hinaus, also in die Gesellschaft hinein, Integrationsprozesse anregt (Mutz 2018: 175/176). Innerhalb seiner Untersuchung zeigt sich kein Effekt des Sportengagements auf erwartete spätere Bildungsabschlüsse und Berufspositionen und auch keine Zusammenhänge mit der Persönlichkeitsentwicklung und der Sozialintegration. Lediglich im Rahmen der Einbindung in die Gruppe der Gleichaltrigen zeigen sich kleine Effekte (Mutz 2018: 306/307). Dazu schlussfolgert Mutz: „Wer eine Integration durch Sport als „Nebenprodukt“ des ganz normalen Sporttreibens in Sportvereinen und Sportarbeitsgemeinschaften erwartet, überschätzt die Leistungen offensichtlich, die der organisierte Sport erbringen kann“ (Mutz 2018: 307).

Burrmann, Mutz und Zender (2011) stellen die untergeordnete Rolle des Sports im Vergleich zu anderen Sozialisationsinstanzen, wie Familie, Schule und Freundeskreis, heraus. Nach Meinung der Autorinnen und des Autors ist eine Integration in die Gesellschaft nur in Einzelfällen stark durch die Sportvereinsmitgliedschaft geprägt und muss im Verein aktiv durch Maßnahmen unterstützt werden. Denn automatisch stellen sich diese Integrationsvorteile nicht ein. Zwar lassen sich Integrationseffekte teilweise nachweisen, diese besitzen jedoch nur einen sehr geringen Umfang und zeigen sich auch nur in wenigen Bereichen, wie beispielsweise der Selbstwirksamkeit der Personen (Burrmann/Mutz/Zender 2011: 262). Auch wenn die Sportvereine bestimmte Bindungskräfte für Personen mit und ohne Migrationshintergrund aufweisen, kann daraus nicht geschlussfolgert werden, dass gleichzeitig auch eine Integration in die Gesellschaft stattfindet (Burrmann/Mutz/Zender 2011: 262).

Braun und Finke (2010) beschreiben die Außenintegration als ,nicht weiter elaborierte Transferannahme“ (Braun/Finke 2010: 19). Und auch Nobis und Fussan sind der Meinung, dass die Erwartungen an den Sport heruntergeschraubt werden sollten, da Sport Defizite aus anderen Bereichen, wie beispielsweise der Bildungsteilhabe und dem Spracherwerb, nicht komplett ausgleichen kann (Nobis/Fussan 2007: 266). Mutz (2018) stellt dazu fest, dass dem Sport, bei Zutreffen der in Programmen formulierten Erwartungen, eine immense Bedeutung für das Gemeinwohl zukommen würde. Ob diese Erwartungen jedoch zutreffen, ist eher fraglich (Mutz 2018: 174). 


\subsubsection{Schlussfolgerungen für die eigene Arbeit}

Die hohen Erwartungen an den Sport im Rahmen der Integration werden von vielen Autorinnen und Autoren skeptisch gesehen. Braun (2006) schreibt dazu: „Allerdings haben diese Thesen in erster Linie den Status von normativ gefärbten Annahmen mit Plausibilitätsanspruch, da es sowohl an komplexeren theoretischen Argumentationszusammenhängen als auch an empirischen Daten zu der Frage mangelt, wie sich der Einbezug in die Wahl-Gemeinschaft vollziehen könnte und über welche Mechanismen die dort ggf. erworbenen sozialen und politischen Orientierungen in andere gesellschaftliche Kontexte vermittelt werden können“ (Braun 2006: 449). Weiter führt Braun aus, dass die Daten und ermittelten Zusammenhänge keine Informationen darüber liefern, was sich innerhalb von Vereinen tatsächlich abspielt. In diesem Zusammenhang spricht er von Sportvereinen als eine Art „blackbox“. Somit lassen sich keine Aussagen über in den Vereinen stattfindende Sozialisationsprozesse treffen (Braun 2006: 4504).

Die vorangegangenen Ausführungen haben jedoch gezeigt, dass Sport gewisse Bindungskräfte für Personen mit und ohne Migrationshintergrund aufweist und auch Anhaltspunkte für eine verbesserte Sozialintegration von sportlich aktiven Personen liegen vor. Dabei ist jedoch nicht klar, ob die bessere Sozialintegration durch die Sportaktivität beeinflusst wird oder Selektionseffekte der Teilnehmenden vorliegen. Die Betonung dieser sozialintegrativen Wirkung in Bezug auf die Integration in Sport lässt vermuten, dass bei den Befragten gerade die Sozialintegration in den subjektiven Sichtweisen auf Integration sichtbar werden wird. Im Gegensatz zur Integration in Sport ist die Integration durch Sport deutlich umstrittener und weist kaum empirische Anhaltspunkte auf.

Dennoch machen diese Integrationserwartungen an den Sport im öffentlichen Diskurs, sowie Präsenz des Themas Sport in der Integrationspolitik, Sportorganisationen zu einem interessanten Untersuchungsgegenstand für subjektive Sichtweisen auf Integration. Zum einen kann davon ausgegangen werden, dass gerade auf Ebene der Vorstände und anderer Funktionärinnen und Funktionäre eine gewisse Sensibilisierung für das Thema Integration und dessen Ausgestaltung vorliegt. Zum anderen bietet gerade der Sport als favorisierte Organisation in der Freizeitgestaltung und durch seine Bindungskräfte für Personen mit und ohne Migrationshintergrund Möglichkeiten des Zusammentreffens und der Interaktion zwischen Personen unterschiedlicher Kulturen. Dadurch lassen sich auch Vergleiche zwischen Personen mit und ohne Migrationshintergrund anstellen, die im Setting des Sportvereins zusammenkommen. 


\subsection{Subjektive Sichtweisen auf Integration}

Im Rahmen dieses Kapitels werden zunächst Studien dargestellt, die sich mit der subjektiven Sichtweise auf Integration beschäftigen. Die dargestellten Arbeiten beziehen sich nicht nur auf Sportorganisationen als Untersuchungsgegenstand, sondern auch auf die Bereiche Soziale Arbeit und Schule sowie Sichtweisen auf Integration im Allgemeinen. In einem weiteren Unterkapitel erfolgt dann eine Betrachtung von Arbeiten, die sich mit dem Zusammenhang zwischen subjektiven Sichtweisen auf Integration und sozialstrukturellen Merkmalen auseinandersetzen, da Zusammenhänge mit sozialstrukturellen Merkmalen auch im Rahmen der vorliegenden Arbeit überprüft werden sollen. Den Abschluss bildet eine Einordnung des Forschungsstandes zu subjektiven Sichtweisen mit Schlussfolgerungen für die vorliegende Arbeit, welche auch der Vorbereitung der Formulierung von Hypothesen in Kapitel 4.3 dienen.

\subsubsection{Forschungsarbeiten zu subjektiven Sichtweisen auf Integration}

In der bisherigen Forschung fanden subjektive Sichtweisen auf Integration, in Form des Verständnisses von Integration und der Favorisierung bestimmter Ausgänge von Integration, wenig Beachtung. Arbeiten zu dieser Thematik umfassen zum einen Arbeiten, die sich mit der Einstellung zu Ausländerinnen und Ausländern, Integration und Akkulturation beschäftigen (Van Dick et al. 1997; Zick/Preuß 2016; Blohm/Wasmer 2018). Zum anderen existieren Arbeiten, die vor allem die Unterstützung von Integrationskonzepten untersuchen (Strahle/Baur 2009, Baur/Mutz 2009; Hachfeld et al. 2011, 2012, 2015; Civitillo et al. 2017, 2019) sowie Arbeiten zum konkreten Integrationsverständnis (Lutter 2011; Hanhart 2020; Schramkowski 2007, 2010). Insgesamt zeigt sich auch, dass diese Arbeiten unterschiedliche Bereiche, wie Sportvereinsforschung, soziale Arbeit und Schulforschung, umfassen.

In ihrer Arbeit zur Einstellung gegenüber Ausländerinnen und Ausländern ziehen Blohm und Wasmer (2018) die Allgemeine Bevölkerungsumfrage der Sozialwissenschaften (ALLBUS) heran. Im ALLBUS wird die Einstellungen gegenüber Ausländerinnen und Ausländern regelmäßig anhand folgender Items erhoben:

- Die in Deutschland lebenden Ausländer sollten ihren Lebensstil (ein bisschen) besser an den der Deutschen anpassen.

- Wenn Arbeitsplätze knapp werden, sollte man die in Deutschland lebenden Ausländer wieder in ihre Heimat zurückschicken. 
- Man sollte den in Deutschland lebenden Ausländern jede politische Betätigung in Deutschland untersagen.

- Die in Deutschland lebenden Ausländer sollten sich ihre Ehepartner unter ihren eigenen Landsleuten auswählen (Blohm/Wasmer 2018: 403).

Insbesondere das erste Item stellt dabei die Einstellung zu einem Ausgang von Integration, nämlich der Assimilation in Form von Anpassung, dar. Diesem Item wurde im Jahr 2016 auch am meisten zugestimmt. Insgesamt über $70 \%$ der Befragten in Westdeutschland und über $80 \%$ in Ostdeutschland stimmten dieser Aussage zu. Dabei stimmten Personen mit (Fach-) Hochschulreife (62\%) diesem Item seltener zu als Personen mit mittlerer Reife (74\%) oder einem Hauptschulabschluss (82\%). Die anderen Items wurden im Gegensatz dazu eher abgelehnt (Blohm/Wasmer 2018: 404). Insgesamt kann somit also festgehalten werden, dass das Konzept der Assimilation in der Bevölkerung großen Zuspruch findet und sich die Bewertung des Konzepts zwischen den Bildungsgruppen, auf deskriptiver Ebene, unterscheidet.

Zick und Preuß (2016) untersuchten in ihrer Arbeit Einstellungen der Bevölkerung zu Integration. Die Umfragen erfolgten zum Jahreswechsel 2013/2014 und 2015/2016. Im Rahmen der ersten Befragung wurden 2.006 repräsentativ ausgewählten Personen ab 18 Jahren befragt. Die zweite Umfrage umfasste 1.300 repräsentativ ausgewählten Personen sowie eine zusätzliche Stichprobe von 205 Personen mit Migrationshintergrund ab 16 Jahren. Insgesamt umfasste die Arbeit mehrere Fragestellungen zu den Bereichen Willkommenskultur, Vorrechte, Kriterien der Zugehörigkeit, Vorstellungen zum Zusammenleben sowie Einstellungen zu Geflüchteten (Zick Preuß 2016: 2). Für die vorliegende Arbeit von Interesse sind insbesondere die Ergebnisse $\mathrm{zu}$ den Kriterien der Zugehörigkeit sowie die Vorstellungen zum Zusammenleben. In Bezug auf die Kriterien der Zugehörigkeit zeigte sich in beiden Befragungen, dass vor allem die Achtung politischer Institutionen und Gesetze, die Deutsche Sprache, das Gefühl sich in Deutschland Zuhause zu fühlen und die Erwerbstätigkeit hohe Relevanz besitzen. Bei Personen ohne Migrationshintergrund nimmt dabei die Achtung politischer Institutionen und Gesetzte die wichtigste Rolle ein (82\% Zustimmung), während bei Personen mit Migrationshintergrund die Sprache die höchste Zustimmung erfährt (90\%) (Zick/Preuß 2016: 11-13).

Darüber hinaus wurde im Rahmen der Vorstellungen zum Zusammenleben durch die Zustimmung zu bzw. Ablehnung von zwei Fragen die Einstellung zu den Konzepten Integration 


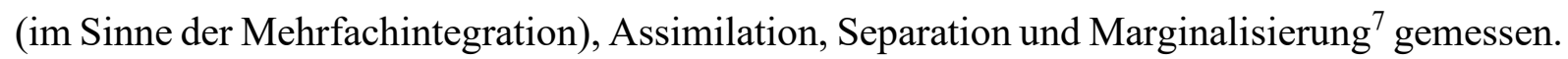
Es zeigte sich, dass das Konzept der Integration insgesamt die höchste Zustimmung aufwies, auch wenn diese in der zweiten Umfrage geringer ausfiel als in der ersten (Zick/Preuß 2016: 16). Im Gegensatz dazu stieg die Zustimmung zu den Konzepten Assimilation und Separation leicht an (Zick/Preuß 2016: 26). Dabei zeigte sich in der zweiten Umfrage bei Personen mit Migrationshintergrund eine höhere Zustimmung zur Integration, Separation und Marginalisierung als bei Personen ohne Migrationshintergrund. Jedoch zeigten sich nur in Bezug auf die Integration signifikante Unterschiede (Zick/Preuß 2016: 15/16).

Van Dick et al. konstruierten 1997 auf Grundlage des Modells zur Akkulturation nach Berry et al. (1977) einen Fragebogen zur Messung der Einstellung von Menschen im deutschen Sprachraum zur Akkulturation (van Dick 1997: 1). Dabei wurden die Dimensionen Assimilation, Integration und Segregation anhand von 13 Items erfasst. Insgesamt stellte sich das konstruierte Messinstrument als zufriedenstellend reliabel und valide heraus. Es zeigte sich jedoch auch, dass die Items lediglich auf einen Faktor laden. Dies wird in der Arbeit damit, dass Personen, die die Integrationsitems befürworten, die Items zu Assimilation und Segregation ablehnen. Im Gegensatz dazu befürworten Personen, die die Integrationsitems ablehnen, die Items zu Assimilation und Segregation (van Dick 1997: 4).

In Bezug auf favorisierte Integrationskonzepte finden sich Arbeiten in der Schul- und Sportvereinsforschung.

Im Rahmen der Untersuchung von migrationsbezogenen Überzeugungen von Lehrkräften und deren Zusammenhänge mit professionellen Kompetenzen sowie dem Unterrichtsverhalten entwickelten Hachfeld et al. (2011) die „Teacher Cultural Beliefs Scale“ (TCBS), auf die auch weitere Arbeiten aufbauen. Diese Skala misst zum einen multikulturelle und zum anderen egalitäre Überzeugungen. Multikulturelle Überzeugungen ${ }^{8}$ umfassen dabei eine allgemeine Wertschätzung kultureller Unterschiede, welche auch in der Unterrichtpraxis berücksichtigt werden sollten. Egalitäre Überzeugungen betonen hingegen die Gleichbehandlung aller Schülerinnen und Schüler, unabhängig von ihrem kulturellen Hintergrund (Hachfeld et al. 2011: 993/994). Anhand der entworfenen Skala führten Hachfeld et al. in ihrem Artikel „Assessing teachers' multicultural and egalitarian beliefs: The Teacher Cultural Beliefs Scale“ zwei Untersuchungen durch, um zum einen die zwei-Faktor-Struktur der Skala zu bestätigen und zum anderen Zusammenhänge mit der Motivation für vorurteilsfreie Reaktionen sowie

\footnotetext{
${ }^{7}$ Zur Erläuterung dieser Konzepte siehe Kapitel 4.

${ }^{8}$ Zur weiteren Erläuterung dieses Konzepts siehe Kapitel 4.
} 
Ansichten zu Akkulturation, Vorurteilen und Autoritarismus zu analysieren (Hachfeld et al. 2011: 986). Die erste Studie umfasste eine Stichprobe von 433 angehenden Lehrenden in der Phase des zweijährigen Vorbereitungsdienstes aus der Referendariatsstudie (COACTIV-R) (Hachfeld et al. 2011: 989). Die zweite Stichprobe bezog sich auf 340 Studierende im Bereich Lehramt oder Erziehungswissenschaften, die an einer deutschen Universität befragt wurden (Hachfeld et al. 2011: 992). Anhand der ersten Studie konnte die zwei-Faktor-Struktur der Skala bestätigt werden. Es zeigten sich jedoch auch Unterschiede in der Bewertung der beiden Überzeugungen je nach den vorhandenen Erfahrungen mit kultureller Vielfalt. So befürworteten angehende Lehrende an Berufsschulen den Multikulturalismus stärker als angehende Lehrende an Hochschulen. Generell weisen Berufsschulen einen höheren Anteil an Schülerinnen und Schüler mit Migrationshintergrund als Hochschulen auf, woraus sich für die Lehrenden an Berufsschulen ein vermehrter Umgang mit kultureller Vielfalt ergibt. Darüber hinaus befürworteten auch Befragte mit Migrationshintergrund den Multikulturalismus stärker als diejenigen ohne Migrationshintergrund. Innerhalb der zweiten Studie zeigte sich, dass Multikulturalismus und Egalitarismus gleichermaßen mit der Motivation zusammenhängen, vorurteilsvolles Verhalten $\mathrm{zu}$ kontrollieren, aber nicht mit dem Widerstand gegen vorurteilsvolle Aussagen. So zeigte sich ein negativer Zusammenhang zwischen Multikulturalismus und Vorurteilen, während Egalitarismus keinen Zusammenhang aufwies. Somit führen multikulturelle Überzeugungen eher $\mathrm{zu}$ einer generellen Ablehnung von Vorurteilen. Egalitäre Überzeugungen hängen im Gegensatz dazu nur mit dem Willen zusammen Vorurteile zu kontrollieren. Außerdem zeigten sich positive Zusammenhänge der Multikulturalismus und Egalitarismus mit den Skalen zu Pluralismus und Akkulturation. In Bezug auf den Multikultarlismus fielen diese Zusammenhänge jedoch stärker aus. In Bezug auf die Skala zu Autoritarismus zeigte sich lediglich ein negativer Zusammenhang mit dem Multikulturalismus. Dies zeigt, dass eine egalitäre Perspektive im Gegensatz zu multikulturellen Überzeugungen nicht im Widerspruch zum Autoritarismus steht (Hachfeld et al. 2011: 994).

Aufbauend auf diesen Ergebnissen beschäftigten sich Hachfeld et al. in ihrem Artikel „Multikulturelle Überzeugungen“ (2012) mit der Rolle des Migrationshintergrundes sowie multikultureller Überzeugungen für das Unterrichten von Kindern mit Migrationshintergrund. Im Rahmen dessen sollte untersucht werden, ob sich angehende Lehrende mit und ohne Migrationshintergrund in ihren professionellen Kompetenzen unterscheiden und, ob multikulturelle Überzeugungen eine vermittelnde Rolle zwischen Migrationshintergrund und professioneller Kompetenz aufweisen (Hachfeld et al. 2012: 108). Datenbasis für die Analysen 
war erneut die Referendariatsstudie (COACTIV-R). Die Stichprobe umfasste 433 angehende Lehrende, von denen 60 (14,2 \%) einen Migrationshintergrund besaßen (Hachfeld et al. 2012: 109). Die Mittelwertvergleiche zeigten, dass angehende Lehrende mit Migrationshintergrund höheren Enthusiasmus und höhere Selbstwirksamkeitserfahrungen für das Unterrichten von Schülern mit Migrationshintergrund berichteten als Lehrende ohne Migrationshintergrund. Darüber hinaus wiesen Lehrende mit Migrationshintergrund auch stärkere multikulturelle Überzeugungen auf. Die im Anschluss berechneten Strukturgleichungsmodelle ohne Berücksichtigung der multikulturellen Überzeugungen (mit den Kontrollvariablen Alter, sozioökonomischer Status und Gesamtnote des ersten Staatsexamens) zeigten jedoch, dass die angehende Lehrende mit Migrationshintergrund nur in Bezug auf ihren Enthusiasmus für das Unterrichten von Schülerinnen und Schülern mit Migrationshintergrund von angehenden Lehrenden ohne Migrationshintergrund unterschieden. Unter Berücksichtigung der multikulturellen Überzeugungen reduzierten sich die Zusammenhänge zwischen Migrationshintergrund und den untersuchten Kompetenzen jedoch signifikant oder waren nicht mehr vorhanden, was für eine Mediation bzw. vermittelnde Rolle von multikulturellen Überzeugungen spricht. Zusammenfassend wird im Rahmen der Arbeit festgehalten, dass ein vorhandener Migrationshintergrund vielleicht weniger entscheidend für professionelle Kompetenzen im Kontext kultureller Heterogenität im schulischen Unterricht ist als multikulturelle Überzeugungen (Hachfeld et al. 2012: 114/115). „Der Vorsprung von Lehramtsanwärter(inne)n mit Migrationshintergrund hinsichtlich ihres Enthusiasmus für das Unterrichten von Schülern mit Migrationshintergrund könnte stattdessen zu einem gewissen Teil mit ihren stärker ausgeprägten multikulturellen Überzeugungen zusammenhängen“ (Hachfeld et al. 2012: 115).

In einer weiteren Arbeit betrachteten Hachfeld et al. (2015) Zusammenhänge zwischen multikulturellen sowie egalitären Überzeugungen und beruflichen Kompetenzen von angehenden Lehrenden für das Unterrichten von Schülerinnen und Schülern mit Migrationshintergrund. Die Stichprobe umfasste erneut 433 Befragte im Rahmen der Referendariatsstudie (COACTIV-R). Die Pfadmodellanalysen zeigten, dass Befragte mit multikulturellen Überzeugungen höhere Motivationsorientierungen (Selbstwirksamkeit, Begeisterung für den Unterricht, integrativere Karrieremotive), eine geringere Übereinstimmung mit negativen Stereotypen sowie eine höhere Bereitschaft besaßen, ihre Lehre vor dem Hintergrund steigender kultureller Heterogenität in der Schülerschaft anzupassen. Bei egalitären Überzeugungen zeigte sich hingegen ein negativer Zusammenhang mit der Bereitschaft den Unterricht an die Schülerinnen und Schüler anzupassen. Für die 
restlichen Konstrukte konnten darüber hinaus keine Zusammenhänge mit egalitären Überzeugungen festgestellt werden (Hachfeld et al. 2012: 44).

Auch in der Arbeit von Civitillo et al. (2017) spielt der Kontext Schule eine Rolle, um zu untersuchen, welche Arten von deskriptiven Normen in Bezug auf kulturelle Vielfalt (Egalitarismus oder kultureller Pluralismus) von Lehrenden sowie von Schülerinnen und Schülern mit und ohne Migrationshintergrund in Schulen wahrgenommen werden und wie diese Wahrnehmungen mit Praktiken und Artefakten in der Schulumgebung zusammenhängen (Civitillo et al. 2017: 3). Die Daten umfassten Angaben von 207 Lehrenden und 1644 Schülerinnen und Schülern der sechsten Klassen von 22 Schulen in Deutschland. Darüber hinaus wurden 2995 Fotos der Schulumgebung der befragten Klassen ausgewertet (Civitillo et al. 2017: 4). In allen untersuchten Schulen nahmen Lehrende sowie Schülerinnern und Schüler eher Normen wahr, die auf Egalitarismus ausgerichtet waren. Anhand einer multivariaten Varianzanalyse konnten zwischen den Schülerinnen und Schülern nur in Bezug auf die Subskala „Gleichbehandlung“ ein signifikanter Unterschied zwischen Schülerinnen und Schülern mit und ohne Migrationshintergrund nachgewiesen werden. Dabei bewerteten Schülerinnen und Schüler ohne Migrationshintergrund diese Skala höher als Schülerinnen und Schüler mit Migrationshintergrund. Darüber hinaus wurden bivariate Korrelationen zwischen den Wahrnehmungen der Lehrenden und der Schülerinnen und Schüler untersucht. Diese zeigten lediglich signifikante Korrelationen zwischen den Gleichbehandlungswahrnehmungen der Lehrenden und der Schülerinnen und Schüler ohne Migrationshintergrund (Civitillo et al. 2017: 6). In Bezug auf die zweite untersuchte Fragestellung zeigte sich, anhand eines Vergleichs von sechs Schulen, dass in Schulen, in denen Lehrende und Schülerinnen und Schüler wenige egalitäre oder pluralistische Normen wahrnahmen, nur selten Artefakte, die kulturelle Diversität thematisieren, vorhanden waren. In diesen Schulen konnte die Umgebung eher einem dritten Ansatz zugeordnet werden, den die Autorinnen und Autoren als „endorsement of majority culture“ bezeichnen. Im Gegensatz dazu waren in Schulen, in denen Lehrende und Schülerinnen und Schüler egalitäre und pluralistische Normen wahrnahmen, auch entsprechende Artefakte vorhanden (Civitillo et al. 2017: 10). Insgesamt wird im Rahmen der Arbeit festgehalten, dass deskriptive Normen, Praktiken und Artefakte, die im Zusammenhang mit kultureller Vielfalt stehen, miteinander verbunden sind (Civitillo et al. 2017: 12).

In einer weiteren Studie untersuchten Civitillo et al. (2019) die Beziehungen zwischen kultursensiblem Unterricht, Überzeugungen zu kultureller Vielfalt der Lehrenden und 
Selbstreflexion in Bezug auf den eigenen Unterricht. Dazu wurden Fallstudien mit vier Lehrenden mit Hilfe von Videobeobachtungen und anschließenden Interviews durchgeführt. Es zeigte sich eine hohe Kongruenz zwischen kultursensiblem Unterricht und Überzeugungen zu kultureller Vielfalt. Darüber hinaus wiesen Lehrende, die mit einer höheren kulturellen Sensibilität auch eine höhere Selbstreflexion in Bezug auf ihren eigenen Unterricht auf (Vgl. Civitillo et al. 2019).

In der Sportvereinsforschung beschäftigen sich Baur et al., vor dem Hintergrund der Evaluation des Programms „Integration durch Sport“ (2009), unter anderem mit dem subjektiven Integrationskonzept. Dieses subjektive Integrationskonzept beschreibt Vorstellungen, Meinungen, Einschätzungen und Bewertungen in Bezug auf die Integration von Zugewanderten. Die Untersuchung dieses subjektiven Integrationskonzepts findet auf der Ebene der Landeskoordinatorinnen und -koordinatoren, Ansprechpartnerinnen und -partner sowie Übungsleiterinnen und -leiter des Programms statt. Der Fokus liegt dabei auf der Ausgestaltung und wünschenswerten Ausgängen von Integration. (Nobis/Strahle 2009: 117).

Für die Befragung der Landeskoordinatorinnen und -koordinatoren wurde eine Kombination aus leitfadengestützten, qualitativen Interviews und einer kurzen quantitativen Befragung gewählt, während die Ansprechpartnerinnen und -partner sowie die Übungsleiterinnen und leiter eine ausführlichere quantitative Befragung erhielten. Die kurze quantitative Befragung der Landeskoordinatorinnen und -koordinatoren bezog sich jedoch nicht auf das subjektive Integrationskonzept, weshalb im Folgenden nur auf die Interviews mit den Landeskoordinatorinnen und -koordinatoren sowie auf die quantitative Befragung der Ansprechpartnerinnen und -partner bzw. der Übungsleiterinnen und -leiter eingegangen wird.

Im Rahmen der Interviews mit den Landeskoordinatorinnen und -koordinatoren wurden insgesamt 17 Personen befragt. Diese sollten ihre Vorstellung zu Integration im Allgemeinem sowie in Bezug auf den Sport thematisieren. Die Fragen, die ihnen dazu gestellt wurden, lauteten „Was heißt für Sie persönlich eigentlich Integration?“ und „Was heißt für Sie Integration von Zuwanderern?“" (Strahle/Baur 2009: 131-133). Außerdem wurden den Landeskoordinatorinnen und -koordinatoren Statements $\mathrm{zu}$ Integrationskonzepten zur Bewertung vorgelegt, deren Bewertung in einem weiteren Schritt begründet werden sollte. Diese Statements umfassten die Aussagen „Zuwanderer sollten sich den Gegebenheiten der deutschen Sportorganisationen anpassen“, „Es müssen Angebote geschaffen werden, die den Bedürfnissen der Zuwanderer entsprechen“ und „Integration kann nur dann gelingen, wenn die Einheimischen auf die Zuwanderer zugehen“ (Strahle/Baur 2009: 131-133). Die Interviews 
wurden anschließend mit Hilfe einer qualitativen Inhaltsanalyse, anhand einer Kombination aus deduktivem und induktivem Vorgehen, analysiert (Rübner/Strahle/Baur 2009: 72).

Es zeigte sich zunächst, dass die Vorstellungen zu Integration im Allgemeinen und in Bezug auf den Sport nicht immer trennscharf zu unterscheiden sind. Außerdem wurde oft mit der Beantwortung der ersten Frage schon Bezug auf die Gruppe der Zugewanderten genommen, sodass sich ein expliziter Bezug des Integrationsbegriffs auf diese Gruppe vermuten lässt (Strahle/Baur 2009: 131-133). Einige Interviewpartnerinnen und -partner hatten darüber hinaus keine genaue Vorstellung von Integration, während andere sich bereits näher mit dem Thema auseinandergesetzt hatten. Hier zeigt sich somit ein Differenzierungsgrad der subjektiven Integrationsvorstellungen je nach Informationsstand (Strahle/Baur 2009: 133). In Bezug auf Elemente von Integration beschrieben einige Interviewpartnerinnen und -partner Integration als Prozess. Darüber hinaus wurde Integration auch in einigen Fällen als eine Art „Selbstläufer“ beschrieben, während andere Interviewpartnerinnen und -partner die Bedeutung integrationsfördernder Maßnahmen betonten. Somit lässt sich bezüglich der Ausgestaltung von Integration und ob dabei aktive Maßnahmen notwendig sind, kein einheitliches Bild ausmachen (Strahle/Baur 2009: 133/134).

In Bezug auf die favorisierten Integrationskonzepte der Landeskoordinatorinnen und koordinatoren zeigte sich ebenfalls kein einheitliches Verständnis, was auch wiederrum verdeutlicht, dass es nicht nur eine Sichtweise auf Integration gibt. So lassen sich innerhalb der Interviews assimilative sowie pluralistische Einstellungen erkennen. Die Anpassung an die Kultur der Aufnahmegesellschaft und somit das Konzept der Assimilation wird von 8 der 17 Landeskoordinatorinnen und -koordinatoren thematisiert. Grundlegend ist dabei unter anderem das Erlernen der Sprache, welches von den interviewten Personen am häufigsten angesprochen wird (Strahle/Baur 2009: 137/ 138). Pluralistische Einstellungen zeigen sich hingegen beispielsweise in Aussagen, die das Bestehenbleiben des Bezugs zur Herkunft beschreiben oder die Vorstellung einer möglichst gleichberechtigten gesellschaftlichen Partizipation beinhalten (Strahle/Baur 2009: 142). Darüber hinaus wird bei einigen Interviewpartnerinnen und -partnern ein beidseitiges Interesse von zu integrierenden Personen und der Aufnahmegesellschaft als Voraussetzung für Integration beschrieben. Dieses beidseitige Interesse beinhaltet nach Meinung der Interviewpartnerinnen und -partner eine positive Einstellung zu Integration sowie die Kommunikationsbereitschaft und -fähigkeit beider Seiten. Somit kommt auch an dieser Stelle sprachlichen Fähigkeiten eine hohe Bedeutung zu (Strahle/Baur 2009: 143). 
Die Befragungen der Ansprechpartnerinnen und -partner sowie der Übungsleiterinnen und leiter wurden mit Hilfe einer quantitativen Befragung durchgeführt. Die Stichprobe umfasste dabei 336 Ansprechpersonen und 608 Übungsleiterinnen und -leiter, deren Angaben getrennt voneinander analysiert wurden, um die Gruppen miteinander vergleichen zu können (Baur/Mutz 2009: 160). Der Fragebogen enthielt insgesamt 10 Items zum allgemeinen Integrationsverständnis sowie drei Items, die sich auf Integration im Sport bezogen ${ }^{9}$. Diese sollten anhand einer vierstufigen Skala bewertet werden und wurden im Rahmen der Analyse in insgesamt drei Skalen, zu Orientierung am Aufnahmeland, Verleugnung der kulturellen Herkunft und Multikulturalismus, zusammengefasst. Die ersten beiden Skalen stehen dabei für assimilative Konzepte, während die Skala zu Multikulturalismus pluralistische Einstellungen misst (Baur/Mutz 2009: 155/156).

Im Rahmen der Analyse zeigten sich keine großen Unterschiede zwischen Ansprechpersonen und Übungsleiterinnen und -leitern. Wie in der vorangegangenen Befragung der Landeskoordinatorinnen und -koordinatoren, konnte auch innerhalb dieser Befragung keine Favorisierung eines bestimmten Integrationskonzepts herausgestellt werden. Vielmehr wurden ebenfalls assimilative als auch pluralistische Integrationsvorstellungen unterstützt (Baur/Mutz 2009: 161). So zeigte sich, dass beide Gruppen die Aussage, dass Zugewanderte die deutsche Sprache sprechen und ihr Verhalten der deutschen Kultur anpassen sollten, unterstützen. Gleichzeitig wurde jedoch auch die Tradierung der Herkunftskultur als wichtiges Element angesehen. Zum Multikulturalismus zeigten sich geteilte Meinungen, mit der Tendenz zur Ablehnung dieses Konzepts. Darüber hinaus zeigte sich auch, dass viele Befragte mit „unentschieden“ antworteten. Dies könnte darauf verweisen, dass sich ein großer Teil der interviewten Personen noch nicht mit dem Thema Integration auseinandergesetzt hat (Baur/Mutz 2009: 169).

Insgesamt zeigt die Befragung der Evaluation des Programms „Integration durch Sport“ ein sehr undifferenziertes Bild des subjektiven Verständnisses der befragten Akteurinnen und Akteure. So lassen sich im Rahmen der Interviews assimilative, pluralistische sowie Mischungen aus beiden Ansätzen in den Ergebnissen erkennen (Vgl. Strahle/Baur 2009; Baur/Mutz 2009). Wo genau die Überschneidungen in diesen Konzepten liegen, lässt sich jedoch nicht herausstellen. Konsens zeigte sich bei den Landeskoordinatorinnen und koordinatoren zumindest in Bezug auf die Notwendigkeit eines „doppelseitigen

\footnotetext{
${ }^{9} \mathrm{Zu}$ den Items: Siehe Anhang. Im Folgenden werden nur die Ergebnisse zum Allgemeinen Integrationskonzept dargestellt, da diese auch in dieser Arbeit von Bedeutung sind.
} 
Integrationsbegriffs“. So müssen zum einen Zugewanderte Integrationswillen besitzen, wie auch Einheimische auf Zugewanderte zugehen und Akzeptanz zeigen (Strahle/Baur 2009: 149$151)$.

Auch abseits der Sportvereinsforschung ist das subjektive Integrationsverständnis von Bedeutung. So beschäftigt sich Schramkowski $(2007,2009)$ im Rahmen von Interviews mit Expertinnen, Experten und jungen Erwachsenen mit Migrationshintergrund mit dem subjektiven Integrationsverständnis.

Im Rahmen der Expertinnen- und Experteninterviews wurden acht Personen interviewt, die selbst einen Migrationshintergrund besitzen und Migrationsprozesse professionell begleiten oder in einem Migrantenverein aktiv sind (Schramkowski 2007: 147). Dabei wurde neben der Ausgestaltung gelungener Integration (u. a. Hindernisse, Rahmenbedingungen und Anforderungen an die Mehrheitsgesellschaft) auch über das persönliche Verständnis von Integration gesprochen (Schramkowski 2007: 135). Die Ergebnisse zeigten, dass die Expertinnen und Experten Integration als gleichberechtigtes und selbstverständliches Miteinander verstehen, das gemeinsam gestaltet wird. Daraus ergibt sich eine beidseitige Verantwortung von Personen mit und ohne Migrationshintergrund bei der Ausgestaltung von Integration (Schramkowski 2007: 154). Um diese beidseitige Verantwortung zu erreichen, müssen vor allem Mehrfachzugehörigkeiten anerkannt und ethnische Zugehörigkeiten ihre Bedeutung verlieren. Denn nach Meinung der interviewten Expertinnen und Experten wird der Begriff der Integration momentan eher dazu verwendet, ethnische Differenzierungen vorzunehmen und Zugehörigkeiten in Frage zu stellen. So wird der Begriff der Integration beispielsweise oft im Zusammenhang mit Problemen verwendet. Dies hemmt die gleichberechtigte Partizipation, auch weil Erfolge von Personen mit Migrationshintergrund wenig Aufmerksamkeit erhalten. Durch die negative Konnotation des Begriffs werden Personen mit Migrationshintergrund dann selbstverständlich „Integrationsprobleme“ zugeschrieben (Schramkowski 2007: 155).

Die Anerkennung von Mehrfachzugehörigkeiten wird wiederrum durch das Verständnis von Integration als einseitige Anpassungsleistung gehemmt. Darüber hinaus erläuterten die Expertinnen und Experten auch, dass in der Gesellschaft vorhandene Einstellungen Integrationsprozesse und auch den Erfolg von Integration beeinflussen (Schramkowski 2007: 150-152), was vor allem an dem folgenden Zitat aus einem der Interviews deutlich wird: „Da kann der Türke sich anstrengen, wie er will, wenn die Deutschen ihn nicht aufnehmen möchten, 
dann klappt das hier auch nicht, dann kann er sich hier nicht integrieren.“ (E3: 196ff.) (Schramkowski 2007: 152).

Kritisch äußerten sich die Expertinnen und Experten darüber hinaus bezüglich der Bewertung von Integration anhand von äußeren Maßstäben (Schramkowski 2007: 155). Beispielsweise wird im öffentlichen Diskurs und auch in der Literatur die Teilnahme an vereinsorganisierten Freizeitangeboten als Maßstab für das „Integriert sein“ herangezogen. Jedoch kann die fehlende Teilnahme an diesen Angeboten auch darauf zurückzuführen sein, dass Personen einfach nicht an diesen Angeboten teilnehmen möchten. Andere Bewertungskriterien führen beispielsweise dazu, dass Integration eine gewisse Relativität aufweist. So stellt das Bewertungskriterium „Teilhabe am Arbeitsmarkt“ Integration als einen nicht konstanten Zustand dar, weil im Falle der plötzlichen Arbeitslosigkeit dieses Kriterium nicht mehr erfüllt wäre (Schramkowski 2007: 148-149). Laut den Expertinnen und Experten sollte es bei Integration, statt um allgemeine Bewertungskriterien, vielmehr um das „Sich integriert fühlen“ gehen, was die Subjektivität von Integration betont (Schramkowski 2007: 149).

Neben den Expertinnen- und Experteninterviews führte Schramkowski (2009) auch 16 Interviews mit jungen Erwachsenen zwischen 19 und 26 Jahren zu den Themen Erfahrungen mit dem Integrationsprozess, Assoziationen mit dem Begriff Integration und individuelles Integrationsempfinden. Diese jungen Erwachsenen wurden durch die zuvor interviewten Expertinnen und Experten ausgewählt, in dem nach Personen gefragt wurde, deren Integration die Experten als positiv beurteilten (Schramkowski 2009: 150/151). Dabei wurde darauf geachtet, dass das Geschlechterverhältnis der befragten Personen ausgeglichen ist und die jungen Erwachsenen unterschiedliche familiäre Hintergründe und Bildungsabschlüsse aufweisen. Insgesamt besitzen acht der interviewten jungen Erwachsenen eine türkische Herkunft, sind aber in Deutschland geboren. Die anderen acht Personen gehören zur Gruppe der Spätaussiedler und kamen im Alter zwischen 9 und 18 Jahren nach Deutschland (Schramkowski 2009: 150).

Im Rahmen der Interviews zeigte sich, dass der Begriff der Integration überwiegend negativ konnotiert ist und im Verständnis der jungen Erwachsenen auch Unterschiede zu den in der Wissenschaft und Gesellschaft formulierten Kernelementen aufweist (Schramkowski 2009: 152). Die jungen Erwachsenen sehen Integration als eine einseitige Anpassungsleistung der Personen mit Migrationshintergrund an die deutsche Kultur. Weiter beschrieben die Interviewpartnerinnen und -partner, dass Integration von Personen ohne Migrationshintergrund oft alleinig mit dem Erwerb der deutschen Sprache gleichgesetzt wird. Somit stellt Integration 
Anforderungen an Personen mit Migrationshintergrund, während Personen ohne Migrationshintergrund kaum integrationsfördernde Rahmenbedingungen schaffen. Außerdem wird der Begriff der Integration im öffentlichen Diskurs, nach Meinung der jungen Erwachsenen, meist verwendet, wenn Defizite von Personen mit Migrationshintergrund diskutiert werden (Schramkowski 2009: 153/154).

Die jungen Erwachsenen verstehen Integration außerdem als eine Art „Trennungskonstrukt“, durch welches eine Differenzierung zwischen „Deutschen und Ausländern“ erst vorgenommen wird (Schramkowski 2009: 154). Darüber hinaus betonen die jungen Erwachsenen auch die Unbeständigkeit von Integration, da „Integriert sein“ vor dem Hintergrund gewisser öffentlicher Diskurse (bspw. Debatte um Islamismus) in Frage gestellt werden kann. Es ergibt sich somit keine selbstverständliche dauerhafte Zugehörigkeit (Schramkowski 2009: 155/156).

Insgesamt zeigt sich im Rahmen der Studie, dass die interviewten Expertinnen und Experten sowie die jungen Erwachsenen mit Migrationshintergrund einen kritischeren Blick auf den Begriff der Integration haben als die Befragten im Rahmen der Evaluation von Baur et al. (2009), in welcher ein vorhandener Migrationshintergrund der Befragten nicht ausgewiesen wurde. Dieser kritischere Blick ist möglicherweise vor allem vor dem Hintergrund persönlicher Erfahrungen entstanden. Neben der eigenen Sichtweise auf Integration beschreiben die Interviewpartnerinnen und -partner dabei auch immer wieder die momentane Ausgestaltung des Begriffs in der Gesellschaft und üben Kritik daran.

Lutter (2011) beschäftigt sich in seiner Veröffentlichung „Integration im Bürgerbewusstsein von SchülerInnen“" mit der Bedeutung von Integration aus der Perspektive von Schülerinnen und Schülern. Ziel der Untersuchung war dabei, durch die Berücksichtigung der Vorstellungen von Schülerinnen und Schülern empirisch basierte Lehr- und Lerndesigns zu entwickeln.

Die Analyse umfasste Interviews mit sechs Schülerinnen und Schülern zwischen 17 und 19 Jahren. Die Schülerinnen und Schüler besuchten eine Gesamtschule in Niedersachsen oder Nordrhein-Westfalen und wurden mit Hilfe einer Leistungsbewertung der Lehrkräfte ausgesucht, um eine Spannbreite zwischen leistungsstarken und nicht leistungsstarken Schülerinnen und Schülern abzubilden. Darüber hinaus wurde das politische Interesse für die Auswahl miteinbezogen und einige der Befragten besitzen einen Migrationshintergrund (zweite oder dritte Generation) (Lutter 2011: 96/97). Die Analyse der Interviews wurde mit Hilfe einer Modifikation der qualitativen Inhaltsanalyse im Kontext fachdidaktischer Lehr- und Lernforschung vorgenommen (Lutter 2011: 18). 
Im Rahmen der Untersuchung wurde vor allem die Frage, wie sich Schülerinnen und Schüler eine ,gelungene Integration“, vorstellen fokussiert (Lutter 2011: 87). Es zeigte sich, dass das Konzept der Integration von allen Interviewten als Anpassung verstanden und dadurch negativ gesehen oder abgelehnt wird. Dabei wurde durchgängig eine sozial-kulturelle Perspektive von Integration deutlich, die auf Aspekte wie den Erwerb von Sprachkenntnissen und notwendigem Basiswissen ausgerichtet ist (Lutter 2011: 190). Aspekte, die die strukturelle Integration betreffen, wurden von den Schülerinnen du Schülern hingegen nicht thematisiert (Lutter 2011: 196). Innerhalb der individuellen Konzepte konnten funktional-intentionale und multikulturelle Orientierungen unterschieden werden, innerhalb derer unterschiedliche Positionierungen zur Anpassung. Im Rahmen der funktional-intentionalen Orientierungen variieren die Aussagen zwischen Legitimierung und Ablehnung der Anpassung an sozial-kulturelle Alltagsstandards. So erläutern manche Befragte, dass im Rahmen der sozial-kulturellen Ebene eine Anpassung aufgrund der Funktion für die Bewältigung des Alltags befürwortet werden kann. Bei Befragten mit multikulturellen Orientierungen, bei denen eine mehr normative Ausrichtung deutlich wurde, indem diese beispielsweise den Wert kultureller Pluralität betonten, zeigte sich hingegen eine durchgängige Ablehnung von Anpassungsvorstellungen (Lutter 2011: 190). Darüber hinaus führt Lutter aus, dass Ein- und Ausgrenzungsmechanismen nur partiell hinterfragt wurden und die Vorstellungen der Schülerinnen und Schüler sich stark an Konzepten des Deutschseins und Nicht-Deutschseins sowie des Integriertseins und Nichtintegriertseins orientierten (Lutter 2011: 191).

Ebenfalls in der Schulforschung beschäftigt sich Hanhart (2020) mit dem Thema Migration in den Vorstellungen von Grundschulkindern. Ziel der Arbeit war die Erarbeitung von Leitlinien für den Unterricht zum Thema Migration. Im Rahmen dieser Arbeit ergeben sich jedoch ebenfalls Bezüge zu den Vorstellungen von Integration.

Die Analyse umfasste Interviews mit 17 Schülerinnen und Schülern der Klassenstufen drei und vier in Nordrhein-Westfalen, in deren Klassen Migration als Lerngegenstand noch nicht behandelt wurde. Insgesamt 12 der interviewten Schülerinnen und Schüler besitzen einen Migrationshintergrund und zwei davon eine eigene Migrationserfahrung (Hahnhart 2020: 155157). Es zeigte sich, dass der Begriff der Integration für einige Kinder Teil des Wortschatzes ist und sie eigene Vorstellungen zu dessen Bedeutung besitzen (Hahnhart 2020: 407). Eine Person beschrieb Integration dabei als das Gewöhnen an andere Bedingungen (Hanhart 2020: 225). Darüber hinaus wurde ein prozesshafter Charakter von Integration deutlich, der mit einer Veränderung der Lebensgewohnheiten einhergeht (Hanhart 2020: 228). 
Neben diesen expliziten Ausführungen zum Begriff der Integration wurden jedoch auch in den weiteren Ergebnissen Aspekte deutlich, die in Bezug zu einem Verständnis von Integration stehen. So stellten alle Kinder zunächst dar, dass es nach der Einwanderung in ein anderes Land zu Veränderungen kommt und die Personen nicht mehr so leben wie in ihrem Herkunftsland. Dabei wird erneut eine gewisse Prozesshaftigkeit deutlich (Hanhart 2020: 367/368). Darüber hinaus zeigte sich bei der Behandlung der Frage, welche Bedingungen und Verhaltensweisen die Schülerinnen und Schüler als sinnvoll ansehen, damit sich Zugewanderte im Land wohlfühlen können und zurechtkommen, Aspekte genannt werden, die auch im Integrationsprozess von Bedeutung sind. So nennen die Schülerinnen und Schüler die Notwendigkeit der Abwesenheit von Ausländerfeindlichkeit und Diskriminierung (Hanhart 2020: 369) als auch die Unterstützung und konkrete Hilfen für Zugewanderte. Diese umfassen in der Vorstellung der Schülerinnen und Schüler Hilfen, wenn etwas nicht verstanden wird, sowie Hilfen beim Erlernen der neuen Sprache und der Orientierung in der neuen Umgebung (Hanhart 2020: 370). Das Erlernen der Sprache spielt für die Kinder auch bei der Frage, was zugewanderte Kinder konkret tun können, um im Land zurechtzukommen und sich wohlzufühlen, eine Rolle. Darüber hinaus werden hier auch soziale Bindungen und Freundschaften als wichtige Aspekte genannt (Hanhart 2020: 370).

\subsubsection{Der Zusammenhang von subjektiven Sichtweisen auf Integration und sozialstrukturellen Merkmalen}

Neben dem subjektiven Verständnis von Integration soll in dieser Arbeit außerdem der Frage nachgegangen werden, welche Zusammenhänge zwischen sozialstrukturellen Merkmalen und bestimmten Ansichten von Integration bestehen. Dazu wird im Folgenden erneut auf die Evaluation des Programms „Integration durch Sport“ eingegangen, im Rahmen dessen Baur und Mutz (2009) auch Zusammenhänge zwischen sozialstrukturellen Merkmalen der Befragten sowie strukturellen Merkmalen der Vereine und der subjektiven Sichtweise auf Integration untersucht haben. Im Anschluss daran wird ein Überblicksartikel von Ceobanu und Escandell (2010) vorgestellt, in dem unter anderem Indikatoren, die im Zusammenhang mit der Einstellung gegenüber Zugewanderten und Migration stehen ${ }^{10}$, beschrieben werden. Aufgrund der Nähe der Themen Integration und Migration wird vermutet, dass diese Indikatoren auch einen Zusammenhang mit der Einstellung zu Integration aufweisen. Die Indikatoren aus der

\footnotetext{
${ }^{10} \mathrm{Im}$ Folgenden wird nur auf individuelle Faktoren eingegangen. Die in der Arbeit betrachteten kontextuellen Faktoren haben für die vorliegende Arbeit keine Bedeutung.
} 
Einstellungsforschung sollen somit das Set möglicher Einflussfaktoren auf subjektive Sichtweisen auf Integration erweitern.

In Bezug auf subjektive Sichtweisen auf Integration gibt es, wie zuvor dargestellt, wenig Literatur. Auch Zusammenhänge zwischen sozialstrukturellen Merkmalen und bestimmten Ansichten von Integration wurden bisher in der Literatur vernachlässigt. Baur und Mutz (2009) ziehen in ihrer bereits vorgestellten Arbeit zur Evaluation des Programms „Integration durch Sport" unterschiedliche mögliche Einflussfaktoren heran, die die subjektiven Integrationskonzepte von Ansprechpartnerinnen und -partnern sowie von Übungsleiterinnen und -leitern beeinflussen könnten. Zur Analyse wurden dabei ebenfalls die bereits vorgestellten Items zum Integrationskonzept verwendet ${ }^{11}$.

Die möglichen Faktoren, die einen Zusammenhang mit der Unterstützung von Integrationskonzepten aufweisen könnten, umfassen bei Baur und Mutz sozialstrukturelle Merkmale der Befragten, Tätigkeitsmerkmale im Verein und Merkmale der Sportgruppen (Baur/Mutz 2009: 159). Sozialstrukturelle Merkmale sind dabei Alter, Geschlecht und Migrationshintergrund. In Bezug auf das Alter wird angenommen, dass vor allem jüngere Befragte eine eher pluralistische Vorstellung besitzen, während ältere Befragte assimilative Konzepte favorisieren. Das Geschlecht soll insofern einen Einfluss besitzen, dass Frauen ein pluralistisches Konzept eher unterstützen. Darüber hinaus soll eine vorhandene Migrationserfahrung die Einstellung hin zur Unterstützung pluralistischer Konzepte färben (Baur/Mutz 2009: 159).

In Bezug auf Tätigkeitsmerkmale werden die Indikatoren Dauer der Tätigkeit der Übungsleiterinnen und -leiter, Qualifikationen und Teilnahme an der Weiterbildung „Sport Interkulturell“ herangezogen. Eine längere Tätigkeitsdauer, Qualifikationen und die Teilnahme an der Weiterbildung „Sport Interkulturell“ sollen eine eher pluralistische Einstellung fördern (Baur/Mutz 2009: 159).

Als Merkmal der Sportgruppe wird außerdem die Konstellation der Teilnehmenden, in Form des Anteils von Zugewanderten, herangezogen. Hier soll ein höherer Anteil von Zugewanderten zu eher pluralistischen Einstellungen führen, da durch die vermehrten Kontakte bei einem höheren Anteil von Zugewanderten in der Sportgruppe mit einer „Liberalisierung“ von Integrationsvorstellungen zu rechnen ist (Baur/Mutz 2009: 167).

\footnotetext{
${ }^{11}$ Siehe Unterkapitel 3.2.1 „Forschungsarbeiten zu subjektiven Sichtweisen auf Integration“ und zu den Items und Skalen Anhang S. 208/209.
} 
Es zeigt sich, dass lediglich das Alter, eine vorhandene Migrationserfahrung und die Konstellation der Teilnehmenden der Trainingsgruppe signifikante Zusammenhänge mit der Einstellung zu Integrationskonzepten aufweisen (Baur/Mutz 2009: 163-168).

So stimmen ältere Ansprechpartnerinnen und -partner sowie Übungsleiterinnen und -leiter signifikant häufiger der Skala „Orientierung am Aufnahmeland“ zu als jüngere Befragte der beiden Gruppen (Baur/Mutz 2009: 163). In Bezug auf den Indikator Migrationserfahrung zeigt sich, dass die Ansprechpartnerinnen und -partner mit eigener Migrationserfahrung eine höhere Zustimmung bei der Skala „Verleugnung der kulturellen Herkunft“ aufweisen, was auf einen erlebten Integrationsdruck zurückzuführen sein könnte. Übungsleiterinnen und -leiter mit Migrationshintergrund, die somit auch Personen ohne eigene Migrationserfahrung umfassen, stimmen dem Konzept des Multikulturalismus signifikant häufiger zu als Übungsleiterinnen und -leiter ohne Migrationshintergrund (Baur/Mutz 2009: 164). Der letzte Faktor, der im Rahmen der Analyse einen signifikanten Zusammenhang aufweist, ist die Konstellation der Teilnehmenden. Hier zeigt sich, dass hohe Anteile an Zugewanderten in der Sportgruppe dazu führen, dass eine höhere Zustimmung für die Multikulturalismus-Skala vorliegt (Baur/Mutz 2009: 168). Dabei ist jedoch zu beachten, dass es sich bei den zugrunde gelegten Anteilen an Zugewanderten in den Sportgruppen um eine Einschätzung durch die Befragten handelt, welche somit lediglich dem wahrgenommenen und nicht dem tatsächlichen Anteil entspricht.

Da in Bezug auf Zusammenhänge zwischen sozialstrukturellen Merkmalen und subjektiven Integrationsvorstellungen, wie vorgestellt, nur wenig Literatur vorliegt, soll im Rahmen dieser Arbeit auch auf Indikatoren zurückgegriffen werden, die in der Einstellungsforschung im Zusammenhang mit der Einstellung gegenüber Zugewanderten und Migration stehen.

In einem Überblicksartikel haben Ceobanu und Escandell (2010) unterschiedliche Arbeiten gegenübergestellt und dabei auch die sozialstrukturellen Einflussfaktoren betrachtet. Die Datensätze der dargestellten Studien sind der Eurobarometer, das International Social Survey Program und der European Social Survey. Daher beziehen sich die Arbeiten auch auf europäische Staaten und dabei überwiegend auf Westeuropa (Ceobanu/Escandell 2010: 312). Im Rahmen der Gegenüberstellung gehen Ceobanu und Escandell auf die Faktoren individuelle Bildung, sozialer Status, Alter, Geschlecht und Wohnort ein.

Die individuelle Bildung weist in vielen Arbeiten einen stabilen Zusammenhang mit der Einstellung gegenüber Zugewanderten und Migration auf. Dabei weisen Personen, mit höherer Bildung eine positivere Einstellung gegenüber Zugewanderten und Migration auf als Personen mit niedrigerer Bildung. Mögliche Erklärungen für diesen Zusammenhang ist zum einen ein liberalisierender Effekt von Bildung, durch beispielsweise ein höheres Wissen. Zum anderen 
werden beispielsweise eine kritischere Haltung und eine größere Akzeptanz von Diversität zur Begründung herangezogen. Darüber hinaus wird in der Arbeit darauf hingewiesen, dass der Effekt der Bildung deutlicher wird, wenn die Bildung statt in Schuljahren in Kategorien abgefragt wird und dass sich Unterschiede zwischen osteuropäischen und westeuropäischen Staaten zeigen, welche auf Unterschiede im nationalen Bildungssystem und auf unterschiedliche Sozialisation zurückzuführen sind (Ceobanu/Escandell 2010: 319).

Der soziale Status hat vor allem in Form der Stellung im Erwerbsleben und des beruflichen Status einen Einfluss auf die Einstellung gegenüber Zugewanderten und Migration. Personen mit einem niedrigeren sozialen Status weisen dabei eine negativere Einstellung gegenüber Zugewanderten und Migration auf als Personen, denen anhand dieser beiden Indikatoren ein höherer sozialer Status zugeschrieben wird. Dies wird vor allem damit begründet, dass Personen in einer niedrigeren sozialen Stellung befürchten, im Wettbewerb mit Zugewanderten mit einem ähnlichen sozialen Status zu stehen. Auch in Bezug auf diese Indikatoren zeigt sich in westeuropäischen Staaten eine höhere Voraussagekraft als in osteuropäischen Staaten. Darüber hinaus wird angeführt, dass Kontextfaktoren, wie eine generell erhöhte Arbeitslosigkeit, diesen Zusammenhang beeinflussen könnten. Im Gegensatz zur Stellung im Erwerbsleben und dem beruflichen Status weist das individuelle Einkommen nicht so häufig einen Zusammenhang mit der Einstellung gegenüber Zugewanderten und Migration auf (Ceobanu/ Escandell 2010: 319/320).

Weitere mögliche Einflussfaktoren, die in Studien herangezogen werden, sind Alter, Geschlecht und Wohnort (städtisch oder ländlich). Diese demografischen Indikatoren weisen jedoch einen weniger stabilen Zusammenhang mit der Einstellung gegenüber Zugewanderten und Migration auf. Dennoch kommen einige Studien zu dem Ergebnis, dass ältere männliche Befragte aus ländlichen Regionen eine negativere Einstellung gegenüber Zugewanderten und Migration besitzen (Ceobanu/Escandell 2010: 320).

\subsubsection{Einordnung des Forschungsstandes zu subjektiven Sichtweisen auf Integration} In Bezug auf den Forschungsstand zu subjektiven Sichtweisen auf Integration kann zunächst festgehalten werden, dass Arbeiten aus unterschiedlichen Themenbereichen eine Rolle spielen können, welche nicht immer direkt subjektive Sichtweisen auf Integration betrachten. Diese umfassen Arbeiten zur Einstellung zu Ausländerinnen und Ausländern, Integration und Akkulturation (Van Dick et al. 1997; Zick/Preuß 2016; Blohm/Wasmer 2018) sowie Arbeiten, die die Unterstützung von Integrationskonzepten zum Thema haben (Strahle/Baur 2009, Baur/Mutz 2009; Hachfeld et al. 2011, 2012, 2015; Civitillo et al. 2017, 2019). Darüber hinaus 
existieren Arbeiten, die sich mit dem Integrationsverständnis beschäftigen (Lutter 2011; Hanhart 2020; Schramkowski 2007, 2010).

Die Arbeit von Blohm und Wasmer (2018) zeigte, dass das Konzept der Assimilation in der Bevölkerung großen Zuspruch findet und sich die Bewertung des Konzepts zwischen den Bildungsgruppen, auf deskriptiver Ebene, unterscheidet (vgl. Blohm/Wasmer 2018). In der Arbeit von Zick und Preuß zeigte sich hingegen, dass das Konzept der Integration, im Sinne der Mehrfachintegration, die höchste Zustimmung aufwies. Dabei zeigten sich auch signifikante Unterschiede zwischen Personen mit und ohne Migrationshintergrund (Zick/Preuß 2016: 16). Die Arbeiten kommen somit, obwohl ähnliche Integrationskonzepte abgefragt werden zu unterschiedlichen Ergebnissen. Während Blohm und Wasmer jedoch die Einstellung $\mathrm{zu}$ Ausländerinnen und Ausländern anhand von Fragestellungen aus dem ALLBUS untersuchten, beschäftigten sich Zick und Preuß mit Einstellungen der Bevölkerung zu Integration allgemein. Dies zeigt, dass Integrationskonzepte je nach Fragestellung und Forschungskontext unterschiedlich interpretiert werden können.

Die Arbeit von Hachfeld et al. (2015) zeigte darüber hinaus, dass Zusammenhänge zwischen Überzeugungen und beruflichen Kompetenzen von angehenden Lehrenden für das Unterrichten von Schülerinnen und Schülern mit Migrationshintergrund existieren (vgl. Hachfeld et al. 2015). Außerdem ist im schulischen Kontext ein vorhandener Migrationshintergrund von Lehrenden vielleicht weniger entscheidend für professionelle Kompetenzen im Kontext kultureller Heterogenität als multikulturelle Überzeugungen an sich (Hachfeld et al. 2012: 114/115). Bei Civitillo et al. (2019) zeigte sich eine hohe Kongruenz zwischen kultursensiblem Unterricht und Überzeugungen zu kultureller Vielfalt. Diese Ergebnisse zeigen, dass die Einstellung der Akteurinnen und Akteure eine bedeutende Rolle im Miteinander spielt, womit im Rahmen der Integration deren Verständnis sowie die Einstellung zu Integration relevant werden.

Im Rahmen der Evaluation von Baur et al. (2009) zeigte sich ein sehr undifferenziertes Bild des subjektiven Integrationsverständnisses der befragten Akteurinnen und Akteure. Darüber hinaus zeigte sich auch, dass sich Vorstellungen zu Integration im Allgemeinen und in Bezug auf den Sport nicht immer trennscharf unterscheiden lassen. Das undifferenzierte Bild des subjektiven Verständnisses der Landeskoordinatorinnen und -koordinatoren in der Studie von Baur et al. könnte auf die offene Fragegestaltung in den Interviews zurückzuführen sein. Die direkte Frage danach, was Integration für die einzelne Person bedeutet, scheint zu komplex zu sein. Aus diesem Grund ist bei manchen Befragten auch die Einführung von Statements zur Anregung 
weiterer Überlegungen nötig. Außerdem zeigt sich, dass sich die Antworten der Landeskoordinatorinnen und -koordinatoren stark auf Ergebnisse von Integration, in Form von assimilativen oder pluralistischen Tendenzen, beziehen und die Elemente von Integration nur wenig aufgezeigt werden.

Bei der quantitativen Befragung der Ansprechpartnerinnen und -partner sowie der und Übungsleiterinnen und -leiter zeigt sich, dass viele Befragte mit „unentschieden“ antworten. Dies kann darauf verweisen, dass sich ein großer Teil der Interviewten noch nicht mit dem Thema Integration auseinandergesetzt hat (Baur/Mutz 2009: 169). Andererseits kann auch dadurch wieder die Komplexität der Frage deutlich werden.

Weiter ist anzumerken, dass die vorgestellte Evaluation nur auf die Funktionärsebene der Sportorganisationen beschränkt ist, obwohl zugleich dargestellt wird, dass auch die Erfahrungen in Sportgruppen die Ansichten der Übungsleiterinnen und -leiter färben können (Baur/Mutz 2009: 158). Aus diesem Grund sind auch die Mitglieder von Vereinen als relevante Akteurinnen und Akteure anzusehen. Zwar ist das subjektive Verständnis der Funktionärsebene auf einer politischen Ebene von Bedeutung, die tatsächliche Interaktion findet aber zwischen den Trainingsleitungen und Mitgliedern statt.

In den Arbeiten von Schramkowski (2007, 2009) fällt insgesamt der starke Fokus auf das Verständnis von Integration vor dem Hintergrund persönlicher Erfahrungen auf. Dies könnte auf die Verknüpfung der Forschungsfragen zu Verständnis von Integration und Erlebnis der eigenen Integration zurückzuführen sein. Aus diesem Grund ist das Verständnis von Integration stark vor dem Hintergrund der persönlichen Erfahrung mit Integration verbunden und die Befragten wechseln des Öfteren zwischen einer Beschreibung dessen, was sie persönlich unter Integration verstehen und der Beschreibung dessen, wie Integration in der Gesellschaft ausgestaltet wird. So verstehen die Expertinnen und Experten unter Integration ein gleichberechtigtes und selbstverständliches Miteinander, das gemeinsam gestaltet wird (Schramkowski 2009: 154). Kritisch sehen die Expertinnen und Experten jedoch die Verwendung des Begriffs in der Gesellschaft, da dieser dort beispielsweise zu ethnischen Differenzierungen führt und überwiegend in Zusammenhang mit Problemen thematisiert wird (Vgl. Schramkowski 2007). Die Trennung zwischen diesen beiden Perspektiven wird bei Schramkowksi nicht deutlich und führt dazu, dass ein negatives Verständnis des Integrationsbegriffs angenommen wird.

Die befragten jungen Erwachsenen verstehen Integration als einseitige Anpassungsleistung (Schramkowski 2009: 153). Auch innerhalb der Perspektiven der Jugendlichen wird nicht 
eindeutig zwischen ihrem tatsächlichen Verständnis von Integration und der Kritik an der Ausgestaltung in der Gesellschaft (bspw. Integration als Trennungskonstrukt und Unbeständigkeit der Integration) unterschieden.

Ähnlich wie in den Arbeiten von Schramkowski, zeigt sich auch bei Lutter (2011) eine negative Konnotation des Konzepts der Integration, das stark mit einer Anpassungsvorstellung verbunden ist. Auch hier liegt der Fokus der Studie eher auf Ausgängen von Integration im Sinne einer „gelungenen Integration“ und Aspekte von Integration treten in den Hintergrund. Im Gegensatz zur Arbeit von Schramkowski beschränkt sich die Arbeit von Lutter nicht nur auf Personen mit Migrationshintergrund, sondern beinhaltet sowohl Interviews mit Schülerinnen und Schülern mit Migrationshintergrund als auch ohne. Dennoch findet kein systematischer Vergleich der eventuell unterschiedlichen Perspektiven von Personen mit und ohne Migrationshintergrund statt.

Die Ergebnisse der Arbeit von Hanhart (2020) zeigen zudem, dass schon früh Vorstellungen über Integration vorhanden sind, die sich über die Zeit weiterentwickeln und verfestigen können. Hanhart führt dazu aus, dass es dabei in der Schule relevant werden kann, das Verständnis von Integration $\mathrm{zu}$ erweitern. Dies ist möglich, indem neben dem Alltagsverständnis, im Rahmen dessen Integration oft als Anpassung verstanden wird, auch auf Vorstellungen des theoretischen Diskurses verwiesen wird, die Integration als gemeinsame Aufgabe beschreiben (Hanhart 2020: 407).

Für die vorliegende Arbeit können abschließend drei Punkte aus der Betrachtung des Forschungsstandes zu subjektiven Sichtweisen auf Integration herausgearbeitet werden.

Die Darstellung der bisherigen Arbeiten bezüglich der subjektiven Sichtweisen auf Integration zeigt zunächst, dass ein starker Fokus auf Ergebnissen von Integration bzw. der Untersuchung von favorisierten Integrationskonzepten besteht, während Vorstellungen über die konkrete Ausgestaltung eher in den Hintergrund treten. So werden beispielsweise konkrete Kriterien der Zugehörigkeit sowie die Vorstellungen zum Zusammenleben nur bei Zick und Preuß (2016) behandelt. Hier zeigte sich in beiden Befragungen, dass vor allem die Achtung politischer Institutionen und Gesetze, die deutsche Sprache, das Gefühl sich in Deutschland Zuhause zu fühlen und die Erwerbstätigkeit hohe Relevanz besitzen (Zick/Preuß 2016: 11-13).

Einzelne aus Sicht der Befragten relevante Aspekte, wie das Erlernen der Sprache, werden in weiteren Arbeiten zwar immer wieder herausgestellt, ein Vergleich mit der Bedeutung anderer Aspekte aus dem theoretischen Diskurs oder wie diese miteinander in Beziehung stehen, fehlt 
jedoch. Dadurch zeigt sich, dass Ergebnisse und Dimensionen/Aspekte von Integration getrennt $\mathrm{zu}$ sehen sind und gerade Ergebnisse von Integration oft eine normative Prägung aufweisen, weil dort ein Bezug zu ,gelungener Integration“ hergestellt wird. Es geht bei Ergebnissen von Integration somit um die Ausgänge, die als erstrebenswert erscheinen, während Dimensionen/Aspekte von Integration auch als analytisches Konstrukt für die Messung des „Integriertseins“ gesehen werden können. Lutter (2011) führt hierzu aus: „Wird der vermeintlichen Normativität des Integrationsbegriffs ein programmatisch-fachliches Profil im Sinne einer analytischen Differenzierung verliehen, so entwickelt er durchaus Erklärungskraft und trägt einer differenzierten Annäherung an die Voraussetzungen, Bedingungen und Herausforderungen des Zusammenlebens zwischen zugewanderten, ethnisch-kulturellen Minderheiten und der Mehrheitsgesellschaft Rechnung“ (Lutter 2011: 50). In der vorliegenden Arbeit sollen beide Seiten und somit die Ergebnisse und die Dimensionen/Aspekte von Integration beleuchtet werden, um so den Begriff der Integration im Sinne der erstrebenswerten Ergebnisse als auch seiner Ausgestaltung zu beleuchten.

Ein weiterer Punkt betrifft die für die Untersuchung bedeutenden Akteurinnen und Akteure: Zunächst zeigt sich, dass nicht nur Funktionäre sowie Trainerinnen und Trainer eine Rolle für die subjektiven Sichtweisen auf Integration in den Vereinen spielen, sondern auch die Sichtweisen der Mitglieder Bedeutung haben könnten. Denn auch die Erfahrungen in den Sportgruppen können die Ansichten der Übungsleiterinnen und -leiter färben (Baur/Mutz 2015: 158). Neben der Differenzlinie der unterschiedlichen Akteurinnen und Akteure in Sportorganisationen ist eine weitere interessante Differenzlinie im Verständnis von Integration diejenige, zwischen Personen mit und ohne Migrationshintergrund. So haben die Arbeiten von Schramkowski $(2007,2009)$ gezeigt, dass das Verständnis von Integration durch die eigene Integrationserfahrung geprägt werden kann, was bei den interviewten jungen Erwachsenen zu einer negativen Besetzung des Integrationsbegriffs geführt hat. Somit soll ein Fokus dieser Arbeit, neben den unterschiedlichen Akteursebenen, auf der Gegenüberstellung der Perspektiven von Personen mit und ohne Migrationshintergrund liegen. Diese Gegenüberstellung stellt in der bisherigen Forschung eine Lücke dar, die mit Hilfe dieser Arbeit gefüllt werden soll. Denn gerade die Perspektive von Personen mit Migrationshintergrund ist von großer Bedeutung, um den Diskurs um Integration nicht zu einem Gegenstand zu machen, der, wie Schramkowksi (2009) formuliert, überwiegend aus der Perspektive der deutschen Mehrheitsgesellschaft dargestellt wird. Darüber hinaus wird „dabei vor allem über Personen mit Migrationshintergrund gesprochen, anstatt miteinander über Ziele der Integration zu diskutieren und Handlungsstrategien gemeinsam festzulegen“ (Schramkowksi 2009: 149). 
Der dritte Punkt ist die Komplexität der Befragung. So hat sich gezeigt, dass eine offene Fragestellung, in der nach der persönlichen Bedeutung von Integration gefragt wird, zum Teil von den Befragten nicht beantwortet werden konnte. Selbst bei den Landeskoordinatorinnen und -koordinatoren des Programms „Integration durch Sport“ entstanden bei dieser Fragestellung Probleme, obwohl bei diesen Personen eine gewisse Sensibilität für die Thematik $\mathrm{zu}$ erwarten ist. Der Fokus der Arbeiten lag darüber hinaus eher auf der Einstellung zu bestimmten Ergebnissen von Integration. Zwar wurden bei Schramkowksi (2007, 2009) einzelne Elemente von Integration angesprochen, diese stellten teilweise aber eher eine Kritik an der Ausgestaltung von Integration in der Gesellschaft (bspw. starker Fokus auf Spracherwerb) dar, als Elemente, die die Befragten als wichtige Elemente von Integration empfinden. Was fehlt ist somit eine Untersuchung des tatsächlichen Verständnisses von Integration und damit einhergehend, welche Aspekte und Dimensionen von Integration Bedeutung haben. Diese Untersuchung sollte losgelöst von Ergebnissen von Integration sowie der Unterstützung bestimmter Integrationskonzepte erfolgen. Aus diesem Grund sollen in dieser Arbeit die Aspekte von Integration (bspw. Erlernen der Sprache) und die Ausgänge von Integration (Favorisierung assimilativer oder pluralistischer Einstellungen) getrennt voneinander behandelt und erfragt werden. Zu diesem Zweck soll eine Fragebatterie entwickelt werden, mit Hilfe derer unterschiedliche Aspekte von Integration in ihrer Bedeutung bewertet werden können. Diese Fragebatterie wird aus dem wissenschaftlichen Diskurs zum Integrationsbegriff abgeleitet. Eine weitere Fragebatterie greift dann Aussagen in Bezug auf Ausgänge von Integration auf. Darüber hinaus liegt dieser Arbeit ein Mixed-Methods-Ansatz zugrunde, im Rahmen dessen zunächst eine quantitative Befragung durchgeführt wird, deren Ergebnisse anschließend mit qualitativen Interviews vertieft werden sollen ${ }^{12}$.

In Bezug auf die Zusammenhänge von Sichtweisen auf Integration und anderen Merkmalen hat die Arbeit von Baur und Mutz (2009) gezeigt, dass vor allem sozialstrukturelle Merkmale einen Einfluss auf die Einstellung gegenüber Integrationskonzepten, in Bezug auf Ausgänge von Integration, haben. Von den drei untersuchten sozialstrukturellen Merkmalen weisen das Alter und der Migrationshintergrund einen Zusammenhang mit subjektiven Sichtweisen auf Integration auf. Interessant ist hierbei, dass, wie bereits in den vorgestellten Studien zu subjektiven Sichtweisen auf Integration, erneut der Migrationshintergrund ein wichtiger Faktor zu sein scheint. Im Gegensatz dazu zeigt sich, dass Tätigkeitsmerkmale in der Sportorganisation keine Rolle spielen. Lediglich die Konstellation der Teilnehmenden der Sportgruppe weist

\footnotetext{
${ }^{12}$ Siehe hierzu Kapitel 5 ,Daten und Methoden“.
} 
ebenfalls einen Zusammenhang auf. Wie in der Forschung zu Einstellungen gegenüber Zugewanderten und Migration, weisen also auch hier sozialstrukturelle Merkmale einen Effekt auf.

In der vorgestellten Arbeit zu Einflussfaktoren auf die Einstellung gegenüber Zugewanderten und Migration sind vor allem die Merkmale individuelle Bildung, Stellung im Erwerbsleben, beruflicher Status und Alter von Bedeutung. Der Indikator individuelle Bildung soll auch in der vorliegenden Arbeit herangezogen und um die von Baur und Mutz (2009) identifizierten Faktoren Alter, Migrationshintergrund und die Konstellation der Teilnehmenden der Sportgruppe ergänzt werden. Die Stellung im Erwerbsleben und der berufliche Status werden aufgrund von eher jungen Befragten innerhalb der Stichprobe nicht herangezogen.

Auch wenn der Wohnort (städtisch oder ländlich), das Geschlecht und das Einkommen in manchen Arbeiten der Einstellungsforschung Zusammenhänge mit der Einstellung zu Zugewanderten und Migration aufweisen, werden diese Indikatoren in der vorliegenden Arbeit nicht herangezogen. Eine Unterscheidung nach städtischem oder ländlichem Wohnort ist im Rahmen der Erhebung nicht möglich, da diese sich nur auf Frankfurt und damit einen städtischen Bereich bezieht. In Bezug auf das Geschlecht ergeben sich in der Hinsicht Probleme, dass gerade Frauen und Mädchen mit Migrationshintergrund in Sportorganisationen deutlich unterrepräsentiert sind. Da der Faktor Geschlecht in der bisherigen Forschung darüber hinaus keinen konstanten Zusammenhang mit der Einstellung gegenüber Zugewanderten und Migration aufweist, wird dieser Faktor für diese Arbeit nicht herangezogen. Das Einkommen weist nach bisheriger Forschung ebenfalls keinen konstanten Zusammenhang mit der Einstellung gegenüber Zugewanderten und Migration auf. Darüber hinaus ist die Abfrage des individuellen oder Haushaltseinkommen immer mit vielen fehlenden Werten verbunden, da viele Befragte dieses nicht angeben wollen. Aus diesen Gründen soll auch das Einkommen nicht als Einflussfaktor in die späteren Analysen eingehen.

\section{Theoretische Konzepte von Integration}

Wie im Rahmen des Forschungsstandes herausgearbeitet, wird in der vorliegenden Arbeit der Begriff der Integration auf zwei unterschiedlichen Ebenen, die voneinander abgegrenzt werden sollen, betrachtet. Diese Perspektiven sind zum einen Ergebnisse von Integration und zum anderen Dimensionen und Aspekte von Integration. Neben der Untersuchung wünschenswerter Ausgänge von Integration, die in der bisherigen Forschung fokussiert werden, sollen so auch die Vorstellungen über die Ausgestaltung von Integration, in Form von Dimensionen und wichtigen Aspekten, herausgearbeitet werden. 
Zu Beginn des Kapitels werden Konzepte zu Ergebnissen von Integration dargestellt. Die in der Literatur am häufigsten diskutierten Konzepte sind dabei Assimilation und Pluralismus, deren Grundzüge in einem Unterkapitel dargestellt werden. Diese Konzepte bildeten auch schon im Forschungsstand den Schwerpunkt im Rahmen der Ausgänge von Integration. Darüber hinaus wird auch eine Arbeit von Esser (2001) vorgestellt, innerhalb derer vier verschiedene Ausgänge von Integration unterschieden werden. Diese Ausgänge sollen ergänzend zu den Konzepten Assimilation und Pluralismus hinzugezogen werden, um unterschiedliche Ausgänge zu operationalisieren bzw. um diese zu konkretisieren.

Im Anschluss daran werden in einem weiteren Unterkapitel unterschiedliche Dimensionen und Aspekte von Integration diskutiert, indem Arbeiten, die Dimensionen von Integration unterscheiden und diese mit einzelnen Aspekten der Integration versehen, vor- und im weiteren Verlauf gegenübergestellt werden. Im Allgemeinen ermöglichen solche Konzipierungen, tatsächliche Integrationsverläufe von bestimmten Gruppen zu betrachten. In der vorliegenden Arbeit soll mit Hilfe einer Gegenüberstellung unterschiedlicher Konzepte eine möglichst große Bandbreite an Aspekten für die Operationalisierung im Fragebogen herausgearbeitet werden. Auch wenn diese Konzepte teilweise auf mögliche oder wünschenswerte Ausgänge von Integration verweisen, soll in diesem Unterkapitel nur die Diskussion unterschiedlicher Dimensionen und Aspekte von Integration im Fokus stehen. 


\subsection{Ergebnisse von Integration}

In Bezug auf die Ergebnisse von Integration sind zunächst zwei Ebenen zu unterscheiden. Zum einen gibt es die empirische Ebene, die das Vorkommen unterschiedlicher Arten der Integration, im Rahmen tatsächlicher Integrationsverläufe, untersucht und auch beschreibt. Dabei kommt es nicht darauf an, ob ein bestimmtes Ergebnis von Integration favorisiert werden sollte und somit als wünschenswert gilt, sondern darauf, ob diese Ergebnisse von Integration empirisch nachgewiesen werden können (Hans 2016: 24). Zum anderen kann davon eine eher normative Ebene unterschieden werden, im Rahmen derer die Diskussion darüber stattfindet, welche Integrationsverläufe als wünschenswert angesehen werden (Nobis/Strahle 2009: 118).

Im Rahmen dieser Arbeit sollen in Bezug auf die Ergebnisse von Integration die Einstellungen der Befragten zu den Konzepten Assimilation, Pluralismus und den von Esser (2001) dargestellten Ausgängen abgefragt werden, indem die Zustimmung zu Aussagen, die auf die jeweiligen Ausgänge bezogen sind, gemessen wird. Die Messung der Zustimmung zu diesen Konzepten ist auf der normativen Ebene der Diskussion anzusiedeln, da es dabei darum geht, welches der Konzepte die Befragten als wünschenswert ansehen und nicht um die Abfrage tatsächlich vorliegender Integrationsverläufe. Aus diesem Grund soll innerhalb dieser Arbeit keine theoretische Auseinandersetzung damit stattfinden, welche Konzepte realistischer sind oder als wünschenswert angesehen werden sollten, da der Fokus dieser Arbeit darauf liegt, die subjektiven Sichtweisen der Personen in Sportvereinen zu untersuchen.

Für die Operationalisierung von Aussagen zu den Ergebnissen von Integration findet daher im anschließenden Unterkapitel eine Erläuterung der Grundzüge des Assimilations- und Pluralismus-Konzepts statt, sowie eine Darstellung der Entwicklung des theoretischen Diskurses von Assimilationsmodellen hin zu pluralistischen Ausgestaltungen.

Neben diesen beiden Ausgängen von Integration, sollen auch die vier möglichen Ausgänge nach Esser (2000) in einem weiteren Unterkapitel beschrieben werden. 


\subsubsection{Die Debatte um Assimilation und Pluralismus}

Die beiden am meisten diskutierten Konzepte, in Bezug auf Ergebnisse von Integration, bilden die Assimilation und der Pluralismus. Während Integration dabei als Oberbegriff zur Eingliederung in eine Gesellschaft verstanden werden kann, stellt sich im Rahmen dieser Debatte die Frage, ob diese Eingliederung durch einen Abbau von Unterschieden und Grenzziehungen (Assimilation) geschieht, oder, ob ethnische Subgruppen nebeneinander bestehen bleiben (Pluralismus).

Innerhalb der Integrationsforschung lag der Fokus lange Zeit auf der Assimilation. Assimilation enthielt dabei zum einen die Anforderung an Zugewanderte, sich an die Mehrheitsgesellschaft anzupassen. Zum anderen sollte diese Assimilation in einer gesellschaftlichen Homogenisierung enden (Strahle/Nobis 2009: 120). Dadurch kann Assimilation auf der einen Seite als Prozess der Anpassung und auf der anderen Seite als Ergebnis von Anpassung, die in einer Homogenisierung endet, verstanden werden. Darüber hinaus war bis in die 1950er Jahre auch die Einwanderungspolitik der USA, Kanada und Australien, die als traditionelle Einwanderungsländer gelten, durch die Vorstellung der Assimilation in Form von Anpassung geprägt (Han 2010: 294).

Eines der ersten Assimilationsmodelle, der „Race-relation-cycle“, entstand im Rahmen der Forschung der „Chicago School“ (Oswald 2007: 94). Park und Burgess beschreiben Assimilation dabei als ,a process of interpenetration and fusion in which persons and groups acquire the memories, sentiments, and attitudes of other persons or groups, and, by sharing their experience and history, are incorporated with them in a common cultural life" (Park/Burgess 1969 [1921]: 735). Assimilation wird von den Autoren somit als ein Prozess der Angleichung verstanden, der zu einer Eingliederung in das kulturelle Leben führt. Gleichzeitig bedeutet dies aber nicht, dass alle Merkmale der Herkunftskultur der Zugewanderten verschwinden. Vielmehr bezieht sich diese Angleichung auf die Sozialisation der Nachkommen der Zugewanderten (Faist 2000: 350). Park geht innerhalb seines Ansatzes nämlich davon aus, dass Assimilation ein langwieriger Prozess ist, der meist erst den Nachkommen der dritten Generation gelingt (Oswald 2007: 94/95). „Assimilation bedeutet letztendlich ein Verschmelzen der Kinder und Kindeskinder von Immigranten mit dem Kern der Mehrheitsgesellschaft““ (Faist 2000: 339).

Kritikpunkte an diesem Ansatz sind, dass Assimilation als ein geradliniger Prozess beschrieben wird, der nicht umkehrbar ist und in eine Richtung abläuft. Assimilationsprozesse, die nur teilweise stattfinden oder nicht gleichmäßig verlaufen, können somit mit Hilfe dieses Ansatzes 
nicht erklärt werden (Oswald 2007: 94/95). Darüber hinaus stellt sich nach dem Konzept des „Race-relation-cycle“ nicht die Frage, ob Assimilation überhaupt stattfindet, sondern nur, in welcher Geschwindigkeit diese vonstatten geht. Die Dauer der Assimilation ist dabei von der Unterschiedlichkeit der beiden Kulturen, also der Herkunftskultur und der Kultur der Aufnahmegesellschaft, abhängig (Hans 2016: 28).

Anknüpfend an die Idee der Assimilation folgten auf den „Race-relation-cycle“ einige Sequenzund Zyklenmodelle, in denen auch einzelne Bereiche der Assimilation näher differenziert wurden. Bereits innerhalb dieser Ansätze finden sich jedoch auch Modelle, die eher eine Art „partielle“ Assimilation beschreiben. So geht Eisenstadt (1951) in seinem Modell zwar von einem vollständigen Aufgehen der Zugewanderten in der Aufnahmegesellschaft aus, dafür muss aber auch die Sozialstruktur der Aufnahmegesellschaft so gestaltet sein, dass Zugewanderte alle Statuspositionen erreichen können. Darüber hinaus geht Eisenstadt auch davon aus, dass trotz des Aufgehens in der Gesellschaft ethnische Gruppen erhalten bleiben und große Unterschiede zwischen unterschiedlichen Zuwanderungsgruppen im Prozess der Assimilation bestehen (Oswald 2007: 102).

Eine klare Gegenposition zum Assimilationsmodell bezogen Glazer und Moynihan mit ihrer Arbeit „Beyond the Melting Pot“ (1963) und gelten damit als Begründer der Pluralismusdebatte (Aigner 2017: 27). Mit ihrer Gegenposition leiteten Glazer und Moynihan einen Paradigmenwechsel, hin $\mathrm{zu}$ mehr pluralistischen Theorien, ein. Im Zuge dieses Paradigmenwechsels kam es in der Migrations- und Integrationsforschung zur Weiterentwicklung von Konzepten zu ethnischem Pluralismus, Multikulturalismus und Ethnizität (Aigner 2017: 28/29). Glazer und Moynihan gehen davon aus, dass eine vollständige Assimilation nicht stattfinden kann. Vielmehr führt der Einfluss des Aufnahmelandes nur zu einer teilweisen Veränderung der Einwanderinnen und Einwanderer, wodurch etwas Neues und auch eine pluralistische Struktur in der Gesellschaft entsteht (Glazer/Moynihan 1970: 13/14). Denn die ethnischen Gruppen bleiben als einzelne Gruppen identifizierbar und ordnen sich auch selbst über Generationen „ihrer“ Gruppe zu. Hierzu führen die Autoren aus: „...they were recreated as something new, but still as identifiable groups. Concretely, persons think of themselves as members of that group, with that name; and most significantly, they are linked to other members of the group by new attributes that the original immigrants would never have recognized as identifying their group, but which nevertheless serve to mark them off, by more than simply name and association, in the third generation and even beyond" (Glazer/Moynihan 1970: 13). 
Als Reaktion auf die Arbeit von Glazer und Moynihan entstand Gordons „Assimilation in American Life“ (1964), worin Gordon mit seiner Assimilationstheorie deutliche Kritik am Konzept des kulturellen Pluralismus übte. Durchsetzen konnte sich Gordon gegen die Debatte nicht (Aigner 2017: 34). Dennoch entstand damit das differenzierteste Assimilationsmodell (Faist 2000: 351).

Gordon unterscheidet insgesamt sieben Bereiche der Assimilation. Diese umfassen eine kulturelle, strukturelle, heiratsmäßige, identifikative, einstellungsbezogene, verhaltensbezogene und zivilgesellschaftliche Ebene. Darüber hinaus geht Gordon davon aus, dass die Assimilation innerhalb der differenzierten Bereiche zu einem unterschiedlichen Grad stattfinden kann (Gordon 1964: 71). Wie auch Eisenstadt (1954), stellt Gordon in seiner Arbeit die Allgemeingültigkeit der Assimilation in Frage (Nobis/Strahle 2009: 120), indem er zunächst drei unterschiedliche theoretische Ausgänge von Integration diskutiert. Diese umfassen das Modell der einseitigen Assimilation an die Aufnahmegesellschaft, ein Modell gegenseitiger Assimilation von Zugewanderten und der Aufnahmegesellschaft („Melting Pot") sowie ein Modell, indem ethnische Unterschiede bestehen bleiben (,Salad Bowl““/"cultural pluralism“). Gordon sieht jedoch die klassische Annahme der einseitigen Assimilation an die „,core society“ im Falle der USA als wahrscheinlichste Form der Integration von Zugewanderten an, die sich gegen die anderen beiden Ausgänge durchsetzen wird (Vgl. Gordon 1964). Gleichzeitig stellt er aber auch fest, dass andere Ausgänge von Integration durchaus stattfinden können und spricht dadurch auch anderen Ausgängen von Integration Gültigkeit zu. Dazu führt er aus: „cultural pluralism was a fact in American life before it became a theory"(Gordon 1964: 135).

Neuere Ansätze zur Assimilation, wie beispielsweise die Ausarbeitungen zu Neoassimilation und segmentierter Assimilation, sehen die Ausgestaltung des Konzepts der Assimilation offener.

Alba und Nee (2003) diskutieren in ihrer Arbeit „Remaking the American Mainstream. Assimilation and Contemporary Immigration“ das Konzept der Assimilation vor dem Hintergrund aktueller Einwanderungsprozesse in den USA und stellen ihre neue, offenere Theorie der Neoassimilation vor. Im Rahmen dieses Ansatzes verstehen Alba und Nee Assimilation nicht als Anpassung, sondern als Abmilderung von Unterschieden, die auf Herkunft oder ethnischem Hintergrund beruhen. Herkunft und ethnischer Hintergrund verlieren im Rahmen ihres Konzepts ihre Relevanz als Differenzlinie. Die Autoren gehen davon aus, dass Assimilation kein universelles Ergebnis darstellt und auch nicht unvermeidlich stattfindet. Vielmehr können auch andere Wege zur Adaption führen (Alba/Nee 2003: 38). Assimilation 
stellt somit zwar einen verbreiteten, aber nicht den einzigen Weg dar (Hans 2016: 35/36). Darüber hinaus kann sich das Ausmaß der Assimilation von Gruppe zu Gruppe und auch innerhalb einer Gruppe unterscheiden (Alba/Nee 2003: 38). Das führen die Autoren darauf zurück, dass die einwandernden Personen eine höhere Diversität aufweisen, als es früher der Fall war. „The contemporary migration is more diverse than that of the past, in terms of capital immigrants bring, the nature of the communities they enter, and their race and legal status. What can be expected is that individuals and groups will acculturate at different rates and often selectively, by shedding some aspects of immigrant cultural practices while retaining others" (Alba/Nee 2003: 274). Assimilation ist darüber hinaus nicht als ein einzelner Mechanismus zu verstehen, sondern als Zusammenspiel unterschiedlicher Mechanismen auf unterschiedlichen Ebenen der Gesellschaft (Alba/Nee 2003: 39). Dieser Ansatz unterscheidet sich somit deutlich von der früheren Auslegung von Assimilation. Zum einen, weil der Begriff der Assimilation nicht mit der einseitigen Anpassung verbunden wird, sondern eher einen beidseitigen und intergenerationalen Prozess darstellt. Zum anderen zeigt sich eine Offenheit in der Ausgestaltung des Prozesses der Assimilation. Dieser Prozess kann sich von Gruppe zu Gruppe, aber auch innerhalb von Gruppen unterscheiden, während Assimilation in den früheren Sequenz- und Zyklenmodellen als unausweichlich und geradlinig dargestellt wurde. Nichtsdestotrotz machen die Autoren auch deutlich, dass der Prozess der Assimilation schon immer vorkam und dieser Prozess auch in der Zukunft stattfinden wird. Dazu schreiben die Autoren: „But the key conclusion for us is, that there will be some continuity in assimilation between past and future“(Alba/Nee 2003: 273/274).

Portes und Zhou (1993) beschreiben in ihrer Arbeit „The New Second Generation: Segmented Assimilation and it's Variants“ eine weitere offenere Ausgestaltung von Assimilation, die als segmentierte Assimilation bezeichnet wird. Im Rahmen dieses Ansatzes existieren drei mögliche Pfade der Assimilation. Der erste ist die klassische Assimilation an die weiße Mittelschicht. Weitere Möglichkeiten sind die Assimilation an die Unterschicht (downward assimilation) oder ein schneller wirtschaftlicher Aufstieg unter Wahrung der Werte der Herkunftsgesellschaft und enger Solidarität mit ihr (selective acculturation) (Portes/Zhou 1993: 82). Dazu führen Portes und Zhou aus: „In other words the question is into what sector of American society a particular immigrant group assimilates. Instead of a relatively uniform mainstream whose mores and prejudices dictate a common path of integration, we observe today several distinct forms of adaption“ (Port/Zhou 1993: 82). In welchen Sektor sich eine Einwanderungsgruppe assimiliert, ist dabei von individuellen und familiären sowie von kontextuellen Faktoren des Einwanderungslandes abhängig (Portes/Zhou 1993: 82/83). 
Die Ausführungen zu den unterschiedlichen Assimilationsmodellen zeigen, dass sich diese über die Jahre stetig weiterentwickelt haben. Zum einen führten diese Entwicklungen zu einer offeneren Auslegung des Assimilationsbegriffs und zum anderen auch dazu, dass Assimilation nicht mehr als einziger Ausgang von Integration diskutiert wird. Die ersten sogenannten „Straight-line-Theorien“ zeigen ein Verständnis von Assimilation, das diese als geradlinigen und unausweichlichen Prozess darstellt. Genau dieses Verständnis geriet dabei immer wieder in Kritik, da eine Assimilation, die irreversibel in eine Richtung abläuft, partielle und auch eventuell ungleichmäßig stattfindende Prozesse der Assimilation nicht erklären oder beschreiben kann (Oswald 2007: 95). Darüber hinaus werden diese klassischen Assimilationsansätze als ethnozentrisch und normativ bezeichnet (Hans 2016: 34). Und auch die Heterogenität von Einwanderungsgruppen fand in diesen Ansätzen keine Beachtung (Nobis/Strahle 2009: 122). Die vorgestellten neueren Assimilationsmodelle gehen auf diese Kritik ein und beschreiben Assimilation als einen offeneren Prozess. Zum einen stellt Assimilation nicht den einzigen möglichen Ausgang von Integration dar, sondern wird als ein verbreiteter Weg unter mehreren Möglichkeiten dargestellt. Zum anderen spielt auch die Heterogenität von Einwanderungsgruppen und innerhalb der Gruppen selbst eine Rolle (Vgl. Alba/Nee 2003). Portes und Zhou (1997) diskutieren zusätzlich die Möglichkeit der Assimilation an bestimmte Segmente einer Gesellschaft.

Auch der Begriff der Assimilation an sich hat über die Zeit eine neuere und offenere Bedeutung erhalten. So wurde der Begriff lange Zeit als Anpassung der Zuwanderungsgruppen an die Mehrheitsgesellschaft verstanden. In den vorgestellten neueren Modellen umfasst die Ausgestaltung dieses Begriffs hingegen eine Abmilderung von Unterschieden, die auf Herkunft oder ethnischem Hintergrund beruhen, und durch beidseitige Prozesse stattfinden kann. Nichtsdestotrotz ,führen Begriffe wie Assimilation die historische Last fragwürdiger ideologischer Annahmen mit sich“ (Faist 2000: 352), da ihre ursprüngliche Auslegung immer noch präsent ist.

Neben neueren Assimilationsmodellen gibt es auch Ansätze, die die aus der Arbeit von Glazer und Moynihan entstandene Idee des Pluralismus aufgreifen und somit Alternativen zum Assimilationsansatz bieten. Beispiele, die hier zu nennen sind, sind der ethnische Pluralismus und der Multikulturalismus. Neben den Assimilationsmodellen stellen diese Ansätze die bekanntesten Ansätze in der Integrationsforschung dar (Faist 2000: 348).

Der ethnische Pluralismus stellt das Konzept der Assimilation in Frage und bildet das Gegenkonzept zum „Melting Pot“ (Aigner 2016: 28). Grundidee des ethnischen Pluralismus ist die Wertschätzung von Diversität. Darüber hinaus sollen unterschiedliche Kulturen als 
gleichwertig anerkannt werden (Hans 2016: 39). Dies bedeutet jedoch nicht, dass Assimilationstheorien diese Punkte per se bestreiten.

Der Multikulturalismus baut auf den Gedanken des ethnischen Pluralismus auf und erweitert diesen (Aigner 2017: 28). Teilweise werden die Begriffe aber auch synonym verwendet (Nobis/Strahle 2009: 122). Diese pluralistischen Integrationskonzepte gehen dabei davon aus, dass in modernen Gesellschaften eine Diversität und Vielzahl von eigenständigen, ethnischen Subgruppen nebeneinander existieren, die dabei mehr oder weniger isoliert voneinander sind. In einer normativen Perspektive geht es dann darum, ob diese Eigenständigkeit auch gewollt und akzeptiert ist. Im Gegensatz zum Anpassungsgedanken der Assimilation geht es in diesen Ansätzen somit überwiegend um die Anerkennung von Vielfalt und Differenz durch die Aufnahmegesellschaft (Nobis/Strahle 2009: 122). Somit enthalten diese pluralistischen Konzepte zwei Lesarten. Die erste Lesart bezieht sich auf die kulturelle Eigenständigkeit ethnischer Gruppen, während die zweite Lesart das Prinzip der wechselseitigen Anerkennung betont (Nobis/Strahle 2009: 123).

Ein Problem an diesen Ansätzen ist, dass sie schnell in einen normativen Diskus übergehen, in dem wünschenswerte oder geeignete Ausgänge von Integration diskutiert werden (Nobis/Strahle 2009: 122). Hierzu schreibt Faist (2000): „Die dahinterstehende normative Grundlegung besagt, daß Integration in Immigrationsländern am besten dadurch erreicht werden kann, wenn jede ethnische bzw. kulturelle Gruppe ihre eigene $>$ Nationalität $<$ leben kann. Aus der Sicht des Ethnischen Pluralismus gibt es keinen Widerspruch zwischen Integration und kultureller Vielfalt. Denn auch kulturelle Autonomie der Immigrantengruppe ist eine Form der Adaption im Immigrationsland“(Faist 2000: 353). Jedoch werden im Rahmen der pluralistischen Konzepte keine Erklärungsansätze diskutiert. Es bleibt somit unklar, wie ethnische Pluralität erreicht werden kann und auch das empirische Vorkommen der Assimilation wird im Rahmen der Ansätze oft ausgeblendet (Hans 2016: 40). Darüber hinaus wird auch diskutiert, ob diese pluralistischen Ansätze nicht eher in einer Ausbildung von Parallelgesellschaften enden (Nobis/Strahle 2009: 125).

Die Ausführungen zur Debatte um Assimilation und Pluralismus zeigen, dass beide Konzepte ihre Kritikpunkte haben und dass im Rahmen beider Konzepte immer noch eine Entwicklung stattfindet. Auffällig ist hierbei, dass die Ansätze dabei zwar eine offenere Ausgestaltung erhalten, im Grunde bleibt die dabei mitschwingende Diskussion um Assimilation oder Pluralismus aber erhalten. Was sich über die Zeit verändert, ist lediglich die Ausgestaltung der Konzepte. Und obwohl auch darüber diskutiert wird, dass die Ausgänge von Integration noch vielfältigere Formen annehmen können, stellen diese beiden Konzepte die am häufigsten 
diskutierten Ausgänge dar. Gans (1997) schreibt hierzu: „,What is in reality a range of adaptions is sometimes being turned into a dichotomy“ (Gans 1997: 875).

Darüber hinaus fällt auch auf, dass die Grenzen zwischen normativ wünschenswerten Ergebnissen von Integration und der empirischen Wirklichkeit oft verschwimmen. So wird Assimilation oft als empirische Tatsache dargestellt, gleichzeitig aber auch angeführt, dass Integration nur durch Assimilation stattfinden kann, was eher eine normative Prägung aufweist. Außerdem bleibt bezüglich der Assimilation zwischen Assimilation als Prozess und als Zielzustand zu unterscheiden. Dennoch stellen die beiden Konzepte für die vorliegende Arbeit wichtige Ansätze in Bezug auf die Untersuchung wünschenswerter Ausgänge von Integration für Personen außerhalb des wissenschaftlichen Diskurses dar. Zum einen, weil die Konzepte in Bezug auf ihre „Zielvorstellung“ - Anpassung/Abmilderung von Unterschieden oder Existenz eigenständiger Subgruppen - gut voneinander abgrenzbare Konstrukte bieten. Zum anderen dürften gerade diese beiden Konzepte auch außerhalb des wissenschaftlichen Diskurses bekannt sein. Da Assimilation und Pluralismus jedoch, wie bereits erwähnt, nicht die einzigen Ausgänge von Integration darstellen, werden im nächsten Unterkapitel auch noch die Ausgänge von Integration nach Hartmut Esser (2001) vorgestellt, die für die Operationalisierung später als Ergänzung herangezogen werden sollen.

\subsubsection{Mehrfachintegration, Assimilation, Marginalität und Segmentation nach} Hartmut Esser

Esser (2001) beschreibt in seinem Ansatz insgesamt vier mögliche Ausgänge von Integration, die sich durch den Bezug zur Aufnahme- und Herkunftsgesellschaft ergeben. Diese vier möglichen Ausgänge gehen auf Berry $(1990,1997)$ zurück. Innerhalb seiner Arbeit diskutiert Berry die Kategorien kulturelle Erhaltung und Kontakt/Teilnahme in Bezug auf die Akkulturation von Migrantinnen und Migranten. Die kulturelle Erhaltung umfasst dabei die Aufrechterhaltung der kulturellen Identität, während Kontakt/Teilnahme das Engagement in Gruppen bzw. die Abgrenzung von Anderen beinhaltet. Werden diese Kategorien zusammen herangezogen, ergeben sich vier mögliche Ausgänge (Berry 1997: 9/10).

Den Ausführungen liegt dabei der Rational-Choice-Ansatz zugrunde. Die Integration in die Aufnahmegesellschaft, bzw. ob diese überhaupt stattfindet, ist dabei vorrangig von der Investitionsbereitschaft der Migrantinnen und Migranten abhängig. Diese Investitionsbereitschaft unterliegt der persönlichen Motivation sowie einer Abwägung von Kosten und Nutzen. Beispielsweise werden Migrantinnen und Migranten, die nur eine kurze 
Aufenthaltsdauer im Land planen, keine Ressourcen in Form von Zeit oder Geld in das Erlernen der Sprache investieren, da der Nutzen der Sprache bei kurzer Aufenthaltsdauer im Land nicht im Verhältnis zu den Kosten steht. Neben der geplanten Aufenthaltsdauer spielen in Bezug auf die Investitionsbereitschaft auch das Alter und die Bildung der Migrantinnen und Migranten eine wichtige Rolle (Hans 2016: 31). Somit ist in Essers Ansatz der Ausgang der Integration von weiteren Faktoren abhängig und unterliegt einem rationalen Entscheidungsprozess der Personen.

Nach Esser bestehen die möglichen Ausgänge von Integration aus Mehrfachintegration, Assimilation, Marginalität und Segmentation. Diese Ausgänge sind möglich, da sich die Sozialintegration der Person auf drei unterschiedliche gesellschaftliche Systeme beziehen kann. Diese sind das Herkunftsland, das Aufnahmeland und auch die vorhandene ethnische Gemeinde im Aufnahmeland. Die Ausgänge der Sozialintegration unterscheiden sich somit je nachdem, in welche Kontexte die Akteurin oder der Akteur integriert ist. Tabelle 3 zeigt die möglichen Ausgänge der Sozialintegration sowie die gesellschaftlichen Systeme, in denen die Akteurin oder der Akteur im Rahmen des jeweiligen Ausgangs integriert ist (Esser 2001: 19).

Tabelle 3: Typen der (Sozial-) Integration von Migrantinnen und Migranten

\section{Sozialintegration in die Herkunftsgesellschaft}

Quelle: Esser 2001, S. 19.

\section{Sozialintegration in die Aufnahmegesellschaft}

\begin{tabular}{l|ll} 
& Ja & Nein \\
\hline Ja & Mehrfachintegration & Segmentation \\
Nein & Assimilation & Marginalität
\end{tabular}

Im Falle der Mehrfachintegration kommt es zu einer Sozialintegration in beide Gesellschaften, also sowohl in die Herkunfts- als auch in die Aufnahmegesellschaft. Ist die Person nur in die Herkunftsgesellschaft oder ethnische Gemeinde integriert, spricht man von Segmentation. Im Gegensatz dazu wird eine ausschließliche Integration in die Aufnahmegesellschaft als Assimilation bezeichnet. Ist die Person in keines der gesellschaftlichen Systeme integriert, wird der Begriff der Marginalität verwendet (Esser 2001: 19).

Fälle von Marginalität kommen gerade in der ersten Generation von Migrantinnen und Migranten häufiger vor. Hier wird beispielsweise keine der beiden Sprachen wirklich beherrscht, in beiden Gesellschaften keine akzeptable Position eingenommen, es sind keine sozialen Beziehungen zu Personen aus einer der beiden Gesellschaften vorhanden und/oder es findet keine Identifizierung mit der Herkunfts- oder Aufnahmegesellschaft statt. Esser schreibt 
hierzu: „Der marginale Akteur ist ein ausgestoßener, einsamer und heimatloser Fremder, wohin auch immer er geht“" (Esser 2001: 20).

Ein weiterer von Esser beschriebener Ausgang ist die Segmentation. Diese umfasst „die Sozialintegration in ein binnenethnisches Milieu und die gleichzeitige Exklusion aus den Sphären und den Milieus der Aufnahmegesellschaft"“. Es bilden sich beispielsweise ethnische Gemeinden oder „Ghettos“ heraus. Diese bieten eine Art Alternative zur Lebensgestaltung und können so zur Segmentation kompletter ethnischer Gemeinden führen (Esser 2001: 20).

Nach Esser ist die Mehrfachintegration ein wünschenswerter, aber sehr seltener Fall. Dieser Fall der Sozialintegration zeigt sich beispielsweise durch die Beherrschung beider Sprachen oder eine mehrfache Identifikation (Esser 2001: 20). Dieser Ausgang der Sozialintegration tritt selten auf, da den Akteurinnen und Akteuren bestimmte Lern- und Interaktionsaktivitäten abverlangt werden. Gelegenheiten, diese Lern- und Interaktionsaktivitäten wahrzunehmen, bleiben den Akteurinnen und Akteuren jedoch in der Herkunfts- und/oder Aufnahmegesellschaft oft verwehrt. Esser schreibt hierzu „Dieser Typ der ,multikulturellen“ Sozialintegration käme allenfalls für Diplomatenkinder oder für Akademiker in Frage, in deren Familien sich etwa die Eltern mit ihren Kindern in mehreren Sprachen unterhalten können und bei denen die verschiedenen Kulturen tatsächlich auch im Alltag zu Hause sind“" (Esser 2001: 21). Darüber hinaus fügt Esser an, dass aufgrund von sozialpsychologischen Prozessen und der Neigung, die Welt in Ingroup- und Outgroup-Schemata zu betrachten, in den meisten Fällen eine vorrangige Integration in das eine oder andere gesellschaftliche System vorliegt (Esser 2001: 21).

Aus diesem Grund ist für Esser die Integration in die Aufnahmegesellschaft nur in Form der Assimilation möglich. Für Esser ist die Assimilation somit ein spezieller Fall der Sozialintegration und kein eigenständiges Konzept (Hans 2016: 33). Unter Assimilation wird im Allgemeinen ,die ,Angleichung‘ der verschiedenen Gruppen in bestimmten Eigenschaften verstanden, etwa im Sprachverhalten oder in der Einnahme beruflicher Positionen“" (Esser 2001: 21). Diese Ansätze gehen von einem notwendigen Konsens innerhalb der Gesellschaft und einer damit einhergehenden „Homogenität“ der Gesellschaft aus (Schulte 2000: 219). Ziel der Integration ist hier somit eine Anpassung der Migrantinnen und Migranten, beispielsweise durch die Übernahme von Werten und Praktiken, welche zu einer möglichst hohen Homogenität der Gesellschaft führt (Han 2010: 65). Der Begriff der Assimilation ist sowohl normativ als auch politisch stark belastet ist. So wird dieser teilweise als „die völlige Identifikation der Angehörigen von Minderheiten mit der Kultur der dominanten Mehrheit 
verstanden. Dabei wird unterstellt, dass die Angehörigen der Minderheiten eine über die externe und interne Akkulturation hinausgehende neue Identität und Loyalität annehmen, die sich auf die dominante Kultur stützen“ (Han 2010: 295). Von der Verwendung des Begriffs in dieser Form oder als Angleichung in Form von der Herstellung von Homogenität sieht Esser ab und beschreibt Assimilation als das Ausbleiben von ,systematischen Unterschieden in der Verteilung gewisser Eigenschaften und Ressourcen über die verschiedenen Gruppen einer Gesellschaft" hinweg (Esser 2001: 21). Da selbst die einheimische Bevölkerung nicht homogen ist, kann es nach Esser nur darum gehen, Verteilungen anzugleichen und dass somit keine systematischen Unterschiede in beispielsweise Eigenschaften oder Ressourcen vorliegen. Dieser Prozess muss keineswegs einseitig ablaufen, sondern die Verteilungen können sich über verschiedene Gruppen hinweg angleichen. Der Ansatz unterscheidet sich somit von der früheren Vorstellung von Assimilation im Rahmen derer davon ausgegangen wurde, dass es sich um einen einseitigen Prozess handelt. Gleichzeitig führt Esser jedoch auch an, dass dies nur in seltenen Fällen und in niedrigschwelligen Bereichen wie beispielweise den Essgewohnheiten oder dem Freizeitverhalten vorkommt (Esser 2001: 21). Darüber hinaus ist die Assimilation auch an Voraussetzungen geknüpft und wird nur vollzogen, sofern die Akteurinnen und Akteure die Assimilation in die Gesellschaft des Aufnahmelandes beabsichtigen, dies auch möglich ist und es keine Alternative in Form der Rückkehr in das Heimatland oder die Integration in eine ethnische Gemeinde gibt (Esser 2001: 25). Wie im Fall der Sozialintegration unterscheidet Esser auch bei der Assimilation die vier Dimensionen kulturelle, strukturelle, soziale und identifikative Assimilation, welche mit den im Rahmen der Integrationsdimensionen beschriebenen Dimensionen übereinstimmen (Esser 2001: 22) ${ }^{13}$. Die enge Beziehung von Assimilation und Sozialintegration rührt bei Esser daher, dass er lediglich die Assimilation als vollzogene Sozialintegration versteht. Denn „Die Sozialintegration etwa nur in die ethnische Gemeinde, die Beibehaltung der Sozialintegration in das Herkunftsland oder gar die Marginalisierung der Personen und Gruppen zwischen allen Stühlen, kann mit der sozialen ,Integration“ der Migranten ja sicher nicht gemeint sein. Daher kann man die vollzogene Sozialintegration von Migranten in die Aufnahmegesellschaft realistischerweise, bis auf die wenigen Ausnahmen von Diplomaten, Akademikern oder sonstigen Kosmopoliten, wohl kaum anders als denn ,Assimilation“ verstehen“ (Esser 2001: 23).

\footnotetext{
${ }^{13}$ Siehe Kapitel 4.2.3 Hartmut Esser für eine ausführliche Beschreibung der unterschiedlichen Dimensionen.
} 


\subsubsection{Schlussfolgerungen zu Ergebnissen von Integration für die eigene Arbeit}

Im Rahmen der vorliegenden Arbeit soll, in Bezug auf die Ausgänge von Integration, die Frage beantwortet werden, welche Ergebnisse von Integration von den Akteurinnen und Akteuren für wünschenswert gehalten werden, bzw. welchen Ausgängen von Integration die Akteurinnen und Akteure am meisten zustimmen.

Die vorangegangenen Unterkapitel dienen der späteren Operationalisierung zur Beantwortung der Frage zu Einstellungen zu Integrationskonzepten. Für die Beantwortung dieser Frage lässt sich aus den vorangegangenen Ausführungen festhalten, dass innerhalb der Konzepte der Assimilation und des Pluralismus über die Jahre Entwicklungen stattgefunden haben, die zu einer offeneren Ausgestaltung der Konzepte geführt haben. Dennoch existiert immer noch eine grundlegende Diskussion über Assimilation oder Pluralismus als Ausgang von Integration und die beiden Konzepte bilden in Bezug auf die vorliegende Arbeit wichtige Ansätze zur Untersuchung wünschenswerter Ausgänge von Integration. Assimilative und pluralistische Ansichten bilden darüber hinaus zunächst gut voneinander abgrenzbare Konstrukte und dürften auch, zumindest in ihrer Bedeutung, außerhalb des wissenschaftlichen Diskurses bekannt sein.

Darüber hinaus wurde auch ersichtlich, dass die Debatte um Assimilation und Pluralismus zwar einen großen Raum einnimmt, dennoch stellen diese Konzepte nicht die einzigen Ausgänge von Integration dar. Um die Ausgänge von Integration zu erweitern, wurde außerdem das Konzept von Hartmut Esser (2001) vorgestellt, der insgesamt vier Ausgänge von Integration beschreibt. Diese vier Ausgänge umfassen die Marginalität, Assimilation, Segmentation und Mehrfachintegration. Die Marginalität als fehlende Einbindung in einen der gesellschaftlichen Kontexte, erhält im weiteren Verlauf der Arbeit keine Bedeutung, da dieser Ausgang nicht als wünschenswert eingeschätzt wird. Den Begriff der Assimilation verwendet Esser zwar in einer offeneren Weise als die klassischen Assimilationsmodelle, dennoch stellt die Assimilation als Sozialintegration in die Aufnahmegesellschaft kein klar abgrenzbares Konstrukt zum generellen Begriff der Assimilation dar. Innerhalb des Ansatzes von Esser sind für die vorliegende Arbeit vor allem die Konzepte der Mehrfachintegration und der Segmentation, als Ergänzung zu den Konzepten Assimilation und Pluralismus, interessant und sollen im Rahmen der Umfrage von den Befragten bewertet werden. Die Mehrfachintegration beschreibt einen Zustand, in dem die Personen sowohl in die Aufnahmegesellschaft als auch in die Herkunftsgesellschaft integriert sind. Dieser Ausgang lässt sich dem Feld des Pluralismus zuordnen, jedoch in einer Ausgestaltung, die nicht die komplette Beibehaltung der Kultur des Herkunftslandes beinhaltet, und stellt somit ein weiteres gut abgrenzbares Konstrukt zur 
Assimilation dar. Die Segmentation beschreibt einen Zustand der Sozialintegration, in dem die Personen lediglich in die Herkunftsgesellschaft integriert sind, was für manche der Befragten durchaus ein wünschenswerter Zustand sein könnte, in dem nämlich eine Abgrenzung zur Gesellschaft des Aufnahmelandes stattfindet. Im Gegensatz zum Pluralismus bleiben die Gruppen dabei jedoch isoliert unter sich, wodurch man von einer gewissen Koexistenz der Gruppen sprechen kann. Pluralistische Konzepte unterstützen jedoch darüber hinaus die Akzeptanz von Diversität und die wechselseitige Anerkennung der Gruppen. Dadurch können auch Verbindungen zwischen den unterschiedlichen Gruppen bestehen. Aus diesem Grund soll auch die Segmentation als Ausgang von Integration in die Befragung aufgenommen werden.

Die nachfolgende Tabelle 4 fasst abschließend die Ausgänge von Integration zusammen, die im Rahmen dieser Arbeit betrachtet werden sollen.

Tabelle 4: In dieser Arbeit betrachtete Ausgänge von Integration

Ausgang von Integration

\begin{tabular}{l|l}
\hline Assimilation & $\begin{array}{l}\text { Es findet eine Anpassung an die } \\
\text { Aufnahmegesellschaft statt. }\end{array}$ \\
\hline Pluralismus & $\begin{array}{l}\text { Die Personen behalten ihre Kultur bei. Es } \\
\text { herrscht eine Anerkennung von Diversität } \\
\text { sowie eine wechselseitige Anerkennung der } \\
\text { Gruppen. }\end{array}$ \\
\hline Mehrfachintegration & $\begin{array}{l}\text { Die Personen sind sowohl in die } \\
\text { Aufnahmegesellschaft als auch in die }\end{array}$ \\
& Herkunftsgesellschaft integriert. Dieser \\
& Ausgang lässt sich dem Feld des \\
Pluralismus zuordnen, jedoch in einer \\
Ausgestaltung, die nicht die komplette \\
Beibehaltung der Kultur des \\
\\
Herkunftslandes beinhaltet.
\end{tabular}

\section{Bedeutung}

Es findet eine Anpassung an die Die Personen behalten ihre Kultur bei. Es herrscht eine Anerkennung von Diversität Gruppen.

Die Personen sind sowohl in die Herkunftsgesellschaft integriert. Dieser Ausgang lässt sich dem Feld des Pluralismus zuordnen, jedoch in einer Ausgestaltung, die nicht die komplette Beibehaltung der Kultur des Die Personen sind lediglich in die Herknftsgesellschaft integriert.

Quelle: Eigene Darstellung. 


\subsection{Dimensionen von Integration}

Neben den Ausgängen von Integration werden in der vorliegenden Arbeit auch Dimensionen und Aspekte von Integration behandelt. In diesem Unterkapitel werden daher Konzeptionen von Integrationsdimensionen unterschiedlicher Autorinnen und Autoren dargestellt und diskutiert. Dabei soll jedoch nicht jeweils der gesamte theoretische Ansatz der Arbeiten dargestellt werden, sondern nur Konzepte, die sich innerhalb dieser Theorien mit dem Begriff der Integration und unterschiedlichen Dimensionen auseinandersetzen. Diese Diskussion dient der Ableitung von möglichst breit gefächerten Integrationsdimensionen für die spätere Befragung. Zunächst soll hierbei auf theoretische Konzepte von Integration innerhalb der Migrationstheorie von Shmuel N. Eisenstadt (1951/1952) eingegangen werden, da Eisenstadt mit seiner Migrationstheorie zu den Anfängen der Migrationsforschung im englischsprachigen Raum gehört. Aus dem deutschsprachigen Raum werden Theorien von Hans-Joachim Hoffmann-Nowotny (1973), Hartmut Esser (2001), Friedrich Heckmann et al. (2000) sowie Reimund Anhut und Wilhelm Heitmeyer (2009) skizziert. Hoffmann-Nowotny und Esser gehören zu den bekanntesten Autoren im Bereich migrationssoziologischer Fragen im deutschsprachigen Raum und beschreiben ein breit differenziertes Bild von Integration. Heckmann orientiert sich an den Dimensionen von Esser und erweitert dabei die Integrationsdimensionen um weitere Aspekte. Darüber hinaus soll auch auf den Bielefelder Desintegrationsansatz nach Anhut und Heitmeyer eingegangen werden, da dieser einen anderen Blickwinkel auf Integration, mit dem Ziel der Anerkennung, verfolgt.

\subsubsection{Shmuel N.Eisenstadt}

Shmuel N. Eisenstadt beschäftigt sich im Rahmen seiner Migrationstheorie mit der „Absorption“ von Migrantinnen und Migranten in Israel (Eisenstadt 1952a: 223). Seine Migrationstheorie enthält insgesamt drei Phasen der Migration. Diese umfassen die Motivbildung zur Migration, den Vorgang der Migration und den Prozess der Eingliederung in die Aufnahmegesellschaft ${ }^{14}$ (Han 2010: 43/44).

Insgesamt beschreibt Eisenstadt den Prozess der Eingliederung in die Aufnahmegesellschaft als „Absorption“ (Han 2010: 44), welche einen Prozess der Ausweitung von Partizipation der Migrantinnen und Migranten beschreibt (Eisenstadt 1952b: 226). Diese ist wiederrum in drei Teilprozesse untergliedert, die für seine spätere Thematisierung von Integration von Bedeutung

\footnotetext{
${ }^{14}$ Im Folgenden wird nur auf die dritte Phase der Migrationstheorie näher eingegangen, da diese Phase verschiedene Dimensionen von Integration thematisiert.
} 
sind (Han 2010: 44). So unterscheidet Eisenstadt die Teilprozesse Institutionalisierung der Rollenerwartungen und Verhaltensweisen im Alltag, Anpassung der Migrantinnen und Migranten an die Anforderungen der Aufnahmegesellschaft sowie Eindringen der Migrantinnen und Migranten in die institutionellen Sphären der Aufnahmegesellschaft und Verschmelzung. Die Institutionalisierung der Rollenerwartungen und Verhaltensweisen im Alltag bezieht sich auf das Erlernen von Fähigkeiten, die für die Bewältigung bestimmter Rollenerwartungen der Aufnahmegesellschaft von Bedeutung sind (Eisenstadt 1952b: 373). Diese umfassen Sprache, Werte, Normen, Gewohnheiten und Erwartungen der aufnehmenden Gesellschaft (Han 2010: 45). Dadurch kommt es auch zu einer Transformation der Primärgruppen und sozialen Beziehungen der Migrantinnen und Migranten (Eisenstadt 1952b: 373). Diese Transformation der Primärgruppen und sozialen Beziehungen ist nach Eisenstadt wichtig für den zweiten Teilprozess: Die Anpassung der Migrantinnen und Migranten an die Anforderungen der Aufnahmegesellschaft. Für die erfolgreiche Absorption in die Aufnahmegesellschaft, muss es zu einer Expansion der sozialen Interaktionen und Partizipation kommen. Diese muss dabei über primäre Gruppenbeziehungen hinausgehen (Eisenstadt 1951: 223). Jedoch hängt diese Anpassung nicht nur von den Migrantinnen und Migranten ab, sondern auch von der Bereitschaft der Aufnahmegesellschaft (Han 2010: 45). Der dritte Teilprozess der Eingliederung in die Aufnahmegesellschaft beschreibt das Eindringen der Migrantinnen und Migranten in die institutionellen Sphären der Aufnahmegesellschaft und die Verschmelzung. Im Rahmen dieses Teilprozesses verlieren die Migrantinnen und Migranten ,ihre separate ethnische Gruppenexistenz und -identität“ (Han 2010: 46). Dabei ist es auch von Bedeutung, dass Migrantinnen und Migranten in der Struktur des Aufnahmelandes gleichmäßig über unterschiedliche Ebenen verteilt sind und sich nicht beispielsweise in unteren Schichten der Gesellschaft konzentrieren (Hoffmann-Nowotny 1973: 171).

Neben diesen im Rahmen der Eingliederung ablaufenden Prozessen, beschreibt Eisenstadt auch unterschiedliche Dimensionen von Integration, die in unterschiedlichen Strukturbereichen der Gesellschaft im Rahmen der beschriebenen Teilprozesse stattfinden (Han 2010: 309). Insgesamt unterscheidet Eisenstadt adaptive, instrumentale, solidarische sowie kulturelle Integration.

Unter adaptiver Integration versteht Eisenstadt das Erlernen von Basisrollen in den Hauptinstitutionen wie beispielsweise Familie, Erziehung, Wirtschaft und Politik. Neben dem Erlernen von Rollen gehören zur adaptiven Integration auch der Aufbau sozialer Kontakte mit 
Einheimischen sowie eine positive Identifikation mit den Werten und Strukturen der aufnehmenden Gesellschaft (Han 2010: 309).

Mit dem Begriff der instrumentalen Integration bezeichnet Eisenstadt die Übernahme von Rollen im Wirtschaftsleben der Aufnahmegesellschaft. Diese dienen dazu, persönliche Ressourcen einzusetzen sowie Grundbedürfnisse zu stillen und bedeuten deshalb nicht, dass Rollen und Wertvorstellungen der Aufnahmegesellschaft übernommen werden (Han 2010: $310)$.

Die Identifikation und Solidarisierung mit Wertvorstellungen finden erst im Rahmen der solidarischen Integration statt. Im Rahmen dieser Dimension kommt es auch zur Entwicklung eines Zugehörigkeitsgefühls und aktiver Partizipation (Eisenstadt 1952b: 374).

Die letzte Dimension stellt bei Eisenstadt die kulturelle Integration dar. Hier werden emotionale Ausdrucksformen und Symbole der Aufnahmegesellschaft übernommen und bringen so Wertvorstellungen im Alltag zum Ausdruck (Han 2010: 311).

Eisenstadt geht nicht von einem linearen Verlauf der Integration aus, sondern dass nicht alle Integrationsprozesse durchlaufen werden müssen und es dadurch auch zu einer Pluralisierung der Aufnahmegesellschaft kommt (Han 2010: 311).

\subsubsection{Hoffmann-Nowotny}

Hoffmann-Nowotny $(1973 ; 1990)$ beschäftigt sich in seiner Arbeit mit der Soziologie des Fremdarbeiterproblems. Dazu verwendet er Daten aus einer Umfrage unter in Zürich lebenden Personen aus der Schweiz und aus Italien. Im Rahmen seiner Studie diskutiert er auch die Begriffe Assimilation und Integration im Rückgriff auf die Konzeption von Eisenstadt. Im Rahmen der Diskussion der Begriffe stellt Hoffmann-Nowotny fest: „Als offizielle und von der Bevölkerung weitgehend unterstütze Politik der Schweiz gilt also, daß nur voll assimilierte Einwanderer auch den letzten Schritt zur vollständigen Integration, die Aufnahme in das Bürgerrecht, tun können“ (Hoffmann-Nowotny 1973: 175).

Nach Hoffmann-Nowotny besteht die Gesellschaft aus zwei Teilsystemen - Struktur und Kultur. Dabei beschreibt Kultur die Symbolstruktur und Gesellschaft die Positionsstruktur. Durch die Aufteilung in diese Teilsysteme findet auch die Unterscheidung zwischen Integration und Assimilation statt. Integration bedeutet dabei Partizipation an der Struktur der Gesellschaft und Assimilation Partizipation an der Kultur der Gesellschaft. Zur Struktur der Gesellschaft 
gehören dabei Aspekte wie die rechtliche Stellung, Wohnen, Bildung, berufliche Stellung, Einkommen und soziale Interaktion. Die Kultur der Gesellschaft umfasst hingegen die Aspekte Sprache, Normen und Wertorientierungen. Integration bezieht sich somit, nach Auffassung des Autors, auf die Verteilung/den Platz der Migrantinnen und Migranten innerhalb unterschiedlicher Subsysteme einer Gesellschaft (berufliche Stellung, Einkommen, Bildung, etc.) (Hoffmann-Nowotny 1973: S. 171 ff.). Innerhalb dieser Unterscheidung ist auch zu beachten, dass Assimilation bei Hoffmann-Nowotny auf die Motivation der Migrantinnen und Migranten abzielt, wohingegen Integration von den Opportunitäten im Aufnahmeland abhängt (Hoffmann-Nowotny 1990: 17).

Hoffmann-Nowotny kritisiert darüber hinaus die Konzentration vieler Autorinnen und Autoren auf eine kulturelle Sphäre (Hoffmann-Nowotny 1973: 172). Zwischen Integration und Assimilation ergeben sich zwar Interdependenzen, jedoch determiniert die Integration die Assimilation in einem stärkeren Maße (Hoffmann-Nowotny 1973: S. 173). Das bedeutet, dass zunächst von Bedeutung ist, ob die Gesellschaft „die zentralen Statuslinien für die Migranten öffnet oder sie weitgehend geschlossen hält“ (Hoffmann-Nowotny 1973: 172). „Der Integration der Einwanderer und ihren Determinanten kommt nicht nur besondere Bedeutung zu, weil geringe Integration hohe anomische Spannungen erzeugt. Der Faktor ,Integration“ ist auch deshalb bedeutsam, weil die Assimilation in erster Linie eine Funktion der Integration ist. Einwanderer, die nicht integriert sind, assimilieren sich auch nicht. Selbst bei längerer Aufenthaltsdauer nehmen Assimilation und Assimilationsbereitschaft nicht zu, wenn der Einwanderer strukturell marginal bleibt" (Hoffmann-Nowotny 1973: 266). Die Begrifflichkeiten Integration und Assimilation beziehen sich in diesem Fall nicht nur auf Migrantinnen und Migranten, sondern es können auch andere Gruppen innerhalb der Gesellschaft, wie beispielsweise Frauen oder Männer, fokussiert werden (Hoffmann-Nowotny 1973: 172).

\subsubsection{Hartmut Esser}

Esser (2001) verdeutlicht in seinem Beitrag zunächst die allgemeine Bedeutung von Integration. Demnach versteht man unter Integration einen "relativ gleichgewichtigen Zusammenhalt der Teile eines Ganzen und dessen Abgrenzung gegen eine unspezifische Umgebung. Erst durch diesen Zusammenhalt wird ein System für seine Umwelt als System erkennbar. Aufgrund der Abhängigkeit der Teile des Systems untereinander, haben diese wiederrum Auswirkungen auf das System insgesamt“ (Esser 2001: 1). Nach Esser (2001) ist 
beim Begriff der Integration zwischen System- und Sozialintegration zu unterscheiden. Diese Unterscheidung wurde bereits von Lockwood (1964) eingeführt. Dabei bezieht sich die Systemintegration auf die Integration „des Systems einer Gesellschaft als Ganzheit, die Sozialintegration dagegen auf die Integration der Akteure (bzw. der von ihnen gebildeten Gruppen) ,in“ das System hinein“(Esser 2001: S. 3) ${ }^{15}$.

Für Esser beschreibt die Sozialintegration den „Einbezug der Akteure in das gesellschaftliche Geschehen, etwa in Form der Gewährung von Rechten, des Erwerbs von Sprachkenntnissen, der Beteiligung am Bildungssystem und am Arbeitsmarkt, der Entstehung sozialer Akzeptanz, der Aufnahme von interethnischen Freundschaften, der Beteiligung am öffentlichen und am politischen Leben und auch der emotionalen Identifikation mit dem Aufnahmeland“ (Esser 2001: 8).

Wie bereits im vorangegangenen Unterkapitel zu Ergebnissen von Integration beschrieben, sind das Herkunfts- und das Aufnahmeland mögliche Bezugspunkte der Sozialintegration. Mit Hilfe dieser Bezugspunkte lassen sich vier Fälle von Integration ableiten. Diese sind die Mehrfachintegration (Integration in beide Kontexte), Marginalität (Integration in keinen Kontext), Assimilation (Aufnahmeland) und Segmentation (Herkunftsland) (Esser 2001: S. 73). Nach Esser stellt die Mehrfachintegration einen seltenen Sonderfall dar, weshalb nur die Assimilation ein politisches Ziel darstellen kann. Assimilation bedeutet in diesem Zusammenhang jedoch nicht, dass eine Anpassung stattfindet, sondern wird als Verringerung systematischer Unterschiede (in bspw. Einkommen, Bildung) verstanden (Esser 2001: S. 74).

Insgesamt sind vier Dimensionen von Sozialintegration zu unterscheiden, die kausale Beziehungen aufweisen und auch für eine gelingende Systemintegration von Bedeutung sein können. Die zu unterscheidenden Dimensionen bezeichnet Esser als Kulturation, Platzierung, Interaktion und Identifikation (Esser 2001: 8).

Unter Kulturation versteht Esser die Aneignung von Wissen und Kompetenzen, die für ein erfolgreiches Agieren und Interagieren in der Aufnahmegesellschaft nötig sind. Die Kulturation bezieht sich dabei insbesondere auf Fähigkeiten für die Bewältigung typischer Situationen. Von besonderer Bedeutung sind hierbei Fertigkeiten sprachlicher Art (Esser 2001: 8/9).

Die Platzierung beschreibt Esser als wahrscheinlich wichtigste Form der Integration in eine Gesellschaft. Diese umfasst „die Besetzung einer bestimmen gesellschaftlichen Position durch

\footnotetext{
${ }^{15}$ Im Folgenden wird nur auf die Sozialintegration näher eingegangen, da diese für die Untersuchung von Bedeutung ist.
} 
einen Akteur“ (Esser 2001: 9). Insgesamt ist unter Platzierung die Eingliederung in ein bestehendes soziales System zu verstehen. Im Rahmen dieser Eingliederung werden bestimmte Rechte verliehen (Staatsbürgerschaft und damit zusammenhängend das Wahlrecht, berufliche Positionen, Eröffnung sozialer Gelegenheiten). Esser vergleicht den Vorgang der Platzierung mit dem Marktgeschehen. Durch Fertigkeiten und Ressourcen machen die Akteurinnen und Akteure eine Art „Angebot“, wie beispielsweise die Arbeitskraft, welches von anderen Akteurinnen und Akteuren nachgefragt werden kann. Dabei ist der Nachfragende vor allem an seinen instrumentellen Vorteilen interessiert. Es können aber auch nicht-instrumentelle Gründe, wie beispielsweise Sympathie, eine Rolle spielen (Esser 2001: 9/10). Für Esser ist die Platzierung eng mit der Kulturation verbunden. So werden über die Platzierung bestimmte Kompetenzen erworben, die für eine erfolgreiche Kulturation von Bedeutung sind, aber auch über die Kulturation Kompetenzen erlernt, die für die Platzierung in der Gesellschaft eine Rolle spielen (Esser 2001: 10). Um die Bedeutung der Platzierung herauszustellen, schreibt Esser: „Die Plazierung auf den (möglichst auch: zentralen) Positionen einer Gesellschaft ist der Schlüssel für jede nachhaltige Sozialintegration. Und wegen der engen Verbindungen der Plazierung zum Vorgang der Kulturation, insbesondere über die Plazierung in die Bildungsinstitutionen und den Erwerb generell verwertbarer Fertigkeiten und Kompetenzen, gewinnt auch die Kulturation eine hohe, wenngleich etwas indirektere Bedeutung“ (Esser 2001: $10)$.

Nach Esser findet die Platzierung in den nicht-formellen Bereichen einer Gesellschaft über die Interaktion statt. Zum einen spielt dabei eine emotionale Komponente eine Rolle und zum anderen existieren „technische“ Bedingungen der Interaktion. In emotionaler Hinsicht wirken sich „Dissonanzen“ zwischen bestimmten Personen hemmend aus. Diese „Dissonanzen“ können nur überwunden werden, sofern gegenseitige Interessen ,,materieller“ Art an der Beziehung bestehen. Solche Barrieren, die auch kultureller Natur sein können, werden beispielsweise eher überwunden, wenn eine Person über bestimmte Ressourcen verfügt, die sie für andere interessant macht. „Technische“ Bedingungen für die Interaktion können zum einen Gelegenheiten des Zusammentreffens darstellen. So existieren bei beispielsweiser starker ethnischer Segregation kaum Möglichkeiten des Zusammentreffens und damit der interethnischen Interaktion. Zum anderen spielen auch die kulturellen Fähigkeiten der Akteurinnen und Akteure, und dabei vor allem die Beherrschung der Sprache, eine wichtige Rolle. Gleichzeitig hilft eine erfolgreiche Interaktion wiederrum beim Erlernen einer Sprache und damit der Kulturation. Esser spricht deshalb von einer sich selbst verstärkenden Beziehung von Kulturation, Interaktion und auch Platzierung, welche zur Segmentation bzw. Entwicklung 
eigenethnischer Gemeinden führen kann (Esser 2001: 11). Insgesamt kommt Esser zu dem Schluss, dass die interethnische Interaktion speziellen Bedingungen unterliegt und vor allem das Vorhandensein spezieller Kapitalien bei den Akteurinnen und Akteuren von Bedeutung ist (Esser 2001: 12).

Im Rahmen der Identifikation sieht die Person das soziale Gebilde schließlich als Einheit und identifiziert sich mit diesem. Dabei kommt es zu einer kollektiven Orientierung wie beispielsweise dem Gefühl von Nationalstolz oder einem Wir-Gefühl. Esser unterscheidet dabei drei Formen der Identifikation, die unterschiedliche Intensitäten abbilden und auch gleichzeitig vorhanden sein können. Diese sind die emphatische Wertintegration, der Bürgersinn und die Hinnahme des Systems (Esser 2001: 12). Die emphatische Wertintegration beschreibt die Identifikation mit dem sozialen System als „Kollektiv“, wobei es auch zur emotionalen Unterstützung zentraler Institutionen kommt. Es entsteht beispielsweise ein Gefühl der Solidarität und die Identifikation wird emotional erlebt (Esser 2001: 12/13).

Dass eine so starke Identifikation in modernen Gesellschaften bestehen muss, ist umstritten. Esser führt daher auch die Konzepte des Bürgersinns und der Hinnahme ein, um schwächere Formen der Identifikation darzustellen. Im Rahmen des Bürgersinns ,geht es dabei nur in einem sehr abstrakten Sinn um die Unterstützung einer kollektiven Gesellschaft“ (Esser 2001: 13). Vielmehr geht es um den Schutz individueller Freiheiten und der Freiheit von „kollektiven“ Zwängen, für deren Erhalt die Bürgerinnen und Bürger die spezielle Form der Verfassung unterstützen. Insgesamt geht es somit auch um „die Abwehr“ gesinnungsethischer und fundamentalistisch-kollektivistischer Ansprüche (Esser 2001: 13).

Unter Hinnahme versteht Esser Orientierungen, die lediglich auf Interessen-Konstellationen und Möglichkeiten der Akteurinnen und Akteure basieren. Hierbei unterscheidet Esser wiederrum zwischen Verkettungs- und Deferenzintegration. Die Verkettungsintegration beruht auf Überkreuzungen und Inkonsistenzen von Orientierungen. Aufgrund dieser Überkreuzungen und Inkonsistenzen ist es den Akteurinnen und Akteuren nicht möglich, sich zu größeren, systemdesintegrierenden Gruppen zusammenzuschließen. Gleichzeitig wird das System auch hingenommen, da es Vorteile für die Akteurinnen und Akteure (beispielsweise Wohnraum etc.) bietet. Im Gegensatz dazu beschreibt die Deferenzintegration eine Hinnahme des Systems aufgrund von Aussichtslosigkeit (Esser 2001: 14). 


\subsubsection{Friedrich Heckmann}

Heckmann definiert den Prozess der Integration wie folgt: "Integration ist der Mitgliedschaftserwerb von Zuwanderern in den Institutionen, sozialen Beziehungen und sozialen Milieus der Aufnahmegesellschaft. Integration als Prozess der Mitgliedschaftswerdung und Angleichung der Lebensverhältnisse entwickelt sich schrittweise entlang der Dimensionen der strukturellen, kulturellen, sozialen und identifikativen Integration. Sie erfordert Integrationsleistungen der Migranten und bedarf der Offenheit und Förderung seitens der Aufnahmegesellschaft. Sie ist somit ein wechselseitiger, wenngleich nicht gleichgewichtiger Prozess, der über Generationen verläuft. Integration als Zustand und Ergebnis soll heißen, dass volle und gleichberechtigte gesellschaftliche Mitgliedschaft einer zugewanderten Gruppe in der Aufnahmegesellschaft besteht und sich die Lebensverhältnisse angeglichen haben. Ethnische Herkunft und Migrationshintergrund spielen für Ressourcenverteilung und die Strukturierung sozialer Beziehungen keine Rolle mehr“ (Heckmann 2015: 82).

Im Rahmen von Dimensionen unterscheidet Heckmann somit insgesamt vier Ebenen von Integration. Diese umfassen die strukturelle, kulturelle, soziale und identifikative Integration und sind an den Dimensionen von Hartmut Esser (2001) orientiert. Jedoch differenzieren Heckmann et al. weiter aus, was unter den einzelnen Dimensionen verstanden werden kann (Heckmann et al. 2000: 8). Außerdem formuliert der Integrationsprozess dabei nicht nur Anforderungen an Migrantinnen und Migranten, sondern auch an die Aufnahmegesellschaft (Heckmann et al. 2000: 12).

Die strukturelle Integration umfasst nach Heckmann et al. den Status in den Kerninstitutionen Wirtschaft/Arbeitsmarkt, Bildungs- und Qualifikationssysteme, Wohnungsmarkt sowie in der politischen Gemeinschaft (Heckmann et al. 2000: 8). Zentrale Stellung hat dabei der Zugang zum Arbeitsmarkt, da dieser für den gesamten Integrationsprozess von Bedeutung ist. „Wirtschaftliche Tätigkeit verschafft Einkommen, aber auch soziale Beziehungen, Sozialprestige und Selbstwertgefühl (Heckmann et al. 2000: 12). Dabei ist nicht nur der gleiche Zugang zum Arbeitsmarkt von Bedeutung, sondern auch gleiche Arbeitsbedingungen (Heckmann 1997: 2). Der chancengleiche Zugang zum Wohnungsmarkt spielt vor allem in Bezug auf interethnische Begegnungschancen eine Rolle. Im Falle von starker Wohnsegregation sind die Chancen auf interethnischen Kontakt eher gering (Heckmann et al. 2000: 13/14). Ein weiterer wichtiger Faktor ist der Zugang zur Staatsangehörigkeit und damit die rechtliche Gleichstellung. Ein Teil der rechtlichen Gleichstellung kann auch schon vor dem 
Zugang der Staatsangehörigkeit durch eine arbeits- und sozialrechtliche Gleichstellung gewährleistet werden (Heckmann et al. 2000: 14).

Die kulturelle Integration umfasst das Erlernen kognitiver Fähigkeiten sowie die Kenntnis der Kultur der Aufnahmegesellschaft bzw. des Einwanderungslandes. Werte, Normen und Einstellungen werden internalisiert und darüber hinaus kommt es auch zu einer Veränderung des Glaubenssystems. Zu betonen ist hierbei, dass es dabei nicht nur zu Veränderungen auf Seiten der Migrantinnen und Migranten kommt, sondern auch Veränderungen in der aufnehmenden Gesellschaft stattfinden, indem ethnische Grenzen aufgeweicht werden (Heckmann 1997: 4). Die kulturelle Integration, die Heckmann et al. auch als Akkulturation bezeichnen, findet dabei in öffentlich-staatlichen Institutionen (Arbeit, Erziehungs- und Bildungssystem, Medien) statt, während ethnische Praktiken im privaten Raum verbleiben (Heckmann et al. 2000: 14). Für ihre Untersuchung führen Heckmann et al. einige Indikatoren für die kulturelle Integration ein. Einer der wichtigsten Indikatoren ist dabei die Sprachkompetenz in der Sprache der Aufnahmegesellschaft sowie des Herkunftslandes bzw. des Herkunftslandes der Eltern, da durch die Verwendung der Herkunftssprache bzw. der Sprache der Eltern die Einstellung zur Herkunftskultur dargestellt werden kann (Heckmann et al. 2000: 25). Ein weiterer Indikator ist die Mediennutzung. Durch die Medien werden Einstellungen und Werte vermittelt, wodurch sie eine wichtige Rolle bei deren Internalisierung spielen. Daher ist es von Bedeutung, ob Migrantinnen und Migranten auf Fernsehsender, Radiosender oder Printmedien der Herkunftskultur zurückgreifen oder das Angebot der Aufnahmegesellschaft nutzen (Heckmann et al. 2000: 26-31). Darüber hinaus führen Heckmann et al. das Interesse an Politik (Heckmann et al. 2000: 33) sowie die Ess- und Trinkgewohnheiten als Indikatoren ein. Dabei spielen beispielsweise bei Musliminnen und Muslimen der Verzicht auf Alkohol und Schweinefleisch eine Rolle ((Heckmann et al. 2000: 35-37). Ein weiterer Indikator ist die Einstellung zu Familie und Partnerschaft. Im Rahmen dieses Indikators ist vor allem die Einstellung zur Verteilung von Geschlechterrollen wichtig. Aber auch die Einstellung zu Scheidung, Größe der Familie, Kinderzahl und dem Leben ohne Trauschein spielen eine Rolle (Heckmann et al. 2000: 37-41). Der letzte eingeführte Indikator ist die Bedeutung der Religion. Hier stellen Heckmann et al. auf Religionszugehörigkeit, Religiosität, die Wichtigkeit religiöser Feste, Bedingungen von Religion für die Wahl der Partnerinnen und Partner sowie auf die Mitgliedschaft in religiösen Organisationen ab (Heckmann et al. 2000: 41-46). 
Die nächste von Heckmann beschriebene Dimension ist die soziale Integration. Soziale Integration umfasst die sozialen Kontakte und Gruppenmitgliedschaften einer Person und die Frage, ob diese primär innerhalb der eigenen ethnischen Gruppe stattfinden oder darüber hinausgehen (Heckmann 1997: 6). So stellt sich beispielsweise bei Vereinen die Frage, ob diese am Herkunftsland orientiert und damit eigenethnische Vereine sind, wodurch wenig Interaktion über die eigene Primärgruppe hinaus stattfinden kann. Die soziale Integration ist stark an die „Offenheit und Bereitschaft, solche Mitgliedschaften $\mathrm{zu}$ akzeptieren und Vorurteile und Diskriminierung abzubauen“, gebunden (Heckmann et al. 2000: 15). Indikatoren, die unter diese Dimension gefasst werden, sind individuelle Freundschaften, Partnerschaften, Wahl der Ehepartnerin bzw. des Ehepartners sowie Vereinsmitgliedschaften bzw. die Partizipation in Organisationen (Heckmann et al. 2000: 47).

Die letzte Dimension stellt die identifikative Integration dar und beschreibt subjektive Gefühle, wie ein Zugehörigkeitsgefühl zu einer ethnischen oder nationalen Gemeinschaft. Heckmann stellt jedoch auch fest, dass diese komplexe Form der Integration nur schwer durch standardisierte Befragungen dargestellt werden kann (Heckmann 1997: 6). Darüber zeigt sich, dass diese Dimension der Integration langsamer abläuft als die zuvor dargestellten Dimensionen und bestimmte Voraussetzungen vorliegen müssen. Zu diesen Voraussetzungen gehört auch ein Identifikationsangebot durch die Aufnahmegesellschaft (Heckmann et al. 2000: 16). Indikatoren für die identifikative Integration sind Zugehörigkeits- und Heimatgefühle, wobei vor allem die primäre Selbstidentifikation eine Rolle spielt, sowie Besuche/Urlaub im Herkunftsland und Rückkehrabsichten (Heckmann et al. 2000: 57-65).

\subsubsection{Bielefelder Desintegrationsansatz nach Anhut und Heitmeyer}

Der Bielefelder Desintegrationsansatz dient der Erklärung von Phänomenen wie Gewalt(kriminalität), Rechtsextremismus, ethnisch-kulturellen Konflikten sowie der Abwertung und Abwehr schwacher Gruppen. Diese Phänomene stellen dabei Verarbeitungsmuster von Zuständen der Desintegration dar (Anhut/Heitmeyer 2009: 212). Zentral innerhalb des Ansatzes ist das Erreichen von Anerkennung, welche durch soziale Integration erlangt werden kann (Anhut/Heitmeyer 2009: 220). Unter sozialer bzw. gesellschaftlicher Integration wird innerhalb des Ansatzes „ein gelungenes Verhältnis von Freiheit und Bindung“ verstanden (Anhut/Heitmeyer 2009: 220). Dabei werden drei Integrationsdimensionen unterschieden. Diese sind die individuell-funktionale Systemintegration, die kommunikativ-interaktive Sozialintegration und die kulturell-expressive Sozialintegration (Anhut/Heitmeyer 2009: 221). 
Die individuell-funktionale Systemintegration umfasst die Teilhabe an materiellen sowie kulturellen Gütern und führt zu einer Anerkennung der Position. Dabei ist der Zugang zu Arbeits-, Wohnungs- und Konsummarkt bedeutend, aber auch die Zufriedenheit mit der eigenen beruflichen und sozialen Situation (Anhut/Heitmeyer 2009: 220). Probleme ergeben sich innerhalb dieser Dimension bei vorhandenen sozialen Spaltungen, die dazu führen, dass Zugangschancen ungleich verteilt sind. Dies führt gepaart mit dem Druck erfolgreich zu sein, Wettbewerbs- oder Konkurrenzdenken zu Resignation, Ohnmacht und/oder Wut (Anhut/Heitmeyer 2009: 222).

Eine weitere Integrationsdimension im Bielefelder Desintegrationsansatz ist die kommunikativ-interaktive Sozialintegration. Innerhalb dieser Dimension geht es um den Ausgleich im Konflikt stehender Interessen und die damit einhergehende moralische Anerkennung. Der Ausgleich im Konflikt stehender Interessen umfasst „die Einhaltung basaler, die moralische Gleichwertigkeit des (politischen) Gegners gewährleistende, demokratische Prinzipien, die von den Beteiligten als fair und gerecht bewertet werden“" (Anhut/Heitmeyer 2009: 220). Anhut und Heitmeyer beschreiben innerhalb dieser Dimension eine objektive und eine subjektive Subdimension, die durch die vorhandenen demokratischen Prinzipien und deren Ausgestaltung sowie Aushandlung bedingt werden. Die objektive Subdimension bezieht sich auf Teilnahmechancen, während die subjektive Subdimension für die eigene Teilnahmebereitschaft steht. Beide Subdimensionen werden durch den Ausgleich konfligierender Interessen, in Form der Einhaltung demokratischer Prinzipien, bedingt. Weiter geht es innerhalb dieser Dimension auch um die Anerkennung von Grundnormen wie beispielsweise Fairness und Solidarität (Anhut/Heitmeyer 2009: 221).

Als letzte Dimension beschreiben die Autoren die kulturell-expressive Sozialintegration. Hierbei handelt es sich um emotionale und expressive Beziehungen, die einerseits der Sinnstiftung und andererseits der Selbstverwirklichung dienen, um emotionale Anerkennung $\mathrm{zu}$ erreichen. Auch die Sicherung sozio-emotionalen Rückhalts spielt innerhalb dieser Dimension eine Rolle, da dadurch Krisen vermieden werden können. Darüber hinaus geht es in dieser Dimension um die Anerkennung durch das Kollektiv und die soziale Umwelt sowie die Akzeptanz kollektiver Identitäten und deren Symbole durch andere Akteurinnen und Akteure (Anhut/Heitmeyer 2009: 220/221).

Werden die drei vorgestellten Dimensionen erfüllt, kommt es zu positioneller, moralischer sowie emotionaler Anerkennung und das Individuum definiert sich als einem Kollektiv zugehörig (Anhut/Heitmeyer 2009: 222). 


\subsubsection{Gegenüberstellung der Dimensionen von Integration der theoretischen Konzepte}

Ein Vergleich der einzelnen Ansätze zeigt ${ }^{16}$, dass überwiegend vergleichbare Dimensionen von Integration angesprochen werden. Dabei wird in den meisten Fällen auf insgesamt vier Dimensionen abgestellt (Vgl. Eisenstadt 1954, Esser 2001, Heckmann et al. 2000). Diese umfassen eine kulturelle, soziale, identifikative sowie eine strukturelle Komponente. Innerhalb der Ansätze sind die einzelnen Dimensionen der Integration jedoch unterschiedlich weit differenziert. Am weitesten ins Detail geht dabei der Ansatz von Heckmann et al., der die einzelnen Dimensionen ausführlich mit unterschiedlichen Indikatoren beschreibt (Vgl. Heckmann et al. 2000).

Neben den vergleichbaren Dimensionen wird auch deutlich, dass manche Integrationsaspekte in mehreren Konzepten eine Rolle spielen, während andere nur die Beachtung einzelner Autorinnen und Autoren finden. So werden im Rahmen der Kulturation überwiegend Aspekte beschrieben, die die Aneignung von Wissen und Kompetenzen für die Bewältigung typischer Situationen beinhalten. Oft wird dabei Bezug auf die Sprache und Werte, Normen und Einstellungen genommen. Darüber hinaus findet sich bei Heckmann et al. (2000) jedoch auch ein weit differenziertes Set an Aspekten, die zusätzlich zu den anderen Ausarbeitungen noch die Mediennutzung, das Interesse an Politik, Ess- und Trinkgewohnheiten, die Einstellung zu Familie und Partnerschaft und die Bedeutung von Religion beinhalten. Anhut und Heitmeyer (2009) beschreiben in ihrer Dimension der kommunikativ-interaktiven Sozialintegration ebenfalls die Anerkennung von Grundnormen. Darüber hinaus bringen sie als einzige Autoren auch noch einen Aspekt ein, der sich auf das Zusammenleben in der Gesellschaft bezieht. Dieser umfasst den Ausgleich konfligierender Interessen, die Teilnahmechancen sowie die Teilnahmebereitschaft in einer Gesellschaft bedingen. Dadurch scheinen bei Anhut und Heitmeyer, die insgesamt auch nur drei Dimensionen behandeln, innerhalb der Dimension der kommunikativ-interaktiven Sozialintegration Aspekte der Kulturation und Interaktion kombiniert zu sein. Davon abzugrenzen ist die Teilhabe an materiellen und kulturellen Gütern, die nach Anhut und Heitmeyer einer personalen Ebene und der Dimension der individuellfunktionalen-Systemintegration zuzuordnen ist.

Innerhalb der Dimension der Interaktion liegt der Fokus auf interethnischen Kontakten. Esser (2001) beschreibt außerdem die Gelegenheiten des Zusammentreffens als wichtigen Aspekt. Dieser Aspekt findet sich auch bei Heckmann et al. (2000) in Form der Partizipation in

\footnotetext{
${ }^{16}$ Siehe nachfolgende Tabelle 5
} 
Organisationen. Darüber hinaus führen Heckmann et al. noch das politische Interesse an. Auch im Rahmen dieser Dimension können Anhut und Heitmeyer (2009) weiterführende Aspekte anbringen. Diese sind die Anerkennung durch das Kollektiv und die soziale Umwelt, die Akzeptanz kollektiver Identitäten und derer Symbole durch andere sowie die Sicherung sozioemotionalen Rückhalts.

Die Dimension der Identifikation besteht bei Anhut und Heitmeyer (2009) nicht, da sich ihr Ansatz nicht explizit auf Migrantinnen und Migranten bezieht und diese Dimension daher keine Rolle spielt. In allen anderen Ansätzen spielt die Identifikation in Form einer kollektiven Orientierung oder von Zugehörigkeitsgefühlen eine Rolle. Heckmann et al. (2000) beschreiben außerdem noch den Bezug zum Heimatland und die Rückkehrabsicht als Aspekte.

Die meisten Überschneidungen zwischen den unterschiedlichen Ausführungen finden sich bei der Dimension der Platzierung. Hier werden von den meisten Autorinnen und Autoren die berufliche Stellung bzw. der Zugang zum Arbeitsmarkt, die rechtliche Stellung und Bildung angesprochen. Auch das „Wohnen“ bzw. der Zugang zum Wohnungsmarkt findet sich in einigen der vorgestellten Ausarbeitungen, wohingegen das Einkommen nur bei HoffmannNowotny (1973) als relevanter Aspekt behandelt wird. Esser (2001) führt außerdem die Eröffnung sozialer Gelegenheiten und die Besetzung einer bestimmten Position in der Gesellschaft an. Die Position spielt auch bei Heckmann et al. (2000), in Form der Stellung in der politischen Gemeinschaft, eine Rolle. Anhut und Heitmeyer (2009) formulieren auch hier noch darüberhinausgehende Aspekte. Diese umfassen den Zugang zum Konsummarkt, die Teilhabe an materiellen und kulturellen Gütern sowie die Zufriedenheit mit der beruflichen und sozialen Stellung.

Die erläuterten Gemeinsamkeiten und Unterschiede der Konzepte sind in der nachfolgenden Tabelle 5 nochmals anhand einer Gegenüberstellung aufgeführt. Im weiteren Verlauf der Arbeit werden, wie in der Tabelle dargestellt, die Dimensionsbezeichnungen von Esser verwendet. 
Tabelle 5: Gegenüberstellung der Aspekte und Dimensionen von Integration anhand der ausgewählten theoretischen Konzeptionen

\begin{tabular}{|c|c|c|c|c|c|}
\hline Eisenstadt & Hoffmann-Nowotny & Esser & Heckmann & Anhut/Heitmeyer & Bezeichnung \\
\hline $\begin{array}{l}\text { Adaptive Integration } \\
\text { - } \text { Aufbau sozialer } \\
\text { Kontakte } \\
\text { - Positive Identifikation } \\
\text { mit Werten und } \\
\text { Strukturen }\end{array}$ & - Soziale Interaktion & $\begin{array}{l}\text { Interaktion } \\
\text { - Aufbau interethnischer } \\
\text { Kontakte } \\
\text { - Gelegenheiten des } \\
\quad \text { Zusammentreffens }\end{array}$ & $\begin{array}{l}\text { Soziale Integration } \\
\text { - Interethnische Kontakte } \\
\text { (Freundschaften, } \\
\text { Partnerschaften, Wahl } \\
\text { Ehepartner) } \\
\text { - } \quad \text { Partizipation in } \\
\text { Organisationen } \\
\text { - } \quad \text { Politisches Interesse }\end{array}$ & $\begin{array}{l}\text { Kommunikativ-interaktive } \\
\text { Sozialintegration } \\
\text { - } \quad \text { Ausgleich } \\
\text { konfligierender } \\
\text { Interessen } \\
\text { - } \quad \text { Teilnahmechancen } \\
\text { - } \quad \text { Teilnahmebereitschaft } \\
\text { Kulturell-expressive } \\
\text { Sozialintegration } \\
\text { - } \quad \text { Emotionale und } \\
\text { expressive Beziehungen } \\
\text { - Sicherung sozio- } \\
\text { emotionalen Rückhalts } \\
\text { - Anerkennung durch } \\
\text { Kollektiv und soziale } \\
\text { Umwelt } \\
\text { - Akzeptanz kollektiver } \\
\text { Identitäten und deren } \\
\text { Symbole durch Andere }\end{array}$ & Interaktion \\
\hline
\end{tabular}




\begin{tabular}{|c|c|c|c|c|c|}
\hline Eisenstadt & Hoffmann-Nowotny & Esser & Heckmann & Anhut/Heitmeyer & \\
\hline $\begin{array}{l}\text { Solidarische Integration } \\
\text { - } \quad \text { Identifikation und } \\
\text { Solidarisierung mit } \\
\text { Wertvorstellungen } \\
\text { - } \quad \text { Zugehörigkeitsgefühl } \\
\text { - } \quad \text { Aktive Partizipation }\end{array}$ & & $\begin{array}{l}\text { Identifikation } \\
\text { - } \quad \text { Kollektive Orientierung } \\
\text { - } \quad \text { Bürgersinn } \\
\text { - } \quad \text { Hinnahme }\end{array}$ & $\begin{array}{l}\text { Identifikative Integration } \\
\text { - } \quad \text { Zugehörigkeits- und } \\
\text { Heimatgefühle } \\
\text { - } \quad \text { Rückkehrabsicht } \\
\text { - } \quad \text { Bezug zum „Heimatland“ }\end{array}$ & & Identifikation \\
\hline $\begin{array}{l}\text { Instrumentale } \\
\text { Integration } \\
\text { - Übernahme von } \\
\text { Rollen im } \\
\text { Wirtschaftsleben }\end{array}$ & $\begin{array}{l}\text { Partizipation an der } \\
\text { Struktur der } \\
\text { Gesellschaft } \\
\text { - } \quad \text { Rechtliche Stellung } \\
\text { - } \quad \text { Bildung } \\
\text { - } \quad \text { Berufliche Stellung } \\
\text { - } \quad \text { Einkommen } \\
\text { - } \quad \text { Wohnen }\end{array}$ & $\begin{array}{ll}\text { Platzierung } \\
\text { - } & \text { Rechtliche Stellung } \\
\text { - } & \text { Bildung } \\
\text { - } & \text { Berufliche Position } \\
\text { - } & \text { Eröffnung sozialer } \\
& \text { Gelegenheiten } \\
\text { - } & \text { Besetzung einer } \\
& \text { bestimmten Position }\end{array}$ & $\begin{array}{l}\text { Strukturelle Integration } \\
\text { - } \quad \text { Rechtliche Stellung, arbeits- } \\
\text { und sozialrechtliche } \\
\text { Gleichstellung } \\
\text { - } \quad \text { Bildungs- und } \\
\text { Qualifikationssystem } \\
\text { - } \quad \text { Arbeitsmarkt } \\
\text { - } \quad \text { Wohnungsmarkt } \\
\text { - } \quad \text { Stellung politische } \\
\text { Gemeinschaft }\end{array}$ & $\begin{array}{l}\text { Individuell-funktionale- } \\
\text { Systemintegration } \\
\text { - } \quad \text { Teilhabe an materiellen } \\
\text { und kulturellen Gütern } \\
\text { - Zugang Arbeitsmarkt } \\
\text { - } \quad \text { Zugang } \\
\text { Wohnungsmarkt } \\
\text { - Zugang Konsummarkt } \\
\text { - Zufriedenheit mit } \\
\quad \text { beruflicher und sozialer } \\
\quad \text { Stellung } \\
\end{array}$ & Platzierung \\
\hline
\end{tabular}


Betrachtet man die fünf vorgestellten Konzepte in ihrer chronologischen Abfolge, fällt auf, dass die Faktoren von Eisenstadt (1951; 1952a; 1952b) durchgehend auf Leistung der Migrantinnen und Migranten aufbauen, obwohl auch ausgeführt wird, dass die Anpassung auch von der Bereitschaft der Aufnahmegesellschaft abhängt. So sollen für eine erfolgreiche Integration die Basisrollen in den Hauptinstitutionen erlernt werden, eine Identifikation und Solidarisierung mit Strukturen und Werten stattfinden, emotionale Ausdrucksformen verinnerlicht, soziale Kontakte aufgebaut und Rollen im Wirtschaftsleben übernommen werden. Dies rührt von der theoretischen Prägung der Migrationstheorie nach Eisenstadt, die sehr durch den Gedanken der Assimilation, in Form von Anpassung, beeinflusst ist (Vgl. Eisenstadt 1954). Im Gegensatz dazu stellt Hoffmann-Nowotny stark auf vorhandene Opportunitäten und die Verteilung/den Platz der Migrantinnen und Migranten innerhalb unterschiedlicher Subsysteme in der Aufnahmegesellschaft ab. So spielen hier lediglich Faktoren, die die Partizipation an der Struktur der Gesellschaft betreffen, eine Rolle (rechtliche Stellung, Bildung, berufliche Stellung, Einkommen, Wohnen, soziale Interaktion) (Vgl. Hoffmann-Nowotny 1973). In Essers Ansatz lassen sich schließlich sowohl Faktoren finden, die auf der Leistung von Migrantinnen und Migranten aufbauen (Aneignung von Wissen und Kompetenzen für die Bewältigung typischer Situationen, interethnische Kontakte, kollektive Orientierung) als auch vorhandene Opportunitäten in der Aufnahmegesellschaft (rechtliche Stellung, Bildung, berufliche Position, Eröffnung sozialer Gelegenheiten, Besetzung bestimmter Positionen) (Vgl. Esser 2001). Dies ist auch in der Arbeit von Heckmann et al. (2000) der Fall. Hier werden die einzelnen Dimensionen von Integration jedoch ausführlicher mit Indikatoren versehen.

Im Bielefelder Desintegrationsansatz nach Anhut und Heitmeyer sind schließlich nicht nur Leistungen auf Seiten der Migrantinnen und Migranten sowie vorhandene Opportunitäten zu finden, sondern auch Leistungen der Gesellschaft. Dabei zeigt sich innerhalb der einzelnen Adressatenebenen auch eine Bedeutung emotionaler Aspekte. So beziehen sich die Anerkennung von Grundnormen, der Ausgleich konfligierender Interessen und die Teilnahmebereitschaft auf Leistungen der Migrantinnen und Migranten. Die Aspekte Teilnahmechancen, Zugang zu Arbeitsmarkt, Wohnungsmarkt sowie Konsummarkt beziehen sich hingegen auf vorhandene Opportunitäten. Darüber hinaus, kommen als Leistungen der aufnehmenden Gesellschaft die Anerkennung sowie die Akzeptanz kollektiver Identitäten und deren Symbole hinzu. Emotionale Aspekte in diesem Ansatz sind das Vorhandensein emotionaler und expressiver Beziehungen, die Sicherung sozio-emotionalen Rückhalts sowie die Zufriedenheit mit der eigenen beruflichen und sozialen Stellung (Vgl. Anhut und Heitmeyer 2009). Neben einer Einordnung in verschiedene Dimensionen ist es somit auch möglich die 
Aspekte von Integration nach den Adressatinnen und Adressaten zu ordnen, an die sich die einzelnen Aspekte richten (vgl. Tabelle 6).

In der chronologischen Abfolge der Modelle ist so auch erkennbar, dass die vorgestellten Ansätze zwar vergleichbare Dimensionen von Integration ansprechen, jedoch wird innerhalb der Ansätze von der Vorstellung, Integration wäre nur auf Leistung der Migrantinnen und Migranten begründet, mit der Zeit Abstand genommen. Dies hängt wohl auch mit dem Diskurs über die Erwartungen an Integration, in Form von deren Ergebnis, zusammen. Hier herrschte, wie bereits dargestellt, lange Zeit eine Dominanz des Assimilationsgedankens. Neben der Entwicklung pluralistischer Konzepte kam es dabei im zeitlichen Verlauf auch zu einem veränderten Verständnis von Assimilation mit einer offeneren Ausgestaltung.

Anhut und Heitmeyer (2009), deren Ansatz nicht explizit auf Migrantinnen und Migranten bezogen ist, sondern Erklärung von Phänomenen wie Gewalt(-kriminalität), Rechtsextremismus, ethnisch-kulturellen Konflikten sowie der Abwertung und Abwehr schwacher Gruppen dient, können die vorgestellten Ansätze zusätzlich um Leistungen der aufnehmenden Gesellschaft sowie um emotionale Aspekte auf unterschiedlichen Adressatenebenen ergänzen.

Die unterschiedlichen Adressatenebenen, die in den Konzepten enthalten sind, werden in der nachfolgenden Tabelle 6 nochmals zusammenfassend dargestellt. Dabei ist zu beachten, dass sich manche Aspekte nicht trennscharf einer Adressatenebene zuordnen lassen. Diese Aspekte umfassen beispielsweise interethnische Kontakte, Partizipation in Organisationen, Bildungsstand und berufliche Stellung, da diese Aspekte nicht nur vom Verhalten der Migrantinnen und Migranten, sondern auch von Opportunitäten sowie dem Verhalten der Aufnahmegesellschaft abhängig sein können. Somit handelt es sich um eine erste Zuordnung der Aspekte zu Adressatenebenen. 
Tabelle 6: Darstellung der Adressatenebenen der ausgewählten theoretischen Konzeptionen

Eisenstadt

Hoffmann-Nowotny

Esser

Heckmann

Anhut/Heitmeyer

Anforderung an Migrantinnen und Migranten

- Übernahme

emotionaler

Ausdrucksformen

und Symbole im

Alltag

- Erlernen Basisrollen in Hauptinstitutionen

- Aufbau sozialer

Kontakte

- Positive

Identifikation mit

Werten und

Strukturen

- Identifikation und Solidarisierung mit Wertvorstellungen

- Zugehörigkeitsgefühl

- Aktive Partizipation

- Übernahme von

Rollen im

Wirtschaftsleben

\begin{tabular}{|c|c|c|}
\hline - $\quad$ Soziale Interaktion & $\begin{array}{l}\text { Aneignung von } \\
\text { Wissen und } \\
\text { Kompetenzen für } \\
\text { die Bewältigung } \\
\text { typischer } \\
\text { Situationen } \\
\text { (bspw. Sprache) } \\
\text { Kollektive } \\
\text { - Orientierung } \\
\text { - Bürgersinn } \\
\text { Hinnahme }\end{array}$ & $\begin{array}{ll}\text { - } & \text { Internalisierung von } \\
\text { Werten, Normen, } \\
\text { - } & \text { Sinstellungen } \\
\text { - } & \text { Mediennutzung } \\
\text { - } & \text { Interesse an Politik } \\
\text { - } & \text { Ess- und } \\
\text { - } & \text { Trinkgewohnheiten } \\
\text { Einstellung zu } \\
\text { Familie und } \\
\text { Partnerschaft } \\
\text { Bedeutung von } \\
\text { - Religion } \\
\text { Partizipation in } \\
\text { Organisationen } \\
\text { - Politisches Interesse } \\
\text { Zugehörigkeits- und } \\
\text { Heimatgefühle } \\
\text { - Rückkehrabsicht } \\
\text { Bezug zum } \\
\text { „Heimatland“ }\end{array}$ \\
\hline
\end{tabular}

- Anerkennung Grundnormen

- Teilnahmebereitschaft

- Zufriedenheit mit beruflicher und sozialer Stellung

\section{Opportunitäten in der Aufnahmegesellschaft}

\begin{tabular}{|c|c|c|c|c|}
\hline \multicolumn{5}{|c|}{ Opportunitäten in der Aufnahmegesellschaft } \\
\hline & $\begin{array}{ll}\text { - } & \text { Rechtliche Stellung } \\
\text { - } & \text { Bildung } \\
\text { - } & \text { Berufliche Stellung } \\
\text { - } & \text { Einkommen } \\
\text { - } & \text { Wohnen }\end{array}$ & $\begin{array}{ll}\text { - } & \text { Aufbau } \\
\text { interethnischer } \\
\text { Kontakte } \\
\text { - } & \text { Gelegenheiten } \\
\text { des Zusammen- } \\
\text { treffens } \\
\text { - } \quad \text { Rechtliche } \\
\text { Stellung } \\
\text { - } \quad \text { Bildung } \\
\text { - } \text { Berufliche } \\
\text { - } \text { Position } \\
\text { Eröffnung } \\
\text { sozialer } \\
\text { Gelegenheiten } \\
\text { Besetzung einer } \\
\text { bestimmten } \\
\text { Position }\end{array}$ & $\begin{array}{l}\text { Interethnische } \\
\text { Kontakte } \\
\text { (Freundschaften, } \\
\text { Partnerschaften, } \\
\text { Wahl Ehepartner) } \\
\text { - auch die } \\
\text { aufnehmende } \\
\text { Gesellschaft } \\
\text { verändert sich } \\
\text { Rechtliche Stellung, } \\
\text { arbeits- und } \\
\text { sozialrechtliche } \\
\text { Gleichstellung } \\
\text { Bildungs- und } \\
\text { Qualifikations- } \\
\text { system } \\
\text { Arbeitsmarkt } \\
\text { Wohnungsmarkt } \\
\text { - Stellung politische } \\
\text { Gemeinschaft }\end{array}$ & $\begin{array}{ll}\text { - } & \text { Ausgleich } \\
\text { konfligierender } \\
\text { Interessen } \\
\text { - } \quad \text { Teilnahme- } \\
\text { chancen } \\
\text { - Teilhabe an } \\
\text { materiellen und } \\
\text { kulturellen } \\
\text { Gütern } \\
\text { - Zugang } \\
\text { Arbeitsmarkt } \\
\text { Zugang } \\
\text { Wohnungsmarkt } \\
\text { Zugang } \\
\text { Konsummarkt } \\
\text { Emotionale und } \\
\text { expressive } \\
\text { Beziehungen } \\
\text { Sicherung sozio- } \\
\text { emotionalen } \\
\text { Rückhalts }\end{array}$ \\
\hline \multicolumn{5}{|c|}{ Leistungen der Aufnahmegesellschaft } \\
\hline & & & & $\begin{array}{ll}\text { - Anerkennung } \\
\text { durch Kollektiv } \\
\text { und soziale } \\
\text { Umwelt } \\
\text { - } \\
\text { Akzeptanz } \\
\text { kollektiver } \\
\text { Identitäten und } \\
\text { deren Symbole } \\
\text { durch Andere }\end{array}$ \\
\hline
\end{tabular}

Quelle: Eigene Darstellung anhand der ausgewählten theoretischen Konzeptionen. 
Eine weitere Betrachtung der in den Ansätzen angesprochenen Aspekte zeigt die Möglichkeit auf, Aspekte, die im Grunde auf das gleiche Konstrukt abzielen, unter Oberbegriffen zusammenzufassen. Insgesamt konnten die folgenden acht Oberbegriffe identifiziert werden, die als zu bewertende Aspekte in die spätere Befragung eingehen sollten:

- Aneignung von Wissen und Werten

- Kontakte

- Zugehörigkeit/Identifikation

- Partizipation

- Akzeptanz/Anerkennung

- Zufriedenheit

- Transformation

- Stellung in der Gesellschaft

Es zeigt sich, dass die eigentlichen Oberbegriffe unterschiedlich weit differenziert werden können. So zeigt die nachfolgende Tabelle 7, dass unter die Oberkategorie „Aneignung von Wissen und Werten“ unterschiedlich viele Aspekte summiert werden können und die Autorinnen und Autoren somit unterschiedlich weit ins Detail gehen. Während Eisenstadt (1953) relativ unspezifisch bleibt, sind die Aspekte bei Heckmann et al. (2000) stark differenziert. Es stellt sich somit die Frage, wie die Oberkategorie „Aneignung von Wissen und Werten" und auch die anderen Oberkategorien operationalisiert werden sollen, da sie in ihrer Ausgestaltung als Oberbegriffe zu unspezifisch und abstrakt für die quantitative Befragung sind (Siehe hierzu Kapitel 5.2.1 Operationalisierung im Fragebogen). 
Tabelle 7: Zusammenfassung der Aspekte zum Oberbegriff Aneignung von Wissen und Werten

\begin{tabular}{|c|c|c|c|c|}
\hline Eisenstadt & $\begin{array}{l}\text { Hoffmann- } \\
\text { Nowotny }\end{array}$ & Esser & Heckmann & Anhut/Heitmeyer \\
\hline 1. Aneignung von & issen und W & rten & & \\
\hline $\begin{array}{l}\text { Übernahme } \\
\text { emotionaler } \\
\text { Ausdrucks- } \\
\text { formen und } \\
\text { Symbole im } \\
\text { Alltag } \\
\text { Erlernen von } \\
\text { Basisrollen in } \\
\text { Haupt- } \\
\text { institutionen }\end{array}$ & 1 & $\begin{array}{l}\text { - Aneignung } \\
\text { von Wissen } \\
\text { und Kompe- } \\
\text { tenzen für } \\
\text { die } \\
\text { Bewältigung } \\
\text { typischer } \\
\text { Situationen } \\
\text { (bspw. } \\
\text { Sprache) }\end{array}$ & $\begin{array}{l}\text { - Internalisierung } \\
\text { von Werten, } \\
\text { Normen, } \\
\text { Einstellungen } \\
\text { - Sprachkompetenz } \\
\text { - } \text { Mediennutzung } \\
\text { - Interesse an } \\
\text { Politik } \\
\text { - Ess- und } \\
\text { Trinkgewohn- } \\
\text { heiten } \\
\text { - Einstellung zu } \\
\text { Familie und } \\
\text { Partnerschaft } \\
\text { Bedeutung von } \\
\text { Religion }\end{array}$ & $\begin{array}{l}\text { - } \text { Anerkennung } \\
\text { von } \\
\text { Grundnormen }\end{array}$ \\
\hline
\end{tabular}

Quelle: Eigene Darstellung.

\subsection{Fragestellungen und Hypothesen}

Die Darstellung der Ansätze zu Ergebnissen und Dimensionen von Integration zeigt: Das Konzept, und damit das Verständnis von Integration, befindet sich stetig in der Entwicklung. So zeigt sich im Rahmen der Ausführungen zu Ergebnissen von Integration zwar, dass das ursprüngliche Assimilationsparadigma durch eine offenere Ausgestaltung abgelöst wurde. Dennoch unterliegen auch neuere Ausgestaltungen noch einer Kritik und vor allem die Diskussion um Assimilation und Pluralismus besteht weiter fort. Darüber hinaus wird in der Literatur oft nicht klar zwischen wünschenswerten und empirischen Ausgängen von Integration unterschieden. So werden pluralistische Modelle oft als wünschenswerte Ausgänge diskutiert, während der Ausgang der Assimilation starker Kritik ausgesetzt ist. Dass Assimilation jedoch auch einen empirischen Ausgang von Integration darstellen kann, wird dabei oft außer Acht gelassen.

In Bezug auf die Dimensionen von Integration hat sich gezeigt, dass unterschiedliche Autorinnen und Autoren zwar vergleichbare Dimensionen von Integration beschreiben, die darunter gefassten Aspekte sich jedoch unterscheiden. Auch wenn teilweise Aspekte - wie beispielsweise das Erlernen der Sprache - in allen Ausführungen auftauchen, zeigen sich doch gerade in Bezug auf die Ausführlichkeit der Differenzierung von Aspekten deutliche 
Unterschiede. An dieser Stelle stellt sich außerdem die Frage, wie die Ergebnisse und Dimensionen/Aspekte von Integration zusammenhängen. So scheint eine Angleichung, im Sinne von Assimilation, bei strukturellen Aspekten, wie gleicher rechtlicher Stellung und Teilhabechancen beispielsweise im Bereich Arbeitsmarkt, Bildung und der Besetzung bestimmter Positionen, unstrittig. In Bezug auf kulturelle Aspekte (bspw. Internalisierung von Werten, Normen, Einstellungen und Sprachgebrauch) kann die Assimilation jedoch in Frage gestellt werden (siehe dazu auch, Geißler 2005). Beispielsweise geht Esser (2001) davon aus, dass Integration nur in Form von Assimilation möglich ist, da sich strukturelle und kulturelle Integration (bspw. in Form des Erlernens der Sprache) gegenseitig bedingen und verstärken (Esser 2001: 22). Andere Autorinnen und Autoren gehen jedoch davon aus, dass eine strukturelle Integration auch unter Beibehaltung der Orientierung an der Herkunftskultur möglich ist (siehe hierzu bspw., Sen/Sauer/Halm 2001).

Darüber hinaus zeigt sich in Bezug auf die Dimensionen von Integration auch, dass mit der Zeit von der Vorstellung, Integration würde nur auf Leistung der Migrantinnen und Migranten beruhen, Abstand genommen wird. Vielmehr zeigen sich gerade in den neueren Ausgestaltungen auch Opportunitäten, die vorhanden sein sollten sowie Leistungen der aufnehmenden Gesellschaft.

Diese Entwicklungen machen deutlich, dass Ausführungen $\mathrm{zu}$ Integration immer vor bestimmten gesellschaftlichen und historischen Kontexten entstanden sind und aus diesem Grund auch einer gewissen Weiterentwicklung und Erneuerung bedürfen.

Der Abgleich mit dem subjektiven Verständnis der Akteurinnen und Akteure könnte dazu dienen, den Integrationsbegriff weiter zu klären, an den aktuellen Kontext anzupassen und das Vorhandensein einzelner Aspekte der theoretischen Ansätze in den subjektiven Sichtweisen von Akteurinnen und Akteuren empirisch zu überprüfen. Dabei bietet sich eine Befragung von Personen in Sportorganisationen an, da Sport immer wieder als eine Art „Integrationsmotor“ beschrieben wird und auch auf politischer Ebene eine wichtige Rolle für Integration spielt. Aus diesem Grund sollte gerade in diesem Bereich eine gewisse Sensibilisierung für die Thematik der Integration vorliegen. Interessant ist dabei auch, die Perspektive von Personen mit Migrationshintergrund mit aufzunehmen, da deren Vorstellung von Integration eventuell vom wissenschaftlichen Diskurs abweicht und bisher auch nicht innerhalb dieses Diskurses behandelt wurde. Ziel der vorliegenden Arbeit ist somit, die subjektiven Sichtweisen von Integration sowie die Erwartungen an Ergebnisse von Integration der Akteurinnen und Akteure in Sportorganisationen zu erfassen und vor dem Hintergrund des theoretischen Diskurses zum 
Integrationsbegriff gegenüberzustellen. Dabei sollen auch Gemeinsamkeiten und Unterschiede mit den vorgestellten theoretischen Konzepten aufgezeigt und die folgenden Fragen beantwortet werden:

- Welche subjektiven Sichtweisen von Integration (in Form von Verständnis von Integration und Einstellungen zu Integrationskonzepten/Ausgängen von Integration) haben die Akteurinnen und Akteure in Sportorganisationen?

- Welche Dimensionen und Aspekte von Integration sind ihnen dabei wichtig?

- Welche Ergebnisse von Integration werden für wünschenswert gehalten?

- Wie hängen Ergebnisse und Aspekte von Integration zusammen?

- Inwiefern variieren die Sichtweisen auf Integration in Abhängigkeit von sozialstrukturellen Faktoren und der Zusammensetzung der Mitglieder der Sportvereine?

Die aufgeworfenen Fragen werden $\mathrm{zu}$ einem Teil explorativ und zum anderen Teil hypothesengeleitet behandelt. So findet sich bei Beantwortung der Fragen zur Bedeutung von Dimensionen/Aspekten, Ergebnissen sowie deren Zusammenhänge ein exploratives Vorgehen. Dabei sollen die Sichtweisen der unterschiedlichen Akteursebenen (Vorstand und Mitglieder) sowie zwischen Personen mit und ohne Migrationshintergrund in Bezug auf Aspekte, Dimensionen, Ergebnisse und auch Adressatinnen und Adressaten von Integration unterschieden werden.

Die folgende Tabelle 8 stellt das Erkenntnisinteresse dieser Arbeit in Bezug auf Aspekte, Dimensionen, Ergebnisse sowie Adressatinnen und Adressaten von Integration nochmals als Überblick dar.

Tabelle 8: Übersicht des Erkenntnisinteresses der Arbeit

Thema

Erkenntnisinteresse

\begin{tabular}{l|l}
\hline Aspekte und Dimensionen von Integration & $\begin{array}{l}\text { Zugemessene Bedeutung einzelner } \\
\text { Aspekte und Dimensionen von } \\
\text { Integration }\end{array}$ \\
$\qquad \begin{array}{l}\text { Unterschiede in der Bewertung der } \\
\text { Bedeutung von Aspekten und } \\
\text { Dimensionen durch Vereinsvorstand und } \\
\text { Mitglieder sowie durch Personen mit und }\end{array}$ \\
\hline
\end{tabular}




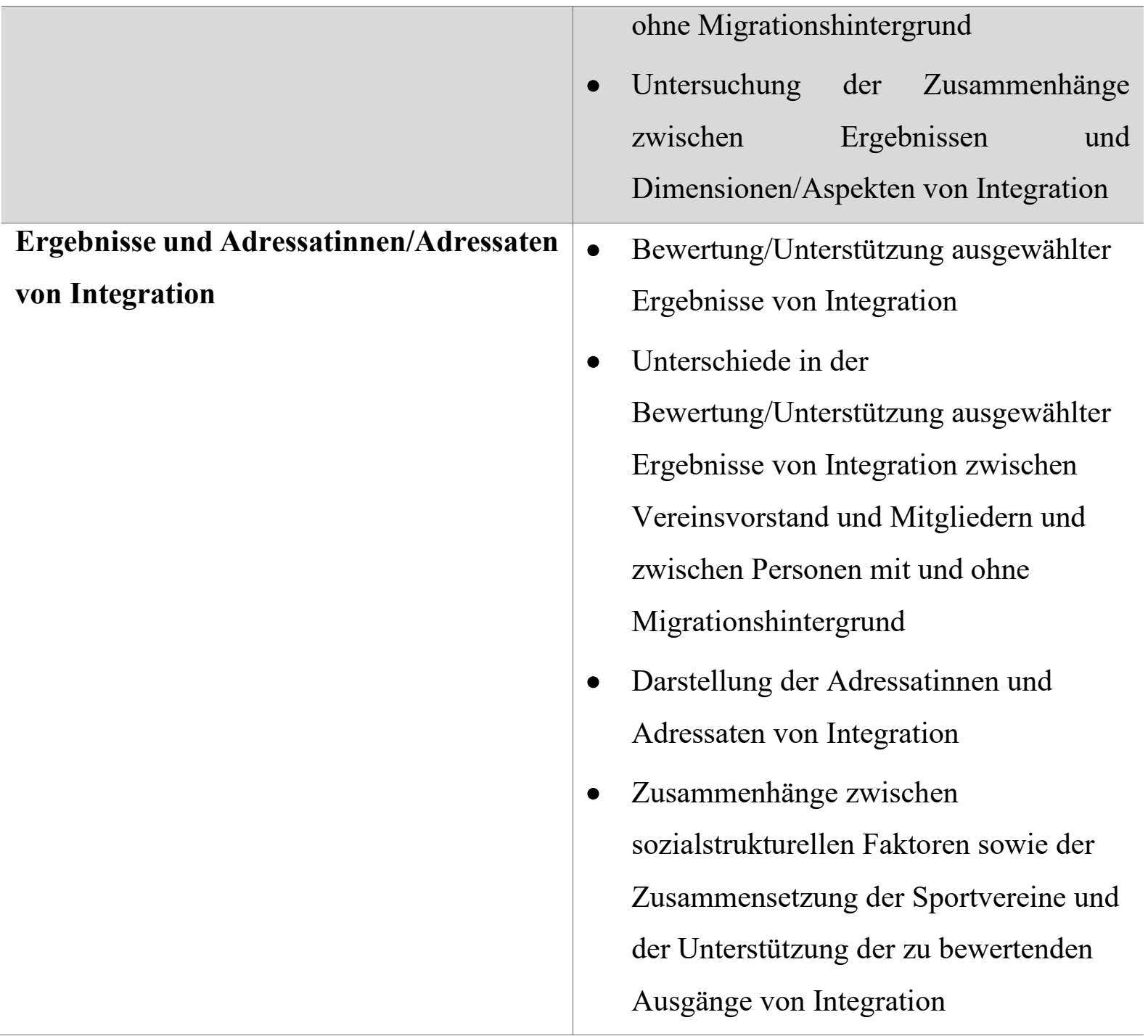

Quelle: Eigene Darstellung.

In der bisherigen dargestellten Forschung lag der Fokus eher auf der Analyse von favorisierten Integrationskonzepten in Form von Ergebnissen von Integration. Welche Aspekte und Dimensionen von Integration die Akteurinnen und Akteure jedoch für bedeutend halten und welche Adressatinnen und Adressaten die Aspekte von Integration vorrangig ansprechen, wurde in den dargestellten Arbeiten nicht behandelt. Diese Ansichten, vor allem auf Ebene des Vorstands, könnten sich jedoch in der Ausgestaltung spezieller Integrationsprogramme widerspiegeln, die von der definierten Zielgruppe dann nicht in Anspruch genommen werden, da sie nicht ihrer Integrationsvorstellung entsprechen bzw. Aspekte behandeln, die sie für Integration als eher nicht bedeutend empfinden. Beispiele hierfür finden sich in den Arbeiten von Schramkowski (2007, 2009). So wird zum einen gesagt, dass Integration oft alleinig mit dem Erwerb der Sprache gleichgesetzt wird und zum anderen wird die Ausgestaltung von Integration kritisiert. Darüber hinaus wurde in der bisherigen Forschung die Sichtweise der 
Mitglieder, zwischen denen im Verein die Interaktion vorrangig stattfindet, außer Acht gelassen (Vgl. Baur/ Mutz 2009; Strahle/Baur 2009).

Neben den Sichtweisen der unterschiedlichen Akteursebenen im Verein soll auch eine Unterscheidung der Sichtweisen zwischen Personen mit und ohne Migrationshintergrund vorgenommen werden. Im Forschungsstand hatten sich bereits Unterschiede in den Sichtweisen von Personen mit und ohne Migrationshintergrund gezeigt (Vgl. Schramkowksi 2010) und auch in Bezug auf die Favorisierung bestimmter Integrationskonzepte zeigten sich Einflüsse des Migrationshintergrundes (Vgl. Baur/ Mutz 2009). Generell existiert jedoch keine systematische Gegenüberstellung der beiden Perspektiven. Darüber hinaus konnte Baur und Mutz (2009) Zusammenhänge zwischen der Zusammensetzung der Sportgruppe nach dem Anteil an Zugewanderten und der Bewertung von Ausgängen von Integration aufzeigen.

Im Rahmen des Forschungsstandes hatte sich gezeigt, dass die beiden Ebenen Integration in den Sport und durch den Sport zu unterscheiden sind. Während die Ebene der Integration durch den Sport Kritik erfährt, zeigen sich Anhaltspunkte für eine Integration in den Sport. Darüber hinaus zeigt sich auch, dass gerade sportlich aktive Personen eine bessere Sozialintegration aufweisen. Aus diesem Grund, und da Sportorganisationen stark auf Interaktion ausgelegt sind, wird vermutet, dass gerade die Sozialintegration eine wichtige Dimension der Integration darstellt. Darüber hinaus ist die Sozialintegration voraussichtlich auch die Dimension, die von den Akteurinnen und Akteuren am direktesten wahrgenommen wird.

In Bezug auf wünschenswerte Ergebnisse von Integration zeigte sich in der vorgestellten Studie von Baur et al. (2009) eine Mischung aus assimilativen und pluralistischen Vorstellungen. Diese Untersuchung war jedoch auf Landeskoordinatorinnen und -koordinatoren sowie Ansprechpartnerinnen und -partner des Programms „Integration durch Sport“ beschränkt. Aus diesem Grund sollen in dieser Arbeit wünschenswerte Ergebnisse von Integration unter Einbezug der Mitgliederebene untersucht werden.

$\mathrm{Zu}$ der Frage der Variation der Sichtweisen auf Integration in Abhängigkeit von sozialstrukturellen Merkmalen sowie Merkmalen der Sportvereine lassen sich mit Hilfe des dargestellten Forschungsstandes mehrere Hypothesen formulieren:

- H1: Das Alter der Befragten weist einen negativen Zusammenhang mit der Unterstützung pluralistischer Konzepte auf.

- H2: Personen mit Migrationshintergrund unterstützen pluralistische Konzepte stärker als andere Konzepte. 
- H3: Umso höher der Anteil von Personen mit Migrationshintergrund in der Trainingsgruppe, desto stärker werden pluralistische Konzepte unterstützt.

- H4: Umso höher der Bildungsstand, desto stärker werden pluralistische Konzepte unterstützt.

Hypothese H1 bis H3 beziehen sich dabei auf die dargestellte Arbeit von Baur und Mutz (2009), in der ein Zusammenhang zwischen den Faktoren Alter, Migrationshintergrund und Trainingsgruppe mit der Einstellung zu Integrationskonzepten nachgewiesen werden konnte.

Die Hypothese H4 bezieht sich auf einen Zusammenhang, der in der Forschung in Bezug auf die Einstellung zu Zugewanderten immer wieder beobachtet wird. Dieser soll im Rahmen der Arbeit auf die Einstellung zu Integrationskonzepten übertragen werden. 


\section{Daten und Methoden}

Im Rahmen dieses Kapitels erfolgt zunächst eine Darstellung des Projekts „InBewegung“, bevor in den folgenden Unterkapiteln auf die quantitativen und qualitativen Erhebungs- und Auswertungsmethoden sowie die Integration der Daten eingegangen wird.

\subsection{Das Projekt „InBewegung““}

Im Folgenden werden zunächst die Ziele und Phasen des Projekts „InBewegung“ dargestellt, da dieses die Grundlage für die vorliegende Arbeit bildet. Im Anschluss wird die Methode der Mixed Methods als Forschungsdesign erläutert, da dieser Ansatz sowohl im Projekt als auch in der vorliegenden Arbeit Verwendung findet. Den Abschluss dieses Unterkapitels bilden Ausführungen zur Rekrutierung der Teilnehmenden sowie zur Grundgesamtheit und verwendeten Stichprobe.

\subsubsection{Ziele und Phasen des Projekts}

Die vorliegende Arbeit entstand im Rahmen des Projekts „InBewegung Transformationsprozesse von Sportorganisationen in der Migrationsgesellschaft“. Das Projekt wird seit Februar 2018 für eine Laufzeit von insgesamt 3 Jahren vom Bundesministerium für Bildung und Forschung gefördert (Förderkennzeichen: 01UM1804Y) und in Kooperation mit dem Sportkreis Frankfurt durchgeführt. Das Projekt „InBewegung“ untersucht in erster Linie die organisationalen Veränderungs- und Optimierungsprozesse der Vereine des Sportkreises Frankfurt vor dem Hintergrund zunehmender Diversität der Mitglieder sowie damit einhergehende Herausforderungen. Dabei soll jedoch auch ein Überblick über Integrationsbemühungen einzelner Vereine erarbeitet sowie Erwartungen und Sichtweisen der Akteurinnen und Akteure selbst betrachtet werden. Dazu werden Intentionen, Einstellungen und Handlungspraktiken der Akteurinnen und Akteure auf verschiedenen Organisationsebenen untersucht und sollen auf der Institutionsebene des Sportkreises Frankfurt schließlich in eine datenbasierte Handreichung münden.

Das Forschungsprojekt ist in mehrere Phasen gegliedert, die einzelne Teilprojekte darstellen. Diese werden in der nachfolgenden Abbildung 2 dargestellt. Das Projekt verfolgt einen MixedMethods-Ansatz, dessen Ziel die Triangulation der Ergebnisse der unterschiedlichen Teilprojekte in der Entwicklung von Empfehlungen und der partizipativen Phase darstellt. 
Abbildung 2: Phasen des Projekts in Bewegung

1. Dokumentenanalyse anhand von Homepages $(n=338)$

2. Explorative Interviews mit Vereinsvorständen $(n=8)$

3. Online-Umfrage für Vereinsvorstände und Mitglieder $(\mathrm{n}=422)$

4. Ethnografische Beobachtungen $(n=21)$

5. Online-Umfrage für außerhalb von Vereinen Aktive und Geflüchtete $(n=146)$

6. Entwicklung von Empfehlungen

7. Partizipative Phase (Workshops, Round Table, ...)

Quelle: Eigene Darstellung

Das Projekt begann mit einer Dokumentenanalyse anhand der Webseiten der Vereine. Im Rahmen dieser Dokumentenanalyse wurden 338 Vereinswebseiten mit Hilfe eines Analyserasters bezüglich angegebenen Strukturmerkmalen der Vereine sowie dem Vorhandensein von integrativen Bezügen untersucht. Im Mittelpunkt stand dabei die Identifikation von Maßnahmen zur besseren Integration von Migrantinnen und Migranten sowie von Geflüchteten in den Frankfurter Sportvereinen. Darüber hinaus entstand im Rahmen der Dokumentenanalyse eine Auswahl der Vereine, die für die weiteren Untersuchungsschritte, insbesondere die explorativen Interviews, von Interesse sein könnten.

Auf die Dokumentenanalyse folgten explorative Interviews mit acht Vereinsvorständen. Im Rahmen dieser Interviews lag der Fokus auf der Untersuchung sozialer Praktiken in Sportvereinen sowie auf der Veränderung von Vereinsstrukturen vor dem Hintergrund wachsender Heterogenität der Mitglieder. Die explorativen Interviews fanden im Vorfeld der quantitativen Befragung statt, um einen ersten Überblick über die Themen des Forschungsfeldes gewinnen zu können.

Darauf folgte eine Befragung von Vereinsvorständen und Mitgliedern $(n=422)$ mit Hilfe eines Fragebogens, ethnografische Beobachtungen $(n=21)$ und weitere fragebogenbasierte Befragungen von Geflüchteten und Personen, die außerhalb von Vereinen sportlich aktiv ( $\mathrm{n}=$ 142) sind.

Vor der eigentlichen Umfrage für Vereinsvorstände und Mitglieder wurde ein Pretest durchgeführt. Dazu wurde zum einen eine Online-Version verbreitet $(n=69)$, in der ein zusätzliches Feld für Anmerkungen vorhanden war, sowie vier face-to-face-Pretests in Form 
des Think-Alouds durchgeführt, um eventuelle Schwierigkeiten mit Formulierungen der Fragebatterie zu testen. Aufgrund von Rückmeldungen der Personen, mit denen ein face-toface-Pretest durchgeführt wurde, wurden einzelne Items umformuliert.

Die standardisierten Vorstands- und Mitgliederfragebögen umfassten mehrere Themenbereiche, um die breite des Projekts „InBewegung“ abdecken zu können. Diese Themenbereiche beinhalteten die eigene sportliche Betätigung und eine Beschreibung der Sportgruppe, Engagement im Verein, Vereinsleben und Integration sowie Angaben zur Person. Der Fragebogen für Vereinsvorstände umfasste außerdem einen Teil zu strukturellen Merkmalen des Vereins. Zur Beantwortung der Fragestellung dieser Arbeit wurde eine Fragebatterie entwickelt, die im Fragebogen innerhalb des Abschnitts zu Integration untergebracht wurde. Auf die Entwicklung dieser Fragebatterie wird im Kapitel 5.2.1 „Operationalisierung im Fragebogen“ eingegangen.

Die Befragung von Vereinsvorständen und Mitgliedern diente im Rahmen des Projekts vor allem der Analyse von organisationalen Strukturen der Vereine sowie ihrer Passung zu den Bedingungen wachsender Vielfalt. Die Befragung von Geflüchteten und Personen, die außerhalb von Vereinen sportlich aktiv sind, fokussierte im Gegensatz dazu die Hemmnisse in Bezug auf das Sporttreiben generell oder bezüglich der Mitgliedschaft in einem Verein. Im Rahmen der ethnografischen Beobachtungen sollte zudem ein Einblick in die Umsetzung von Maßnahmen im Verein sowie in Interaktionen und das soziale Miteinander gewonnen werden.

Die erhobenen Daten des Projekts fließen in die Entwicklung von Handlungsempfehlungen für den Kooperationspartner Sportkreis Frankfurt ein. Für die vorliegende Arbeit von Bedeutung sind die fragenbogenbasierten Befragungen von Vereinsvorständen und Mitgliedern in den Vereinen sowie die explorativen Interviews mit den Vereinsvorständen.

\subsubsection{Mixed Methods als Forschungsdesign}

Das Prinzip der Mixed Methods besteht aus der Einsicht, „dass sowohl qualitative als auch quantitative Methoden spezifische Begrenzungen und Probleme aufweisen, die den Rückgriff auf Verfahren aus der jeweils anderen Tradition geradezu zwingend machen“ (Baur/Kelle/Kuckartz 2017: S. 9). Die Diskussion um Mixed Methods begann dabei zu einer Zeit, die immer wieder als Zeit der „Paradigmenkriege“ bezeichnet wird. So äußerten Vertreterinnen und Vertreter quantitativer Ansätze immer wieder Zweifel in Bezug auf die Wissenschaftlichkeit qualitativer Methoden. Vertreterinnen und Vertreter qualitativer Ansätze 
zweifelten umgekehrt an quantitativen Methoden (Baur/Kelle/Kuckartz 2017: S. 7). Seit den 1980er Jahren und zahlreichen Publikationen, die den methodologischen Standpunkt von qualitativen Methoden näher erläutern, scheint dieser „Paradigmenkrieg“ durch eine „,friedliche Koexistenz [...], bei der man sich wechselseitig ignoriert“, (Baur/Kelle/Kuckartz 2017: S. 7) abgelöst worden $\mathrm{zu}$ sein. Jedoch bergen gerade die Unterschiede von quantitativen und qualitativen Methoden Potenzial für die Weiterentwicklung von Forschungsmethoden“ (Baur/Kelle/Kuckartz 2017: S. 9). Anzumerken ist hierbei auch, dass die Kombination von Methoden keineswegs ein neues Phänomen darstellt, sondern bereits in frühen Studien, wie beispielweise der Marienthal-Studie, Anwendung fand (Kelle 2017: S. 41/42).

Greene et al. (1989) unterscheiden insgesamt fünf unterschiedliche Motivationen für Mixed Methods Designs und stellen dabei auf bestimmte Aufgaben der Methodenkombination ab. So unterscheiden die Autorinnen zwischen Triangulation, Komplementarität, Entwicklung, Initiation und Expansion. Durch die Triangulation soll eine Validierung der aufgefundenen Ergebnisse durch den Einbezug einer zweiten Perspektive möglich sein, während die Komplementarität der Vervollständigung der Ergebnisse einer Methode durch den Einbezug von Ergebnissen einer anderen Methodik dient. Die Motivation der Entwicklung zielt darauf ab, die Ergebnisse einer Teilstudie zu nutzen, um die Methode der Folgestudie zu entwickeln. Dies ist beispielsweise bei der Entwicklung von Fragebogeninstrumenten sinnvoll. Im Gegensatz dazu sollen im Rahmen der Initiation Widersprüche entdeckt und durch Einsatz der Expansion soll die inhaltliche Breite und Reichweite einer Studie verbessert werden (Greene et al. 1989: S. 259).

Nach Creswell et al. (2003) sind insgesamt vier Kriterien bei der Wahl eines Mixed Methods Ansatzes zu berücksichtigen. Diese sind Implementation, Priorität, Integration und die Rolle der theoretischen Perspektive. Im Rahmen der Implementation ist zu entscheiden, ob zunächst die quantitative bzw. qualitative Studie durchgeführt wird, oder diese parallel stattfinden. Werden die Teilstudien hintereinander durchgeführt spricht man von einem sequenziellen Design (Zwei-Phasen-Design). Weiter ist festzulegen, ob einer Methodik Priorität eingeräumt wird oder deren Resultate als gleichwertig behandelt werden. Das Kriterium der Integration bezieht sich auf den Zeitpunkt, zu dem die Integration von qualitativen und quantitativen Daten stattfindet. Meistens ist dies in der Datenanalyse der Fall. Werden in einem Fragebogen jedoch auch offene Fragen verwendet, findet die Integration bereits in der Datenerhebung statt. Zuletzt ist auch zu berücksichtigen, ob dem Design ein theoretischer Rahmen zugrunde liegt (Creswell et al. 2003: S. 168-177). Aus diesen Kriterien lassen sich insgesamt 72 
Kombinationsmöglichkeiten ableiten. Creswell et al. identifizieren jedoch sechs Hauptstrategien für Mixed Methods, die jeweils drei sequenzielle und parallele Designs abbilden (Creswell et al. 2003: S. 177/178).

Die sequenziellen Verfahren umfassen dabei das vertiefende Design, das generalisierende Design und das transformative Design. Parallele Verfahren sind die parallele Triangulationsstrategie, das parallele verschachtelte Design und das transformative parallele Design. Beim vertiefenden Design folgt auf eine quantitative Erhebung eine qualitative Studie, mit Hilfe derer die Ergebnisse aus der quantitativen Studie vertieft werden, aber auch eventuell überraschende Ergebnisse weiter geklärt werden sollen. Das generalisierende Design baut hingegen auf einer qualitativen Studie auf. Dabei sollen die Ergebnisse der qualitativen Studie durch Zahlenangaben generalisiert und Zusammenhänge identifiziert werden. Im Gegensatz dazu baut das transformative Design durchgehend auf einer theoretischen Perspektive auf und unterschiedliche Perspektiven werden mit Hilfe unterschiedlicher Methoden beleuchtet.

Im Rahmen der parallelen Designs beschreibt die parallele Triangulationsstrategie ein Vorgehen, bei dem separat unter strikter Trennung quantitative und qualitative Studien durchgeführt werden, deren Resultate dann miteinander verglichen werden können. Das parallel verschachtelte Design enthält hingehen einen dominierenden Datentyp und es werden zusätzlich Daten mit Hilfe des ergänzenden Datentyps erhoben, um bestimmte Teilfragen zu beantworten. Beim transformativen parallelen Design spielt, wie beim sequenziellen transformativen Design, ein theoretischer Rahmen eine bedeutende Rolle (Creswell et al. 2003: S. 177-186).

Als Methode ist der Mixed-Methods-Ansatz auch Kritik ausgesetzt. So wird der Ansatz teilweise als Modeerscheinung abgetan oder als ein nicht wirklich neuer Ansatz gesehen. Studien zeigen, dass der Anteil an Mixed-Methods-Projekten von 1996 bis 2008 zwar stark gestiegen ist, jedoch ist der Gesamtanteil solcher Projekte immer noch gering (Kuckartz 2014: 155/156). Für diese Arbeit bedeutend sind die Kritiken an den sogenannten „Pseudo-MixedMethods“ und der Integration der Ergebnisse. Der Begriff der „Pseudo-Mixed-Methods“ bezieht sich auf die Verknüpfung quantitativer und qualitativer Teilstudien mit unterschiedlichen Fragestellungen. Es muss daher bei der Anwendung eines Mixed-MethodsDesigns darauf geachtet werden, dass in beiden methodischen Ansätzen die gleiche Fragestellung behandelt wird (Kuckartz 2014: 157). Dies ist in der vorliegenden Arbeit der Fall, da sowohl in der quantitativen Befragung als auch in den Interviews, die Sichtweisen auf Integration fokussiert werden. Ein weiterer wichtiger Kritikpunkt an Mixed-Methods-Projekten 
bezieht sich auf die Integration der Ergebnisse. So kommt es in manchen Studien vor, dass die Ergebnisse der beiden Teilstudien eher nebeneinander aufgelistet werden, als dass es zu einer wirklichen Verbindung kommt (Kuckartz 2014: 157). Auf das Vorgehen zur Integration der Daten innerhalb dieser Arbeit wird in Kapitel 5.4 „Integration der Daten“ eingegangen.

\subsubsection{Grundgesamtheit und Stichprobe}

Die Grundgesamtheit, die der Erhebung zugrunde liegt, umfasst alle Vereine und Vereinsmitglieder, die dem Sportkreis Frankfurt angehören. Dieser umfasste zum Zeitpunkt der Erhebung 419 Vereine mit etwas mehr als 237.000 Mitgliedern.

Die quantitative Befragung für Vereinsvorstände und Mitglieder wurde zunächst als reine Online-Umfrage konzipiert und die 419 Vereine des Sportkreises Frankfurt per Mail um ihre Unterstützung gebeten. Diese Unterstützung umfasste zum einen das Ausfüllen der Vorstandsbefragung durch eine Person des Vereinsvorstands sowie die Weiterleitung der Mitgliederbefragung an die Vereinsmitglieder. Dieses Vorgehen wurde gewählt, da eine andere Art der Kontaktierung der Vereinsmitglieder aufgrund datenschutzrechtlicher Einschränkungen nicht möglich war.

Die Weiterleitung an die Mitglieder war nicht in allen Vereinen möglich, da gerade kleine Vereine nicht über E-Mail-Listen ihrer Mitglieder verfügen. Aufgrund dessen und des anfänglich geringen Rücklaufs, wurden in einem nächsten Schritt Flyer mit einem QR-Code, der zur Mitgliederumfrage führt, an 80 zufällig ausgewählte Vereine versendet. Zusätzlich wurde eine Papierversion des Mitgliederfragebogens erstellt und die Befragung in zufällig ausgewählten Vereinen vor Ort durchgeführt. Außerdem wurde auf den Webseiten des Sportkreises Frankfurt und Mainova-Sport für die Umfrage geworben. Diese Maßnahmen konnten den Rücklauf, in Kombination mit zwei erneuten Kontaktaufnahmen per Mail und Anrufen, steigern.

Die Auswahl der Vereinsvorstände für die explorativen Interviews erfolgte auf Basis der zu Beginn des Projekts durchgeführten Dokumentenanalyse. Diese Analyse, die anhand der Webseiten der Vereine durchgeführt wurde, generierte Informationen über vorliegende Integrationsbemühungen der Vereine. Für die explorativen Interviews wurden dann Vereine ausgewählt, die bereits im Bereich der Integration aktiv sind bzw. das Thema Integration zumindest auf ihrer Webseite behandeln. Von besonderem Interesse waren dabei Vereine, die 
als Stützpunktvereine des Programms „Integration durch Sport“ begleitet und zum Teil auch finanziell unterstützt werden.

Für die vertiefenden Interviews mit Vereinsmitgliedern erfolgte eine zufällige Auswahl der Teilnehmerinnen und Teilnehmer. Hierzu fand am Ende der Mitgliederbefragung eine Weiterleitung auf eine Abfrage zur Bereitschaft zur Teilnahme an einem Interview statt. Diese Daten wurden getrennt von der eigentlichen Mitgliederbefragung erhoben und gespeichert.

Im Rahmen der quantitativen Befragung von Vereinsvorständen und Mitgliedern konnten 422 Befragungsteilnehmerinnen und -teilnehmer verzeichnet werden. Dies entspricht, bezogen auf alle Mitglieder des Sportkreises Frankfurt, einer Teilnahmequote von 0,18 \%. Insgesamt konnten somit, trotz umfangreicher Aktionen zur Nacherfassung von Befragten, nur wenige Mitglieder erreicht werden, weshalb eine Verallgemeinerung der Ergebnisse zu vermeiden ist. Die Befragten gliedern sich in 85 Vorstände und 337 Mitglieder. Insgesamt wurden 344 Personen ohne Migrationshintergrund und 78 mit Migrationshintergrund befragt. Die Personen mit Migrationshintergrund gliedern sich wiederrum in 30 Migrantinnen und Migranten mit eigener Migrationserfahrung, die somit der ersten Generation angehören, und 48 Migrantinnen und Migranten zweiter Generation ohne eigene Migrationserfahrung. Außerdem konnten Personen mit europäischem und außereuropäischem Migrationshintergrund unterschieden werden.

Die qualitativen Interviews umfassen insgesamt acht Interviews mit ausgewählten Vereinsvorständen sowie fünf vertiefende Interviews mit Vereinsmitgliedern. Die Interviews mit Vereinsmitgliedern wurden unabhängig vom Projekt „InBewegung“ durchgeführt, um einzelne Aspekte der quantitativen Ergebnisse zu ergänzen bzw. zu erweitern. Für die vorliegende Fragestellung wurden dafür fünf zielgerichtete kurze Leitfaden-Interviews mit Vereinsmitgliedern realisiert. Bei diesen vertiefenden Interviews fand eine strengere Orientierung am Leitfaden statt. Außerdem wurden im Rahmen dieser Interviews Personen mit und ohne Migrationshintergrund miteinbezogen. Insgesamt zwei der interviewten Personen weisen eine eigene Migrationserfahrung auf.

Bei Betrachtung der quantitativen Stichprobe zeigt sich, dass das Alter und der Bildungsstand der Befragten eine schiefe Verteilung aufweisen. Darüber hinaus liegen auch fehlende Werte vor. Aufgrund der generell niedrigen Fallzahl wird in den Analysen von einem listenweisen Fallausschluss abgesehen, da dieser zu einer weiteren Verkleinerung der Stichprobe führen würde (Wirtz 2004: 112). Aus diesem Grund finden auch keine anderen Verfahren, wie die 
Ersetzung durch den Mittelwert und Imputationen ${ }^{17}$, Anwendung. Demnach wird in der vorliegenden Arbeit mit einem paarweisen Fallausschluss gearbeitet. Dabei werden für die Berechnungen alle verfügbaren Informationen verwendet. Dies kann dazu führen, dass den Berechnungen unterschiedliche Teilstichproben zugrunde liegen, was im Rahmen der Limitationen beachtet werden sollte (Wirtz 2004: 113).

Die Auswertung der Altersvariable zeigt, dass hauptsächlich Personen im Alter zwischen 21 und 30 Jahren (22,6\%) an der Befragung teilgenommen haben (vgl. Abbildung 3). Insgesamt zeigt sich hier eine rechtsschiefe Verteilung. Diese Verteilung ist keineswegs spezifisch für die Sportvereine, da die meisten Personen in Sportvereinen 2019 zwischen 41 und 60 Jahren alt waren (25,7 \%). Bezogen auf ihren Anteil an der Bevölkerung wiesen jedoch die 7- bis 14Jährigen den höchsten Organisationsgrad auf (70,5 \%). Darauf folgen Personen zwischen 15 und 18 Jahren $(55,4 \%)^{18}$ (DOSB 2020).

Abbildung 3: Verteilung der Variable Alter in Prozent

Alter der Befragten $(\mathrm{n}=366)$

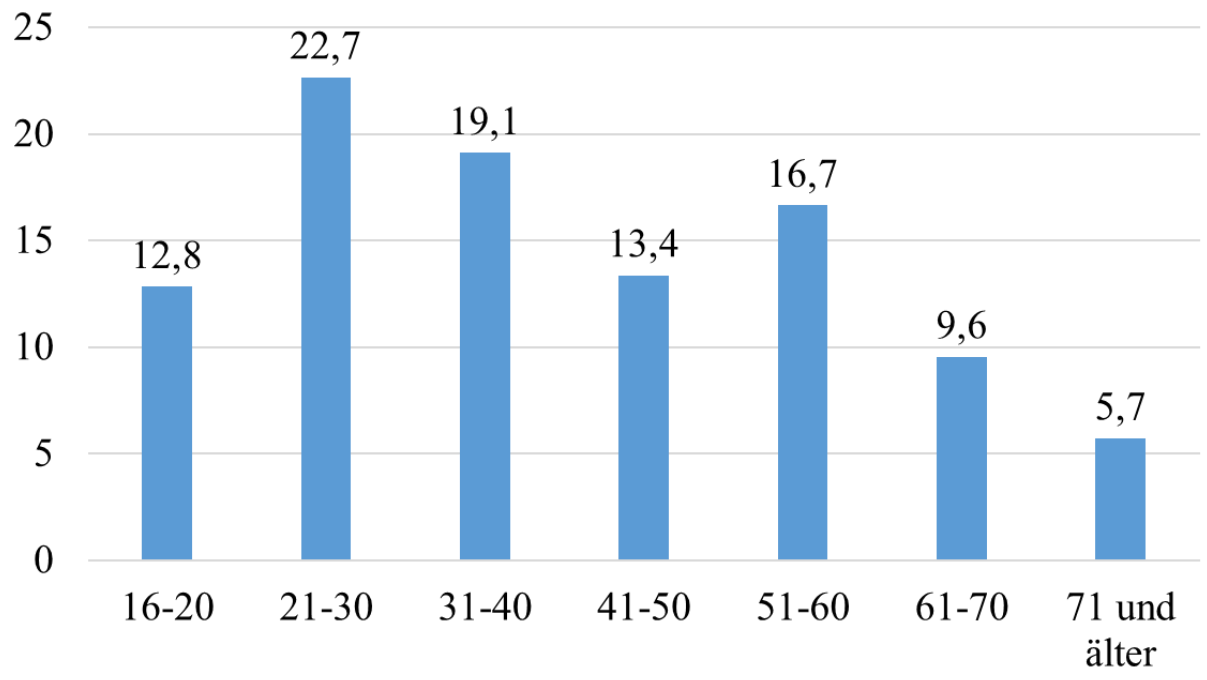

Quelle: Eigene Darstellung.

Betrachtet man die Verteilung der Variable zum Bildungsstand, zeigt sich, dass in der Stichprobe vor allem Personen mit Abitur vorhanden sind (72,2\%) (vgl. Abbildung 4). Dadurch ergibt sich auch hier eine schiefe Verteilung der Variable.

\footnotetext{
${ }^{17}$ Hierdurch könnte es außerdem zu einer Verzerrung der „wahren“ Verteilungen kommen.

${ }^{18}$ Siehe Anhang für eine ausführliche Tabelle zu Anzahl der Mitgliedschaften und dem Organisationsgrad in Sportvereinen nach Alter.
} 
Abbildung 4: Verteilung der Variable Bildungsstand in Prozent

Bildungsstand $(\mathrm{n}=388)$

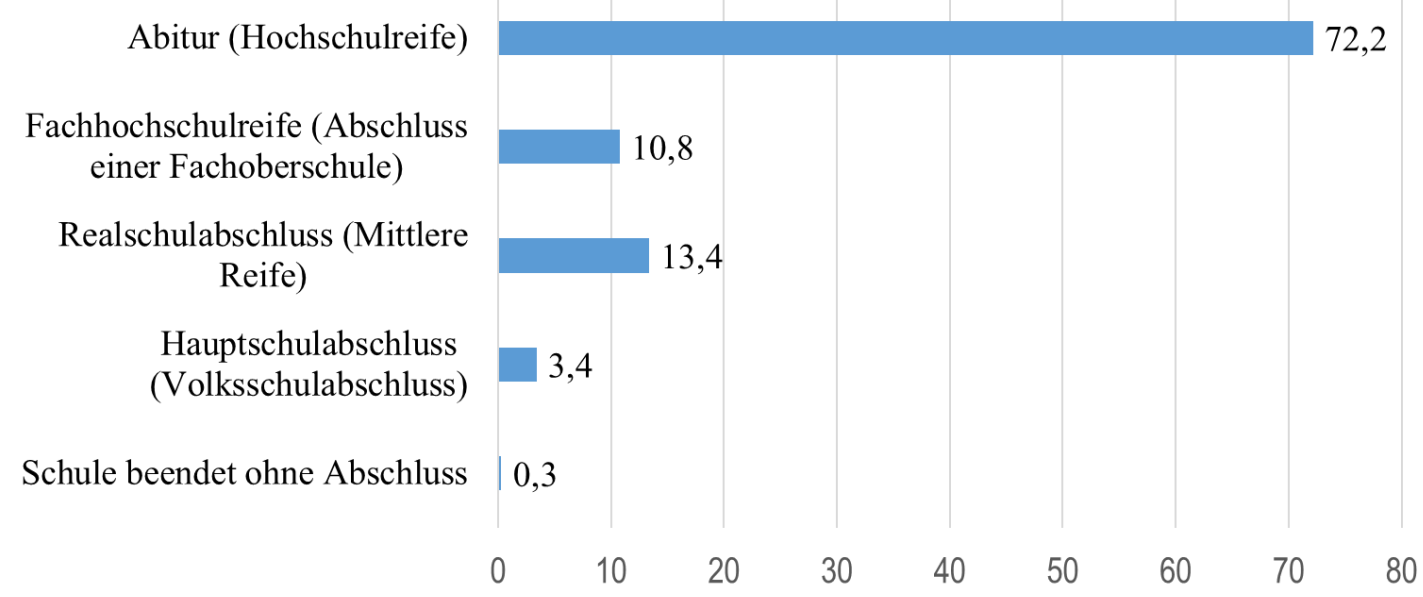

Quelle: Eigene Darstellung.

In Bezug auf die genannten Faktoren zeigen sich jedoch keine signifikanten Unterschiede in der Verteilung zwischen Personen mit und ohne Migrationshintergrund in der Stichprobe ${ }^{19}$, was die Vermutung nahelegt, dass auch Personen mit Migrationshintergrund innerhalb der Stichprobe sehr selektiv sind.

Die Zusammensetzung der Stichprobe, hinsichtlich des Bildungsstands, könnte mit Limitationen im Rahmen der Auswertungen verbunden sein. Da auch innerhalb der Hypothesen angenommen wird, dass Personen mit einem höheren Bildungsstand Integration eher positiv gegenüberstehen bzw. pluralistische Konzepte unterstützen. Dies könnte zu einer überwiegend positiven Bewertung in der Befragung führen. Jedoch kann diese Zusammensetzung der Stichprobe auch der vermehrten Partizipation von Personen mit höherer Bildung in Sportvereinen geschuldet sein, wodurch diese Zusammensetzung als spezifisch für Sportvereine anzusehen ist. So zeigen beispielsweise Mutz und Burrmann (2015), dass die Mitgliedschaft in Sportvereinen einer gewissen Selektivität unterliegt und die Mitgliedschaftsquote mit höherer Bildung zunimmt (Mutz/Burrmann 2015: 86).

Im Rahmen der Ergebnisse sollten somit eventuell vorliegende Selektionsmechanismen der Befragungsteilnehmerinnen und -teilnehmer reflektiert werden. Zum einen hat sich in der vorangegangenen Darstellung von Einzelitems gezeigt, dass die Stichprobe überwiegend Personen mit einem hohen Bildungsstand enthält. Einhergehend mit den aufgeworfenen

\footnotetext{
${ }^{19}$ Alter $\chi^{2}(6)=9,31 ; \mathrm{p}=0,156$

Bildungsstand $\chi^{2}(4)=2,97 ; \mathrm{p}=0,563$
} 
Hypothesen, dass dieses Merkmal zu einer höheren Unterstützung von pluralistischen Integrationskonzepten führt, könnte auch die Einstellung zu Integration im Generellen sowie die Bewertung einzelner Aspekte positiver ausfallen.

Zum anderen könnte die Umfrage aufgrund des deutlichen Bezugs zu Integration auch überwiegend von Personen ausgefüllt worden sein, die eine positive oder offener Einstellung zum Thema Integration aufweisen bzw. diesem Thema zumindest offen gegenüberstehen.

\subsection{Quantitative Methoden}

Im Rahmen dieses Unterkapitels soll näher auf die vorgenommenen Operationalisierungen und verwendeten Auswertungsmethoden für die vorliegende Arbeit eingegangen werden. Dazu erfolgt zunächst eine Beschreibung der Operationalisierung der Items für die Fragebatterie zur Bewertung von Aspekten und Ergebnissen von Integration sowie darauffolgend eine Erläuterung der Analysemethoden für die quantitativen Daten.

\subsubsection{Operationalisierung im Fragebogen}

Im Folgenden werden die Operationalisierungen zur wahrgenommenen Bedeutung verschiedener Aspekte von Integration, der Einstellung zu verschiedenen Ausgängen von Integration sowie die Operationalisierungen verschiedener sozialstruktureller Merkmale der Befragten und Merkmalen der Sportvereine vorgestellt.

\subsubsection{Wahrgenommene Bedeutung verschiedener Aspekte für Integration}

Die Darstellungen im theoretischen Rahmen dieser Arbeit haben bereits gezeigt, dass Integration nicht nur Anforderungen an Migrantinnen und Migranten stellt, sondern dass auch Anforderungen an die Gesellschaft gestellt werden und Opportunitäten in der Gesellschaft vorhanden sein müssen, damit Integration gelingen kann. Aus den dargestellten Ansätzen wurden für die vorliegende Arbeit insgesamt dreizehn Aspekte der Integration ausgewählt. Bei dieser Auswahl wurde zum einen berücksichtigt, dass alle vier Dimensionen von Integration vorhanden sind. Zum anderen sollten auch alle der beschriebenen Adressatenebenen in der Fragebatterie $\mathrm{zu}$ finden sein sowie eine Orientierung an acht identifizierten Oberbegriffen vorgenommen werden. Darüber hinaus wurden Aspekte berücksichtigt, die sich in einem 
Großteil der Ansätze finden und gleichzeitig auch Aspekte, die nur in einzelnen Ansätzen beachtet werden.

Im Folgenden wird die Operationalisierung der Fragebogenitems anhand der acht identifizierten Oberkategorien dargestellt. Diese Kategorien umfassen:

- Aneignung von Wissen und Werten

- Kontakte

- Zugehörigkeit/Identifikation

- Partizipation

- Akzeptanz/Anerkennung

- Zufriedenheit

- Transformation

- Stellung in der Gesellschaft

In Bezug auf die Oberkategorie „Aneignung von Wissen und Werten“ wurden die beiden Aspekte ausgewählt, denen innerhalb der diskutierten Ansätze (vgl. Tabelle 9) und auch im medialen sowie gesellschaftlichen Diskurs die meiste Aufmerksamkeit zukommt. Diese Aspekte sind die Beherrschung der Sprache und die Anerkennung von Werten bzw. Grundnormen.

Tabelle 9: Zusammenfassung der Aspekte zum Oberbegriff Aneignung von Wissen und Werten

Eisenstadt

Hoffmann- Esser Nowotny

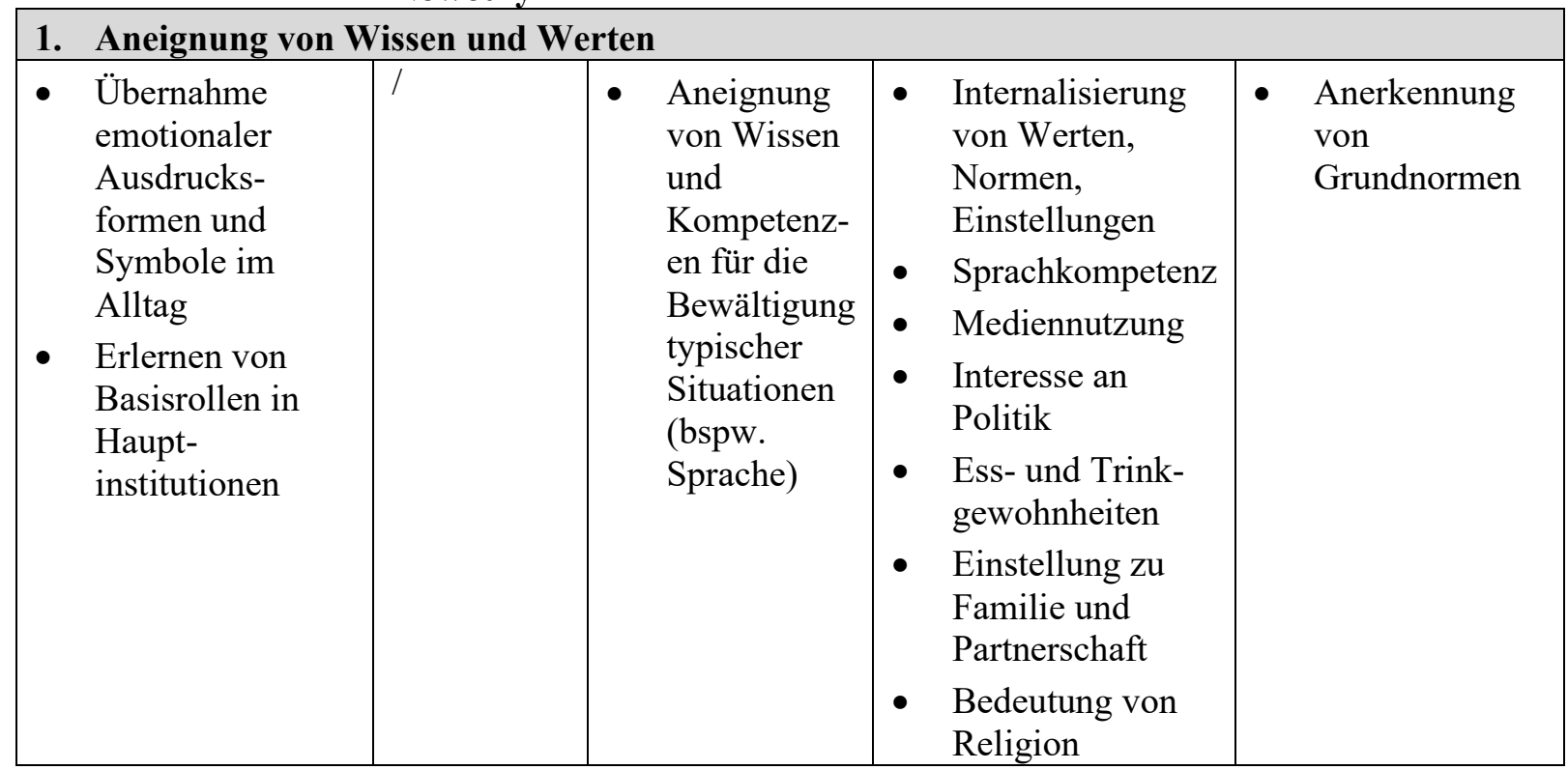

Quelle: Eigene Darstellung. 
Nach diesem Vorgehen wurden auch die anderen Oberbegriffe operationalisiert. In Bezug auf die Kontakte wurde das Item „Kontakte zwischen Menschen unterschiedlicher Kulturen“ gewählt, da der Fokus in den Beschreibungen dieser Interaktion gerade auf den Kontakten zwischen diesen Gruppen liegt (vgl. Tabelle 10). Eisenstadt (1951; 1952a; 1952b) und Hoffmann-Nowotny $(1973 ; 1990)$ beschreiben diese Kontakte eher unspezifisch als soziale Interaktion. Esser (2001) und Heckmann et al. (2000) verwenden die Bezeichnung der „,interethnischen Kontakte“, welche hier jedoch als zu komplex angenommen wurde. Der Fokus bei Anhut und Heitmeyer (2009), der auf der emotionalen Ebene anzusiedeln ist, wird im Rahmen der Befragung nicht aufgenommen, da dieser als $\mathrm{zu}$ abstrakt und schwer operationalisierbar wahrgenommen wird und die Aspekte emotionale und expressive Beziehungen sowie die Sicherung des sozio-emotionalen Rückhalts mehrere Ebenen beinhalten, die in der geplanten Kürze der Fragebatterie nicht aufführbar sind.

Tabelle 10: Zusammenfassung der Aspekte zum Oberbegriff Kontakte

\begin{tabular}{|c|c|c|c|c|}
\hline Eisenstadt & $\begin{array}{l}\text { Hoffmann- } \\
\text { Nowotny }\end{array}$ & Esser & Heckmann & Anhut/Heitmeyer \\
\hline 2. Kontakte & & & & \\
\hline $\begin{array}{l}\text { - Aufbau } \\
\text { sozialer } \\
\text { Kontakte }\end{array}$ & $\begin{array}{ll}\text { - } & \text { Soziale } \\
\text { Interaktion }\end{array}$ & $\begin{array}{l}\text { Aufbau } \\
\text { interethnischer } \\
\text { Kontakte } \\
\text { - Gelegenheiten } \\
\text { des Zusammen- } \\
\text { treffens } \\
\text { Eröffnung } \\
\text { sozialer } \\
\text { Gelegenheiten }\end{array}$ & $\begin{array}{l}\text { - Interethnische } \\
\text { Kontakte } \\
\text { (Freundschaften, } \\
\text { Partnerschaften) }\end{array}$ & $\begin{array}{l}\text { Emotionale und } \\
\text { expressive } \\
\text { Beziehungen } \\
\text { - Sicherung } \\
\text { sozio- } \\
\text { emotionalen } \\
\text { Rückhalts }\end{array}$ \\
\hline
\end{tabular}

Quelle: Eigene Darstellung.

Bei Hoffmann-Nowotny (1973; 1990) sowie Anhut und Heitmeyer (2009) zeigen sich keine Ausführungen zum Oberbegriff der Zugehörigkeit/Identifikation (vgl. Tabelle 11). Die Aspekte, die im Rahmen der anderen Konzepte unter diesem Oberbegriff aufgeführt werden können, zeigen zwar unterschiedliche Ausgestaltungen, diese beziehen sich jedoch im Grunde, wie im Item formuliert, auf das „Vorhandensein eines Zugehörigkeitsgefühls“. 
Tabelle 11: Zusammenfassung der Aspekte zum Oberbegriff Zugehörigkeit/Identifikation

\begin{tabular}{|c|c|c|c|c|}
\hline Eisenstadt & $\begin{array}{l}\text { Hoffmann- } \\
\text { Nowotny }\end{array}$ & Esser & Heckmann & $\begin{array}{l}\text { Anhut/ } \\
\text { Heitmeyer }\end{array}$ \\
\hline \multicolumn{5}{|c|}{ 3. Zugehörigkeit/Identifikation } \\
\hline $\begin{array}{l}\text { - Positive Identifikation } \\
\text { mit Werten und } \\
\text { Strukturen } \\
\text { - } \\
\text { Identifikation und } \\
\text { Solidarisierung mit } \\
\text { Wertvorstellungen } \\
\text { - Zugehörigkeitsgefühl }\end{array}$ & 1 & $\begin{array}{ll}\text { - } & \text { Kollektive } \\
\text { Orientierung } \\
\text { - } & \text { Bürgersinn } \\
\text { - } & \text { Hinnahme }\end{array}$ & $\begin{array}{l}\text { - Zugehörigkeits- } \\
\text { und } \\
\text { Heimatgefühle } \\
\text { - Rückkehrabsicht } \\
\text { • } \\
\text { Bezug zum } \\
\text { „Heimatland“ }\end{array}$ & l \\
\hline
\end{tabular}

Quelle: Eigene Darstellung.

Betrachtet man die Aspekte, die sich auf Partizipation beziehen (vgl. Tabelle 12), zeigt sich zunächst, dass es unter dieser Oberkategorie im Vergleich zu den drei zuvor behandelten Kategorien wesentlich weniger Nennungen gibt. Neben einer unspezifischen Nennung als „aktive Partizipation“ bei Eisenstadt (1953), werden hier die „Partizipation in Organisationen“ bei Heckmann et al. (2000) und „Teilnahmechancen“ und „Teilnahmebereitschaft“ bei Anhut und Heitmeyer (2009) genannt. Da hierbei unterschiedliche Ebenen von Akteurinnen und Akteuren sowie unterschiedliche Bezugssysteme für die Partizipation von Bedeutung sind, werden alle drei Ausführungen als „Mitgliedschaft/Engagement in Organisationen“, „Chancen zur Teilnahme an der Gesellschaft“ und „Der Wille, an der Gesellschaft teilzunehmen“ in die Fragebatterie aufgenommen.

Tabelle 12: Zusammenfassung der Aspekte zum Oberbegriff Partizipation
Eisenstadt
Hoffmann- Esser
Heckmann
Anhut/Heitmeyer

\begin{tabular}{|c|c|c|c|c|c|}
\hline \multicolumn{6}{|c|}{ Nowotny } \\
\hline 4. & Partizipation & & & & \\
\hline & $\begin{array}{l}\text { Aktive } \\
\text { Partizipation }\end{array}$ & l & l & $\begin{array}{l}\text { - } \text { Partizipation in } \\
\text { Organisationen }\end{array}$ & $\begin{array}{ll}\text { - } & \text { Teilnahmechancen } \\
\text { - } & \text { Teilnahmebereitschaft }\end{array}$ \\
\hline
\end{tabular}

Quelle: Eigene Darstellung.

Auch in Bezug auf die Oberkategorien Akzeptanz/Anerkennung und Zufriedenheit zeigen sich nur vereinzelte Aspekte bei Anhut und Heitmeyer (2009) (vgl. Tabelle 13). Gerade da diese Aspekte nur in einem der Konzepte auftauchen, sollen diese mit in die Befragung eingehen, um zu überprüfen, als wie bedeutsam für Integration diese eingeschätzt werden. Im Rahmen der Akzeptanz/Anerkennung erfolgt eine Operationalisierung als „Anerkennung durch Andere“, da die durch die Autoren verwendeten Bezeichnungen als zu komplex für Befragungspersonen, die sich nicht mit dem wissenschaftlichen Diskurs auseinandersetzen, wahrgenommen werden. Im Gegensatz dazu wird die Formulierung der Zufriedenheit als „Zufriedenheit mit beruflicher und sozialer Stellung“, wie in der Tabelle aufgeführt, übernommen. 
Tabelle 13: Zusammenfassung der Aspekte zu den Oberbegriffen Akzeptanz/Anerkennung und Zufriedenheit

\begin{tabular}{|c|c|c|c|c|}
\hline Eisenstadt & $\begin{array}{l}\text { Hoffmann- } \\
\text { Nowotny }\end{array}$ & Esser & Heckmann & Anhut/Heitmeyer \\
\hline \multicolumn{5}{|c|}{ 5. Akzeptanz/Anerkennung } \\
\hline l & / & 1 & 1 & $\begin{array}{l}\text { - Ausgleich konfligierender Interessen } \\
\text { - Anerkennung durch Kollektiv und soziale } \\
\text { Umwelt } \\
\text { - Akzeptanz kollektiver Identitäten und } \\
\text { deren Symbole durch Andere } \\
\end{array}$ \\
\hline \multicolumn{5}{|c|}{ 6. Zufriedenheit } \\
\hline l & 1 & 1 & 1 & $\begin{array}{l}\text { - Zufriedenheit mit beruflicher und sozialer } \\
\text { Stellung }\end{array}$ \\
\hline
\end{tabular}

Quelle: Eigene Darstellung.

Im Bereich der Transformation führen Heckmann et al. (2000) aus, dass auch die aufnehmende Gesellschaft sich verändert. Dies ist aber eher als ein Ergebnis von Integration zu sehen und wird aus diesem Grund nicht in die Fragebatterie zu Aspekten von Integration aufgenommen. Darüber hinaus kann dieser beidseitige Charakter von Integration durch die Bedeutung der unterschiedlichen Ebenen von Akteurinnen und Akteuren abgebildet werden.

Der letzte Oberbegriff bildet die Stellung in der Gesellschaft (vgl. Tabelle 14). Hier werden erneut von allen Autorinnen und Autoren Aspekte aufgeführt. Von besonderer Bedeutung sind hier die rechtliche Stellung, der Bildungsstand, die berufliche Stellung sowie die Wohnsituation bzw. der Zugang zum Wohnungsmarkt, da diese Aspekte in den meisten Konzepten aufgeführt werden. Diese vier Aspekte gehen ebenfalls als Einzelitems in die Fragebatterie ein. 
Tabelle 14: Zusammenfassung der Aspekte zum Oberbegriff Stellung in der Gesellschaft

\begin{tabular}{|c|c|c|c|c|}
\hline Eisenstadt & $\begin{array}{l}\text { Hoffmann- } \\
\text { Nowotny }\end{array}$ & Esser & Heckmann & Anhut/Heitmeyer \\
\hline \multicolumn{5}{|c|}{ 7. Stellung in der Gesellschaft } \\
\hline $\begin{array}{l}\text { - Übernahme von } \\
\text { Rollen im } \\
\text { Wirtschafts- } \\
\text { leben }\end{array}$ & $\begin{array}{ll}\text { - } & \text { Rechtliche } \\
\text { - Stellung } \\
\text { - Bildung } \\
\text { - Berufliche } \\
\text { - Stellung } \\
\text { - Einkom- } \\
\text { - } \text { Won } \\
\text { - Wohnen }\end{array}$ & $\begin{array}{ll}\text { - } & \text { Rechtliche } \\
\text { Stellung } \\
\text { - } & \text { Bildung } \\
\text { - } & \text { Berufliche } \\
\text { Position } \\
\text { - } & \text { Besetzung } \\
\text { einer } \\
\text { bestimmten } \\
\text { Position }\end{array}$ & $\begin{array}{l}\text { Rechtliche } \\
\text { Stellung, } \\
\text { arbeits- und } \\
\text { sozialrecht- } \\
\text { liche Gleich- } \\
\text { stellung } \\
\text { - Bildungs- und } \\
\text { Qualifikation- } \\
\text { system } \\
\text { - Arbeitsmarkt } \\
\text { - Wohnungs- } \\
\text { markt } \\
\text { - Stellung } \\
\text { politische } \\
\text { Gemeinschaft }\end{array}$ & $\begin{array}{l}\text { - Teilhabe an } \\
\text { materiellen und } \\
\text { kulturellen } \\
\text { Gütern } \\
\text { - Zugang zum } \\
\text { Arbeitsmarkt } \\
\text { - Zugang zum } \\
\text { Wohnungs- } \\
\text { markt } \\
\text { - Zugang zum } \\
\text { Konsummarkt }\end{array}$ \\
\hline
\end{tabular}

Quelle: Eigene Darstellung.

In der nachfolgenden Tabelle 15 sind die einzelnen Aspekte, differenziert nach Oberbegriff, Dimension und Akteursebenen nochmals zusammengefasst. Die Items konnten anhand einer 5er-Skala von „Unwichtig“ bis „Sehr wichtig“ bewertet werden. Um den Begriff der Integration von der Ebene der Sportvereine zu lösen und allgemeine Einstellungen zu Integration zu messen, wurde folgende Fragestellung gewählt: „Die folgenden Fragen beziehen sich auf Ihre Einstellung zu Integration im Allgemeinen. Wie wichtig sind für Sie folgende Aspekte von Integration im Allgemeinen, also abseits vom Sport?“.

Tabelle 15: Verwendete Items zur Messung der wahrgenommenen Bedeutung verschiedener Aspekte von Integration

\begin{tabular}{l|l|l|l} 
Item & Oberbegriff & $\begin{array}{l}\text { Dimension } \\
\text { Integration }\end{array}$ & Akteurin/Akteur \\
\hline Gute Deutschkenntnisse & Werte und Wissen & Kulturation & $\begin{array}{l}\text { Anforderung an } \\
\text { Migrantinnen und } \\
\text { Migranten }\end{array}$ \\
\hline $\begin{array}{l}\text { Anerkennung wichtiger } \\
\text { Werte }\end{array}$ & Werte und Wissen & Kulturation & $\begin{array}{l}\text { Anforderung an } \\
\text { Migrantinnen und } \\
\text { Migranten }\end{array}$ \\
\hline $\begin{array}{l}\text { Chancen zur Teilnahme an } \\
\text { der Gesellschaft }\end{array}$ & Partizipation & Interaktion & Opportunitäten \\
\hline $\begin{array}{l}\text { Der Wille, an der } \\
\text { Gesellschaft teilzunehmen }\end{array}$ & Partizipation & Interaktion & $\begin{array}{l}\text { Anforderung an } \\
\text { Migrantinnen und } \\
\text { Migranten }\end{array}$ \\
\hline
\end{tabular}




\begin{tabular}{|c|c|c|c|}
\hline $\begin{array}{lr}\text { Kontakte } & \text { zwischen } \\
\text { Menschen } & \text { verschiedener } \\
\text { Kulturen } & \end{array}$ & Kontakte & Interaktion & $\begin{array}{l}\text { Anforderung an } \\
\text { Migrantinnen und } \\
\text { Migranten, } \\
\text { Opportunitäten, } \\
\text { Anforderungen an } \\
\text { Gesellschaft }\end{array}$ \\
\hline $\begin{array}{l}\text { Mitgliedschaft/Engagement } \\
\text { in Organisationen }\end{array}$ & Partizipation & Interaktion & $\begin{array}{l}\frac{\text { Anforderung an }}{\text { Migrantinnen und }} \\
\text { Migranten, } \\
\text { Opportunitäten }\end{array}$ \\
\hline $\begin{array}{l}\text { Anerkennung durch } \\
\text { Andere }\end{array}$ & Akzeptanz/Anerkennung & Interaktion & $\begin{array}{l}\text { Anforderungen an } \\
\text { Gesellschaft }\end{array}$ \\
\hline $\begin{array}{l}\text { Vorhandensein eines } \\
\text { Zugehörigkeitsgefühls }\end{array}$ & Zugehörigkeit/Identifikation & Identifikation & $\begin{array}{l}\text { Anforderung an } \\
\text { Migrantinnen und } \\
\text { Migranten }\end{array}$ \\
\hline Gleiche Rechte für Alle & Stellung in der Gesellschaft & Platzierung & Opportunitäten \\
\hline Bildungsstand & Stellung in der Gesellschaft & Platzierung & $\begin{array}{l}\text { Anforderungen an } \\
\text { Migrantinnen und } \\
\text { Migranten, } \\
\text { Opportunitäten }\end{array}$ \\
\hline Berufliche Stellung & Stellung in der Gesellschaft & Platzierung & $\begin{array}{l}\text { Anforderungen an } \\
\text { Migrantinnen und } \\
\text { Migranten, } \\
\text { Opportunitäten }\end{array}$ \\
\hline $\begin{array}{l}\text { Zugang } \\
\text { Wohnungsmarkt }\end{array}$ & Stellung in der Gesellschaft & Platzierung & Opportunitäten \\
\hline $\begin{array}{l}\text { Zufriedenheit rr } \\
\text { beruflicher und sozialer } \\
\text { Situation }\end{array}$ & Zufriedenheit & Platzierung & $\begin{array}{l}\text { Anforderung an } \\
\text { Migrantinnen und } \\
\text { Migranten }\end{array}$ \\
\hline
\end{tabular}

Quelle: Eigene Darstellung.

Die Items sollten nicht direkt auf eine zu integrierende Gruppe gerichtet sein, weshalb sich keine Formulierungen wie beispielsweise „Mitgliedschaft/Engagement von Migrantinnen oder Migranten/Zugewanderten/Personen mit Migrationshintergrund in Organisationen“ oder Ähnliches in der Fragebatterie befinden. Außerdem hätte eine solche Zuspitzung der Items zu Problemen bei der Beantwortung durch Personen mit Migrationshintergrund führen können. Die aus den theoretischen Konzepten abgeleiteten Aspekte von Integration sind aus diesem Grund so neutral wie möglich gehalten. Dies führt dazu, dass gerade die Ebene der Akteurinnen und Akteure nicht immer trennscharf festzulegen ist. So kann beispielsweise der Bildungsstand eine Anforderung an Migrantinnen und Migranten oder Opportunitäten in der Gesellschaft, überhaupt eine gewisse Bildung erreichen zu können, darstellen. Darüber hinaus sind auch Items wie „Wichtige Werte anerkennen“ unklar, da nicht klar wird, welche Werte hier nun gemeint sind. Diese Schärfung des Verständnisses von beispielsweise „wichtigen Werten“, aber 
auch der Akteurinnen und Akteure, soll im Rahmen der vertiefenden Interviews stattfinden. In der Tabelle sind diejenigen Akteursebenen unterstrichen, denen die Aspekte zunächst zugeordnet werden sollen.

Betrachtet man Cronbachs Alpha, zeigt sich, dass die Dimension der Kulturation einen Wert von 0,59 erreicht, die Interaktion 0,73 und die Platzierung 0,72 (vgl. Tabelle 16). Für die Dimension der Identifikation konnte kein Wert errechnet werden, da diese nur ein Item enthält. Somit erreichen alle Dimensionen einen nach Lienert und Raatz (1994) akzeptablen Wert von Cronbachs Alpha von über 0,5. Im weiteren Verlauf der Arbeit wird daher die Struktur der vier Dimensionen sowie die Zuordnung der Items zu den Dimensionen beibehalten.

Auch eine Betrachtung, getrennt nach Akteursebenen ergibt akzeptable Werte von Cronbachs Alpha. Hier liegen die Werte bei 0,66 für Anforderungen an Migrantinnen und Migranten sowie bei 0,68 für Opportunitäten. Für Anforderungen an die Gesellschaft konnte ebenfalls kein Wert berechnet werden, da dieser Kategorie nur ein Item zugeordnet wurde. Insgesamt zeigt sich für die 13 Items zu Aspekten von Integration ein Wert von 0,82.

Tabelle 16: Werte für Cronbachs Alpha nach unterschiedlichen Dimensionen und Akteursebenen im Gesamtdatensatz

\begin{tabular}{ll|} 
Dimension/Akteursebene & $\begin{array}{l}\text { Cronbachs } \\
\text { Alpha }\end{array}$ \\
\hline Kulturation & 0,59 \\
\hline Interaktion & 0,73 \\
\hline Platzierung & 0,72 \\
\hline Identifikation & $/$ \\
\hline $\begin{array}{l}\text { Anforderung an Migrantinnen und } \\
\text { Migranten }\end{array}$ & 0,66 \\
\hline Opportunitäten & 0,68 \\
\hline Anforderungen an die Gesellschaft & $/$ \\
\hline Quelle: Eigene Darstellung. &
\end{tabular}

Quelle: Eigene Darstellung.

\subsubsection{Einstellungen zu verschiedenen Ausgängen von Integration}

Neben den Aspekten von Integration werden in dieser Arbeit auch Ausgänge von Integration betrachtet. Dazu fand zunächst eine Diskussion der Konzepte Assimilation und Pluralismus statt, welche beide in die Analyse eingehen sollen. Assimilation wird dabei als Anpassung verstanden und Pluralismus als ein Nebeneinander in der Gesellschaft, dass die Beibehaltung der eigenen Kultur ermöglicht. Zusätzlich wurden die vier möglichen Ausgänge nach Esser (2001) herangezogen, welche Segmentation, Marginalisierung, Assimilation und 
Mehrfachintegration umfassen. Zusätzlich zu den bereits festgelegten Ausgängen, die die Konzepte der Assimilation und des Pluralismus fokussieren, ermöglichen die Ausgänge nach Esser eine Erweiterung um die Ausgänge der Segmentation und Mehrfachintegration. Die Mehrfachintegration wird dabei auch als eine Ausgestaltung von pluralistischen Ansichten gesehen, die jedoch nicht auf die vollständige Beibehaltung der Kultur des Herkunftslandes ausgerichtet ist. Der Ausgang der Marginalisierung wird aus der Analyse ausgeschlossen, da die Abfrage wünschenswerte Ausgänge von Integration ermitteln soll. Die Marginalisierung stellt im Gegensatz dazu nämlich einen Zustand dar, in dem die Akteurin oder der Akteur in keines der gesellschaftlichen Systeme, also weder in die Herkunfts- noch in die Aufnahmegesellschaft, integriert ist.

Tabelle 17 zeigt die verwendeten Items und die Ausgänge von Integration, auf welche diese sich jeweils beziehen. Die Items beziehen sich auf kulturelle Bereiche, da die Assimilation in Bezug auf strukturelle Dimensionen/Aspekte als unstrittig angesehen wird, während eine Diskussion darüber besteht, inwiefern Assimilation auch im kulturellen Bereich nötig ist.

Die Abfrage der Einstellung zu unterschiedlichen Integrationskonzepten erfolgte anhand einer Skala von 1 „Stimme überhaupt nicht zu“ bis 5 „Stimme voll und ganz zu“. Dabei handelt es sich um eine Ordinalskala, die aber als quasimetrisch anzusehen ist, da, wie in den Sozialwissenschaften üblich, anzunehmen ist, dass die Abstände zwischen den Kategorien durch die Befragten gleich eingeschätzt werden (Völk1/Korb 2018: 20).

Tabelle 17: Verwendete Items zur Messung von Einstellungen zu unterschiedlichen Ausgängen von Integration

\begin{tabular}{l|ll}
$\begin{array}{l}\text { Ausgang von } \\
\text { Integration }\end{array}$ & Item & $\begin{array}{l}\text { Formulierung bei van Dick et al. } \\
\mathbf{( 1 9 9 7 )}\end{array}$ \\
\hline Segmentation & $\begin{array}{l}\text { Wenn Zuwanderer ihre Kultur } \\
\text { beibehalten möchten, sollten sie } \\
\text { unter sich bleiben. }\end{array}$ & $\begin{array}{l}\text { Wenn Mitglieder anderer ethnischer } \\
\text { Gruppen ihre Kultur beibehalten } \\
\text { möchten, sollten sie unter sich bleiben. }\end{array}$ \\
\hline Pluralismus & $\begin{array}{l}\text { Es wäre gut, wenn alle Zuwanderer } \\
\text { in Deutschland ihre Kulturen } \\
\text { beibehielten. }\end{array}$ & $\begin{array}{l}\text { Es wäre gut, wenn alle ethnischen } \\
\text { Gruppen in Deutschland ihre Kulturen } \\
\text { beibehielten. }\end{array}$ \\
\hline Assimilation & $\begin{array}{l}\text { Menschen, die nach Deutschland } \\
\text { kommen, sollten ihr Verhalten der } \\
\text { deutschen Kultur anpassen. }\end{array}$ & $\begin{array}{l}\text { Menschen, die nach Deutschland } \\
\text { kommen, sollten ihr Verhalten der } \\
\text { deutschen Kultur anpassen. }\end{array}$ \\
\hline Mehrfachintegration & $\begin{array}{l}\text { Zuwanderer sollten sowohl in ihre } \\
\text { Herkunftsgesellschaft als auch in } \\
\text { die deutsche Gesellschaft } \\
\text { eingebunden sein. }\end{array}$ & $/$ \\
\hline
\end{tabular}

Quelle: Eigene Darstellung. 
Die Items beziehen sich auf die Ausgänge Segmentation, Pluralismus, Assimilation und Mehrfachintegration. Dabei kommen die Ausgänge Segmentation, Assimilation und Mehrfachintegration im beschriebenen Modell von Esser (2001) vor. ${ }^{20}$ Zusätzlich wurde ein Item aufgenommen, das pluralistische Ansichten widerspiegeln soll, da in der vorgenommenen Diskussion um Assimilation und Pluralismus festgestellt wurde, dass gerade diesen beiden Ausgängen von Integration große Beachtung zukommt.

Wie in der Tabelle 17 abgebildet, sind drei Items an Formulierungen aus einer Arbeit von van Dick et al. (1997) angelehnt. Im Rahmen dieser Arbeit wurde ein Fragebogen zur Messung der Einstellung zur Akkulturation konstruiert. Hierbei nutzen die Verfassenden das Akkulturationsmodell nach Berry et al. (1989) und verstehen Akkulturation als „Veränderung der ursprünglich eingebrachten Kulturmuster von Gruppen mit unterschiedlichem kulturellem Hintergrund infolge fortgesetzten direkten Kontakts“ (van Dick et al. 1997: S. 2). Die Formulierungen wurden für die vorliegende Arbeit teilweise angepasst. So wurde der verwendete Begriff der ethnischen Gruppe durch den Begriff Zuwanderer ersetzt. Für den Ausgang der Mehrfachintegration wurde hingegen eine eigene Formulierung gewählt.

Darüber hinaus gehen weitere Variablen in die Analysen ein. Diese umfassen das Alter, die Zusammensetzung der Sportgruppe, den Bildungsstand, sowie den Migrationshintergrund.

\subsubsection{Sozialstrukturelle Merkmale der Befragten und Merkmale des Sportvereins}

In dieser Arbeit werden im Rahmen der sozialstrukturellen Merkmale der Befragten Alter, Bildungsstand und Migrationshintergrund einbezogen. In Bezug auf die Sportvereine wird die Zusammensetzung der Sportgruppe betrachtet.

Das Alter der Befragten liegt in Form von metrischen Variablen vor. Dazu wurde das Alter anhand einer Abfrage des Geburtsjahres erfasst.

Der Bildungsstand wird anhand einer ordinalen Variablen abgebildet. Der Bildungsstand der Befragten wurde anhand einer achtstufigen Ordinalskala der möglichen Bildungsabschlüsse abgefragt. Diese Abschlüsse umfassten:

- Noch Schüler

- Schule beendet ohne Abschluss

- Hauptschulabschluss (Volksschulabschluss)

\footnotetext{
${ }^{20}$ Siehe Kapitel 4.1.2 „Mehrfachintegration, Assimilation, Marginalität und Segmentation nach Hartmut Esser“
} 
- Realschulabschluss (Mittlere Reife)

- Polytechnische Oberschule 10. Klasse

- Fachhochschulreife (Abschluss einer Fachoberschule)

- Abitur (Hochschulreife)

- Andere

Ausgeschlossen wurden in den späteren Analysen Schülerinnen und Schüler, die noch keinen Abschluss erlangt haben oder die Schule ohne Abschluss beendet haben, sowie Personen mit anderen Abschlüssen, da diese Gruppen sehr gering besetzt waren. Personen mit einem Abschluss an einer polytechnischen Oberschule wurden dem Realschulabschluss zugeordnet.

In Bezug auf den Migrationshintergrund liegt hingegen eine dichotome Variable vor. Um Personen mit Migrationshintergrund im Datensatz zu identifizieren, wurde die Frage danach, ob die Person in Deutschland geboren wurde, sowie die Frage nach dem Geburtsland der Eltern herangezogen. Zu den Personen mit Migrationshintergrund zählen demnach alle Personen, die selbst nicht in Deutschland geboren wurden oder mindestens ein Elternteil besitzen, das im Ausland geboren wurde. Bei späteren Analysen wird außerdem zwischen Migrantinnen und Migranten erster sowie zweiter Generation unterschieden. Als Migrantinnen und Migranten erster Generation gelten dabei Personen mit eigener Migrationserfahrung. Unter Migrantinnen und Migranten der zweiten Generation werden Personen gefasst, die mindestens ein Elternteil haben, das im Ausland geboren wurde und selbst keine eigene Migrationserfahrung besitzen. Eine weitere Analyse bezieht sich außerdem auf die Herkunft von Personen bzw. deren Eltern. Dazu wurde anhand des Geburtslandes oder der Geburtsländer der Eltern eine Zuordnung zu einem europäischen oder außereuropäischen Migrationshintergrund vorgenommen. Wurde ein Elternteil in einem außereuropäischen und das andere Elternteil in einem europäischen Land geboren, wurde ein fehlender Wert hinterlegt.

Für die Zusammensetzung der Sportgruppe wurden die Befragten gebeten, den Anteil von Personen mit Migrationshintergrund in ihrer Sportgruppe zu schätzen. Es handelt sich somit um eine metrische Variable. $\mathrm{Zu}$ beachten ist hierbei, dass die Angaben subjektive Einschätzungen der Befragten darstellen, die von der realen Zusammensetzung der Sportgruppe abweichen können. 


\subsubsection{Quantitative Auswertungsmethoden}

Wie bereits dargestellt, gliedert sich die Analyse dieser Arbeit in ein exploratives und hypothesengeleitetes Vorgehen. Insgesamt sind dabei folgende Fragestellungen von Relevanz:

- Welche subjektiven Sichtweisen von Integration (in Form von Verständnis von Integration und Einstellungen zu Integrationskonzepten/Ausgängen von Integration) haben die Akteurinnen und Akteure in Sportorganisationen?

- Welche Dimensionen und Aspekte von Integration sind ihnen dabei wichtig?

- Welche Ergebnisse von Integration werden für wünschenswert gehalten?

- Wie hängen Ergebnisse und Aspekte von Integration zusammen?

- Inwiefern variieren die Sichtweisen auf Integration in Abhängigkeit von sozialstrukturellen Faktoren und der Zusammensetzung der Mitglieder der Sportvereine?

Das explorative Vorgehen bezieht sich auf die ersten vier Fragestellungen, während zur Beantwortung der letzten Fragestellung, die in Kapitel 4.3 aufgestellten Hypothesen getestet werden.

In Bezug auf die Beantwortung der ersten vier Fragen zu Sichtweisen auf Integration sollen größtenteils deskriptive Analysen in Form von Häufigkeitsauszählungen und der Analyse von Mittelwerten durchgeführt sowie die Ergebnisse der unterschiedlichen Akteursebenen und die zwischen Personen mit und ohne Migrationshintergrund verglichen werden. Dabei soll auch überprüft werden, ob signifikante Unterschiede in Bezug auf die Bewertung von Aspekten und Ausgängen von Integration zwischen Personen mit und ohne Migrationshintergrund vorliegen. Hierzu werden t-Tests durchgeführt. Mit Hilfe des t-Tests kann ermittelt werden, ob zwei Gruppen in einem Merkmal einen Unterschied aufweisen. Dazu werden die Mittelwerte der beiden Gruppen in Bezug auf das untersuchte Merkmal herangezogen (Rasch et al. 2014a: 33). Zur Durchführung des t-Tests müssen insgesamt drei Voraussetzungen erfüllt sein. Diese umfassen das Intervallskalenniveau der untersuchten Variable, die Normalverteilung in der Population und die Varianzhomogenität der beiden untersuchten Gruppen in Bezug auf das untersuchte Merkmal. Der t-Test reagiert zwar robust gegenüber Verletzungen dieser Voraussetzungen, dies ist aber nicht der Fall, wenn die Gruppengrößen zu klein sind oder sich in ihrer Größe zu stark unterscheiden $(\mathrm{n} 1=\mathrm{n} 2>30)$ (Rasch et al. 2014a: 43). Dies ist hier bei beiden Gruppenvergleichen der Fall, da sich die Fallzahl von 422 Befragten in 344 Befragte ohne Migrationshintergrund und 78 mit Migrationshintergrund sowie 85 befragte Vorstände 
und 337 Mitglieder gliedern. Darüber hinaus weisen die Variablen zur Bewertung der Aspekte und Ziele von Integration keine Normalverteilungen auf ${ }^{21}$ und die Voraussetzung der Varianzhomogenität ist beim Vergleich der Gruppen Personen mit und ohne Migrationshintergrund sowie Vorstände und Mitglieder bei einzelnen Variablen nicht gegeben $^{22}$. Aus diesem Grund wird zusätzlich ein Mann-Whitney U-Test durchgeführt, welcher bei Variablen vorgezogen wird, bei denen die Annahme der Varianzhomogenität verletzt ist. Beim U-Test handelt es sich um ein nicht-parametrisches Verfahren, mit dem Unterschiede zwischen den Gruppen anhand von Rangplätzen errechnet werden (Rasch et al. 2014b: 94).

Die Zusammenhänge zwischen Aspekten und Ergebnissen von Integration könnten aufgrund des Skalenniveaus mit Hilfe des Pearson-Korrelationskoeffizienten analysiert werden. Da der Pearson-Korrelationskoeffizient für die Interpretation der Signifikanz jedoch eine Normalverteilung der Variablen voraussetzt, welche in der Stichprobe nicht gegeben ist, werden die Zusammenhänge mit Hilfe von Spearman's rho analysiert.

Im Rahmen des hypothesengeleiteten Teils der Arbeit werden die in Kapitel 4.3 aufgestellten Zusammenhangshypothesen untersucht. Aufgrund der unterschiedlichen Skalenniveaus der verwendeten Variablen sollten für die Ermittlung der Zusammenhänge unterschiedliche Zusammenhangsmaße verwendet werden. Die Zusammenhänge mit dem Alter, dem Bildungsstand und der Zusammensetzung der Sportgruppe werden mit Hilfe von Spearman's rho analysiert. Der Zusammenhang mit der dichotomen Variable zum Migrationshintergrund wird anhand von Eta untersucht.

Insgesamt handelt es sich bei allen durchgeführten Analysen zu Zusammenhängen um bivariate Analysen. Diese Verfahren wurden aufgrund der geringen Fallzahlen gewählt.

\subsection{Qualitative Methoden}

Dieses Unterkapitel beschreibt die verwendeten qualitativen Methoden sowie deren Analyseverfahren. Innerhalb der qualitativen Untersuchung, die ergänzend zu den quantitativen Methoden herangezogen werden, wurden sowohl explorative als auch vertiefende LeitfadenInterviews $^{23}$ geführt.

\footnotetext{
${ }^{21}$ Siehe Anhang

${ }^{22}$ Siehe Anhang
} 


\subsubsection{Explorative Interviews mit Vereinsvorständen}

Im Rahmen des Projekts „InBewegung“ dienten die explorativen Interviews als erste Annäherung an den Forschungsgegenstand sowie dazu, weitere Themen für die quantitative Befragung aufzuzeigen. Die Dauer der einzelnen Interviews betrug circa 45 bis 80 Minuten. Darüber hinaus wurden die Interviews mit Einverständnis der interviewten Personen aufgezeichnet und anschließend verschriftlicht sowie anonymisiert.

Insgesamt wurden Interviews mit acht Vereinsvorständen $\mathrm{zu}$ den Themenblöcken Vereinskultur, Integrationsprojekte des Vereins, Veränderungsprozesse und Bedarfe und Verständnis von Integration/Umgang mit Vielfalt geführt. Zusätzlich zu den Themenblöcken wurde ein Leitfaden konstruiert ${ }^{24}$, der die Themenblöcke in einzelne mögliche Fragestellungen differenziert. Dieser Leitfaden diente dazu, das Gespräch zu strukturieren und darüber hinaus eine gewisse Vergleichbarkeit über die verschiedenen Interviews hinweg zu gewährleisten. Jedoch stellt dieser Leitfaden nur eine Vorlage in Form eines Gerüsts dar und war nicht zwingend in seinem Ablauf wie vorgegeben umzusetzen, da die Interviews ihren explorativen Charakter nicht verlieren sollten. Es handelt sich somit um teilstrukturierte Interviews. Dieses Vorgehen erschien geeignet, da mit Hilfe der explorativen Interviews ein möglichst umfassendes Bild der Arbeit in den Vereinen abgebildet werden sollte.

Für diese Arbeit dienen die explorativen Interviews der Vertiefung der quantitativen Ergebnisse, da im Rahmen der Interviews nicht nur die Bedeutung unterschiedlicher Dimensionen und Aspekte von Integration sowie die Einstellung $\mathrm{zu}$ bestimmten Integrationskonzepten erläutert werden kann, sondern auch erwartete Wirkweisen zwischen unterschiedlichen Aspekten oder Dimensionen von Integration. Von Bedeutung ist dabei der Themenblock zu Verständnis von Integration bzw. Umgang mit Vielfalt. Im Rahmen dieses Themenblocks wurde den Befragten die Frage „Was bedeutet für Sie persönlich Integration?“ gestellt. Jedoch zeigte sich in der direkten Abfrage, dass viele Befragte auf diese Frage keine Antwort fanden oder wenn, nur kurze Ausführungen machten. Neben der expliziten Frage nach dem eigenen Verständnis von Integration, dienen darüber hinaus deshalb vor allem Ausführungen zu Integrationsprojekten und Veränderungsprozessen und Bedarfen der Analyse, da auch in diesen Themenblöcken Vorstellungen von Integration sichtbar wurden, bzw. in den Ausführungen mitgeschwungen sind.

\footnotetext{
${ }^{24}$ Siehe Anhang
} 


\subsubsection{Vertiefende Interviews mit Vereinsmitgliedern}

Die auf die explorativen Interviews folgenden Interviews mit Vereinsmitgliedern dienen der weiteren Vertiefung einzelner Aspekte und Dimensionen von Integration, die im Rahmen der explorativen Interviews nicht in detaillierterer Form ausgeführt wurden. Darüber hinaus, sollen aber auch die Sichtweisen der Mitglieder im Vergleich zu den Vereinsvorständen abgebildet werden. Insgesamt wurden fünf Interviews geführt und zwei der Personen besitzen eine eigene Migrationserfahrung. Die Dauer der einzelnen Interviews betrug circa 15 bis 25 Minuten. Darüber hinaus wurden die Interviews mit dem Einverständnis der jeweiligen interviewten Person aufgezeichnet und anschließend transkribiert sowie anonymisiert.

Im Gegensatz zu den explorativen Interviews, bei denen es sich um teilstrukturierte und an Themenblöcken orientierte Interviews handelte, wurden die vertiefenden Interviews mit Hilfe eines verbindlichen Leitfadens durchgeführt. Dieser Leitfaden ist in Tabelle 18 dargestellt.

Tabelle 18: Leitfaden für die vertiefenden Interviews

\begin{tabular}{|c|c|}
\hline Block & Fragen \\
\hline Einstieg & $\begin{array}{l}\text { - Was sind Ihre ersten Gedanken, wenn Sie den Begriff } \\
\text { „Integration“ hören? } \\
\text { - An welche Personengruppen denken Sie, wenn Sie den Begriff } \\
\text { „Integration“ hören? }\end{array}$ \\
\hline $\begin{array}{l}\text { Ausgestaltung von } \\
\text { Integration }\end{array}$ & $\begin{array}{l}\text { - Was sollten diese Personen tun, um integriert zu sein? } \\
\text { - Sind für Integration auch Beiträge von anderen Seiten nötig? } \\
\text { Wenn ja, welche weiteren Beiträge sind nötig und von wem } \\
\text { müssen diese erfüllt werden? } \\
\text { - Wer ist verantwortlich dafür, dass Integration funktioniert? } \\
\text { - Wenn es einen Integrationsbarometer gäbe, was würde dieser } \\
\text { - Wessen? }\end{array}$ \\
\hline $\begin{array}{l}\text { Vorstellungen zu } \\
\text { einzelnen } \\
\text { Aspekten und } \\
\text { Dimensionen }\end{array}$ & $\begin{array}{l}\text { In Zusammenhang mit Integration wird immer wieder gesagt, } \\
\text { dass wichtige Werte anerkannt werden sollten. Was glauben Sie, } \\
\text { welche Werte das sind? } \\
\text { - Welche Rolle spielt der Bildungsstand oder die berufliche } \\
\text { Stellung für Integration? } \\
\text { Wie wichtig ist ein vorhandenes Zugehörigkeitsgefühl für } \\
\text { Integration? Wie erhält man ein Gefühl von Zugehörigkeit? }\end{array}$ \\
\hline $\begin{array}{l}\text { Ziele von } \\
\text { Integration und } \\
\text { Abschluss }\end{array}$ & $\begin{array}{l}\text { - Was ist nach Ihrer Meinung das Ziel von Integration? Wie sollte } \\
\text { eine Gesellschaft aussehen, in der alle integriert sind? }\end{array}$ \\
\hline
\end{tabular}


- Was würden Sie abschließend sagen: Was bedeutet für Sie persönlich Integration?

Quelle: Eigene Darstellung.

\subsubsection{Qualitative Auswertungsmethoden}

Für die Analyse der explorativen sowie der vertiefenden Interviews wird die Methode der qualitativen Inhaltsanalyse nach Mayring (2008) genutzt, da anhand des theoretischen Rahmens mit einem deduktiven Kategoriensystem gearbeitet werden soll. Die Analyse erfolgt mit dem Programm MAXQDA. Um eine Vergleichbarkeit der Analysen der explorativen und vertiefenden Interviews zu gewährleisten, wird bei beiden Interviewformen das gleiche Kodierschema verwendet.

Die Inhaltsanalyse ist ein systematischer Ansatz, der sich an Regeln orientiert (Mayring 2008: 42). Aus diesem Grund muss das Analyseinstrument an das Forschungsobjekt, die Forschungsfrage und auch an das Analysematerial angepasst werden. Dies geschieht im Rahmen der Entwicklung eines Kategoriensystems (Mayring 2008: 42). Zur Entwicklung des Kategoriensystems kann ein induktives oder deduktives Vorgehen gewählt werden. Der induktive Ansatz umfasst eine Ableitung der Kategorien aus dem Datenmaterial, während der deduktive Ansatz mit zuvor entworfenen Kategorien und mit Ankerbeispielen sowie Kodierregeln arbeitet. Die Kategorien werden dazu aus dem verwendeten theoretischen Rahmen abgeleitet (Vgl. Mayring 2000).

Im Rahmen dieser Arbeit wird mit einem deduktiven Kategorienschema gearbeitet, welches aus dem zuvor vorgestellten theoretischen Rahmen abgeleitet wurde. Die Kategorien, Kodierregeln und Ankerbeispiele sind in Tabelle 19 dargestellt.

Tabelle 19: Kategorien, Beschreibungen und Ankerbeispiele für die qualitative Inhaltsanalyse

\begin{tabular}{ll} 
Kategorie & Beschreibung/Ankerbeispiel \\
\hline Kulturation & $\begin{array}{l}\text { Äußerungen, die sich auf die Aneignung von Wissen und } \\
\text { Kompetenzen, die für ein erfolgreiches Agieren und Interagieren nötig } \\
\text { sind, beziehen sowie Fähigkeiten für die Bewältigung typischer } \\
\text { Situationen (bspw. Sprache). }\end{array}$ \\
& $\begin{array}{l}\text { Beispiel: "Also ich arbeite gerade an den Regeln und die Regeln in der } \\
\text { letzten Saison waren, dass auf dem Sportplatz und auf dem } \\
\text { Sportgelände nur Deutsch gesprochen wird und ich erweitere das jetzt, } \\
\text { dass bei jeder Aktivität, bei der wir sind, Deutsch gesprochen wird“ } \\
\text { (I4: 170-173). }\end{array}$ \\
\hline $\begin{array}{l}\text { Äußerungen, die sich auf ,die Besetzung einer bestimmen } \\
\text { gesellschaftlichen Position durch einen Akteur“ (Esser 2001: 9) }\end{array}$ \\
\hline Platzierung
\end{tabular}


beziehen sowie Fertigkeiten und Ressourcen (bspw. Arbeitskraft), die durch andere Akteure nachgefragt werden können.

Beispiel: “...macht jetzt eine Ausbildung als Bauzeichner, wird wahrscheinlich auch noch studieren ja, lebt mit einer deutschen Freundin zusammen, haben eine eigene Wohnung, ..." (I4: 413-415).

\begin{tabular}{l|l}
\hline Identifikation & $\begin{array}{l}\text { Äußerungen, die sich auf kollektive Orientierung beziehen. } \\
\text { Beispiel: „Und die Tendenz ist ja eher tatsächlich so, dass jeder } \\
\text { individuell für sich sucht und wir haben gesagt, jeder der bei uns } \\
\text { Mitglied werden muss, der muss auch diese Leitlinien unterschreiben } \\
\text { (I6: 25-27).“ }\end{array}$ \\
\hline Interaktion & $\begin{array}{l}\text { Äußerungen, die sich auf Aspekte der Interaktion beziehen. Dazu } \\
\text { gehören beispielsweise Möglichkeiten des Zusammentreffens, } \\
\text { Kontakte, Freundschaften, Beziehungen, Begegnungen. }\end{array}$
\end{tabular}

Beispiel: „Also sich einfach auch gut miteinbringen, das ist für mich gelungene Integration, wenn einer von sich aus, auch wenn viel von uns kommt, aber auch selbst bereit ist von sich aus anzupacken und zu steuern und schnell auch Freundschaften, auch außerhalb seines afghanischen Kleinkreises findet, das finde ich Integration, ja“ (I4: 440-444).

\begin{tabular}{|c|c|}
\hline & $\begin{array}{l}\text { Personengruppen oder Personen, auf die mit dem Begriff Integration } \\
\text { Bezug genommen wird sowie Personengruppen oder Personen, die in } \\
\text { Bezug auf Integration zum Handeln angehalten werden. } \\
\text { Beispiel: „Also das, dieser ganze Integrationsprozess, erfordert eben } \\
\text { sehr viel Einfühlungsvermögen und das wird nicht, dazu braucht man } \\
\text { viele Informationen, ja. Um zu verstehen, was die jungen Menschen } \\
\text { auch, die hierhergekommen sind, was die bewogen hat } \\
\text { hierherzukommen und so weiter“ (I2: 203-206). }\end{array}$ \\
\hline & $\begin{array}{l}\text { Äußerungen, die auf Ergebnisse von Integration und vor allem auf } \\
\text { pluralistische und assimilative Tendenzen anspielen. } \\
\text { Beispiel: ,... dass es keine und man merkt gar nicht mehr, dass er von } \\
\text { irgendwo anders her, also er ist, außer dass man es sieht von seiner } \\
\text { Hautfarbe ja von seinem Äußeren, hat er aber, er kann perfekt Deutsch, } \\
\text { er ist schon so weit, dass er so Witze versteht ja so doppeldeutige } \\
\text { Witze und so weiter“ (I4:431-434). }\end{array}$ \\
\hline $\begin{array}{l}\text { Gegenüberstellung } \\
\text { mit anderen } \\
\text { Konzepten }\end{array}$ & $\begin{array}{l}\text { Abgrenzungen } \mathrm{zu} \text { anderen Konzepten sowie Darstellung von } \\
\text { Zusammenhängen bzw. Übereinstimmungen. } \\
\text { Beispiel: „Wir sehen da eine Gemeinsamkeit, deshalb haben wir beide } \\
\text { Felder zusammengenommen, Kultur und Integration, weil das eine } \\
\text { auch mit dem anderen zusammenhängt und ja, ich bin zuständig für } \\
\text { diesen Bereich“(I1: 5-7). }\end{array}$ \\
\hline
\end{tabular}

Quelle: Eigene Darstellung. 
Die Gegenüberstellung der theoretischen Konzepte hatte zunächst gezeigt, dass in den Konzeptionen überwiegend auf vier Dimensionen von Integration abgestellt wird. Aus diesem Grund bilden diese vier Dimensionen, orientiert an den Bezeichnungen von Esser (2001), die ersten vier Kategorien des Schemas. Dabei werden alle Aspekte kodiert, die sich innerhalb der Konzeptionen unter die einzelnen Dimensionen fassen lassen.

Darüber hinaus hatte sich bei der Betrachtung der unterschiedlichen Konzeptionen auch gezeigt, dass mit der Zeit von der Vorstellung, Integration wäre nur auf Leistung der Migrantinnen und Migranten begründet, Abstand genommen wurde und weitere Adressatinnen und Adressaten in Form von Opportunitäten in der Gesellschaft, Leistungen der aufnehmenden Gesellschaft sowie emotionale Aspekte von Bedeutung sein können. Äußerungen in Bezug auf unterschiedliche Adressatinnen und Adressaten werden mit der Kategorie „Adressatinnen und Adressaten von Integration“ kodiert. Außerdem fallen unter diese Kategorie Personen und Personengruppen, die mit dem Begriff Integration in Verbindung gebracht werden und somit als ,zu integrierende Gruppe“ dargestellt werden.

Die Kategorie „Ergebnisse von Integration“ bezieht sich auf Ausgänge von Integration, die von den befragten Personen beschrieben oder als wünschenswert dargestellt werden. Dabei soll vor allem zwischen assimilativen und pluralistischen Tendenzen unterschieden werden. Die letzte Kategorie „Gegenüberstellung mit anderen Konzepten“ soll dazu dienen, Differenzierungen, die die befragten Personen vornehmen, darzustellen.

Die qualitative Inhaltsanalyse eignet sich für die explorativen Fragen dieser Arbeit insbesondere, da damit die Aussagen der Befragten systematisiert und auch einzelne Kategorien gegenübergestellt werden können. Darüber hinaus können Kategorien aus dem theoretischen Rahmen abgeleitet werden. Es handelt sich somit um ein theoriegeleitetes Vorgehen und die Analyse erfolgt auf der Ebene des Gesagten. Dies steht im Gegensatz zu Analyseverfahren wie beispielsweise der Grounded Theory, bei der ein theoriegenerierendes Vorgehen vorliegt und die Kodierung wesentlich offener erfolgt (Glaser/Strauss 2010).

Das theoriegeleitete Vorgehen mit zuvor abgeleitetem Kategorienschema bietet sich auch an, da die qualitativen Daten aus den Interviews ergänzend und vertiefend zu den quantitativen Daten herangezogen werden sollen. So können Ergebnisse in einzelnen Kategorien der quantitativen Befragung mit den Ergebnissen der jeweiligen Kategorie aus den Interviews ergänzt werden. 


\subsection{Integration der Daten}

Im Rahmen der vorliegenden Arbeit besteht die Aufgabe der Methodenkombination in der Vervollständigung der quantitativen Daten (Komplementarität), um die Ergebnisse der quantitativen Erhebung weiter zu hinterfragen und auszuführen. Dazu soll im quantitativen Teil der Arbeit vor allem die Bedeutung einzelner Dimensionen und Aspekte von Integration, die Unterstützung unterschiedlicher Ausgänge von Integration sowie Zusammenhänge zwischen der Unterstützung unterschiedlicher Ausgänge von Integration und sozialstrukturellen Merkmalen sowie Merkmalen der Sportvereine untersucht werden. Im Rahmen der qualitativen Auswertungen sollen einzelne Aspekte weiter ausgeführt sowie die erwarteten Wirkweisen zwischen Aspekten und Dimensionen im Integrationsprozess abgebildet werden. Eine Integration der unterschiedlichen Datentypen ist somit für die Beantwortung der Forschungsfrage unerlässlich. Es handelt sich um ein sequenzielles vertiefendes Design, bei dem die quantitative Erhebung gegenüber der qualitativen Untersuchungen Priorität erhält. Die Zusammenführung der Daten erfolgt im Rahmen der Datenanalyse. Den theoretischen Rahmen bilden die Ausführungen zu Dimensionen und Aspekten sowie Ergebnissen von Integration, mit deren Hilfe zunächst ein fundiertes Set an Items für die quantitative Befragung erstellt wurde. 


\section{Ergebnisse}

In diesem Kapitel werden die Ergebnisse der quantitativen und qualitativen Analysen dargestellt. Dazu werden im nächsten Unterkapitel zunächst die Ergebnisse der quantitativen Analyse beschrieben. Darauf folgen die Ergebnisse der explorativen Interviews mit Vereinsvorständen sowie der vertiefenden Interviews mit Vereinsmitgliedern. Anschließend werden die quantitativen und qualitativen Ergebnisse in einem weiteren Kapitel zusammengeführt sowie in einem abschließenden Kapitel diskutiert.

\subsection{Quantitative Befragung von Vereinsvorständen und Mitgliedern}

Im Rahmen dieses Kapitels werden die Ergebnisse der quantitativen Analysen dargestellt. Zunächst folgen deskriptive Analysen der Aspekte und Ausgänge von Integration, die auch die Analyse der Gruppenvergleiche zwischen Vereinsvorständen und Vereinsmitgliedern sowie Personen mit und ohne Migrationshintergrund beinhalten. Darauf folgen Darstellungen zu Zusammenhängen zwischen Ergebnissen und Aspekten von Integration sowie zwischen der Einstellung zu Integrationskonzepten, sozialstrukturellen Merkmalen und Merkmalen der Sportvereine. Während die ersten deskriptiven Analysen sowie die Darstellungen zu Zusammenhängen zwischen Ergebnissen und Aspekten von Integration dem explorativen Vorgehen dieser Arbeit zuzurechnen sind, werden im Rahmen der Prüfung der Zusammenhänge zwischen Ergebnissen von Integration und sozialstrukturellen Merkmalen bzw. Merkmalen der Sportvereine die aufgeworfenen Hypothesen überprüft. Den Abschluss dieses Unterkapitels bilden die Zusammenfassung und Diskussion der Ergebnisse.

\subsubsection{Deskriptive Analyse}

Im Rahmen der deskriptiven Analyse erfolgt zunächst die deskriptive Beschreibung der Items anhand des Gesamtdatensatzes. Im Rahmen dieser Analyse spielt vor allem die Bedeutung, die den einzelnen Aspekten und Ausgängen von Integration beigemessen wird, eine Rolle. Im Rahmen der Analyse soll herausgearbeitet werden, welche Aspekte als besonders bedeutsam für Integration eingeschätzt werden und welche Ausgänge von Integration die höchste Zustimmung erfahren. Dabei werden Häufigkeitsverteilungen, Mittelwerte sowie Vergleiche zwischen Vereinsvorständen und Vereinsmitgliedern sowie Personen mit und ohne Migrationshintergrund dargestellt. 


\subsubsection{Bewertung der Aspekte von Integration}

Im Folgenden werden die Verteilungen der einzelnen Items für Aspekte und Ausgänge von Integration beschrieben. Im Anschluss daran werden die Mittelwerte der Items verglichen, um herauszustellen, welche Aspekte von den Befragten als besonders bedeutsam für Integration gesehen werden und welche Integrationskonzepte die höchste Zustimmung erfahren.

Betrachtet man zunächst die ersten beiden im Fragebogen genannten Aspekte zu guten Deutschkenntnisse und der Anerkennung wichtiger Werte, welche beide der Dimension der Kulturation zuzuordnen sind und sich auf Anforderungen an die zu integrierenden Personen beziehen, zeigt sich, dass der Anerkennung wichtiger Werte mehr Bedeutung zugemessen wird als Deutschkenntnissen (vgl. Abbildung 5). Insgesamt bewerten 82,1\% der Befragten den Aspekt der Anerkennung wichtiger Werte als (eher) wichtig. In Bezug auf die Deutschkenntnisse sind es 68,4\%. Demnach kommt der Anerkennung wichtiger Werte innerhalb der Dimension der Kulturation die größte Bedeutung zu.

Abbildung 5: Verteilung der Items der Dimension Kulturation in Prozent

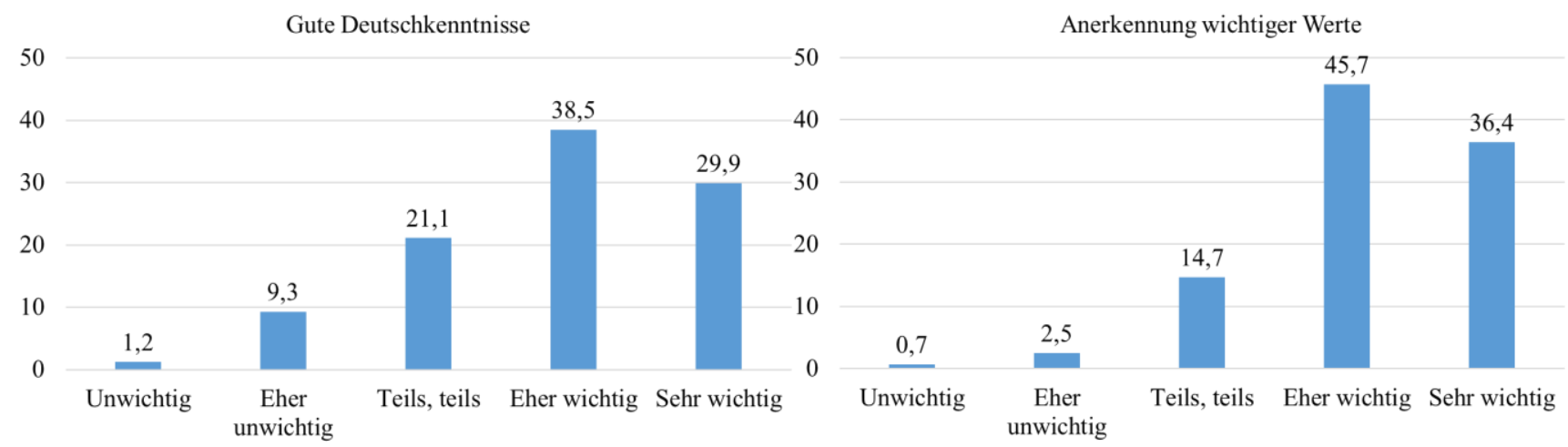

Quelle: Eigene Darstellung; Gute Deutschkenntnisse n =408, Anerkennung wichtiger Werte n $=407$.

Unter die Dimension der Interaktion wurden insgesamt fünf Items gefasst (vgl. Abbildung 6). Diese umfassen Chancen zur Teilnahme an der Gesellschaft, den Willen, an der Gesellschaft teilzunehmen, Kontakte zwischen Menschen unterschiedlicher Kulturen, Mitgliedschaft/Engagement in Organisationen und Anerkennung durch Andere. Betrachtet man die fünf Items, zeigt sich der höchste Zuspruch in Form der Bewertung als (eher) wichtig beim Willen an der Gesellschaft teilzunehmen (89,9\%). Jedoch werden auch Chancen zur Teilnahme an der Gesellschaft hoch bewertet. Diesen Aspekt bewerten 87,7 \% der Befragten als (eher) wichtig. Die Teilnahmechancen und -bereitschaft werden somit als bedeutender für Integration eingeschätzt als interethnische Kontakte (80,9 \%), Anerkennung durch Andere (65,7\%) und Mitgliedschaft/Engagement in Organisationen (33,4 \%). Insgesamt zeigen sich hier bei allen fünf Items nur geringe Anteile in der Einschätzung dieser Aspekte als (eher) unwichtig. Eine 
Ausnahme bildet das Item Mitgliedschaft/Engagement in Organisationen, welches von 16,8 \% der Befragten als (eher) unwichtig eingestuft wird. Gleichzeitig zeigt sich bei den Items Anerkennung durch Andere sowie Mitgliedschaft/Engagement in Organisationen eine deutlich höhere Besetzung der Mittelkategorie als bei den anderen Items. Beim Item Anerkennung durch andere gaben 26,2 \% der Befragten „Teils, teils“ an und bei Mitgliedschaft/Engagement in Organisationen sogar 39,9 \%. Dies könnte eine gewisse Unsicherheit bei der Bewertung, in Form des Ausweichens auf die Mittelkategorie, bezüglich dieser Items widerspiegeln.

Der Dimension der Identifikation wurde lediglich das Item „Vorhandensein eines Zugehörigkeitsgefühls“ zugeordnet (vgl. Grafik rechts unten in Abbildung 6). Hier geben die meisten Befragten „Eher wichtig“ (52,6 \%) an. Insgesamt liegt die Zustimmung in Bezug auf die Wichtigkeit bei diesem Item bei 85,9\%.

Abbildung 6: Verteilung der Items der Dimensionen Interaktion und Identifikation in Prozent

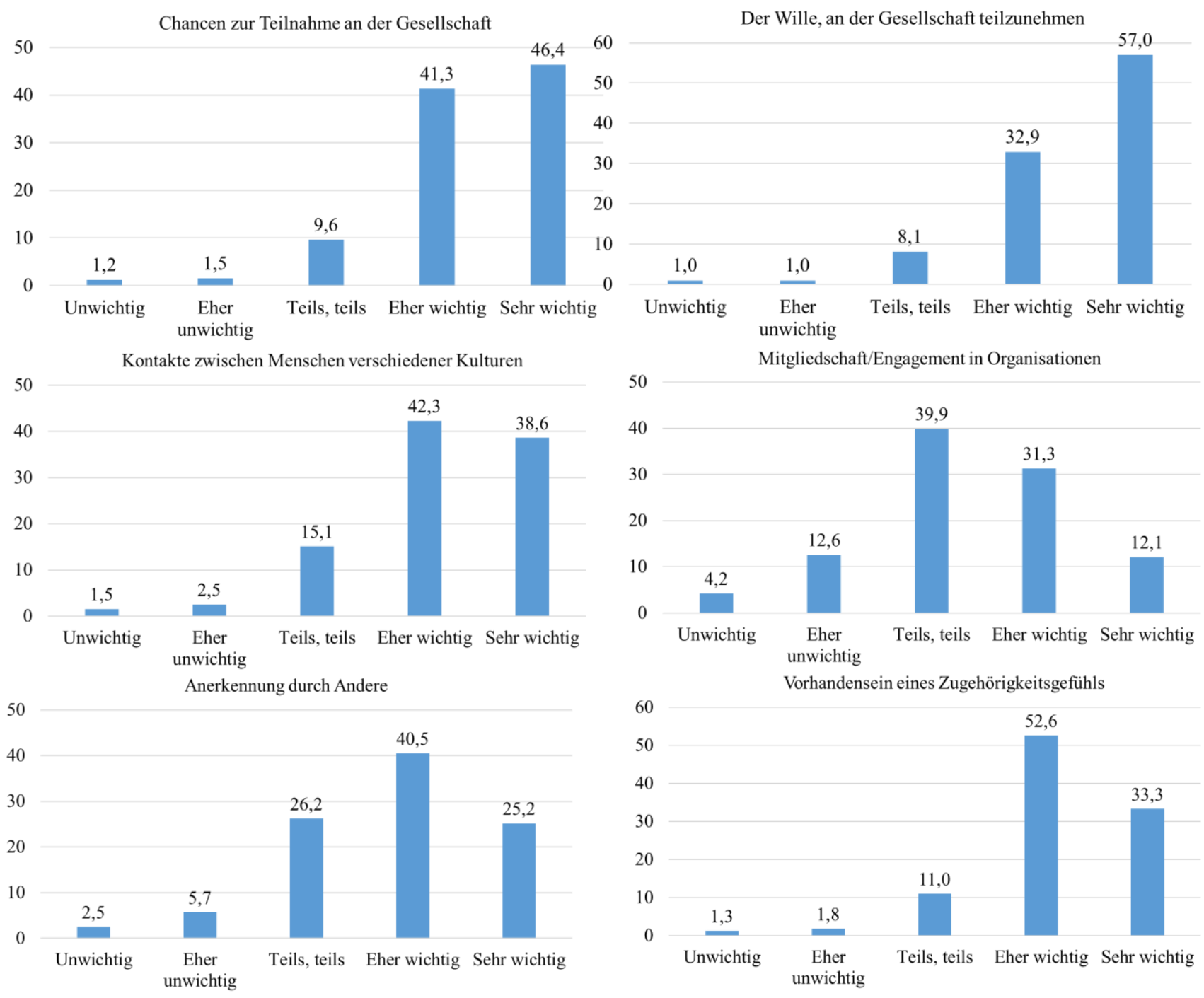

Quelle: Eigene Darstellung; Chancen zur Teilnahme an der Gesellschaft $n=407$, Der Wille an der Gesellschaft teilzunehmen $\mathrm{n}=407$, Kontakte zwischen Menschen verschiedener Kulturen $n=404$, Mitgliedschaft/Engagement in Organisationen $n=406$, Anerkennung durch Andere n = 405, Vorhandensein eines Zugehörigkeitsgefühls n = 399 . 
Die größten Differenzen zwischen Einzelitems zeigen sich bei der Dimension der Platzierung, welche die Items Gleiche Rechte für Alle, Bildungsstand, berufliche Stellung, Zugang zum Wohnungsmarkt und Zufriedenheit mit beruflicher und sozialer Situation enthält (vgl. Abbildung 7). Innerhalb dieser Dimension werden die Items Gleiche Rechte für Alle (94,8 \%) und Zufriedenheit mit der beruflichen und sozialen Stellung $(73,8 \%)$ mit der größten Zustimmung als (eher) wichtige Aspekte angesehen. Gleichzeitig stellt die Forderung nach gleichen Rechten für alle auch insgesamt den am höchsten bewerteten Aspekt innerhalb der Fragebatterie dar. Der Zugang zum Wohnungsmarkt wird mit 62,5\% ebenfalls als (eher) wichtig bewertet.

Das Gegenteil zeigt sich hingegen in Bezug auf den Bildungsstand und die berufliche Stellung. Den Bildungsstand sehen nur 30,0 \% als (eher) wichtigen Aspekt der Integration an. 35,6 \% der Befragten sehen den Bildungsstand sogar als (eher) unwichtig an und 34,4\% geben „Teils, teils“ an. Die berufliche Stellung sehen noch weniger der Befragten als (eher) wichtigen Aspekt an $(14,1 \%)$. Hier schätzen 53,0 \% der Befragten das Item als (eher) unwichtig ein. Der Bildungsstand und die berufliche Stellung stellen somit die beiden Aspekte dar, die als am wenigsten wichtig für Integration angesehen werden. 
Abbildung 7: Verteilung der Items der Dimension Platzierung in Prozent
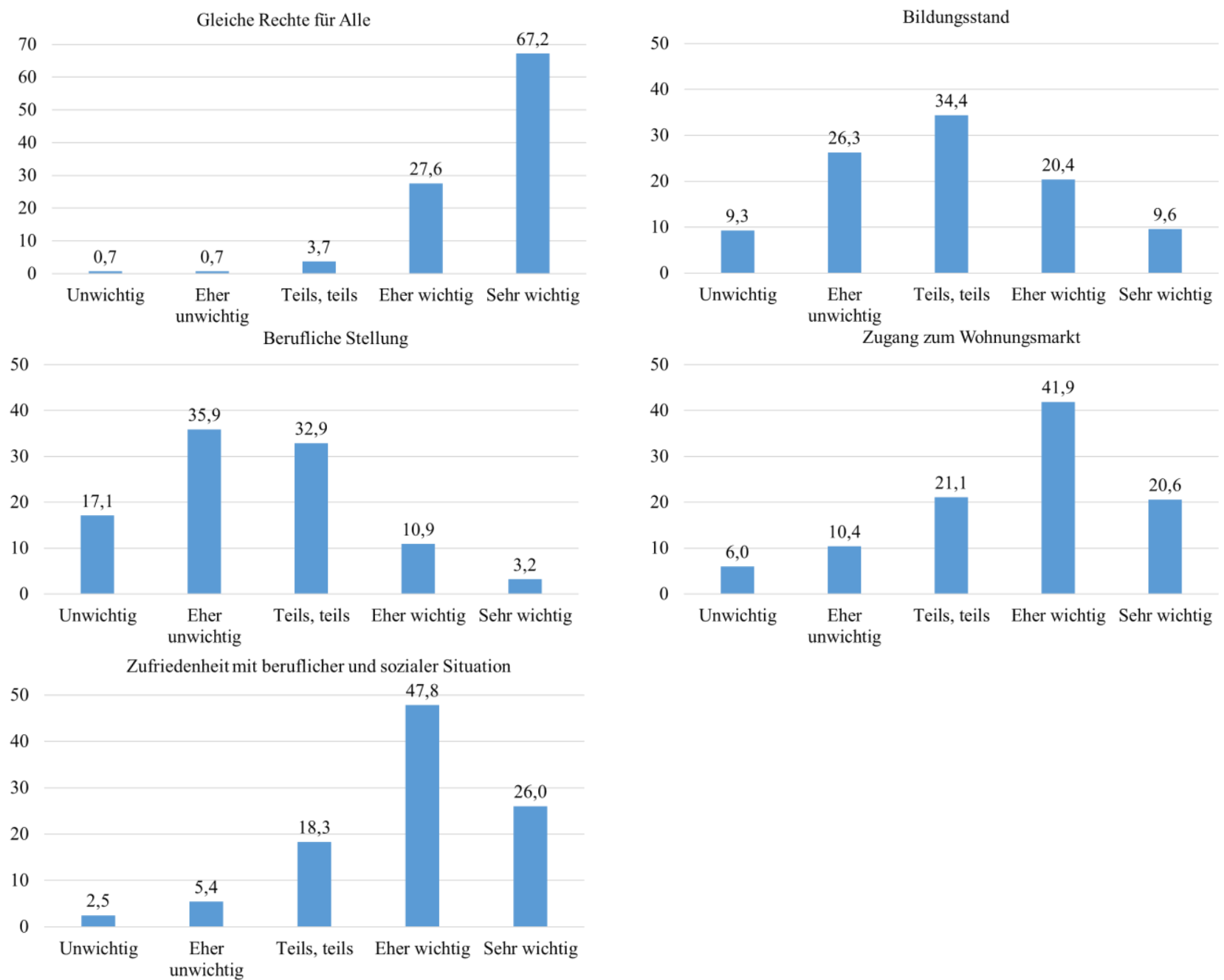

Quelle: Eigene Darstellung; Gleiche Rechte für Alle $n=402$, Bildungsstand $n=407$, Berufliche Stellung $n=404$, Zugang zum Wohnungsmarkt $n=403$, Zufriedenheit mit beruflicher und sozialer Situation $n=404$.

Tabelle 20 berichtet die Mittelwerte der einzelnen Items zu Aspekten von Integration. Hier zeigt sich, dass die abgefragten Aspekte überwiegend positiv bewertet werden und dass vor allem die rechtliche Gleichstellung $(4,60)$, der Wille an der Gesellschaft teilzunehmen $(4,44)$ und Chancen zur Teilnahme an der Gesellschaft $(4,30)$ für die Befragten wichtige Aspekte von Integration darstellen. Darauf folgt die Anerkennung wichtiger Werte $(4,15)$, das Vorhandensein eines Zugehörigkeitsgefühls $(4,15)$ und Kontakte zwischen Menschen unterschiedlicher Kulturen (4,14). Diese erste Hälfte der Aspekte, die alle einen Mittelwert von über vier besitzen, enthält überwiegend Aspekte, die der Dimension der Interaktion zuzurechnen sind. Die weiteren drei Aspekte gehören jeweils einer der anderen Dimensionen (Platzierung, Kulturation, Identifikation) an. In Bezug auf die Akteurinnen und Akteure zeigt sich, dass drei dieser Aspekte Anforderungen an Migrantinnen und Migranten darstellen. Zwei der Aspekte stellen Opportunitäten dar. Demnach lässt sich zunächst keine klare Differenzierung der Bedeutung von Aspekten nach Akteursebene ausmachen. 
Tabelle 20: Mittelwerte der Items zu Aspekten von Integration

\begin{tabular}{|c|c|c|c|c|}
\hline Item & Mittelwert & $\mathbf{N}$ & $\begin{array}{l}\text { Dimension } \\
\text { Integration }\end{array}$ & Akteurin/Akteur \\
\hline Gleiche Rechte für Alle & $4,60(0,67)$ & 402 & Platzierung & Opportunitäten \\
\hline $\begin{array}{l}\text { Der Wille, an der } \\
\text { Gesellschaft teilzunehmen }\end{array}$ & $4,44(0,77)$ & 407 & Interaktion & $\begin{array}{l}\text { Anforderung an } \\
\text { Migrantinnen und } \\
\text { Migranten }\end{array}$ \\
\hline $\begin{array}{l}\text { Chancen zur Teilnahme an } \\
\text { der Gesellschaft }\end{array}$ & $4,30(0,80)$ & 407 & Interaktion & Opportunitäten \\
\hline $\begin{array}{l}\text { Anerkennung wichtiger } \\
\text { Werte }\end{array}$ & $4,15(0,81)$ & 407 & Kulturation & $\begin{array}{l}\text { Anforderung an } \\
\text { Migrantinnen und } \\
\text { Migranten }\end{array}$ \\
\hline $\begin{array}{l}\text { Vorhandensein eines } \\
\text { Zugehörigkeitsgefühls }\end{array}$ & $4,15(0,78)$ & 399 & Identifikation & $\begin{array}{l}\text { Anforderung an } \\
\text { Migrantinnen und } \\
\text { Migranten }\end{array}$ \\
\hline $\begin{array}{l}\text { Kontakte zwischen } \\
\text { Menschen verschiedener } \\
\text { Kulturen }\end{array}$ & $4,14(0,87)$ & 404 & Interaktion & $\begin{array}{l}\text { Anforderung an } \\
\text { Migrantinnen und } \\
\text { Migranten, } \\
\text { Opportunitäten, } \\
\text { Anforderungen an } \\
\text { Gesellschaft }\end{array}$ \\
\hline $\begin{array}{l}\text { Zufriedenheit mit } \\
\text { beruflicher und sozialer } \\
\text { Situation }\end{array}$ & $3,89(0,94)$ & 404 & Platzierung & $\begin{array}{l}\text { Anforderung an } \\
\text { Migrantinnen und } \\
\text { Migranten }\end{array}$ \\
\hline Gute Deutschkenntnisse & $3,87(0,99)$ & 408 & Kulturation & $\begin{array}{l}\text { Anforderung an } \\
\text { Migrantinnen und } \\
\text { Migranten }\end{array}$ \\
\hline $\begin{array}{l}\text { Anerkennung durch } \\
\text { Andere }\end{array}$ & $3,80(0,96)$ & 405 & Interaktion & $\begin{array}{l}\text { Anforderungen an } \\
\text { Gesellschaft }\end{array}$ \\
\hline $\begin{array}{l}\text { Zugang zum } \\
\text { Wohnungsmarkt }\end{array}$ & $3,61(1,10)$ & 403 & Platzierung & Opportunitäten \\
\hline $\begin{array}{l}\text { Mitgliedschaft/Engagement } \\
\text { in Organisationen }\end{array}$ & $3,34(0,99)$ & 406 & Interaktion & $\begin{array}{l}\frac{\text { Anforderung an }}{\text { Migrantinnen und }} \\
\text { Migranten, } \\
\text { Opportunitäten }\end{array}$ \\
\hline Bildungsstand & $2,95(1,11)$ & 407 & Platzierung & $\begin{array}{l}\text { Anforderungen an } \\
\text { Migrantinnen und } \\
\text { Migranten, } \\
\text { Opportunitäten }\end{array}$ \\
\hline Berufliche Stellung & $2,47(1,00)$ & 404 & Platzierung & $\begin{array}{l}\text { Anforderungen an } \\
\text { Migrantinnen und } \\
\text { Migranten, } \\
\text { Opportunitäten }\end{array}$ \\
\hline
\end{tabular}

Quelle: Eigene Darstellung; Anmerkung: Standardabweichung in Klammern.

Betrachtet man die zweite Hälfte der Aspekte mit einem Mittelwert von unter vier, zeigt sich zunächst, dass der Aspekt der Deutschkenntnisse lediglich einen Mittelwert von 3,87 aufweist und diesem Aspekt hier somit nicht die gleiche Bedeutung wie in der bisherigen politischen 
und gesellschaftlichen Diskussion zukommt. Gleiches gilt für den Bildungsstand und die berufliche Stellung. Die Aspekte Bildungsstand $(2,95)$ und Berufliche Stellung $(2,47)$ werden insgesamt als am unwichtigsten für Integration bewertet. Gleichzeitig liegt deren Mittelwert unter dem Wert der Mittelkategorie (3,00). Bei Betrachtung der Akteursebenen zeigt sich eine ähnliche Verteilung von Anforderungen an Migrantinnen und Migranten sowie Opportunitäten in der oberen und unteren Tabellenhälfte. Darüber hinaus ist diese zweite Hälfte der Items stark durch die Dimension der Platzierung geprägt. Vier der fünf Items der Platzierung befinden sich in dieser Hälfte der Tabelle. Bei der Dimension der Interaktion sind es hingegen nur zwei von fünf Items. In ihrer Bedeutung scheint die Dimension der Platzierung somit der Dimension der Interaktion untergeordnet zu sein. Für die anderen Dimensionen kann aufgrund der geringen Zahl von Items keine Unterscheidung stattfinden. Dennoch liegen die Gesamtmittelwerte der Aspekte, die den Dimensionen Kulturation und Identifikation zugerechnet werden, über dem Gesamtmittelwert der Platzierung. Somit können zum einen die Dimensionen als auch die Aspekte innerhalb der Dimensionen in der ihnen zugemessenen Bedeutung für Integration dargestellt werden.

Betrachtet man die Ausgänge von Integration zeigen sich Unterschiede in der Zustimmung zu den einzelnen Items (vgl. Abbildung 8). Dem Ausgang der Segregation, der als „Wenn Zuwanderer ihre Kultur beibehalten möchten, sollten sie unter sich bleiben“ formuliert wurde, wird überwiegend nicht zugestimmt. 39,9\% der Befragten stimmen diesem Item gar nicht und $36,5 \%$ eher nicht zu. Lediglich 3,4\% stimmen diesem Item (eher) zu. Demnach stellt die Segregation bei den Befragten keinen wünschenswerten Ausgang von Integration dar.

In Bezug auf pluralistische Tendenzen, die im Fragebogen als „Es wäre gut, wenn alle Zuwanderer in Deutschland ihre Kultur beibehielten“ formuliert wurden, zeigt sich ein eher durchmischtes Bild. Die meisten Befragten geben hier „Teils, teils“ an. Über die Mittelkategorie hinaus zeigt sich eher eine Ablehnung dieses Konzepts (23,8 \%). Nur 18,3\% der Befragten stimmen diesem Konzept (eher) zu. Eine vollständige Beibehaltung der Kultur der Zugewanderten wird von den Befragten somit überwiegend abgelehnt oder die Befragten beziehen keine eindeutige Stellung.

Das klare Gegenteil zeigt sich bei assimilativen Ansichten. Diese wurden im Fragebogen mit Hilfe der Zustimmung zum Item „Menschen, die nach Deutschland kommen, sollten ihr Verhalten der deutschen Kultur anpassen“ gemessen. Insgesamt geben auch hier die meisten Befragten „Teils, teils“ an (42,7 \%). Jedoch stimmen gleichzeitig auch 34,9 \% der Formulierung (eher) zu und nur 12,4\% stimmen dieser Aussage (eher) nicht zu. 
Am positivsten bewertet wird der Ausgang der Mehrfachintegration (,Zuwanderer sollten sowohl in ihre Herkunftsgesellschaft als auch in die deutsche Gesellschaft eingebunden sein"). Insgesamt 83,1 \% der Befragten stimmen diesem Item (eher) zu, während 4,1\% (eher) nicht zustimmen. Gleichzeitig zeigt sich bei dieser Aussage auch eine deutlich geringere Besetzung der Mittelkategorie (12,7 \%) als bei den anderen aufgeführten Integrationskonzepten. Während vor allem bei der Aussage zu Pluralismus und Assimilation somit größere Unsicherheit durch eine starke Besetzung der Mittelkategorie herrscht, stimmen die Befragten dem Item zur Mehrfachintegration deutlich zu und lehnen das Item zur Segregation deutlich ab.

Abbildung 8: Verteilung der Items zu Ausgängen von Integration in Prozent
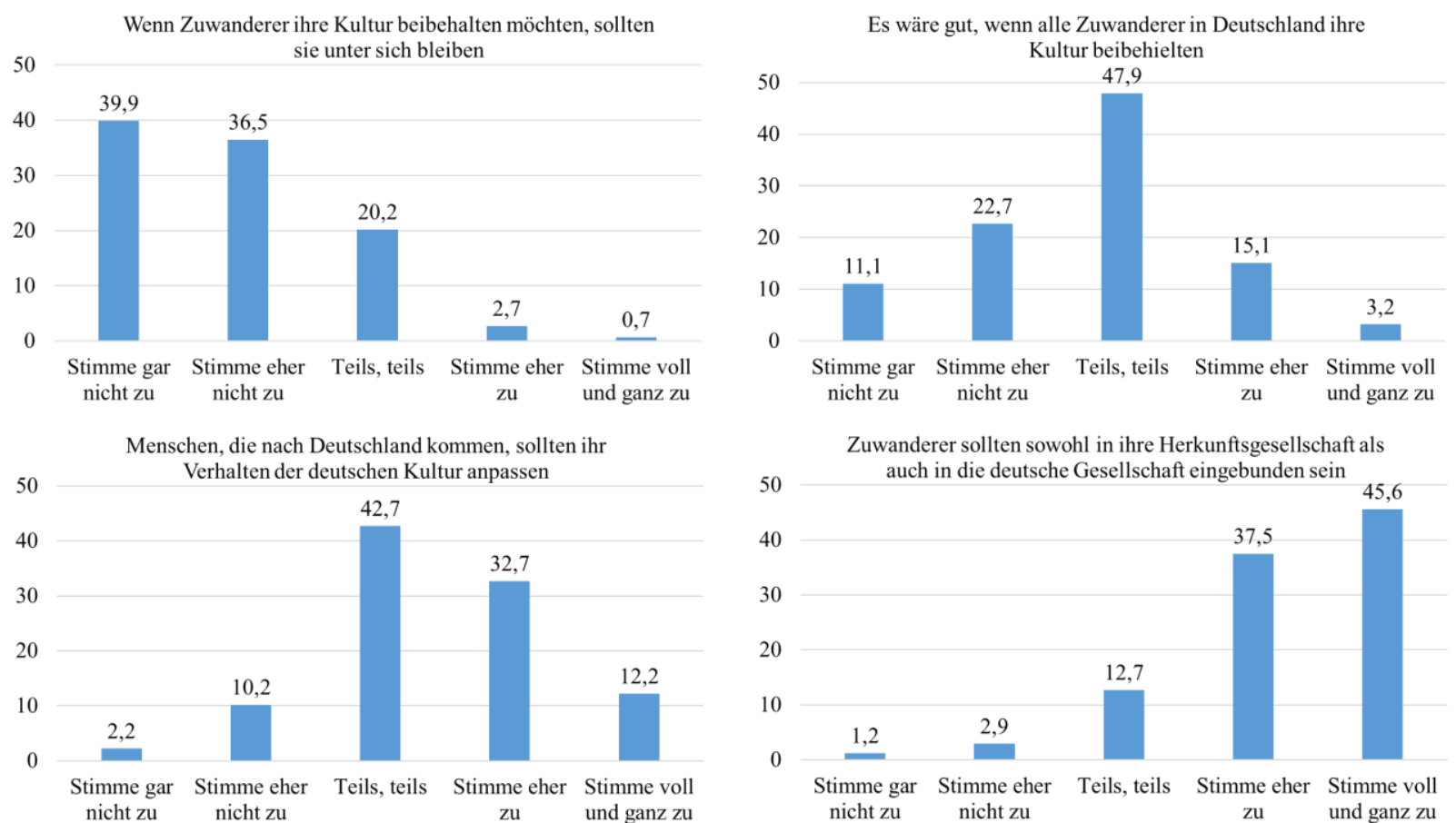

Quelle: Eigene Darstellung; „Wenn Zuwanderer ihre Kultur beibehalten möchten, sollten sie unter sich bleiben“ $\mathrm{n}=406$, „Es wäre gut, wenn alle Zuwanderer in Deutschland ihre Kultur beibehielten“ $n=405$, „Menschen, die nach Deutschland kommen, sollten ihr Verhalten der deutschen Kultur anpassen“ $n=410$, „Zuwanderer sollten sowohl in ihre Herkunftsgesellschaft als auch in die deutsche Gesellschaft eingebunden sein“ $n=408$.

Insgesamt zeigt die Analyse zu Ausgängen von Integration, dass vor allem der Ausgang der Mehrfachintegration favorisiert wird. Dennoch spielen auch assimilative Tendenzen eine Rolle und gerade darüber, ob Zugewanderte ihre Kultur beibehalten sollten, herrscht überwiegend Unsicherheit. Zumindest konnte in Bezug auf dieses Item keine Mehrheit in der Ablehnung oder Zustimmung ausgemacht werden, da überwiegend die Mittelkategorie angegeben wurde. Darüber hinaus sollten Zugewanderte nicht unter sich bleiben, selbst wenn sie ihre Kultur beibehalten möchten. Die Segregation wird somit als Ausgang von Integration am ehesten abgelehnt, während sich bei den wünschenswerten Ausgängen eine Mischung aus 
Mehrfachintegration, als eine Ausgestaltung von pluralistischen Ansichten, und assimilativen Tendenzen zeigt. Dabei bleibt jedoch unklar, inwieweit Zugewanderte ihre Kultur beibehalten sollten. Da dieses Item jedoch schlechter bewertet wird als das Item zur Assimilation, steht der Prozess der Anpassung wohl im Vordergrund. Diese Ergebnisse werden in der nachfolgenden Tabelle 21 nochmals mit Hilfe der Mittelwerte der einzelnen Items dargestellt. Die Mehrfachintegration weist dabei den höchsten Mittelwert von 4,23 auf. Darauf folgt das Item zur Assimilation mit 3,42. Das Item zum Pluralismus in der Ausgestaltung, dass alle Zugewanderten ihre Kultur beibehalten sollten sowie die Segmentation weisen Mittelwerte auf, die unter dem Wert der mittleren Antwortkategorie liegen und werden somit eher abgelehnt. Beim Item zum Pluralismus liegt der Mittelwert bei 2,77 und beim Item zur Segmentation bei 1,88 .

Tabelle 21: Mittelwerte der Items zu Zielen von Integration

\begin{tabular}{l|l|l|l|}
\hline Item & \multicolumn{1}{|c|}{ Mittelwert } & N & Konzept \\
\hline $\begin{array}{l}\text { Wenn Zuwanderer ihre Kultur } \\
\text { beibehalten möchten, sollten sie unter sich } \\
\text { bleiben }\end{array}$ & $1,88(0,87)$ & 406 & Segmentation \\
\hline $\begin{array}{l}\text { Es wäre gut, wenn alle Zuwanderer in } \\
\text { Deutschland ihre Kulturen beibehielten }\end{array}$ & $2,77(0,95)$ & 405 & Pluralismus \\
\hline $\begin{array}{l}\text { Menschen, die nach Deutschland kommen, } \\
\text { sollten ihr Verhalten der deutschen Kultur } \\
\text { anpassen }\end{array}$ & $3,42(0,91)$ & 410 & Assimilation \\
\hline $\begin{array}{l}\text { Zuwanderer sollten sowohl in ihre } \\
\text { Herkunftsgesellschaft als auch in die } \\
\text { deutsche Gesellschaft eingebunden sein }\end{array}$ & & 408 & Mehrfachintegration \\
\hline
\end{tabular}

Quelle: Eigene Darstellung; Anmerkung: Standardabweichung in Klammern. 


\subsubsection{Analyse von Unterschieden zwischen Vereinsmitgliedern und Vorständen}

Innerhalb dieses Unterkapitels werden Unterschiede zwischen Vereinsmitgliedern und Vorständen in Bezug auf die Bewertung von Aspekten und Zielen von Integration dargestellt. Die Unterscheidung zwischen diesen Personengruppen ist innerhalb der Vereine relevant, da diese Gruppen unterschiedliche Einflüsse auf die Ausgestaltung von Programmen wie beispielsweise Integrationsprojekten haben. Demnach könnten vor allem die Ansichten der Vorstände diese Programme färben, sofern es Unterschiede in der Sichtweise auf Integration zwischen diesen Gruppen gibt. Für die Analyse der Unterschiede wurden t-Tests sowie MannWhitney U-Tests durchgeführt, deren Ergebnisse in der nachfolgenden Tabelle 22 dargestellt werden.

Beim Vergleich der Bewertungen durch Vereinsmitglieder und Vorstände zeigt sich bei den Aspekten von Integration lediglich ein signifikanter Unterschied beim Item „Mitgliedschaft/Engagement in Organisationen“. In Bezug auf die Ausgänge von Integration zeigen sich hingegen keine signifikanten Unterschiede. Auffällig ist hierbei, dass es sich beim Item zu Mitgliedschaft und Engagement in Organisationen um das einzige Item handelt, das einen Bezug zum Sportverein als Organisation aufweist. Alle anderen Aspekte, die auch konkreter mit Integration in Bezug stehen, zeigen jedoch keine signifikanten Unterschiede in ihrer Bewertung durch Vereinsvorstände und Mitglieder.

Tabelle 22: Ergebnisse der t-Tests und U-Tests zur Bewertung von Aspekten und Zielen von Integration von Vereinsmitgliedern und Vorständen

Item

Mittelwert Mittelwert t-Test U-Test

Vorstand Mitglieder

(z)

\begin{tabular}{|c|c|c|c|c|}
\hline Gute Deutschkenntnisse V & $4,00(0,83)$ & $3,83(1,02)$ & $-1,394$ & $-1,007$ \\
\hline Anerkennung wichtiger Werte & $4,11(0,77)$ & $4,15(0,82)$ & 0,460 & $-0,776$ \\
\hline $\begin{array}{l}\text { Chancen zur Teilnahme an der } \\
\text { Gesellschaft }\end{array}$ & $4,22(0,77)$ & $4,32(0,81)$ & 1,090 & $-1,457$ \\
\hline $\begin{array}{l}\text { Der Wille, an der Gesellschaft } \\
\text { teilzunehmen }\end{array}$ & $4,41(0,74)$ & $4,45(0,77)$ & 0,333 & $-0,535$ \\
\hline $\begin{array}{l}\text { Kontakte zwischen Menschen } \\
\text { verschiedener Kulturen }\end{array}$ & $4,18(0,80)$ & $4,13(0,89)$ & $-0,489$ & $-0,299$ \\
\hline $\begin{array}{l}\text { Mitgliedschaft/ } \\
\text { Engagement in Organisationen }\end{array}$ & $3,59(0,86)$ & $3,28(1,01)$ & $-2,561 *$ & $-2,248 *$ \\
\hline Anerkennung durch Andere & $3,90(0,92)$ & $3,78(0,97)$ & $-1,074$ & $-0,949$ \\
\hline $\begin{array}{l}\text { Vorhandensein eines } \\
\text { Zugehörigkeitsgefühls }\end{array}$ & $4,29(0,69)$ & $4,11(0,80)$ & $-1,864$ & $-1,791$ \\
\hline Gleiche Rechte für Alle & $4,60(0,58)$ & $4,60(0,69)$ & $-0,082$ & $-0,385$ \\
\hline Bildungsstand $^{v}$ & $3,07(0,99)$ & $2,91(1,13)$ & $-1,167$ & $-1,259$ \\
\hline
\end{tabular}




\begin{tabular}{|c|c|c|c|c|}
\hline Berufliche Stellung & $2,4(0,96)$ & $2,43(1,01)$ & $-1,695$ & $-1,635$ \\
\hline Zugang zum Wohnungsmarkt & $3,76(1,14)$ & $3,57(1,09)$ & $-1,363$ & $-1,697$ \\
\hline $\begin{array}{l}\text { Zufriedenheit mit beruflicher und } \\
\text { sozialer Situation } v\end{array}$ & $4,02(0,87)$ & $3,86(0,95)$ & $-1,413$ & $-1,498$ \\
\hline $\begin{array}{l}\text { Wenn Zuwanderer ihre Kultur } \\
\text { beibehalten möchten, sollten sie unter } \\
\text { sich bleiben }\end{array}$ & $1,85(0,86)$ & $1,89(0,88)$ & 0,297 & $-0,332$ \\
\hline $\begin{array}{l}\text { Es wäre gut, wenn alle Zuwanderer in } \\
\text { Deutschland ihre Kulturen beibehielten }\end{array}$ & $2,80(0,93)$ & $2,76(0,95)$ & $-0,393$ & $-0,249$ \\
\hline $\begin{array}{l}\text { Menschen, die nach Deutschland } \\
\text { kommen, sollten ihr Verhalten der } \\
\text { deutschen Kultur anpassen }\end{array}$ & $3,43(0,93)$ & $3,42(0,91)$ & $-0,027$ & $-0,104$ \\
\hline $\begin{array}{l}\text { Zuwanderer sollten sowohl in ihre } \\
\text { Herkunftsgesellschaft als auch in die } \\
\text { deutsche Gesellschaft eingebunden sein }\end{array}$ & $4,11(0,91)$ & $4,26(0,86)$ & 1,460 & $-1,563$ \\
\hline
\end{tabular}

Insgesamt ergibt sich beim Item zu Mitgliedschaft/Engagement in Organisationen zwischen Mitgliedern und Vorständen ein Mittelwertunterschied von 0,31. Abbildung 9 zeigt die Verteilungen des Items. Diese zeigt, dass die Mitglieder dieses Item mit 19,2 \% als (eher) unwichtig bewerten, während nur 7,2 \% der Vorstände eher unwichtig und $0 \%$ unwichtig angeben. Diese Einschätzung ergibt sich voraussichtlich aus der eigenen Position im Verein und auch daraus, dass Vorstände bereits eine aktive Position im Verein besitzen und aus diesem Grund dieses Engagement auch als wichtiger einstufen als die Mitglieder.

Abbildung 9: Verteilung des Items Mitgliedschaft/Engagement in Organisationen in Prozent nach Vereinsmitgliedern und Vorständen

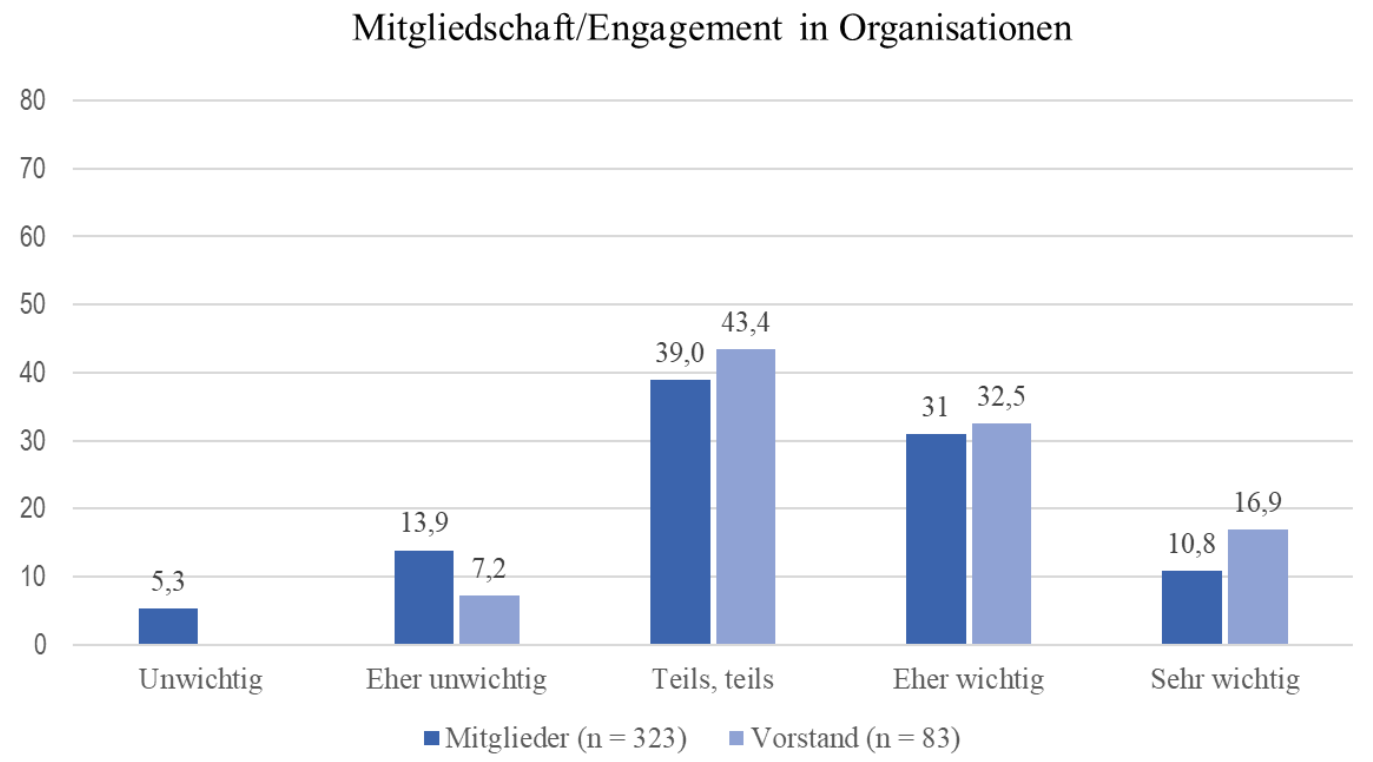

Quelle: Eigene Darstellung. 


\subsubsection{Analyse von Unterschieden zwischen Personen mit und ohne}

\section{Migrationshintergrund}

Im Folgenden werden Unterschiede in der Bewertung von Aspekten und Zielen von Integration zwischen Personen mit und ohne Migrationshintergrund dargestellt. Diese Unterscheidung ist für die vorliegende Arbeit von Interesse, da sich bereits im Forschungsstand Unterschiede zwischen diesen beiden Gruppen gezeigt haben. Vor allem vor dem Hintergrund der eigenen Erfahrung könnten bestimmte Aspekte und Ziele von Integration als bedeutender bewertet werden. Wie im Kapitel zu den Analyseverfahren dargestellt, wurden t-Tests sowie MannWhitney U-Tests durchgeführt, um Unterschiede in der Bewertung von Aspekten und Zielen von Integration zu untersuchen. In der nachfolgenden Tabelle 23 sind die Ergebnisse dieser Tests dargestellt.

Betrachtet man die beiden Tests, zeigt sich zunächst, dass diese bis auf eine Ausnahme, die gleichen signifikanten Unterschiede in den bewerteten Items aufweisen. Signifikante Unterschiede in der Bewertung von Aspekten von Integration von Personen mit und ohne Migrationshintergrund zeigen sich in Bezug auf Chancen zur Teilnahme an der Gesellschaft und dem Item Gleiche Rechte für Alle. Für das Item Kontakte zwischen Menschen unterschiedlicher Kulturen weist nur der U-Test ein signifikantes Ergebnis auf, welcher wegen der ungleichen Gruppengrößen, fehlender Normalverteilung und ungleicher Varianzen der untersuchten Variable in diesem Fall herangezogen wird. Bei den Ergebnissen von Integration zeigt sich hingegen nur beim Item „Es wäre gut, wenn alle Zuwanderer in Deutschland ihre Kulturen beibehielten“ ein signifikanter Unterschied zwischen Personen mit und ohne Migrationshintergrund. Insgesamt bleibt außerdem festzuhalten, dass die Unterschiede zwischen Personen mit und ohne Migrationshintergrund eher gering ausfallen. 
Tabelle 23: Ergebnisse der t-Tests und U-Tests zur Bewertung von Aspekten und Zielen von Integration von Personen mit und ohne Migrationshintergrund

Item

Mittelwert Mittelwert t-Test U-Test

ohne MGH mit MGH

(z)

\begin{tabular}{|c|c|c|c|c|}
\hline Gute Deutschkenntnisse & $3,86(0,99)$ & $3,88(0,99)$ & $-0,160$ & $-0,184$ \\
\hline Anerkennung wichtiger Werte & $4,14(0,82)$ & $4,16(0,78)$ & $-0,154$ & $-0,125$ \\
\hline $\begin{array}{l}\text { Chancen zur Teilnahme an der } \\
\text { Gesellschaft }\end{array}$ & $4,26(0,79)$ & $4,47(0,81)$ & $-2,081 *$ & $-2,648 * *$ \\
\hline $\begin{array}{l}\text { Der Wille, an der Gesellschaft } \\
\text { teilzunehmen }\end{array}$ & $4,43(0,77)$ & $4,49(0,74)$ & $-0,593$ & $-0,702$ \\
\hline $\begin{array}{l}\text { Kontakte zwischen Menschen } \\
\text { verschiedener Kulturen }{ }^{v}\end{array}$ & $4,10(0,85)$ & $4,32(0,93)$ & $-1,852$ & $-2,551 *$ \\
\hline $\begin{array}{l}\text { Mitgliedschaft/ } \\
\text { Engagement in Organisationen }\end{array}$ & $3,34(0,98)$ & $3,37(1,04)$ & $-0,277$ & $-0,313$ \\
\hline Anerkennung durch Andere & $3,77(0,98)$ & $3,96(0,88)$ & $-1,573$ & $-1,320$ \\
\hline $\begin{array}{l}\text { Vorhandensein eines } \\
\text { Zugehörigkeitsgefühls }\end{array}$ & $4,12(0,80)$ & $4,27(0,68)$ & $-1,438$ & $-1,285$ \\
\hline Gleiche Rechte für Alle ${ }^{V}$ & $4,56(0,70)$ & $4,75(0,52)$ & $-2,656^{*}$ & $-2,372 *$ \\
\hline Bildungsstand & $2,92(1,09)$ & $3,07(1,16)$ & $-1,047$ & $-0,723$ \\
\hline Berufliche Stellung & $2,46(0,99)$ & $2,54(1,06)$ & $-0,644$ & $-0,525$ \\
\hline Zugang zum Wohnungsmarkt & $3,58(1,09)$ & $3,74(1,17)$ & $-1,130$ & $-1,440$ \\
\hline $\begin{array}{l}\text { Zufriedenheit mit beruflicher und } \\
\text { sozialer Situation }\end{array}$ & $3,87(0,95)$ & $4,01(0,86)$ & $-1,238$ & $-1,138$ \\
\hline $\begin{array}{l}\text { Wenn Zuwanderer ihre Kultur } \\
\text { beibehalten möchten, sollten sie unter } \\
\text { sich bleiben }\end{array}$ & $1,91(0,87)$ & $1,75(0,90)$ & 1,433 & $-1,700$ \\
\hline $\begin{array}{l}\text { Es wäre gut, wenn alle Zuwanderer in } \\
\text { Deutschland ihre Kulturen beibehielten }\end{array}$ & $2,71(0,94)$ & $3,01(0,93)$ & $-2,546 *$ & $-2,147^{*}$ \\
\hline $\begin{array}{l}\text { Menschen, die nach Deutschland } \\
\text { kommen, sollten ihr Verhalten der } \\
\text { deutschen Kultur anpassen }\end{array}$ & $3,44(0,90)$ & $3,38(0,93)$ & 0,511 & $-0,209$ \\
\hline $\begin{array}{l}\text { Zuwanderer sollten sowohl in ihre } \\
\text { Herkunftsgesellschaft als auch in die } \\
\text { deutsche Gesellschaft eingebunden sein }\end{array}$ & $4,22(0,87)$ & $4,30(0,87)$ & $-0,736$ & $-0,898$ \\
\hline
\end{tabular}

Quelle: Eigene Darstellung; Anmerkungen: Standardabweichung in Klammern, V= Ungleiche Varianzen, ${ }^{*} \mathrm{p}<$ 0,$05 ; * * \mathrm{p}<0,01$.

Es zeigt sich auch, dass das Item Gleiche Rechte für Alle im Vergleich der Mittelwerte sowohl für Personen mit als auch ohne Migrationshintergrund, den am höchsten bewerteten Aspekt darstellt. Darauf folgen die Chancen und der Wille zur Teilnahme an der Gesellschaft.

Betrachtet man das Item Chancen zur Teilnahme an der Gesellschaft zeigt sich zwischen Personen mit und ohne Migrationshintergrund zunächst ein Unterschied der Mittelwerte von 
0,21. Personen ohne Migrationshintergrund weisen in Bezug auf dieses Item einen Mittelwert von 4,26 und Personen mit Migrationshintergrund von 4,47 auf. Im Vergleich zu den anderen beiden Items, deren Bewertung sich zwischen diesen Gruppen signifikant unterscheidet, weist dieses Item somit den größten Unterschied auf. Die Verteilung dieses Items wird in der nachfolgenden Abbildung 10 dargestellt.

Abbildung 10: Verteilung des Items Chancen zur Teilnahme an der Gesellschaft in Prozent nach Migrationshintergrund

Chancen zur Teilnahme an der Gesellschaft

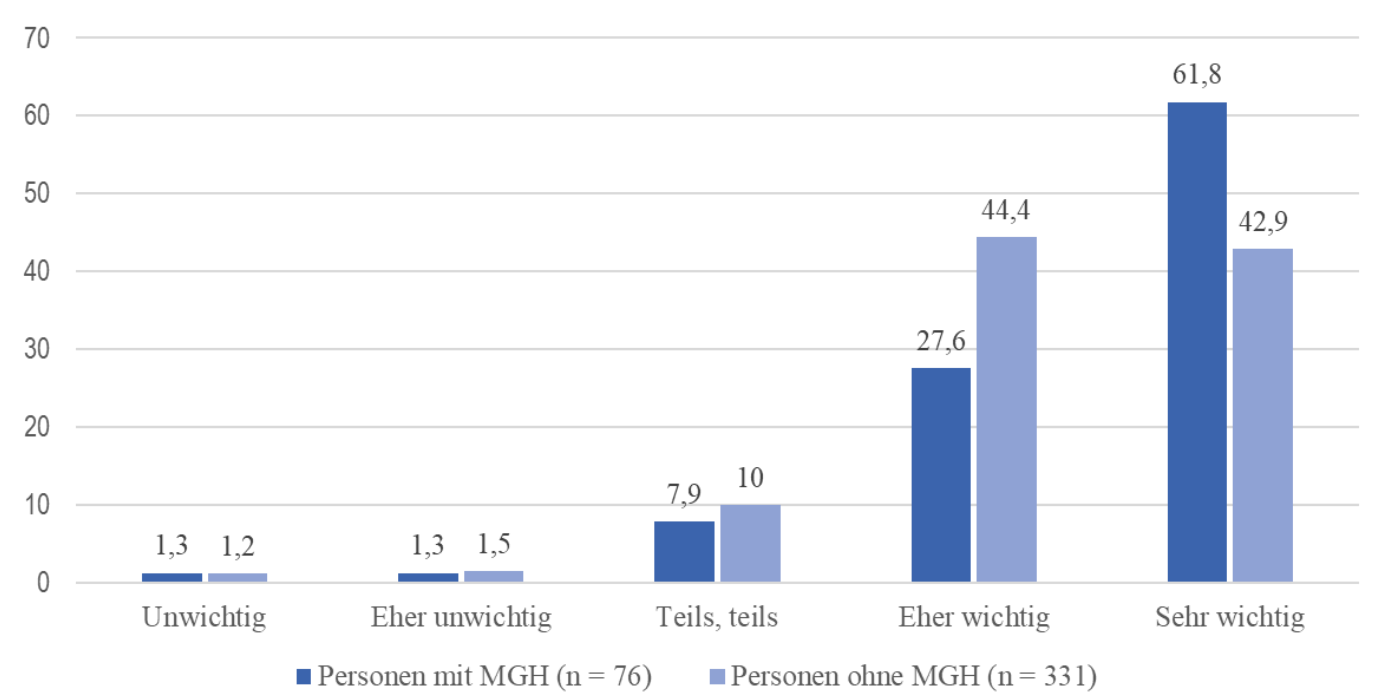

Quelle: Eigene Darstellung.

Es zeigt sich, dass die Unterschiede zwischen Personen mit und ohne Migrationshintergrund überwiegend in der höheren Bewertung dieses Items bestehen. 61,8\% der Personen mit Migrationshintergrund bewerten dieses Item als sehr wichtig, während es bei Personen ohne Migrationshintergrund nur 42,9 \% sind. Dies könnte auf unterschiedliche Erfahrungen in Bezug auf beispielsweise Zugänge in Form von Chancen zur Teilnahme an der Gesellschaft zurückzuführen sein.

Gleiches zeigt sich auch bei Betrachtung der Verteilungen des Items zu Kontakten zwischen Menschen verschiedener Kulturen (Abbildung 11). Zunächst zeigt sich bei diesem Item ein Mittelwertunterschied von 0,22. Während hier mehr als die Hälfte (56,6 \%) der Personen mit Migrationshintergrund interethnische Kontakte als sehr wichtig bewerten, sind es bei den Personen ohne Migrationshintergrund 34,6\%. Der Großteil der Personen ohne Migrationshintergrund bewertet diese interethnischen Kontakte zudem als eher wichtig $(46,6 \%)$. 
Abbildung 11: Verteilung des Items Kontakte zwischen Menschen verschiedener Kulturen in Prozent nach Migrationshintergrund

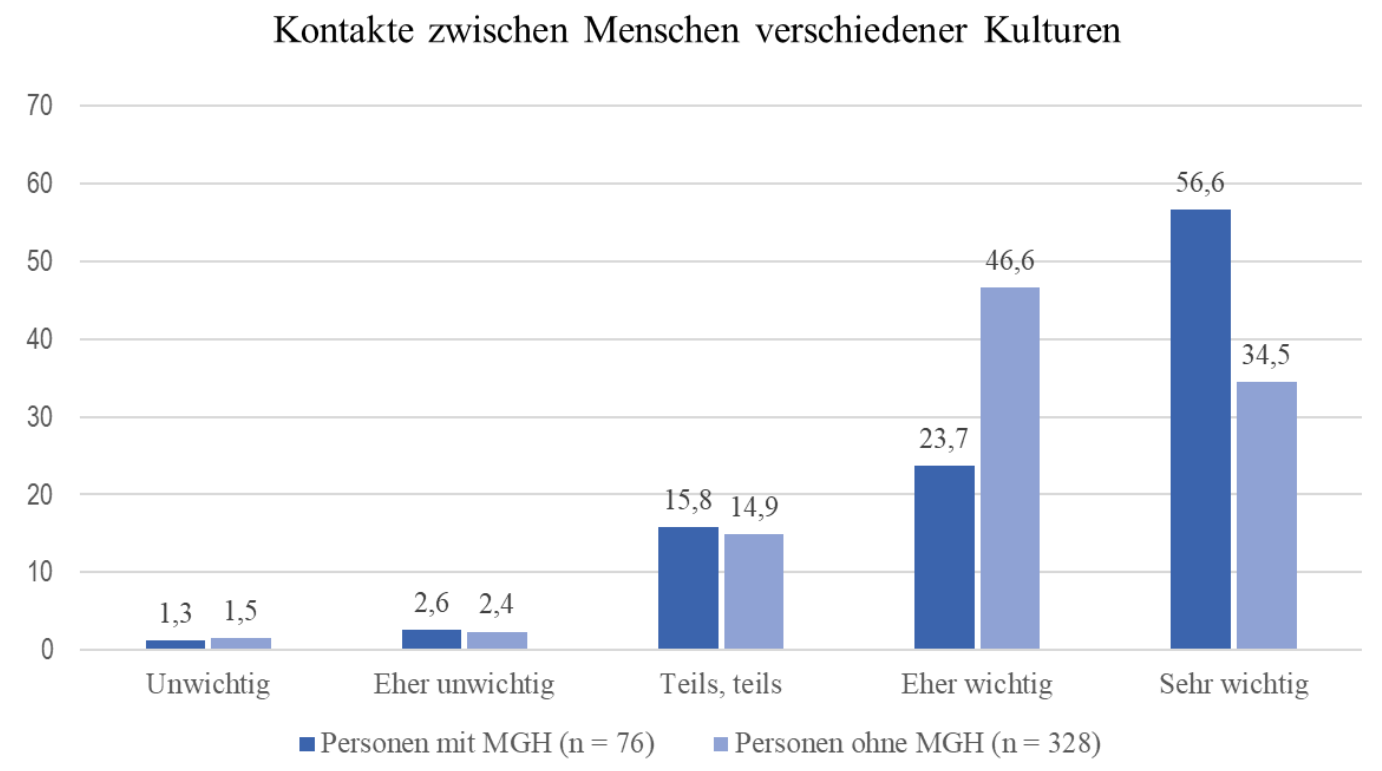

Quelle: Eigene Darstellung.

Der letzte Aspekt, für den signifikante Unterschiede zwischen Personen mit und ohne Migrationshintergrund signifikant vorliegen, bezieht sich auf die rechtliche Gleichstellung. Hier liegt der Mittelwertunterschied zwischen den Gruppen bei 0,19. Bei diesem Item zeigt sich ebenfalls ein Unterschied in Bezug auf die Besetzung der Kategorie „Sehr wichtig“ (vgl. Abbildung 12). Bei Personen mit Migrationshintergrund stufen 78,9\% der Befragten diesen Aspekt als sehr wichtig ein, wohingegen es bei Personen ohne Migrationshintergrund 64,4 \% sind. Dennoch ist anzumerken, dass dieses Item von beiden Gruppen mit einem Anteil von mehr als der Hälfte der Befragten als sehr wichtig eingestuft wird. Auffällig ist darüber hinaus, dass jeweils $0,9 \%$ der Personen ohne Migrationshintergrund dieses Item auch als unwichtig oder eher unwichtig einstufen. Hingegen stufte keiner der Befragten mit Migrationshintergrund das Item als unwichtig ein. 
Abbildung 12: Verteilung des Items Gleiche Rechte für Alle in Prozent nach Migrationshintergrund

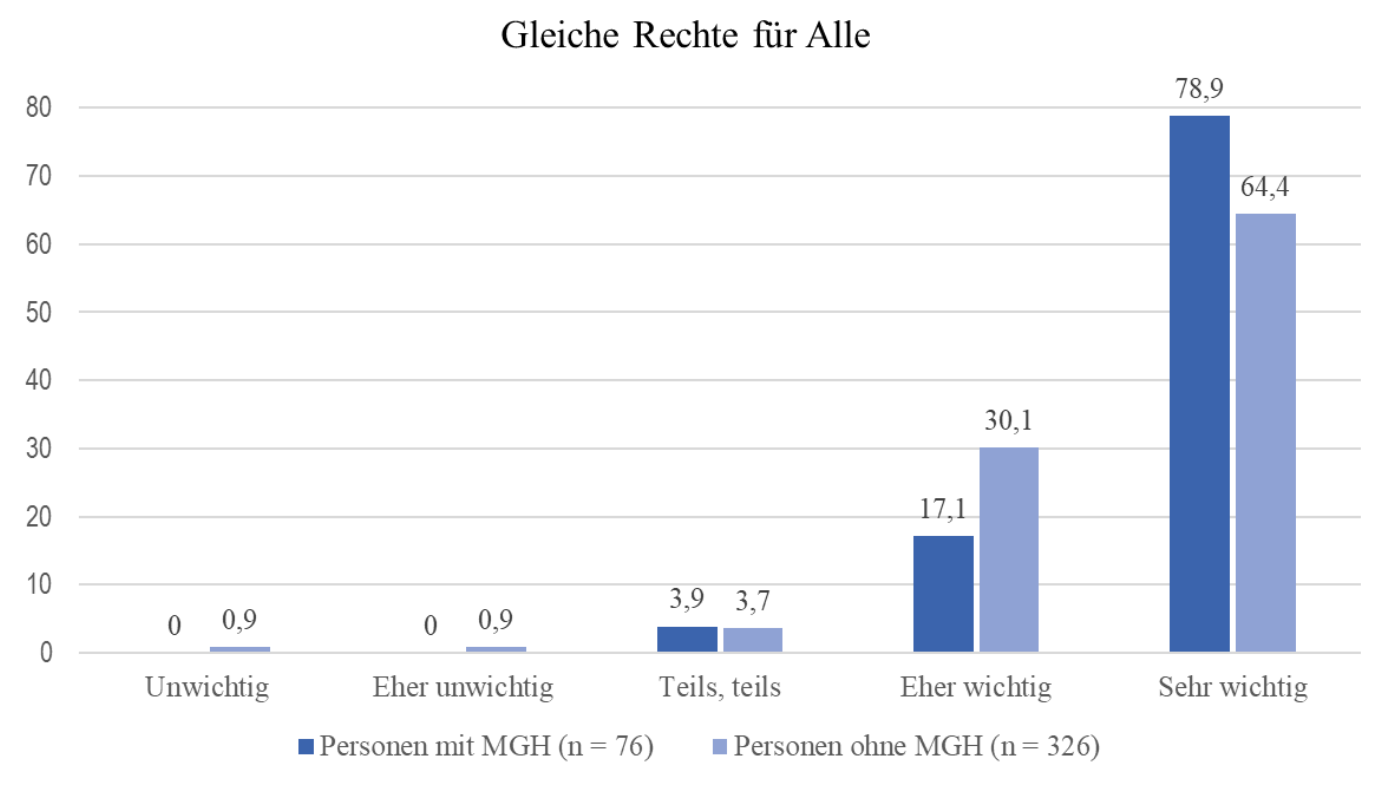

Quelle: Eigene Darstellung.

Neben signifikanten Unterschieden bei der Bewertung der Aspekte von Integration, zeigt sich auch ein signifikanter Unterschied in der Bewertung der Aussage „Es wäre gut, wenn alle Zuwanderer in Deutschland ihre Kulturen beibehielten“, welches einen pluralistischen Ausgang von Integration darstellt, bei dem eine Beibehaltung der Kultur des Herkunftslandes angestrebt wird. Der Mittelwertunterschied zwischen den Gruppen liegt bei diesem Item bei 0,30 und Personen mit Migrationshintergrund stimmen der Aussage stärker zu. Die Verteilung des Items für beide Gruppen ist in Abbildung 13 dargestellt. Es zeigen sich Unterschiede in der Antwortkategorie „Stimme gar nicht zu“ und „Stimme voll und ganz zu“. Während 3,9 \% der Personen mit Migrationshintergrund dieser Aussage gar nicht zustimmen, sind es bei den Personen ohne Migrationshintergrund 12,9\%. Gleichzeitig zeigt sich bei den Personen mit Migrationshintergrund auch eine größere Zustimmung $\mathrm{zu}$ diesem Item innerhalb der Antwortkategorie „Stimme voll und ganz $\mathrm{zu}^{\text {“ }}(7,9 \%)$ als bei Personen ohne Migrationshintergrund. Bei Personen ohne Migrationshintergrund liegt der Anteil hier nur bei $2,1 \%$. 
Abbildung 13: Verteilung des Items zu Pluralismus nach Migrationshintergrund

Es wäre gut, wenn alle Zuwanderer in Deutschland ihre Kulturen beibehielten

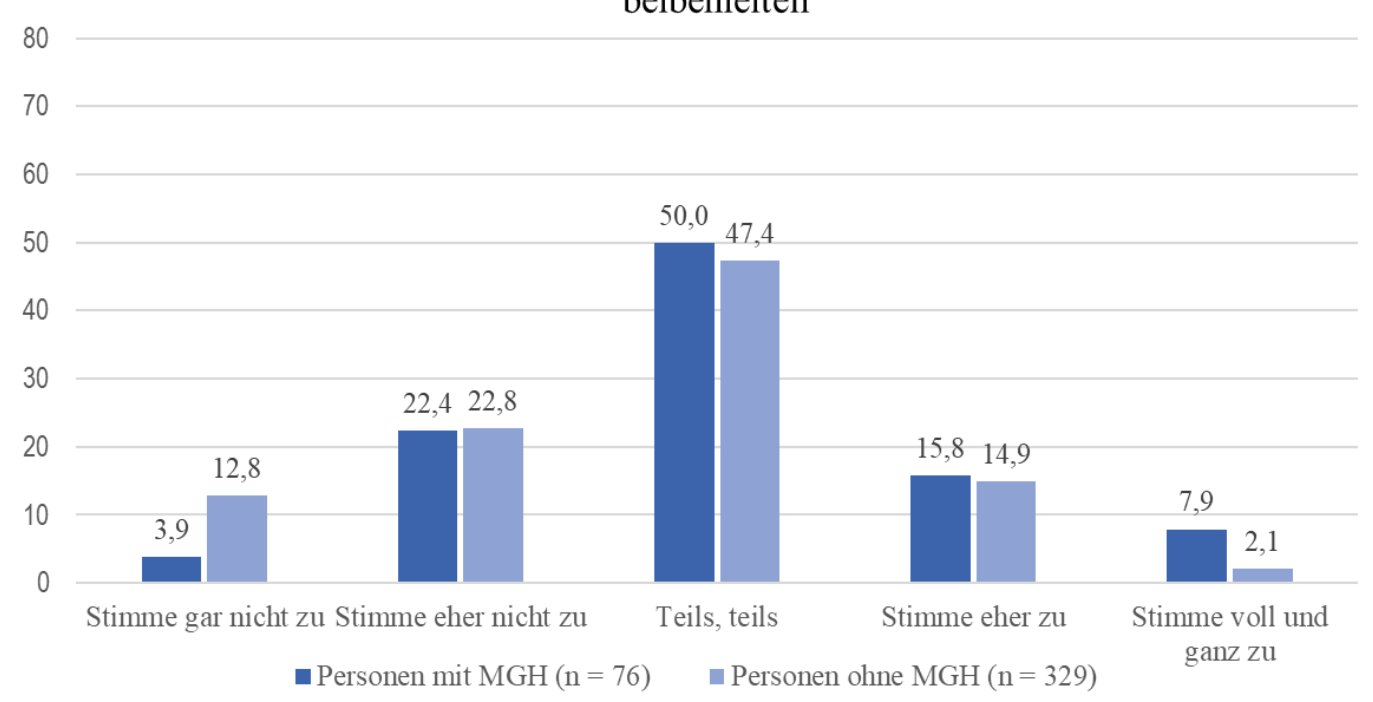

Quelle: Eigene Darstellung.

Neben den Unterschieden zwischen Personen mit und ohne Migrationshintergrund konnten außerdem Analysen für Unterschiede zwischen Migrantinnen und Migranten erster und zweiter Generation durchgeführt werden, deren Ergebnisse in Tabelle 24 dargestellt werden. Anzumerken ist hierbei, dass beide Gruppen nur einen geringen Umfang aufweisen. So enthält die Stichprobe 30 Migrantinnen und Migranten erster sowie 46 Migrantinnen und Migranten zweiter Generation. Signifikante Unterschiede in der Bewertung von Aspekten und Ausgängen von Integration zeigen sich demnach bezüglich der Items zu Kontakten zwischen Menschen verschiedener Kulturen und dem Vorhandensein eines Zugehörigkeitsgefühls. Das Item Anerkennung wichtiger Werte weist lediglich beim t-Test einen signifikanten Unterschied zwischen Migrantinnen und Migranten erster und zweiter Generation auf. Aufgrund der geringen Gruppengrößen und der Vergleichbarkeit mit den vorangegangenen Auswertungen wird jedoch auch hier dem U-Test Vorrang gewährt. 
Tabelle 24: Ergebnisse der t-Tests und U-Tests zur Bewertung von Aspekten und Zielen von Integration von Migrantinnen und Migranten erster und zweiter Generation

Item

Mittelwert Mittelwert t-Test U-Test (z)

1. Gen. 2. Gen.

\begin{tabular}{|c|c|c|c|c|}
\hline Gute Deutschkenntnisse & $3,73(1,08)$ & $3,98(0,93)$ & $-1,052$ & $-0,934$ \\
\hline Anerkennung wichtiger Werte ${ }^{V}$ & $3,90(1,03)$ & $4,32(0,52)$ & $-2,101 *$ & $-1,716$ \\
\hline $\begin{array}{l}\text { Chancen zur Teilnahme an der } \\
\text { Gesellschaft }\end{array}$ & $4,37(0,96)$ & $4,54(0,69)$ & $-0,932$ & $-0,555$ \\
\hline $\begin{array}{l}\text { Der Wille, an der Gesellschaft } \\
\text { teilzunehmen }\end{array}$ & $4,43(0,77)$ & $4,52(0,72)$ & $-0,507$ &,- 456 \\
\hline $\begin{array}{l}\text { Kontakte zwischen Menschen } \\
\text { verschiedener Kulturen }\end{array}$ & $4,03(0,99)$ & $4,50(0,84)$ & $-2,200 *$ & $-2,344 *$ \\
\hline $\begin{array}{l}\text { Mitgliedschaft/ } \\
\text { Engagement in Organisationen }\end{array}$ & $3,40(1,00)$ & $3,36(1,07)$ & 0,181 &,- 227 \\
\hline Anerkennung durch Andere & $3,77(0,94)$ & $4,09(0,82)$ & $-1,575$ & $-1,386$ \\
\hline $\begin{array}{l}\text { Vorhandensein eines } \\
\text { Zugehörigkeitsgefühls }\end{array}$ & $3,97(0,73)$ & $4,46(0,59)$ & $-3,210 * *$ & $-2,929 * *$ \\
\hline Gleiche Rechte für Alle & $4,70(0,53)$ & $4,78(0,51)$ & $-0,675$ & $-0,899$ \\
\hline Bildungsstand & $2,90(1,18)$ & $3,17(1,14)$ & $-1,008$ & $-0,936$ \\
\hline Berufliche Stellung & $2,50(1,07)$ & $2,56(1,07)$ & $-0,260$ & $-0,374$ \\
\hline Zugang zum Wohnungsmarkt & $3,60(1,25)$ & $3,83(1,12)$ & $-0,821$ & $-0,711$ \\
\hline $\begin{array}{l}\text { Zufriedenheit mit beruflicher und } \\
\text { sozialer Situation }\end{array}$ & $3,83(0,91)$ & $4,13(0,81)$ & $-1,491$ & $-1,467$ \\
\hline $\begin{array}{l}\text { Wenn Zuwanderer ihre Kultur } \\
\text { beibehalten möchten, sollten sie unter } \\
\text { sich bleiben }\end{array}$ & $1,83(0,83)$ & $1,70(0,94)$ & 0,652 & $-1,024$ \\
\hline $\begin{array}{l}\text { Es wäre gut, wenn alle Zuwanderer } \\
\text { in Deutschland ihre Kulturen } \\
\text { beibehielten }\end{array}$ & $2,97(0,81)$ & $3,04(1,01)$ & $-0,350$ & $-0,155$ \\
\hline $\begin{array}{l}\text { Menschen, die nach Deutschland } \\
\text { kommen, sollten ihr Verhalten der } \\
\text { deutschen Kultur anpassen }\end{array}$ & $3,57(0,82)$ & $3,26(0,99)$ & 1,439 & $-1,371$ \\
\hline $\begin{array}{l}\text { Zuwanderer sollten sowohl in ihre } \\
\text { Herkunftsgesellschaft als auch in die } \\
\text { deutsche Gesellschaft eingebunden } \\
\text { sein }\end{array}$ & $4,27(0,78)$ & $4,32(0,94)$ & $-0,255$ & $-0,631$ \\
\hline
\end{tabular}

Quelle: Eigene Darstellung; Anmerkungen: Standardabweichung in Klammern, V= Ungleiche Varianzen, ${ }^{*} \mathrm{p}<$ 0,$05 ; * * p<0,01$.

Bezüglich der Items zu Kontakten zwischen Personen verschiedener Kulturen und dem Vorhandensein eines Zugehörigkeitsgefühls zeigt sich in beiden Fällen ein höherer Mittelwert bei Migrantinnen und Migranten zweiter Generation. Dabei weist das Item Kontakte zwischen Personen verschiedener Kulturen eine Mittelwertdifferenz von 0,47 und das Item 
Vorhandensein eines Zugehörigkeitsgefühls eine Differenz von 0,47 auf. Diese Differenzen fallen deutlich höher aus als bei den Vergleichen zwischen Personen mit und ohne Migrationshintergrund.

Die Verteilung des Items Kontakte zwischen Personen verschiedener Kulturen zeigt, dass der Großteil der Migrantinnen und Migranten zweiter Generation diese als sehr wichtig für Integration einschätzt (67,4 \%) (vgl. Abbildung 14). Bei Personen der ersten Generation wird dieses Item zwar auch von den meisten Befragten als sehr wichtig eingeschätzt (40,0\%), jedoch geben in dieser Gruppe auch 26,7 \% „Teils, teils“ an und nehmen damit eine unentschlossene Position bezüglich dieses Items ein.

Abbildung 14: Verteilung des Items Kontakte zwischen Menschen verschiedener Kulturen in Prozent nach Migrantinnen und Migranten erster und zweiter Generation

Kontakte zwischen Menschen verschiedener Kulturen

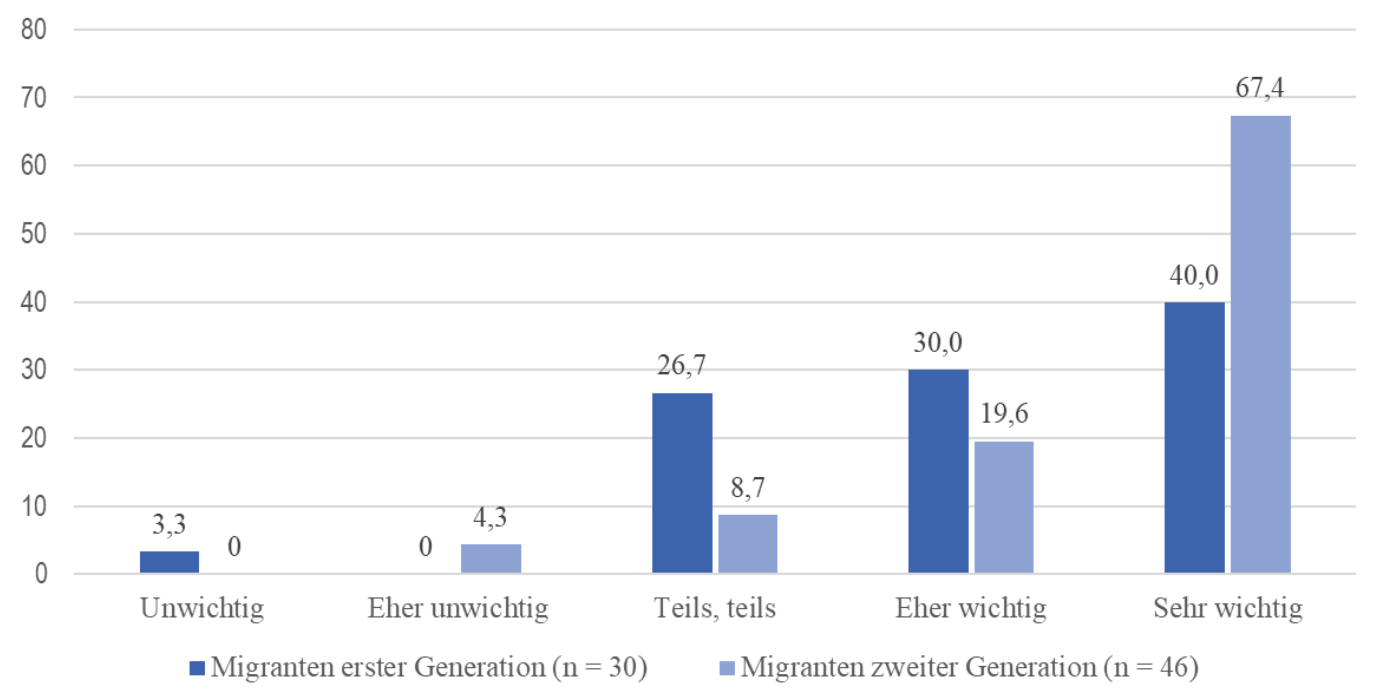

Quelle: Eigene Darstellung.

Beim Item Vorhandensein eines Zugehörigkeitsgefühls, dessen Verteilung in Abbildung 15 dargestellt ist, zeigt sich ebenfalls eine deutlich höhere Besetzung der Mittelkategorie bei Migrantinnen und Migranten erster Generation (17,2 \%). Zudem bewerten nur 20,7 \% der Migrantinnen und Migranten erster Generation das Vorhandensein des Zugehörigkeitsgefühls als sehr wichtig für Integration, wohingegen 50,0 \% der Migrantinnen und Migranten zweiter Generation diesen Aspekt als sehr wichtig einschätzen. 
Abbildung 15: Verteilung des Items Vorhandensein eines Zugehörigkeitsgefühls in Prozent nach Migrantinnen und Migranten erster und zweiter Generation

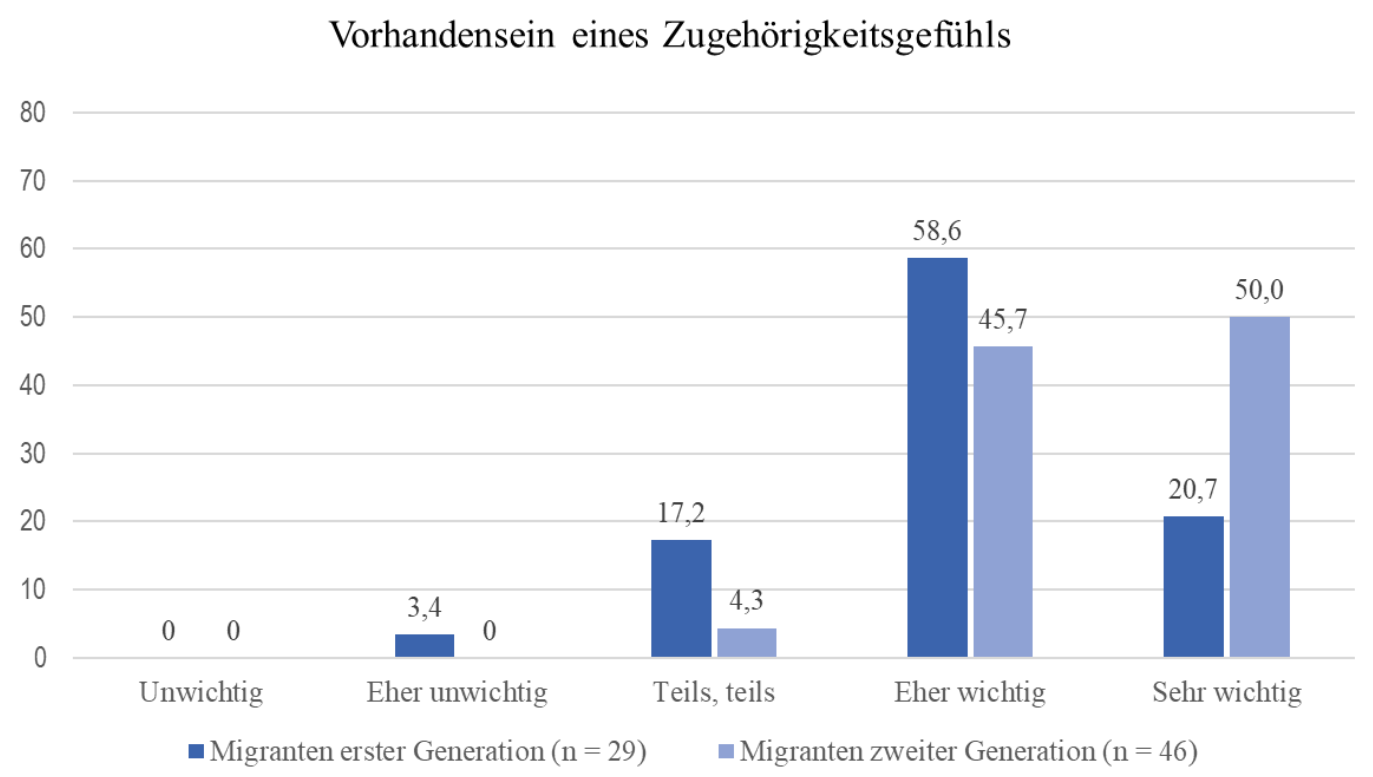

Quelle: Eigene Darstellung.

Ein weiterer vorgenommener Vergleich zwischen Personen mit europäischem und außereuropäischem Migrationshintergrund zeigt bezüglich dieser Gruppen signifikante Unterschiede in der Bewertung der Aspekte gute Deutschkenntnisse und Kontakte zwischen Menschen verschiedener Kulturen. Beide Items werden von Personen mit einem europäischen Migrationshintergrund als wichtiger für Integration eingeschätzt ${ }^{25}$. Aufgrund der geringen Gruppengrößen konnte bei diesem Vergleich keine Trennung nach Generationen vorgenommen werden. Die Analysen beziehen sich somit auf Personen mit und ohne eigene Migrationserfahrung, die anhand des eigenen Geburtslandes oder des Geburtslandes der Eltern einem europäischen oder außereuropäischen Hintergrund zugeordnet wurden.

\subsubsection{Zusammenhänge zwischen Ergebnissen und Aspekten von Integration}

Innerhalb dieses Unterkapitels werden die Zusammenhänge zwischen Ergebnissen und Aspekten von Integration dargestellt. Dazu erfolgt zunächst die Darstellung der Zusammenhänge unter den Items zu Ergebnissen von Integration, bevor auf die Zusammenhänge zwischen Ergebnissen und Aspekten eingegangen wird ${ }^{26}$.

\footnotetext{
25 Siehe Anhang

${ }^{26}$ Die Aspekte von Integration weisen auch untereinander vielfältige Zusammenhänge auf, die im Rahmen der Arbeit jedoch nicht weiter ausgeführt werden sollen. Die Ergebnisse dieser Analysen sind im Anhang zu finden.
} 
Tabelle 25 zeigt die Zusammenhänge zwischen den abgefragten Ergebnissen von Integration anhand von Spearman's Rho.

In Bezug auf den Ausgang zur Segregation (,Wenn Zuwanderer ihre Kultur beibehalten möchten, sollten sie unter sich bleiben“") zeigen sich signifikante negative Zusammenhänge mit den Items zu Pluralismus und Mehrfachintegration. Mit dem Item zur Assimilation besteht hingegen ein signifikanter positiver Zusammenhang. Demnach geht die Befürwortung der Segregation auch mit der Forderung der Anpassung einher, wohingegen die Beibehaltung der Herkunftskulturen sowie die Mehrfachintegration eher abgelehnt werden.

Das Item zu Pluralismus („,Es wäre gut, wenn alle Zuwanderer in Deutschland ihre Kulturen beibehielten“) weist neben dem negativen Zusammenhang mit dem Ausgang der Segregation auch einen signifikanten negativen Zusammenhang mit dem Ausgang der Assimilation auf. In Bezug auf die Mehrfachintegration zeigt sich hingegen ein signifikanter positiver Zusammenhang. Mit einer steigenden Zustimmung zum Konzept des Pluralismus werden die Konzepte Segregation und Assimilation somit eher abgelehnt, während die Befürwortung einer Mehrfachintegration steigt. Sowohl das Item zur Assimilation als auch das zur Mehrfachintegration weisen lediglich signifikante Zusammenhänge mit den Items zu Segregation und Pluralismus auf. Zwischen den beiden Items besteht hingegen kein signifikanter Zusammenhang. Insgesamt muss jedoch festgehalten werden, dass alle dargestellten signifikanten Zusammenhänge schwach ausfallen.

Tabelle 25: Zusammenhänge zwischen Ergebnissen von Integration anhand von Spearman's rho

\begin{tabular}{|c|c|c|c|c|}
\hline Item & Segregation $^{27}$ & Pluralismus & Assimilation & $\begin{array}{l}\text { Mehrfach- } \\
\text { integration }\end{array}$ \\
\hline $\begin{array}{l}\text { Wenn Zuwanderer ihre } \\
\text { Kultur beibehalten möchten, } \\
\text { sollten sie unter sich bleiben } \\
\text { (Segregation) }\end{array}$ & 1,000 & $-0,126 *$ & $0,172 * *$ & $-0,186^{* *}$ \\
\hline $\begin{array}{l}\text { Es wäre gut, wenn alle } \\
\text { Zuwanderer in Deutschland } \\
\text { ihre Kulturen beibehielten } \\
\text { (Pluralismus) }\end{array}$ & $-0,126 *$ & 1,000 & $-0,118 *$ & $0,227 * *$ \\
\hline $\begin{array}{l}\text { Menschen, die nach } \\
\text { Deutschland kommen, } \\
\text { sollten ihr Verhalten der } \\
\text { deutschen Kultur anpassen } \\
\text { (Assimilation) }\end{array}$ & $0,172 * *$ & $-0,118^{*}$ & 1,000 & $-0,090$ \\
\hline
\end{tabular}

\footnotetext{
${ }^{27}$ Aus Platzgründen werden die Ergebnisse von Integration hier nicht anhand der Formulierung aus dem Fragebogen dargestellt.
} 


\begin{tabular}{l|l|l|l|l}
\hline $\begin{array}{l}\text { Zuwanderer sollten sowohl } \\
\text { in ihre Herkunftsgesellschaft } \\
\text { als auch in die deutsche }\end{array}$ & $\mathbf{- 0 , 1 8 6 * *}$ & $\mathbf{0 , 2 2 7 * *}$ & $-0,090$ & 1,000 \\
$\begin{array}{l}\text { Gesellschaft eingebunden } \\
\text { sein (Mehrfachintegration) }\end{array}$ & & & & \\
\hline
\end{tabular}

Quelle: Eigene Darstellung, ${ }^{*} \mathrm{p}<0,05 ; * * \mathrm{p}<0,01$.

Auch in Bezug auf die Aspekte von Integration zeigen sich einige signifikante Zusammenhänge mit den Ergebnissen von Integration. So zeigen sich beim Item zur Segregation signifikante positive Zusammenhänge mit den Items zur Anerkennung von Werten, dem Bildungsstand und der beruflichen Stellung. Obwohl die Zugewanderten laut diesem Item unter sich bleiben sollen geht eine Befürwortung der Segregation gleichzeitig mit einer höheren Bedeutung dieser Aspekte einher. Zugewanderte sollten somit wichtige Werte anerkennen sowie über einen gewissen Bildungsstand und berufliche Stellung verfügen. Gleichzeitig zeigen sich bei diesem Item signifikante negative Zusammenhänge mit den Aspekten zu Chancen zur Teilnahme an der Gesellschaft, Kontakten zwischen Menschen verschiedener Kulturen, gleichen Rechten für alle und Zugang zum Wohnungsmarkt. Dies lässt vermuten, dass eine Befürwortung der Segregation insgesamt auch mit einem Ausschluss von Zugewanderten aus der Gesellschaft einhergeht bzw. damit, dass keine Einbindung stattfinden soll. Das Item zum Pluralismus weist hingegen einen signifikanten negativen Zusammenhang mit der Anerkennung wichtiger Werte sowie signifikante positive Zusammenhänge mit den Items zu Kontakten zwischen Menschen verschiedener Kulturen, dem Vorhandensein eines Zugehörigkeitsgefühls, gleichen Rechten für alle und dem Zugang zum Wohnungsmarkt auf. Die Befürwortung des Pluralismus geht somit mit einer geringeren Bedeutung der Anerkennung wichtiger Werte einher, während die Interaktion zwischen Menschen unterschiedlicher Kulturen, ein Zugehörigkeitsgefühl sowie eine Gleichbehandlung in Bezug auf Rechte und den Zugang zum Wohnungsmarkt eine bedeutendere Rolle spielen. Die Kontakte zwischen Menschen unterschiedlicher Kulturen, das Zugehörigkeitsgefühl sowie die rechtliche Gleichstellung weisen auch in Bezug auf die Mehrfachintegration signifikante positive Zusammenhänge auf. Hier zeigt sich jedoch zusätzlich ein signifikanter positiver Zusammenhang mit dem Willen an der Gesellschaft teilzunehmen. In Bezug auf die Assimilation zeigen sich bei allen Items zu Kulturation sowie bei denen zur Interaktion (außer Mitgliedschaft/Engagement in Organisationen) signifikante Zusammenhänge. Im Bereich der Kulturation (Werte und Sprache) fallen diese positiv aus. Ein positiver signifikanter Zusammenhang zeigt sich beim Ausgang der Assimilation auch bei den Aspekten Wille an der Gesellschaft teilzunehmen und Bildungsstand. Negative signifikante Zusammenhänge zeigen sich hingegen bei den Aspekten Chancen zur Teilnahme an der Gesellschaft und Kontakte zwischen Menschen verschiedener Kulturen. Die Befürwortung der 
Assimilation scheint somit, ähnlich wie bei der Segregation, mit einer Ablehnung der Einbindung von Zugewanderten einherzugehen. Gleichzeitig sollen Zugewanderte jedoch Werte anerkennen und gute Deutschkenntnisse aufweisen.

Tabelle 26: Zusammenhänge zwischen Ergebnissen und Aspekten von Integration anhand von Spearman's rho

\begin{tabular}{|c|c|c|c|c|}
\hline Item & Segregation $^{28}$ & Pluralismus & Assimilation & $\begin{array}{l}\text { Mehrfach- } \\
\text { integration }\end{array}$ \\
\hline Gute Deutschkenntnisse & 0,049 & $-0,094$ & $0,282 * *$ & $-0,074$ \\
\hline $\begin{array}{l}\text { Anerkennung wichtiger } \\
\text { Werte }\end{array}$ & $0,123 *$ & $-0,100 *$ & $0,302 * *$ & 0,022 \\
\hline $\begin{array}{l}\text { Chancen zur Teilnahme an } \\
\text { der Gesellschaft }\end{array}$ & $-0,116 *$ & 0,074 & $-0,102 *$ & 0,084 \\
\hline $\begin{array}{l}\text { Der Wille, an der } \\
\text { Gesellschaft teilzunehmen }\end{array}$ & $-0,027$ & $-0,007$ & $0,200 * *$ & $0,146 * *$ \\
\hline $\begin{array}{l}\text { Kontakte zwischen } \\
\text { Menschen verschiedener } \\
\text { Kulturen (bspw. } \\
\text { Freundschaften, } \\
\text { Partnerschaften) }\end{array}$ & $-0,206 * *$ & $0,169 * *$ & $-0,112 *$ & $0,189 * *$ \\
\hline $\begin{array}{l}\text { Mitgliedschaft/Engagement } \\
\text { in Organisationen }\end{array}$ & 0,047 & 0,020 & 0,013 & 0,027 \\
\hline Anerkennung durch andere & $-0,061$ & 0,038 & $-0,075$ & 0,045 \\
\hline $\begin{array}{l}\text { Vorhandensein eines } \\
\text { Zugehörigkeitsgefühls }\end{array}$ & $-0,090$ & $0,111 *$ & $-0,032$ & $0,216 * *$ \\
\hline Gleiche Rechte für alle & $-0,134 * *$ & $0,160 * *$ & $-0,061$ & $0,209 * *$ \\
\hline Bildungsstand & $0,127 *$ & 0,027 & $0,146 * *$ & $-0,071$ \\
\hline Berufliche Stellung & $0,198 * *$ & $-0,013$ & 0,093 & $-0,072$ \\
\hline $\begin{array}{l}\text { Zugang zum } \\
\text { Wohnungsmarkt }\end{array}$ & $-0,105 *$ & $0,119 *$ & $-0,085$ & 0,057 \\
\hline $\begin{array}{l}\text { Zufriedenheit mit } \\
\text { beruflicher und sozialer } \\
\text { Situation }\end{array}$ & $-0,049$ & 0,066 & $-0,011$ & 0,086 \\
\hline
\end{tabular}

Quelle: Eigene Darstellung, ${ }^{*} \mathrm{p}<0,05 ; * * \mathrm{p}<0,01$.

\footnotetext{
${ }^{28}$ Aus Platzgründen werden die Ergebnisse von Integration hier nicht anhand der Formulierung aus dem Fragebogen dargestellt.
} 
6.1.3 Zusammenhänge zwischen der Zustimmung zu Integrationskonzepten und sozialstrukturellen Merkmalen der Befragten

Im Rahmen der Analyse der Zusammenhänge zwischen der Zustimmung zu Integrationskonzepten und sozialstrukturellen Merkmalen sowie Merkmalen der Sportvereine sollen die folgenden Hypothesen untersucht werden:

- H1: Das Alter der Befragten weist einen negativen Zusammenhang mit der Unterstützung pluralistischer Konzepte auf.

- H2: Personen mit Migrationshintergrund unterstützen pluralistische Konzepte stärker als andere Konzepte.

- H3: Umso höher der Anteil von Personen mit Migrationshintergrund in der Trainingsgruppe, desto stärker werden pluralistische Konzepte unterstützt.

- H4: Umso höher der Bildungsstand, desto stärker werden pluralistische Konzepte unterstützt.

Tabelle 25 zeigt die Zusammenhänge zwischen den Einstellungen zu verschiedenen Ausgängen von Integration und Merkmalen der Individuen sowie der Trainingsgruppe anhand von Spearman's Rho.

Tabelle 27: Zusammenhänge zwischen sozialstrukturellen Merkmalen sowie Merkmalen der Sportvereine und der Zustimmung zu Integrationskonzepten anhand von Spearman's rho

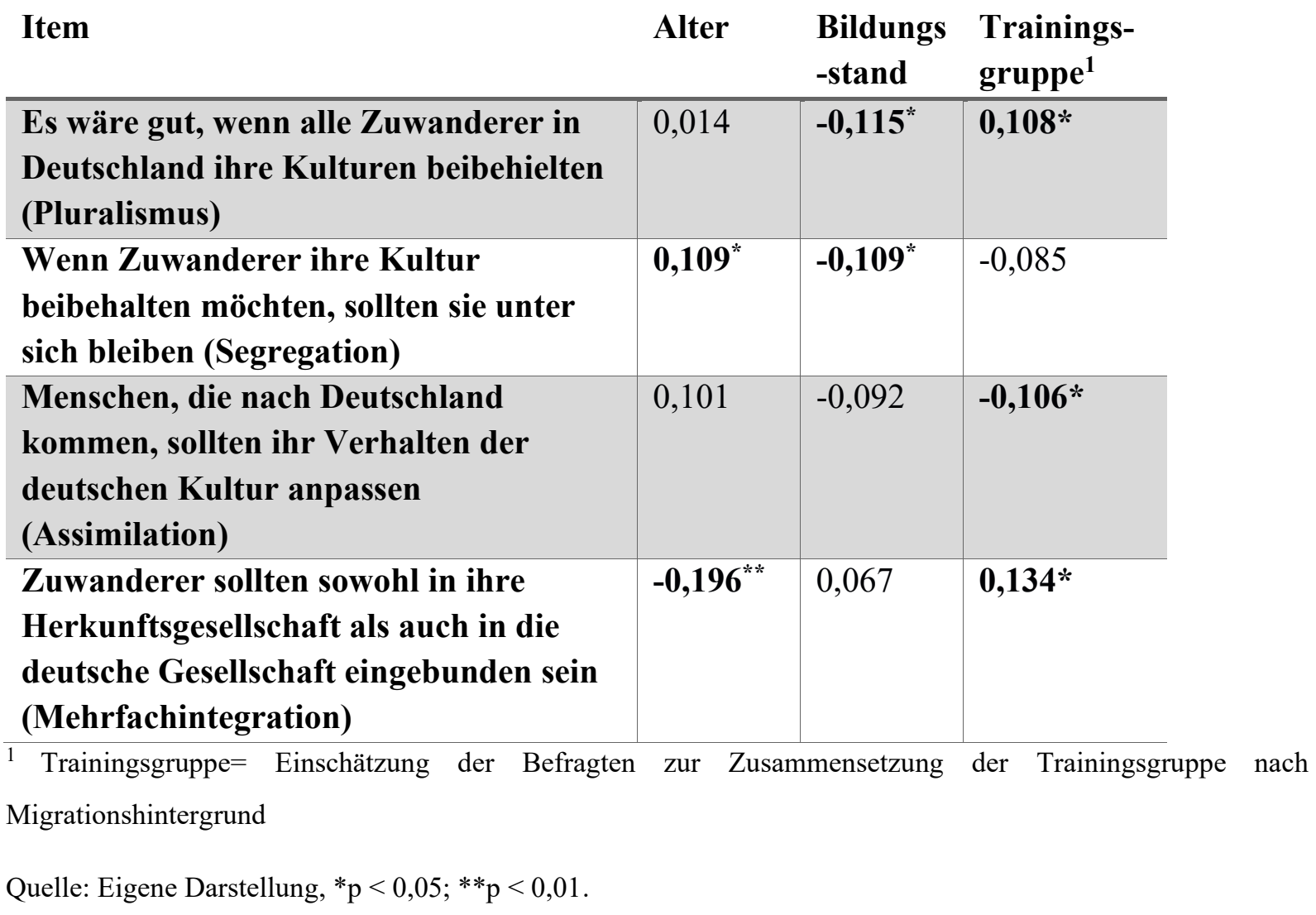


In Bezug auf das Alter zeigt sich ein signifikanter positiver Zusammenhang mit dem Konzept der Segregation als auch ein signifikanter negativer Zusammenhang mit der Unterstützung der Mehrfachintegration. Umso höher das Alter, desto weniger wird somit das Konzept der Mehrfachintegration unterstützt, während die Zustimmung zur Segregation zunimmt. Mit steigendem Alter wird somit zunehmend unterstützt, dass Zugewanderte, die ihre Kultur beibehalten wollen, unter sich bleiben sollen. Demnach kann H1, wonach ein negativer Zusammenhang zwischen dem Alter der Befragten und der Unterstützung pluralistischer Konzepte angenommen wird, nicht bestätigt werden, da sich beim Item „Es wäre gut, wenn alle Zuwanderer in Deutschland ihre Kulturen beibehielten“ kein signifikanter Zusammenhang zeigt. Dennoch kann festgehalten werden, dass die Zustimmung zur Mehrfachintegration mit steigendem Alter sinkt, während die Befürwortung der Segregation steigt. Dies zeigt, dass mit steigendem Alter eher eine Abgrenzung zwischen einheimischen Personen und Zugewanderten, die ihre Kultur beibehalten wollen, favorisiert wird.

Beim Bildungsstand zeigt sich ein signifikanter negativer Zusammenhang mit der Aussage zum Konzept des Pluralismus unter Beibehaltung der Kulturen. Umso höher der Bildungsstand, desto weniger wird ein pluralistisches Konzept, in dem alle Zugewanderten ihre Kulturen beibehalten, demnach unterstützt. Dies widerspricht Hypothese H4, innerhalb derer von einer stärkeren Unterstützung pluralistischer Konzepte mit steigendem Bildungsstand ausgegangen wird. In Bezug auf das Item zur Segregation zeigt sich darüber hinaus ein signifikanter negativer Zusammenhang mit dem Bildungsstand. Somit besteht zwar ein negativer Zusammenhang mit pluralistischen Ansichten, die die Beibehaltung aller Kulturen befürwortet, entscheiden sich Zugewanderte jedoch für die Beibehaltung ihrer Kultur, sollte es auch nicht zur Segregation kommen.

Die Zusammensetzung der Trainingsgruppe weist einen signifikanten positiven Effekt mit der Aussage zum Pluralismus sowie einen signifikanten negativen Zusammenhang mit dem assimilativen Konzept und einen signifikanten positiven Zusammenhang mit dem Konzept der Mehrfachintegration auf. Umso höher der Anteil von Personen mit Migrationshintergrund in der Trainingsgruppe ist, desto höher ist somit die Zustimmung zum pluralistischen Item. Darüber hinaus führt ein steigender Anteil von Personen mit Migrationshintergrund in der Trainingsgruppe zu einer abnehmenden Unterstützung des assimilativen Konzepts und auch zu einer steigenden Unterstützung des Konzepts der Mehrfachintegration. Demnach wird mit einem steigenden Anteil von Personen mit Migrationshintergrund in der Trainingsgruppe sowohl eine Beibehaltung der Kulturen, als auch der Einbezug in die deutsche Gesellschaft und 
Herkunftsgesellschaft zunehmend unterstützt. Dies bestätigt die zu untersuchende Hypothese H3, in der angenommen wurde, dass mit einem höheren Anteil von Personen mit Migrationshintergrund in der Trainingsgruppe die Unterstützung pluralistischer Konzepte steigt.

Der Zusammenhang zwischen der Zustimmung $\mathrm{zu}$ Integrationskonzepten und dem Migrationshintergrund wird anhand des Eta-Koeffizienten untersucht, um eine Aussage zur Hypothese H2 treffen zu können (vgl. Tabelle 26). Der Eta-Koeffizient zeigt, zu welchem Teil die Varianz, der abhängigen metrischen Variable, durch die nominale Variable erklärt werden kann. Hier zeigt sich lediglich beim Pluralismus-Item ein signifikanter Effekt. Dieser besitzt jedoch eine geringe Effektstärke. Zusätzlich zeigt Eta-Quadrat, dass sich lediglich 1,6 \% der Varianz der abhängigen Variable durch den Migrationshintergrund erklären lassen. Dennoch lässt sich Hypothese H2, die von einem Zusammenhang zwischen Migrationshintergrund und der Unterstützung pluralistischer Konzepte ausgeht, damit bestätigen.

Tabelle 28: Zusammenhänge zwischen Migrationshintergrund und der Zustimmung zu Integrationskonzepten anhand von Eta

\begin{tabular}{l|l|l}
\hline Item & Eta-Koeffizient & Eta $^{\mathbf{2}}$ \\
\hline $\begin{array}{l}\text { Es wäre gut, wenn alle Zuwanderer in } \\
\text { Deutschland ihre Kulturen beibehielten } \\
\text { (Pluralismus) }\end{array}$ & $\mathbf{0 , 1 2 6 *}$ & 0,016 \\
\hline $\begin{array}{l}\text { Wenn Zuwanderer ihre Kultur beibehalten } \\
\text { möchten, sollten sie unter sich bleiben } \\
\text { (Segregation) }\end{array}$ & 0,071 & 0,005 \\
\hline $\begin{array}{l}\text { Menschen, die nach Deutschland kommen, } \\
\text { sollten ihr Verhalten der deutschen Kultur } \\
\text { anpassen (Assimilation) }\end{array}$ & 0,025 & 0,001 \\
\hline $\begin{array}{l}\text { Zuwanderer sollten sowohl in ihre } \\
\text { Herkunftsgesellschaft als auch in die deutsche } \\
\text { Gesellschaft eingebunden sein } \\
\text { (Mehrfachintegration) }\end{array}$ & 0,037 & 0,001 \\
\hline
\end{tabular}

Quelle: Eigene Darstellung; Anmerkung: Signifikanz anhand einer univariaten Varianzanalyse. 


\subsubsection{Zusammenfassung und Diskussion der quantitativen Ergebnisse}

Insgesamt zeigt sich, dass die Befragten die einzelnen Aspekte in Bezug auf ihre Bedeutung für Integration überwiegend positiv bewerten. Gleichzeitig zeigt sich auch ein vermehrter Zuspruch für pluralistische Ausgänge von Integration. Diese Ergebnisse könnten aber auch auf die Zusammensetzung der Stichprobe zurückzuführen sein, die überwiegend Personen mit hohem Bildungsstand enthält. Jedoch zeigen Studien, dass die Mitgliedschaft in Sportvereinen einer gewissen Selektivität unterliegt und die Mitgliedschaftsquote mit höherer Bildung zunimmt (Mutz/Burrmann 2015: 86). Somit kann davon ausgegangen werden, dass es sich um eine spezifische Zusammensetzung der Sportvereine handelt und die Ergebnisse so die Sichtweisen in Sportorganisationen gut abbilden können.

Von besonderer Bedeutung im Rahmen der Aspekte von Integration sind für die Befragten die rechtliche Gleichstellung, der Wille an der Gesellschaft teilzunehmen und Chancen zur Teilnahme an der Gesellschaft. Gerade die beiden Aspekte Chancen und Wille zur Teilnahme an der Gesellschaft kommen in den klassischen Integrationskonzepten nicht vor. Diese werden lediglich im Bielefelder Desintegrationsansatz nach Anhut und Heitmeyer (2009) aufgeführt, welcher kein klassisches Integrationskonzept darstellt, sondern der Erklärung von Phänomenen wie Gewalt(-kriminalität), Rechtsextremismus, ethnisch-kulturellen Konflikten sowie der Abwertung und Abwehr schwacher Gruppen dient. Dennoch scheinen die dort aufgeworfenen Aspekte, aus Sicht der Befragten, die größte Bedeutung für Integration innerhalb der Dimension der Interaktion zu haben. Gleichzeitig sind die meisten hoch bewerteten Aspekte der Dimension der Interaktion zuzurechnen. Eine Differenzierung der Bedeutung der Aspekte hinsichtlich der Akteursebenen lässt sich nicht ausmachen.

Ausnahmen in der überwiegend positiven Bewertung der Aspekte bilden lediglich der Bildungsstand und die berufliche Stellung, welche von den Befragten eher als weniger wichtig eingeschätzt werden. Dies könnte, zumindest bei der beruflichen Stellung, darauf zurückzuführen sein, dass es sich dabei um einen Aspekt handelt, der eine gewisse Relativität aufweist. Solche Aspekte als Maßstab für Integration zu verwenden, wurden auch in der Studie von Schramkowksi im Rahmen der Expertinnen- und Experteninterviews kritisch gesehen, da diese Integration als einen nicht konstanten Zustand darstellen, der im Falle von beispielsweise plötzlicher Arbeitslosigkeit nicht mehr erfüllt wäre (Schramkowski 2007: 148-149). Es zeigt sich weiter, dass die Aspekte der Platzierung, und somit diese Dimension als weniger bedeutend bewertet werden als die Dimension der Interaktion. Dies geht mit den Ergebnissen zu Perspektiven auf Integration von Schülerinnen und Schülern von Lutter (2011) einher. 
Innerhalb dieser Studie wurden von den Schülerinnen und Schülern überwiegend Aspekte einer sozial-kulturellen Perspektive angesprochen, wohingegen Aspekte der strukturellen Integration nicht thematisiert wurden.

Außerdem wird dem Aspekt der Sprache, mit einem Mittelwert von 3,87, eine geringere Bedeutung zugemessen und dieser Aspekt erscheint erst in der zweiten Hälfte der nach ihrer Bedeutung sortierten Items. Dies ist überraschend, da gerade dieser Aspekt in der Literatur und auch in politischen Programmen immer wieder als sehr bedeutend für Integration herausgestellt wird. Die jungen Erwachsenen mit Migrationshintergrund führten in der Studie von Schramkowksi (2009) aus, Integration würde von Personen ohne Migrationshintergrund alleinig mit dem Erwerb der Sprache gleichgesetzt werden. Dies lässt sich anhand der vorliegenden Daten nicht bestätigen. Vielmehr werden Aspekte der Interaktion als bedeutender für Integration angesehen.

Betrachtet man die Bewertung der Items zu Ausgängen von Integration, zeigt sich, dass vor allem der Ausgang der Mehrfachintegration favorisiert wird. Dennoch zeigen sich gleichzeitig auch assimilative Tendenzen, so dass hier eine Mischung zwischen diesen beiden Konzepten angenommen werden kann. Die größte Ablehnung zeigt sich hingegen beim Ausgang der Segregation. Dies geht mit den Ergebnissen von Strahle und Baur (2009) sowie Baur und Mutz (2009) einher, in deren qualitativen und quantitativen Untersuchungen ebenfalls eine Mischung von assimilativen und pluralistischen Tendenzen festgestellt wurde.

Beim Vergleich der Bewertungen durch Vereinsmitglieder und Vorstände zeigt sich bei den Aspekten von Integration lediglich ein signifikanter Unterschied beim Item zu Mitgliedschaft/Engagement in Organisationen. In Bezug auf die Ausgänge von Integration zeigen sich hingegen keine signifikanten Unterschiede.

Im Gegensatz dazu zeigen sich beim Vergleich von Personen mit und ohne Migrationshintergrund mehrere signifikante Unterschiede in der Bewertung von Aspekten und Ausgängen von Integration. Sowohl die Aspekte der Chancen zur Teilnahme an der Gesellschaft, die rechtliche Gleichstellung und Kontakte zwischen Menschen unterschiedlicher Kulturen, als auch der Ausgang mit Bezug zum Konzept des Pluralismus unter Beibehaltung der Kulturen, weisen in diesem Vergleich signifikante Unterschiede auf. Dabei werden diese Items von den Personen mit Migrationshintergrund höher bewertet. Diese unterschiedliche Bewertung könnte auf Erfahrungen der Personen mit Migrationshintergrund beruhen, die zu einer höheren Sensibilität in manchen Bereichen der Integration führen. So könnten Personen mit Migrationshintergrund beispielsweise in Bezug auf die Möglichkeiten der Teilnahme und 
rechtlichen Gleichstellung Erfahrungen gemacht haben, mit denen Personen ohne Migrationshintergrund nicht konfrontiert werden. Dadurch könnten Personen mit Migrationshintergrund vorliegende Differenzen bezüglich der Partizipationsmöglichkeiten und rechtlichen Gleichstellung eher bewusst sein, während Personen ohne Migrationshintergrund mit solchen Problematiken nicht konfrontiert werden. Die als höher angesehene Bedeutung von Kontakten zwischen Menschen unterschiedlicher Kulturen könnte aus einer größeren Offenheit für andere Kulturen resultieren, da für Personen mit Migrationshintergrund auch immer eine Akzeptanz ihrer Kultur durch Andere eine Rolle spielt. Dafür spricht auch der signifikant höhere Zuspruch zum Item „Es wäre gut, wenn alle Zuwanderer in Deutschland ihre Kulturen beibehielten“. Gleichzeitig könnten Personen mit Migrationshintergrund auch generell häufiger über interethnische Kontakte verfügen als Personen ohne Migrationshintergrund, wodurch diese auch als wichtiger eingeschätzt werden. Dies beschreibt beispielsweise Haug (2006) anhand einer Analyse des Integrationssurveys des Bundesinstituts für Bevölkerungsforschung am Beispiel italienischer und türkischer Migrantinnen und Migranten. Dabei zeigte sich, dass interethnische Kontakte zu Deutschen und Personen aus anderen Ländern bei Migrantinnen und Migranten häufig vorkommen, diese interethnischen Kontakte bei Deutschen aber eher eine Ausnahme sind (Haug 2006: 77/78). Zudem stellt Esser (2006) heraus, dass Freundschaften mit Personen des Aufnahmelandes für Zugewanderte und ihre Nachkommen von Vorteil sein können, da diese Zugänge zu hilfreichen Ressourcen im Aufnahmeland öffnen.

Zudem zeigte sich in den Ergebnissen, dass der Aspekt Kontakte zu Menschen verschiedener Kulturen auch von Migrantinnen und Migranten zweiter Generation als bedeutender für Integration eingeschätzt wird als von Migrantinnen und Migranten erster Generation. Gleiches zeigt sich auch beim Vergleich von Personen mit europäischem und außereuropäischem Migrationshintergrund. Dabei schätzen Personen mit europäischem Migrationshintergrund diesen Aspekt als wichtiger ein. Auch beim Vergleich der Generationen lässt sich die höhere zugemessene Bedeutung mit vermehrten interethnischen Kontakten der zweiten Generation begründen. Eine Zunahme interethnischer Freundschaften im Generationenverlauf zeigt sich beispielsweise bei Schacht, Kristen und Tucci (2014). Darüber hinaus wurden in der Arbeit Unterschiede zwischen unterschiedlichen Gruppen von Migrantinnen und Migranten festgestellt. So zeigte sich, dass türkischstämmige Befragte, als einzige untersuchte außereuropäische Gruppe, weniger Freundschaften zu Deutschen aufwiesen als alle anderen untersuchten europäischen Gruppen (Schacht/Kristen/Tucci 2014: 456). Somit könnte auch bei der unterschiedlichen Bewertung des Aspekts durch Personen mit europäischem und außereuropäischem Migrationshintergrund vermehrt vorhandene interethnische Kontakte auf 
Seiten der Personen mit europäischem Migrationshintergrund eine Rolle spielen. Zudem wird im Rahmen der Arbeit ausgeführt, dass diese Unterschiede vor allem in Verbindung mit vorhandenen Deutschkenntnissen stehen. Diese werden nämlich zunächst benötigt, um mit der Bevölkerung des Aufnahmelandes zu kommunizieren, gleichzeitig können diese aber auch kulturelle Distanzen vor dem Hintergrund einer gemeinsamen Sprache abmildern (Schacht/Kristen/Tucci 2014: 456/457). Dieser Zusammenhang zwischen vorhandenen interethnischen Kontakten und Sprachkenntnissen könnte somit auch erklären, wieso Personen mit europäischem Migrationshintergrund dem Aspekt gute Deutschkenntnisse eine höhere Bedeutung zumessen als Personen mit außereuropäischem Migrationshintergrund.

Insgesamt scheinen die Gruppenunterschiede in Bezug auf die Bedeutung der Kontakte zwischen Menschen verschiedener Kulturen somit überwiegend durch vermehrte interethnische Kontakte der Gruppen, die diesen Aspekt als bedeutender einschätzen, gekennzeichnet zu sein. Abbildung 16 stellt die Ergebnisse der Analysen zusammenfassend dar. Dabei sind sowohl Dimensionen nach ihrem Gesamtmittelwert in Bezug auf ihre Bedeutung geordnet als auch die einzelnen Aspekte innerhalb der Dimensionen. 
Abbildung 16: Bedeutung der unterschiedlichen Dimensionen und Aspekte von Integration nach Ansicht der Befragten

\section{Interaktion}

1. Wille an der Gesellschaft teilzunehmen, 2. Chancen zur Teilnahme an der Gesellschaft,

3. Interethnische Kontakte, 4. Anerkennung durch Andere, 5. Mitgliedschaft/Engagement

\section{Identifikation}

Zugehörigkeitsgefühl

\section{Kulturation}

1. Anerkennung wichtiger Werte, 2. Gute Deutschkenntnisse

\section{Platzierung}

1. Gleiche Rechte für Alle, 2. Zufriedenheit mit beruflicher und sozialer Stellung, 3.

Zugang zum Wohnungsmarkt, 4. Bildungsstand, 5. Berufliche Stellung

Quelle: Eigene Darstellung; Farblich hervorgehoben sind die Aspekte, bei denen sich signifikante Unterschiede zwischen Personen mit und ohne Migrationshintergrund gezeigt haben; Signifikanzniveau zwischen $* \mathrm{p}<0,05$ und $* * \mathrm{p}<0,01$.

Neben der Bewertung der Items spielen innerhalb der Analyse auch Zusammenhänge zwischen Ergebnissen und Aspekten von Integration sowie mit sozialstrukturellen Merkmalen und der Zusammensetzung der Trainingsgruppe eine Rolle. Es zeigte sich, dass signifikante Zusammenhänge zwischen Ergebnissen von Integration bestehen sowie zwischen Ergebnissen und Aspekten von Integration. Dabei zeigte sich zum einen, dass signifikante positive Zusammenhänge zwischen der Befürwortung der Konzepte Segregation und Assimilation bestehen, wohingegen diese beiden Konzepte signifikante negative Zusammenhänge mit den Items zu Pluralismus und Mehrfachintegration aufweisen. Zum anderen zeigten sich auch signifikante positive Zusammenhänge zwischen Pluralismus und Mehrfachintegration. Darüber hinaus ließen sich mehrere signifikante Zusammenhänge zwischen Ausgängen und Aspekten von Integration aufweisen. Die Befürwortung der Assimilation sowie der Segregation scheint mit einer Ablehnung der Einbindung von Zugewanderten einherzugehen, während die Befürwortung von Pluralismus und/oder Mehrfachintegration mit einer höheren Bewertung der Items zur Einbindung und Interaktion einhergeht.

In Bezug auf die Zusammenhänge zwischen Ergebnissen von Integration und sozialstrukturellen Merkmalen sowie der Zusammensetzung der Trainingsgruppe ließ sich Hypothese H1, wonach es einen negativen Zusammenhang zwischen Alter und der 
Unterstützung pluralistischer Konzepte gibt, nicht bestätigen. Es zeigte sich aber, dass die Zustimmung zur Mehrfachintegration mit steigendem Alter sinkt, während die Befürwortung der Segregation steigt. Hypothese H2, wonach es einen Zusammenhang zwischen einem vorhandenen Migrationshintergrund und der Unterstützung pluralistischer Konzepte gibt, konnte bestätigt werden. Hier zeigte sich lediglich beim pluralistischen Ausgang ein signifikanter Effekt. In Bezug auf die Zusammensetzung der Trainingsgruppe zeigte sich ebenfalls ein positiver signifikanter Zusammenhang in Bezug auf dieses Item. Auch Hypothese H3, „Umso höher der Anteil von Personen mit Migrationshintergrund in der Trainingsgruppe, desto stärker werden pluralistische Konzepte unterstützt", lässt sich somit bestätigen. Darüber hinaus weist die Zusammensetzung der Trainingsgruppe auch einen signifikanten positiven Zusammenhang mit der Befürwortung der Mehrfachintegration und einen signifikanten negativen Zusammenhang mit der Befürwortung von Assimilation auf. Somit scheint der Zusammensetzung der Trainingsgruppe eine besondere Bedeutung in Bezug auf die Einstellung zu unterschiedlichen Integrationskonzepten zuzukommen. Dies könnte zum einen daran liegen, dass ab einem bestimmten Punkt Personen mit Migrationshintergrund in diesen Sportgruppen überrepräsentiert sind und die Befragungspersonen einen Migrationshintergrund besitzen. Aber auch der Kontakt zwischen Personen mit und ohne Migrationshintergrund könnte mit zunehmendem Anteil von Personen mit Migrationshintergrund in der Sportgruppe steigen und so einen Einfluss auf die Einstellung zu Integrationskonzepten besitzen.

In Anlehnung an Allport (1954) können Vorurteile durch regelmäßigen Kontakt abgebaut werden und gleichzeitig ein besseres Verständnis für den anderen entstehen. Innerhalb dieser Kontakthypothese geht Allport davon aus, dass Vorurteile gegenüber anderen Personen vor allem auf nationale oder ethnische Zugehörigkeiten zurückzuführen sind (Allport 1954: 34). Dies führt zu einer Differenzierung zwischen „Ingroups“ und „Outgroups“ (Allport 1954: 2934). Die Problematik der Abgrenzung zwischen „Ingroups“ und „Outgroups“ sowie die vorhandenen Vorurteile können jedoch überwunden werden, wenn häufiger Kontakt zwischen den Mitgliedern der unterschiedlichen Gruppen besteht (Allport 1954: 263/264). Dieser Abbau von Vorurteilen kommt zustande, weil Vorurteile zunächst aus Unwissenheit über die andere Gruppe entstehen und durch den Kontakt und die Interaktion mit der „Outgroup“ abgebaut werden können (Pettigrew 1986: 173). Der Kontakt geht somit zunächst mit einem Abbau von Unwissenheit über die andere Gruppe einher und könnte so zum einen zu mehr Verständnis für die andere Gruppe führen als auch zu einer pluralistischeren Einstellung in Bezug auf Integration, da durch den Austausch eine Perspektivenübernahme stattfinden könnte. 
Hypothesen H4 lässt sich nicht bestätigen. In Bezug auf den Bildungsstand zeigt sich ein Widerspruch zur Hypothese, da mit dem Item zum pluralistischen Ausgang von Integration unter Beibehaltung der Kulturen ein negativer Zusammenhang besteht. Beim Item zur Segregation zeigt sich ebenfalls ein signifikanter negativer Zusammenhang mit dem Bildungsstand. Somit werden pluralistische Ansichten, die eine vornherein vorhandene Akzeptanz der Beibehaltung der Kulturen umfassen, mit steigendem Bildungsstand abgelehnt. Gleichzeitig sollen Zugewanderte aber nicht unter sich bleiben, wenn diese sich für die Beibehaltung ihrer Kultur entscheiden.

Insgesamt zeigt sich somit, dass sowohl ein vorhandener Migrationshintergrund als auch die Zusammensetzung der Trainingsgruppe einen positiven Zusammenhang mit der Befürwortung von pluralistischen Integrationskonzepten aufweisen. Darüber hinaus weisen auch die Merkmale Alter und Bildungsstand Zusammenhänge mit der Bewertung von Integrationskonzepten auf, die jedoch nicht den aufgeworfenen Hypothesen entsprechen. 
6.2 Explorative Interviews mit Vereinsvorständen

Die Ergebnisse der explorativen Interviews mit Vereinsvorständen werden in zwei Unterkapiteln dargestellt. Das erste Unterkapitel umfasst die Analyse zu Dimensionen und Aspekten von Integration, während das zweite Kapitel Analysen zu Ergebnissen sowie Adressatinnen und Adressaten von Integration umfasst. Den Abschluss dieses Unterkapitels bildet eine Zusammenfassung und Diskussion der Ergebnisse.

\subsubsection{Analyse der Dimensionen von Integration}

Betrachtet man zunächst die Kodierungen, die in Bezug auf Dimensionen von Integration vorgenommen wurden, zeigt sich, dass diese zu einem großen Teil die Dimension der Interaktion fokussieren. Während für die Dimension der Interaktion 34 Kodierungen vorgenommen werden konnten, sind dies bei der Kulturation 13, bei der Platzierung 12 und bei der Identifikation lediglich 4 (vgl. Abbildung 17).

Abbildung 17: Anzahl der Kodierungen in den explorativen Interviews mit Vereinsvorständen nach Dimensionen von Integration

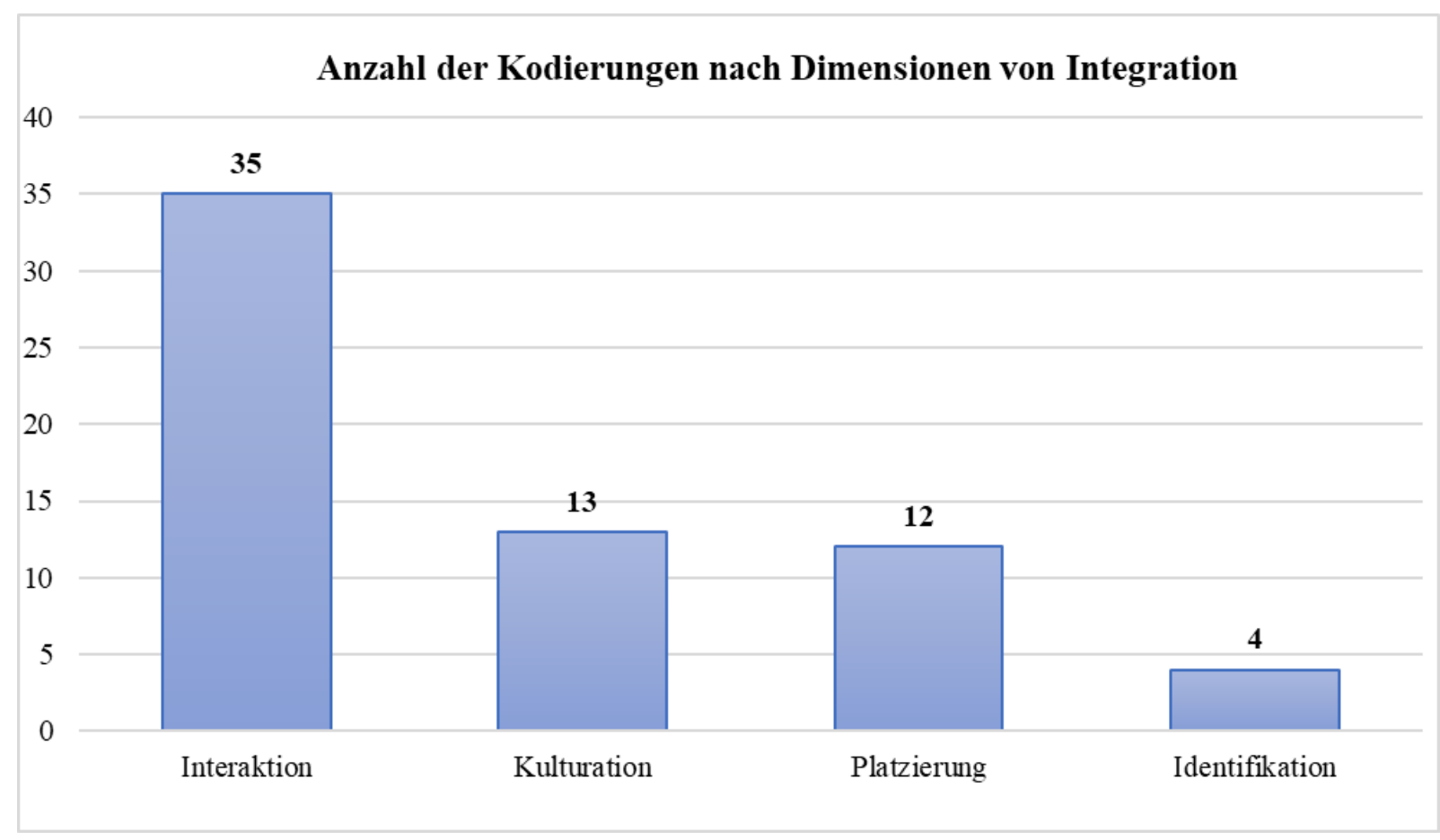

Quelle: Eigene Darstellung.

Dieses Ergebnis zeigt einen Fokus der befragten Vereinsvertreterinnen und -vertreter auf den Bereich der Interaktion.

In Bezug auf die Interaktion beschreiben die Interviewpartnerinnen und -partner Gelegenheiten des Zusammentreffens, den Aufbau interethnischer Kontakte und eine Art 
Anerkennung oder Akzeptanz der „Anderen“, Teilnahmechancen und die Teilnahmebereitschaft als wichtige Aspekte. Anhaltspunkte für die anderen Aspekte, die im Rahmen der Gegenüberstellung der Konzepte im theoretischen Rahmen herausgestellt wurden (Partizipation in Organisationen, politisches Interesse, emotionale und expressive Beziehungen, Sicherung sozio-emotionalen Rückhalts und der Ausgleich konfligierender Interessen), ließen sich in den explorativen Interviews hingegen nicht auffinden.

In Bezug auf die Gelegenheiten des Zusammentreffens betonen die Interviewpartnerinnen und -partner die Bedeutung der Aufhebung von Separierungen. Es sollte somit keine Angebote für bestimmte Personengruppen, wie beispielsweise Geflüchtete, geben, sondern Personen müssen in bestimmten Settings zusammenkommen, die offen für alle gestaltet sind und ihnen die Möglichkeit geben mit anderen in Kontakt zu kommen. Die Aufhebung von Separierungen stellt somit die erste Voraussetzung im Zugang zu Gelegenheiten des Zusammentreffens dar. Hierzu führen zwei der interviewten Personen aus:

- „Also was wir bemerkt haben, wir haben gesagt, aufgrund unserer Tradition, wollen wir keine spezielle Abteilung für Flüchtlinge oder spezielle, was weiß ich, Stunden für Flüchtlinge. Die sollen nicht gesondert sein, sondern die sollen voll integriert sein in allen Abteilungen, wo sie möchten “ (I1: 549-552).

- „,...aber wir sind trotzdem der Meinung, dass wir Integrationsarbeit über den Sport am besten betreiben, indem wir sie zusammenwürfeln und nicht nur für die Flüchtlinge etwas anbieten“(I5: 294-296).

Diese Ausführungen gehen stark mit dem Konzept der Integration in Sport einher und beschreiben Integration als eine Art Einbindung bzw. auch in Form seiner weiten Definition als „die Vervollständigung eines Ganzen, die Wiederherstellung einer Gesamtheit durch das Einführen ihrer notwendigen Bestandteile in ein Ganzes“" (Soeffner/Zifonun 2005: 404).

Mit dieser Einbindung in den Verein, mit Hilfe eines Abbaus von Separierungen, gehen Teilnahmechancen einher, die wiederrum nur wahrgenommen werden, wenn auch eine Teilnahmebereitschaft der $\mathrm{zu}$ integrierenden Personen vorliegt. In Bezug auf Teilnahmechancen und Teilnahmebereitschaft wird zum einen das Angebot, einen Platz im Verein zu erhalten sowie im Verein Aufgaben übernehmen zu können, beschrieben. Damit umfassen die Teilnahmechancen nicht nur die Aktivität im Rahmen des Sportreibens im Verein, sondern auch aktive Möglichkeiten der Partizipation in Form der Besetzung von beispielsweise Ehrenämtern. Zum anderen spielt dabei auch die Initiative der einzelnen Personen, sich aktiv 
einzubringen, eine Rolle. Demnach handelt es sich dabei um einen beidseitigen Prozess, der zum einen Anforderungen an die Vereine als auch an die zu integrierenden Personen stellt:

- „Und Integration heißt für mich, für mich jetzt persönlich, weiß jetzt nicht, wie das jetzt übergeordnet auszusehen hat, dass man einfach reitfremde Menschen und Jugendliche, Kinder zusammenbringt und eben im Verein selbst als sozialer Organisation wirklich jedem quasi eine Aufgabe, einen Platz geben kann, ... “ (I7: 80-84).

- „Also sich einfach auch gut miteinbringen, das ist für mich gelungene Integration, wenn einer von sich aus, auch wenn viel von uns kommt, aber auch selbst bereit ist, von sich aus anzupacken und zu steuern... "(I4: 440-443).

In weiteren Ausführungen wird ein differenzierteres Bild der Einbindung in den Verein deutlich und entstehende Gelegenheiten des Zusammentreffens werden als ein Teil des Prozesses der Integration beschrieben. Somit sind die einzelnen beschriebenen Aspekte nicht losgelöst voneinander zu sehen, sondern in einen Prozess eingebettet, in dem durch die Erfüllung der Aspekte bestimmte Wirkweisen erwartet werden bzw. in der Vergangenheit beobachtet wurden und nun durch die Befragten berichtet werden. So werden in einem Interview Gelegenheiten des Zusammentreffens als Setting, in welchem Integration erst stattfinden kann beschrieben, und ein weiterer Vereinsvorstand beschreibt den Verlauf vom gemeinsamen Training als Gelegenheit des Zusammentreffens hin zum Aufbau von Freundschaften. Demnach stellen Gelegenheiten des Zusammentreffens eine Anforderung in Form eines vorliegenden Settings für Integration dar, das durch den Abbau von Separierungen geschaffen werden kann und zum Aufbau von Freundschaften im Rahmen von interethnischen Kontakten führen kann.

- „Weil nur dann kann man die Integration ja auch machen, weil man sie ja nur machen kann, wenn sie irgendwo sind, wo andere sind, die integ-. Also wo man überhaupt in einen Raum kommt, wo so ein Setting von Integration irgendwie, was auch immer das dann sein mag, stattfindet" (I8: 555-559)

- „Und noch einmal, es gibt nichts Schöneres als wenn Sie bei uns, bei der D-Jugend, CJugend, B-Jugend, sehen und dann sehen Sie Kinder unterschiedlichster Hautfarben, unterschiedlichster Glaubensrichtungen, unterschiedlichster Herkünfte, die zusammen Sport unterhalten, die sich freuen, die sich aufwachsen, die sich dann auch privat treffen und die Freundschaften für das Leben bilden ... “ (I5: 229-234).

Die Bedeutung interethnischer Kontakte für Integration wird in mehreren Interviews betont: „...das ist für mich gelungene Integration, wenn einer von sich aus, auch wenn viel von uns kommt, aber auch selbst bereit ist, von sich aus anzupacken und zu steuern und schnell auch 
Freundschaften, auch außerhalb seines afghanischen Kleinkreises, findet. Dass finde ich Integration, ja.... Und nicht nur Freundschaft unter Deutschen, sondern wirklich mit allen möglichen Nationen “ (I4: 440-446). Kontakte sollten somit kulturelle Grenzen überschreiten und nicht nur zwischen Personen des gleichen Kulturkreises bestehen. Diese Kontakte stellen im Zitat gelungene Integration dar. Im Vergleich zu den Gelegenheiten des Zusammentreffens sind die tatsächlichen interethnischen Kontakte somit auf einer späteren Ebene des Prozesses anzusiedeln. Gleichzeit spricht die interviewte Person hier auch die Initiative der hinzukommenden Person an, sodass diese interethnischen Kontakte ebenfalls als beidseitige Anforderung, an die zu integrierenden Personen und die anderen Personen innerhalb der Gruppe, zu verstehen sind.

Der Aufbau dieser interethnischen Kontakte wird jedoch, auch wenn Gelegenheiten des Zusammentreffens bestehen, nicht als selbstverständlich beschrieben: „Dann ist ja immer nochmal der Punkt, die Leute kommen zu einem Fest, aber wie kriegen wir das hin, dass die Leute auch miteinander kommunizieren und nicht untereinander sitzen. Das ist auch immer nochmal echt ein Riesenproblem. Also wie kriegen wir auch unsere deutschen Mitglieder dazu, dass die auch nicht gemeinsam am Tisch sitzen, sondern auch mal mit einem Geflüchteten sich unterhalten, ne. Also das ist kein, alles kein Selbstläufer" (I6: 245-250). Für interethnische Kontakte scheinen somit, hier in Bezug zum Setting von gemeinsamen Festen, spezielle Aktivierungen auf Seiten der Vereine von Nöten zu sein, die den tatsächlichen Kontakten vorgelagert sind.

Darüber hinaus beschreiben die Personen auch vermutete Wirkungen von interethnischen Kontakten. Hierbei spielt vor allem der Abbau von Vorurteilen durch das gemeinsame Interagieren eine Rolle: „Also man denkt natürlich daran, man hat schon auch seine Vorbehalte, dann tritt man in den Verein an, man trainiert zusammen und man wird eigentlich so, es löst sich eigentlich auf, die Vorurteile lösen sich eigentlich auf und das merkt man auch “ (I8: 122-125).

Ein weiterer Aspekt, der im Rahmen der Interaktion angesprochen wird, ist der der Anerkennung oder Akzeptanz der Anderen: ,Und dann müssen auch alle miteinander zurechtkommen, egal, ob ich ein Kopftuch oder ob ich kein Kopftuch habe, jeder muss den anderen mit Respekt begegnen, sage ich jetzt mal" (I8: 103-106). Im Setting der Interaktion wird erwartet, dass sich die interagierenden Personen und generell die Personen im Verein mit Respekt begegnen. Die Dimension der Interaktion bezieht sich dadurch nicht nur auf die Leistung der zu integrierenden Personen mit einer Gruppe in Kontakt zu kommen sowie auf die 
Leistung des Vereins in Form von Schaffung von Gelegenheiten des Zusammentreffens, sondern auch auf die vorhandene Anerkennung und Akzeptanz durch andere Personen. Dazu ist in manchen Fällen zunächst der Abbau von Vorurteilen und Berührungsängsten durch das Schaffen von Gelegenheiten des Zusammentreffens nötig.

Insgesamt zeigt sich durch den Fokus der Vorstände auf Interaktion ein sehr differenziertes Bild dieser Dimension, welches gleichzeitig einen prozesshaften Charakter aufweist, der sich in Anforderungen und vermutete Wirkungen gliedert. Dieser Prozess wird in der nachfolgenden Abbildung 18 dargestellt. Während die Anforderungen, bis auf den Aufbau interethnischer Kontakte, eher auf Settings in den Vereinen bezogen sind, zielen die Interaktion und auch die vermuteten Wirkungen auf beidseitige Prozesse, also auf die vorhandene Gruppe und die zu integrierenden Personen, ab.

Abbildung 18: Modell der Dimension Interaktion aus Sicht der Vereinsvorstände

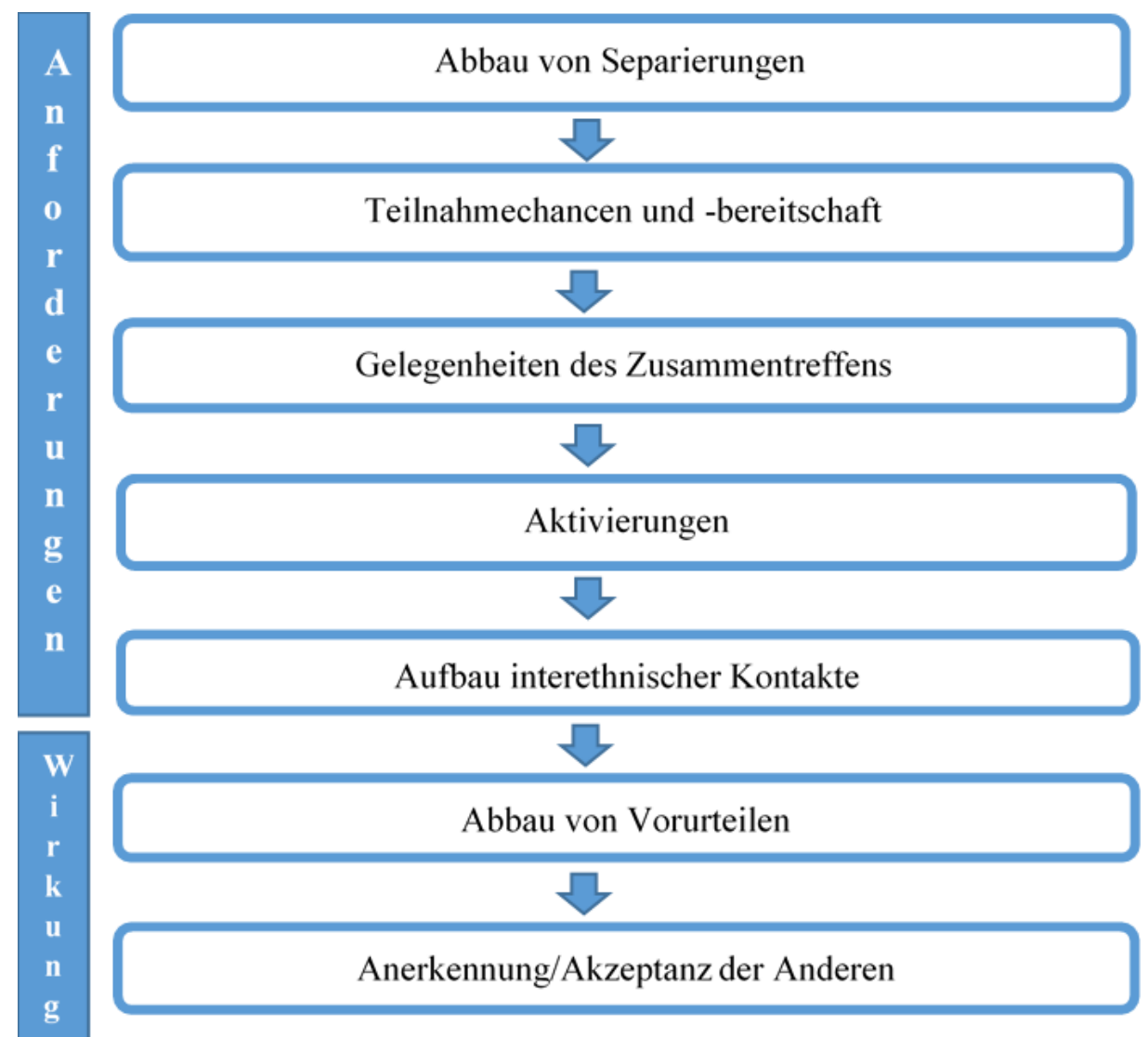

Quelle: Eigene Darstellung.

Bezüge zur Dimension der Kulturation lassen sich vor allem durch die häufigere Ansprache des Themas „sprachliche Fähigkeiten“ herstellen. Neben den sprachlichen Fähigkeiten finden sich auch Passagen, in denen es um weiteres Wissen oder um Kompetenzen für die Bewältigung 
typischer Situationen und die Anerkennung/Internalisierung von Grundnormen geht. Fehlende Aspekte, die im Rahmen der Gegenüberstellung der theoretischen Konzepte auftauchen, sind hier die stark detaillierten Aspekte von Heckmann et al. (2000) (Mediennutzung, Interesse an Politik, Ess- und Trinkgewohnheiten, Einstellung zur Familie und Partnerschaft sowie Bedeutung von Religion).

Im Rahmen des Wissens bzw. Kompetenzen für die Bewältigung typischer Situationen spielen in den Interviews sprachliche Fähigkeiten und das Verständnis für das Vereinswesen und das deutsche System eine Rolle.

In Bezug auf sprachliche Fähigkeiten wird in einem der Interviews die Regelung beschrieben, dass bei Vereinsaktivitäten nur deutsch gesprochen werden darf: „Wir hatten Kleingruppierungen in der Mannschaft, die Afghanen unter sich, die Syrer unter sich und die Ostafrikaner unter sich und dann, jeder hat in seiner Sprache gesprochen und jede Gruppe hat sich von der anderen Gruppe irgendwie beleidigt gefühlt: "Die reden über mich" ja so "Was reden die? Die reden über mich. Die können ja auch nur schlecht über mich reden", weil ja, bestimmt auch ein geringes Selbstwertgefühl und um sowas zu unterbinden, haben wir, der Herr Z. und ich, als wir die Mannschaft übernommen haben, direkt gesagt: "Auf dem Platz, auf dem Sportart 1-Platz darf nur Deutsch gesprochen werden" “ (I4: 122-129).

Diese Regelung soll dazu dienen, die Bildung von ethnischen Gruppen, die eine gemeinsame Sprache sprechen und daraus resultierende Konflikte mit anderen Sprachgruppen zu vermeiden. Darüber hinaus soll eine gemeinsame Sprache dazu führen, dass Barrieren abgebaut und Kontakte zwischen Personen gebildet werden: „Also wir machen das jetzt nur in Ausnahmefällen, dass dann jemand, der die Sprache kennt, versucht ihm zu helfen, wenn es um ganz spezielle Dinge geht oder irgendwie, dass er was braucht für die Behörde oder irgendwas, was ganz schwierig ist auf Deutsch zu erklären, aber ansonsten ist der sicherlich, wie der A2 schon gesagt hat, es ist eine wunderbare Form des Team-Buildings und damit das Team besser zusammenkommt, ja“ (I4: 144-149).

Insgesamt zeigen die Ausführungen zu sprachlichen Fähigkeiten hier, wie bei der Dimension der Interaktion, einen prozesshaften Charakter. Zunächst müssen Regelungen im Verein vorhanden sein und Aktivierungen stattfinden, welche dazu führen, dass eine gemeinsame Sprache gesprochen wird. Die gemeinsame Sprache dient der Vermeidung von Konflikten und führt außerdem zum Abbau von Barrieren, wodurch wiederrum interethnische Kontakte erleichtert werden. 
In einem weiteren Interview werden sprachliche Fähigkeiten außerdem als eine teilweise Aufhebung von Differenz dargestellt: ,,...und man merkt gar nicht mehr, dass er von irgendwo anders her, also er ist, außer dass man es sieht von seiner Hautfarbe ja von seinem Äußeren, hat er aber, er kann perfekt Deutsch er ist schon so weit, dass er so Witze versteht, ja, so doppeldeutige Witze und so weiter" (I4: 431-434).

Weitere angesprochene Aspekte in Bezug auf Wissen oder Kompetenzen für die Bewältigung typischer Situationen, neben der Sprache, ist das Verständnis für das Vereinswesen und das deutsche System. Im Rahmen der Ausführungen zu diesen beiden Elementen werden jedoch lediglich Anforderungen an die $\mathrm{zu}$ integrierenden Personen gestellt und keine vermuteten Wirkungen erläutert:

- „Und aber, wenn man in so einen Verein geht und auf so eine Versammlung geht, dann wird über Dinge geredet, die man auch nicht immer nachvollziehen kann, weil man einfach nicht großgeworden ist in diesem System “ (I6: 410-413).

- „„...die sollen ja auch praktisch lernen, wie das deutsche System funktioniert... “ (I6: 211/212).

Vermutlich lassen sich hier jedoch die gleichen vermuteten Wirkungen wie bei der gemeinsamen Sprache subsummieren. Denn die Kenntnis von Regeln der Gemeinschaft und das Verständnis für ein bestimmtes System können zu einer besseren Nachvollziehbarkeit von Vorgängen führen und so auch zu einem Abbau von Barrieren und der Vermeidung von Konflikten.

Weiter wird auch die Anerkennung/Internalisierung von Grundnormen in einem der Interviews angesprochen. Diese Grundnormen werden in diesem Interview als die „Regeln der Gemeinschaft“ beschrieben: „Und eben auch zu zeigen, wir sind hier 'ne Gemeinschaft, natürlich muss jeder Einzelne die Regeln der Gemeinschaft anerkennen“ (I7: 582-584).

Auch dieser Aspekt wird als Anforderung beschrieben. In einem weiteren Ausschnitt beschreibt eine Person darüber hinaus „Bilder“ aus einem anderen Kulturkreis und führt dazu aus, dass diese Vorstellungen mit Hilfe von Begegnungen abgebaut werden können: „,...das sind wirklich Menschen, die hier hergekommen sind, denen ich gar nichts verübeln kann, weil, ich sag mal, diese totalitäre Regime auf sie eingewirkt haben und irgendwelche Bilder in den Gehirnen verursacht haben über, ich weiß nicht, Frauen, die leicht bekleidet, sind die gleich als Phallusobjekt zu sehen oder dass es nicht akzeptabel ist. Und diese Bilder gilt es mit Hilfe vonvon Treffen mithin- mit Hilfe von Begegnungen abzubauen“" (I5: 361-366). Mit Hilfe von 
Begegnungen kann es somit auch zu einer Anerkennung/Internalisierung der Grundnormen der Aufnahmegesellschaft kommen.

Insgesamt zeigt sich auch bei der Dimension der Kulturation ein prozesshafter Charakter, der sich in Anforderungen und vermutete Wirkungen gliedert. Dabei beziehen sich die Anforderungen zum einen auf Regelungen und Aktivierungen in den Vereinen und zum anderen auf die zu integrierenden Personen, die die gemeinsame Sprache sprechen sowie Regeln kennen und anerkennen sollten. Im Gegensatz dazu zielen die vermuteten Wirkungen, in Form des Abbaus von Barrieren, der Vermeidung von Konflikten sowie das Zustandekommen interethnischer Kontakte, wie bei der Dimension der Interaktion auf beidseitige Prozesse ab. Darüber hinaus zeigt sich im Rahmen der interethnischen Kontakte als vermutete Wirkung auch eine Verflechtung mit der Dimension der Interaktion sowie innerhalb der Kulturation mit den Anforderungen in Form einer gemeinsamen Sprache und der Kenntnis und Anerkennung von Regeln. So kann eine gemeinsame Sprache sowie die Kenntnis und Anerkennung von Regeln zum einen über den Abbau von Barrieren und die Vermeidung von Konflikten zu interethnischen Kontakten führen. Auf der anderen Seite können aber auch interethnische Kontakte zur gemeinsamen Sprache sowie zur Kenntnis und Anerkennung von Regeln führen (vgl. Abbildung 19).

Abbildung 19: Modell der Dimension Kulturation aus Sicht der Vereinsvorstände

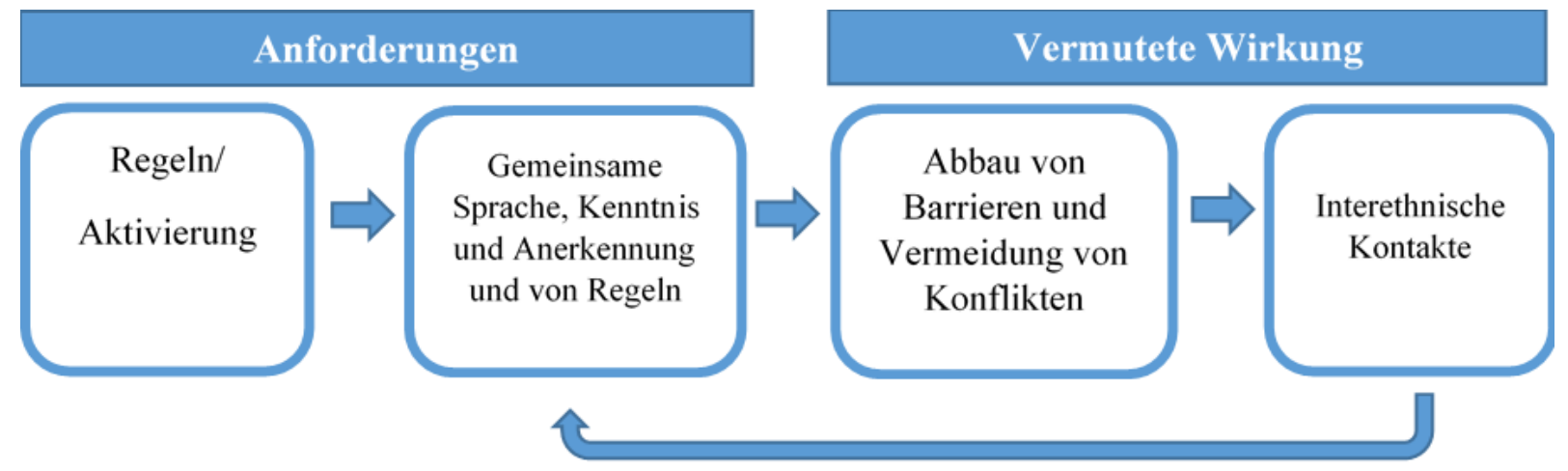

Quelle: Eigene Darstellung.

Aspekte in Bezug auf die Platzierung, die sich in den Interviews finden, sind das Einkommen, die Besetzung bestimmter Positionen und Teilhabe, die rechtliche Gleichstellung, Wohnen und Bildung sowie die berufliche Position. Damit werden die Aspekte Eröffnung sozialer Gelegenheiten, Stellung in der politischen Gemeinschaft, Zugang zum Konsummarkt und Zufriedenheit mit der beruflichen und sozialen Stellung, die in der Gegenüberstellung der 
Konzepte auch dem Bereich der Platzierung zugeordnet wurden, in den analysierten Interviews nicht angesprochen.

Einkommen und Bildung werden im Rahmen der Interviews zum einen als ein Zeichen von Heterogenität zusammen mit anderen Merkmalen (Hautfarbe, Religionszugehörigkeit, Herkunft der Eltern, ...) aufgeführt. Personen soll, laut den Interviewpartnerinnen und partnern, unabhängig von ihrem Einkommen oder ihrer Bildung eine Einbindung ermöglicht werden. Hierzu führt eine Person aus: „Wir haben jetzt aktuell auch wieder, glaube ich, drei Japaner in der Mannschaft: Der eine macht gerade seinen Doktor hier an der Uni, der andere ist glaube ich noch Student. Und die werden dann genauso integriert... Also unabhängig davon, welches Einkommen man hat, welche Hautfarbe, welche Religionszugehörigkeit, aus welchem Land die Eltern kommen, wieviel migrationshistorisch schon dabei ist, ob man frisch angekommen ist oder schon lange hier ist - das hat eigentlich nie eine Rolle gespielt" (I3: 234239).

Zum anderen spielen Aspekte der Platzierung jedoch auch bei der Beschreibung von Personen, die die Vorstände als gelungen integriert ansehen, eine Rolle: „...macht jetzt eine Ausbildung als Bauzeichner, wird wahrscheinlich auch noch studieren ja, lebt mit einer deutschen Freundin zusammen, haben eine eigene Wohnung, ...“ (I4: 413-415). Dies vermittelt den Eindruck, dass Aspekte wie Bildung, Wohnen und die berufliche Situation eher ein Ergebnis von Integration darstellen bzw. dass die Platzierung im Prozess der Integration, aus Sicht der Vereinsvorstände, anderen Dimensionen nachgeordnet ist. Dies wird auch nochmal in der Beschreibung anderer Personen als Beispiel für gelungene Integration deutlich, da dort ein Verweis darauf erfolgt, dass die Personen anfangs wenige Deutschkenntnisse hatten und mittlerweile über Schulabschlüsse verfügen: „, Und die leben jetzt gar nicht mehr in diesen Einrichtungen, sondern haben schon einige so 'ne Art kleine Familien oder WGs mittlerweile. Ja, und die haben auch mit wenigen Deutschkenntnissen bei uns begonnen. Mittlerweile haben sie schon Hauptschulabschluss und der eine sogar schon Realschulabschluss, der wird weiter gehen auf das Abitur zu und sicherlich auch studieren " (I7: 361-366).

Darüber hinaus werden auch die Besetzung bestimmter Positionen und Teilhabe sowie die rechtliche Gleichstellung angesprochen. Diese beziehen sich dabei auf Anforderungen an die Vereine bzw. Aufnahmegesellschaft:

- „...jeder Mensch sollte alle gleichen Rechte und Pflichten haben“ (I4: 676/677).

- „,...und eben im Verein selbst als sozialer Organisation wirklich jedem quasi eine Aufgabe, 
einen Platz geben kann, sodass man sagt, der Verein in sich, mit den Mitgliedern, mit den Aufgaben, die die Mitglieder dann haben, mit den Funktionen, ob's jetzt Jugendteam ist und so weiter, dass die in allen Bereichen gleich teilhaben " (I7: 82-86).

Die letzte Dimension ist die Identifikation. Die wenigen Ausführungen zu dieser Dimension beziehen sich auf die Schaffung von Gemeinschaft und kollektiven Orientierungen sowie auf ein gewisses Zugehörigkeitsgefühl bzw. das Erreichen eines solchen Zugehörigkeitsgefühl mit Hilfe der Stiftung von Identität. Darüber hinaus wird auch das Nachaußentragen einer gewissen Identität angesprochen.

- „Aber wir sind hier, wir sind Verein A, und ich glaube, dass der Verein A dann doch einen guten Teil hat, um hier Identität zu stiften. Dass Menschen ankommen, dass sie sich wohlfühlen und dass sie gemeinsam etwas miteinander machen "(I3: 409-411).

- „Das Schöne ist, dass diese Sportler, die zu uns kommen, sich wirklich wie ein Verein A dann auch definieren und nach außen hin auftreten und das ist genau die Botschaft, die wir möchten, das ist genau das, was wir bei uns ausbilden möchten “(I5: 46-48).

- „Ob es vielleicht ein bisschen altmodisch ist, aber es ist gerade im heutigen Vereinsleben total wichtig, weil da haben wir sehr mit einer verstärkten Individualisierung zu tun und dieses, was ursprünglich traditionell Vereine ausgemacht hat, diese Gemeinschaft, das gibt es ja im Grunde genommen kaum mehr... “(I6: 21-25).

\subsubsection{Analyse von Ergebnissen sowie Adressatinnen und Adressaten von Integration}

Betrachtet man Äußerungen in Bezug auf Ergebnisse von Integration, lassen sich in den explorativen Interviews assimilative sowie pluralistische Ansichten finden. Dabei überwiegen pluralistische Ansichten. In Bezug auf pluralistische Ansichten konnten sechs Textstellen kodiert werden, während assimilative Ansichten in drei Textstellen auftauchen.

Die als pluralistische Ansichten kodierten Segmente beschreiben Formen eines Miteinanders in Vielfalt, Offenheit, Chancengleichheit und die Abschaffung von Unterscheidungen. Im Rahmen der Aussagen zu pluralistischen Ansichten wird deutlich, dass diese zu einem Großteil Leitlinien und Werte der Vereine beschreiben:

- „Also, hier auch Vielfalt ist uns wichtig ja, nicht nur in den Kulturen, sondern auch ja soziale Unterschiede und so weiter, das ist auch wichtig “ (I4: 344/345).

- „Das glücklich zusammen in der Vielfalt auch zu gestalten“ (I7: 500/501). 
Darüber hinaus wird ein Bild von Pluralismus deutlich, das vor allem auf die Ermöglichung des Nebeneinanders ausgerichtet ist. Den Personen sollen Möglichkeiten zur Erreichung ihrer Ziele, faire Chancen und auch Räume zur Entfaltung geboten werden.

- „Wir haben uns dann auch überlegt: Ja, wollen wir denn vorher gucken, wo derjenige wohnt. (---) Nein. Wollen wir gucken, welchen Pass er hat? Nein. Jeder soll eine faire Chance haben" (I3: 352-354).

- „Niemand, es soll eben wirklich so sein, dass alle Frauen und Mädchen, die zu uns kommen, egal eben welche Nationalität, Religion oder Hautfarbe, sollen eben einen Raum haben, um sich mental und psychisch eben entfalten zu können "(I8: 241-243).

- ,Also jeder soll die Möglichkeit haben, seine individuellen Ziele erreichen zu ... “ (I6: 18/19).

Gleichzeitig setzt dieses Miteinander jedoch auch beidseitigen Respekt voraus: „Das Problem auf der Welt ist: Alle werden in denselben Topf geschmissen. Und das ist das Problem. Aber ansonsten ist das bei uns: Wir sollen die respektieren und die sollten auch uns respektieren, ja “ (I3: 846-848). Das Miteinander in Vielfalt wird somit nicht nur durch die Unterstützung bei der Erreichung von Zielen, fairen Chancen und Räumen zur Entfaltung gestaltet, sondern es ist gleichzeitig auf Seiten der Vereine sowie auf Seite der zu integrierenden Personen Respekt füreinander nötig. Dies stellt somit eine Voraussetzung für das Miteinander in Vielfalt dar. Gleichzeitig stellt dieses Miteinander in Vielfalt in den Vereinen vorhandene Leitlinien und Werte dar, wodurch diese Vielfalt als erstrebenswertes bzw. wünschenswertes Ziel der Integration dargestellt wird.

Äußerungen, die als assimilative Ansichten kodiert werden konnten, gehen auf die Dimension der Platzierung und dort auf die Aspekte Bildung und Wohnen ein. Darüber hinaus wird auf die Dimensionen der Kulturation (Sprache) sowie der Interaktion (Beziehungen) eingegangen. In einem Zitat wird Integration hier als das nicht mehr Vorhandensein von Unterschieden beschrieben:

- „Aber zum Beispiel Junge 1, einer unserer größten Beispiele der Integration ja, den wir 2014 abgeholt haben im Flüchtlingsheim und der wirklich klasse, eine klasse Entwicklung auch gemacht hat, der seine Familie, also er kam mit seinen Eltern und vier Geschwistern, vier fünf Geschwistern, ist ja auch egal, kam er her, konnte nicht viel Deutsch, konnte gar kein Deutsch, hat sich aber was das Deutsche angeht, (?Sprachklasse), ist macht jetzt eine Ausbildung als Bauzeichner, wird wahrscheinlich auch noch studieren ja, lebt mit einer deutschen Freundin zusammen, haben eine eigene Wohnung, spielt super Sportart 1... “ 
(I4: 408-415).

- „Ja, einfach, dass es keine und man merkt gar nicht mehr, dass er von irgendwo anders her, also er ist, außer dass man es sieht von seiner Hautfarbe ja von seinem Äußeren, hat er aber, er kann perfekt Deutsch, er ist schon so weit, dass er so Witze versteht ja so doppeldeutige Witze und so weiter" (I4: 431-434).

Im Gegensatz zu den Beschreibungen im Rahmen der pluralistischen Ansichten, die überwiegend Werte im Verein beschreiben sowie die Ermöglichung des Miteinanders, wird in diesen Ausführungen deutlich, dass konkrete Fälle beschrieben werden, die für die Vereinsvorstände Beispiele für gelungene Integration darstellen. Assimilation wird hier nicht als wünschenswerter Ausgang von Integration beschrieben, sondern als ein tatsächlich vorliegendes Ergebnis. Dadurch wird in den Ausführungen der Vereinsvorstände die Diskrepanz zwischen wünschenswerten und empirisch vorliegenden Ausgängen von Integration deutlich, die bereits im Rahmen der Diskussion um Pluralismus und Assimilation ausgeführt wurde.

Neben der Unterscheidung in pluralistische und assimilative Ansichten wird in manchen Interviews auch eine Abgrenzung zu anderen Konzepten des Integrationsdiskurses deutlich. So finden sich Ausführungen, in denen neben der Integration auch das Konzept der Inklusion angesprochen wird, und diese beiden Konzepte für die Vorstände somit getrennte Bereiche darzustellen scheinen.

In Bezug auf die Adressatinnen und Adressaten von Integration kann zwischen Personen, die es zu integrieren gilt, und Personen, die etwas für Integration leisten, unterschieden werden.

Eigenschaften von Personen, die von den Vereinsvorständen mit dem Begriff Integration bzw. mit integrativen Angeboten in den Vereinen in Bezug gesetzt wurden, sind zum einen ein Fluchthintergrund oder ein anderer kultureller Hintergrund:

- „Wir machen auch so Aktionen, um den Flüchtlingen zu helfen, ... “ (I4: 325/326).

- „Und ja, ich denke Integration ist viel mehr. Wir haben mit Menschen aus aller Welt zu tun, wir haben mit Menschen mit Behinderungen zu tun und mit Menschen, die aufgrund ihrer Sexualität vielleicht von der Gesellschaft nicht wahrgenommen werden “ (I4: 475-478).

Darüber hinaus zeigen sich jedoch auch Ausführungen, die ein breiteres Bild der Adressatinnen und Adressaten von Integration vermitteln und auch Eigenschaften wie Alter, Geschlecht, Behinderung und soziale Lage ansprechen:

- „Also wir haben gesagt, dass wir offen sind für alle, unabhängig davon, wie alt sie sind, 
welchen Geschlechts sie sind, welcher Herkunft. Das ist dann das Thema Integration, Behinderung, Fitness, also sowohl für Leute, dass die in der Lage sind, Leistungssport zu treiben, als auch für Leute, die eben nicht so fit sind und einfach Sport treiben wollen, und aber auch ganz stark diesen Inklusionsgedanken vorangeschrieben “(I6: 11-16).

- „Genauso wie wir das haben mit Bildung und Teilhabe. Da gibt es ja auch genügend Jugendliche, die dann eben die Mitgliedsbeiträge nicht zahlen konnten, aber das zahlt die Stadt"(I3: 518-520).

Diese Breite der Differenzkategorien ist ähnlich zum Konzept des Diversitätsmanagements, da in theoretischen Konzepten zu Integration, wie im Verlauf dieser Arbeit bereits dargestellt, in den meisten Fällen der Aspekt des Migrationshintergrundes fokussiert wird.

Somit werden mit dem Begriff der Integration, aus Sicht der Vereinsvorstände, mehrere Gruppen angesprochen und der Begriff wird nicht nur auf Personen mit Migrationshintergrund fixiert. Eine interviewte Person spricht in diesem Zusammenhang übergreifend von Randgruppen: "aber natürlich haben wir das insbesondere auch in Hinblick auf Teilhabe von sogenannten Randgruppen dann gesehen, ne“ (I6: 10/11).

Personen, die in den Interviews als handelnd in Bezug auf Integration dargestellt werden, sind die zu integrierenden Personen, „Einheimische“ sowie Zuständige bzw. Ansprechpartnerinnen und -partner im Verein. In Bezug auf die zu integrierenden Personen selbst, beschreiben die Vorstände das Vorhandensein einer gewissen Offenheit als Anforderung für eine gelingende Integration. Darüber hinaus wird auch eine Wertschätzung der Vorteile in der Aufnahmegesellschaft angesprochen: „Er ist, wie soll ich es erklären, er hat eine deutsche Freundin ja, er hat, ist natürlich ein Glücksfall, also auch, dass er in allen Bereichen halt sehr offen ist. Er ist ein sehr offener Mensch, der alles aufgenommen und weiß auch zu schätzen die Vorteile, die es hier gibt und igelt sich nicht ein, sondern öffnet sich selbst den Menschen gegenüber und will teilhaben" (I4: 436-440).

Auf Seiten der „Einheimischen“ sollte ebenfalls eine gewisse Offenheit vorhanden sein. Anders als bei den zu integrierenden Personen wird hier außerdem eine aktive Komponente in Form des Zugehens auf Geflüchtete angesprochen: „Also wie kriegen wir auch unsere deutschen Mitglieder dazu, dass die auch nicht gemeinsam am Tisch sitzen, sondern auch mal mit einem Geflüchteten sich unterhalten, ne. Also das ist kein, alles kein Selbstläufer" (I6: 248-250). Darüber hinaus spielt auch das Verständnis der anderen Kultur und kultursensitives Wissen eine Rolle. Im Rahmen dieser beiden Voraussetzungen beschreibt eine Person jedoch das Fehlen vieler Grundbegriffe: „Ja, das Verständnis, sagen wir mal, auch von Einheimischen, 
gegenüber auch Flüchtlingen, erfordert mehr Maßnahmen, auch um zu verstehen, was den Kulturkreis anbetrifft, ja. Wenn wir hier jetzt zusammenkommen mit Kindern, Jugendlichen aus Afghanistan, aus Syrien, dann fehlt, fehlen uns sehr, sehr viele Grundbegriffe und ein Verständnis der anderen Kultur" (I2: 197-201).

Die Bedeutung des kultursensitiven Wissens wird auch bei zuständigen Personen im Verein angesprochen: „, Und es ist natürlich so, dass, sag ich mal, es muss im Verein schon auch Ansprechpartner geben, die es gewohnt sind, auch mit Menschen unterschiedlicher Kulturen, umzugehen, die sich einfach auch optisch unterscheiden. Es ist ja nicht nur das Kopftuch, sondern eben auch in dem Gesamten “ (I7: 540-544). Darüber hinaus liegt die Aufgabe dieser Personen im Verein in der Begleitung von Situationen und auch darin, Irritationen zu kommunizieren und moderieren:

- „Man kann dann - und ich glaube, das ist ganz wichtig, auch die Frage, wie begleitet man das - man muss auch schon physisch auch dabei sein, ja “ (I7: 515-517).

- „Also man muss schon sagen, deswegen sag ich, ich ganz gut, man ist anwesend, dass man so Irritationen, die gerad sofort auftauchen, dass man die schon kommuniziert, ja und auch moderiert" (I7: 613-615).

Somit zeigt sich für die zuständigen Personen im Verein eine steuernde Funktion im Integrationsprozess, in der vor allem die Vermittlung zwischen den zu integrierenden Personen und den „Einheimischen“ im Mittelpunkt steht. Integration ist somit, wie in einem der Interviews angesprochen, kein Selbstläufer, sondern bedarf bestimmter Aktivierungen und Maßnahmen sowie einer gewissen Begleitung. 


\subsubsection{Zusammenfassung und Diskussion der Ergebnisse der explorativen}

\section{Interviews mit Vereinsvorständen}

Während im Rahmen der quantitativen Analyse Aspekte und Ausgänge von Integration in ihrer Bedeutsamkeit geordnet werden konnten, kann die qualitative Analyse dazu dienen, angenommene Wirkweisen und Anforderungen in Bezug auf die Aspekte von Integration zu identifizieren. Darüber hinaus können die Beschreibungen zu einzelnen Aspekten, Ausgängen sowie zu Adressatinnen und Adressaten vertiefend herangezogen werden.

Im Rahmen der explorativen Interviews fokussierten die Vereinsvorstände vor allem die Dimension der Interaktion. Dies geht einher mit der zuvor schon beschriebenen Aufteilung in Integration in Sport und Integration durch Sport, bei der gerade der Integration in Sport eine bedeutende Rolle zukommt, während das Konzept der Integration durch Sport eher Kritik erfährt. Die Integration durch Sport würde hier am ehesten der Dimension der Kulturation entsprechen, während die Integration in Sport vor allem im Bereich der Einbindung und Interaktion stattfindet. Des Weiteren ist dieser Fokus auf Interaktion nicht weiter verwunderlich, da in Sportorganisationen Menschen zusammenkommen, um zusammen, oder im Individualsport durch Anleitung, einem Sport nachzugehen. Dieses Ausüben der Sportart in einem Verein geht somit automatisch mit Interaktion einher, welche die Basis des gemeinsamen Sporttreibens bildet. Die Dimension der Interaktion dürfte darüber hinaus auch diejenige sein, die die interviewten Personen am ehesten direkt wahrnehmen können.

Die Darstellungen zu Aspekten und Dimensionen von Integration zeigen, dass im Rahmen der explorativen Interviews eine Vielzahl von Aspekten von Integration angesprochen wird. Darüber hinaus beschreiben die Vereinsvorstände auch vermutete Wirkungen von einzelnen Aspekten, wodurch bei den Dimensionen der Interaktion und Kulturation Modelle der Sichtweisen in Bezug auf Anforderungen und Wirkweisen abgebildet werden konnten. Außerdem wird auch deutlich, dass die Dimensionen der Interaktion und Kulturation in Bezug auf den Aspekt der interethnischen Kontakte Verflechtungen aufweisen. Diese Verflechtungen sowie die Anforderungen und vermutete Wirkungen der Dimensionen der Interaktion und Kulturation sind in der nachfolgenden Abbildung 20 zusammenfassend dargestellt. Dabei werden Anforderungen in blau und vermutete Wirkungen in gelb dargestellt. Der Aufbau interethnischer Kontakte, der im Rahmen beider Dimensionen eine Rolle einnimmt, ist dabei in Bezug auf die Kulturation als vermutete Wirkung zu verstehen und in der Dimension der Interaktion als Anforderung. Innerhalb der Dimension der Kulturation können die 
interethnischen Kontakte wiederum auch auf die Anforderung der gemeinsamen Sprache und Kenntnis sowie Anerkennung von Regeln zurückwirken.

Abbildung 20: Zusammenführung der Ausführungen der Vereinsvorstände zu Dimensionen und Aspekten von Integration

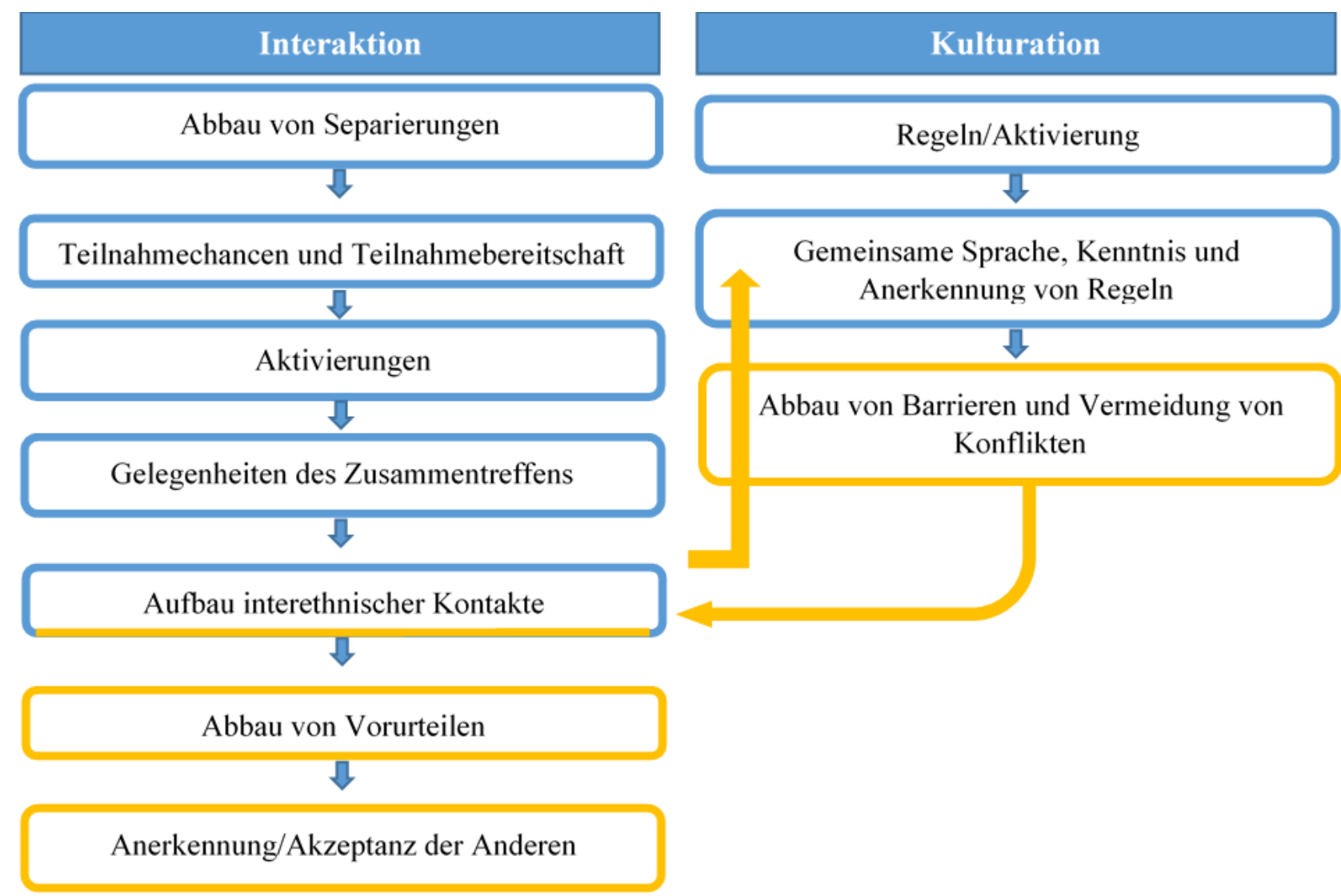

Erläuterung: Blau = beschriebene Anforderungen; gelb = beschriebene Wirkweisen

Quelle: Eigene Darstellung.

Die Dimension der Platzierung scheint eher durch die Darstellung von Teilhabe heterogener Mitglieder in Bezug auf einzelne Platzierungsaspekte (Einkommen, Bildung) sowie durch die Beschreibung von Beispielen gelungener Integration gekennzeichnet zu sein. Dies lässt die Dimension der Platzierung, aus Sicht der Vereinsvorstände, der Interaktion und Kulturation nachgeordnet erscheinen. Der Fokus auf die Dimensionen der Interaktion und Kulturation geht auch mit den Ergebnissen von Lutter (2011) einher. In dieser Studie fokussierten die interviewten Schülerinnen und Schüler ebenfalls eine sozial-kulturelle Perspektive, während strukturelle Aspekte kaum angesprochen wurden. Darüber hinaus finden sich auch in Bezug auf die Identifikation wenig Ausführungen.

Wie in den Untersuchungen von Strahle und Baur (2009) sowie Baur und Mutz (2009), lässt sich im Rahmen der explorativen Interviews, wie auch im quantitativen Teil dieser Arbeit, eine 
Mischung von assimilativen und pluralistischen Tendenzen feststellen. Diese können im Rahmen der Interviewanalyse aber noch weiter beschrieben werden. So umfassen pluralistische Ansichten vor allem eine Ermöglichung des Neben- oder Miteinanders unter der Voraussetzung des gegenseitigen Respekts. Gleichzeitig zeigt sich jedoch auch, dass diese pluralistischen Sichten überwiegend in Leitlinien der Vereine eingebettet sind und so nicht unbedingt aktive Maßnahmen, sondern passive normative Vorstellungen des Miteinanders im Verein umfassen. Im Gegensatz dazu beschreiben assimilative Ansichten Beispiele von ,gelungener“ Integration, wodurch im Rahmen der Interviews die Diskrepanz zwischen wünschenswerten und empirisch vorliegenden Ausgängen von Integration deutlich wird. Dies geht mit den Ausführungen von Hans (2016) einher, die beschreibt, dass im Rahmen von theoretischen Konzepten des Pluralismus oft keine Erklärungsansätze diskutiert werden und unklar bleibt, wie ethnische Pluralität erreicht werden kann. Darüber hinaus wird das empirische Vorkommen der Assimilation in diesen Ansätzen oft ausgeblendet (Hans 2016: 40).

Im Gegensatz $\mathrm{zu}$ den vorgestellten theoretischen Integrationskonzepten sehen die Vereinsvorstände nicht nur Personen mit Migrationshintergrund als Adressatinnen und Adressaten für Integration. Dies könnte darauf zurückzuführen sein, dass die Vereinsvorstände den generellen Einbezug in den Verein als Integration verstehen. Darüber hinaus werden nicht nur die zu Integrierenden, sondern auch Personen, die etwas für Integration leisten müssen, angesprochen. Dies erweitert die Adressatenebene. Diese umfasst somit auch zuständige Personen im Verein, denen eine steuernde Funktion im Integrationsprozess, im Rahmen der Vermittlung zwischen den zu Integrierenden und den „Einheimischen“, zukommt. Und auch „Einheimische“ müssen eine gewisse Offenheit mitbringen, um Integration zu ermöglichen. Diese erweiterte Adressatenebene, die sich nicht nur auf die zu Integrierenden richtet, sondern Integration als gesamtgesellschaftlichen Prozess darstellt, geht mit den vorgestellten Entwicklungen der theoretischen Konzepte einher. Dort zeigte sich vor dem Hintergrund unterschiedlicher gesellschaftlicher und historischer Kontexte und der zunächst starken Dominanz des Assimilationsgedankens im chronologischen Verlauf ein Abkommen von der Idee, Integration wäre nur auf Leistung von Migrantinnen und Migranten begründet und es fanden auch vorhandene Opportunitäten, Leistungen der Gesellschaft sowie emotionale Aspekte Eingang in die theoretischen Konzepte. 
6.3 Vertiefende Leitfaden-Interviews mit Vereinsmitgliedern

Die Ergebnisse der vertiefenden Interviews mit Vereinsmitgliedern werden in vier Unterkapiteln dargestellt. Zunächst folgt ein Kapitel, in dem Unterschiede zu den Vereinsvorständen und zwischen Personen mit und ohne Migrationshintergrund herausgestellt werden. Wie bereits bei den Vereinsvorständen, umfasst das darauffolgende Kapitel die Analyse zu Dimensionen und Aspekten von Integration, während das dritte Kapitel Analysen zu Ergebnissen sowie zu Adressatinnen und Adressaten von Integration umfasst. Abschließend werden die Ergebnisse dieses Unterkapitels zusammengefasst und diskutiert.

\subsubsection{Unterschiede zu Vereinsvorständen und zwischen Personen mit und ohne Migrationshintergrund}

Insgesamt zeigen sich, wie bereits in der quantitativen Analyse, keine deutlichen Unterschiede zwischen Vereinsmitgliedern und Vorständen, weshalb mit Hilfe der vertiefenden Interviews die Beschreibungen der Sichtweisen der Vorstände weiter differenziert werden können, beispielsweise indem im Rahmen der Kulturation anzuerkennende Werte weiter definiert oder die Ausgänge von Integration weiter beschrieben werden. Die überwiegend vorliegenden Ähnlichkeiten in den Beschreibungen sowie aufgefundene Vertiefungen werden in der folgenden Analyse dargestellt.

Weiter zeigen sich im Rahmen der vertiefenden Interviews auch zwischen den interviewten Personen mit und ohne Migrationshintergrund keine deutlichen Unterschiede in Ausführungen $\mathrm{zu}$ Aspekten und Dimensionen sowie Ausgängen von Integration. Der einzige herausstellbare Unterschied besteht darin, dass die beiden Personen mit Migrationshintergrund auch immer wieder Erzählungen ihrer eigenen Erfahrungen mit Integration aufgreifen. Hierzu führt eine Person aus:

- „Aber ich wurde, um das zu sagen, ich wurde sehr gut hier aufgenommen. Mir wurden auch in der Schule hier Deutschkurse angeboten. Also damals gab's halt diese Sprachschulen nicht, ... “(M1: 151-153).

- „Zum Beispiel auch in anderen Fächern, wie also ich wurde schon auch wie so ein Vorbild den anderen Schülern gezeigt, dass ich ohne Deutschkenntnisse zum Beispiel Fächer wie Englisch oder Fächer wie Mathe und so weiter sehr begabt war “ (M1: 165-168).

Bei Personen mit eigener Migrationserfahrung scheint somit das Erleben der eigenen Integration eine bedeutende Rolle für das Sprechen über Integration zu spielen. Dabei stellt die 
Person in diesen Passagen beispielsweise heraus, dass die Integration in die Schule auch ohne Deutschkenntnisse möglich war und er dort positive Erfahrungen gemacht hat.

Im Gegensatz dazu scheint Integration für Personen ohne Migrationshintergrund eher ein abstraktes Konstrukt darzustellen, dass sie, ihrer Einschätzung nach, persönlich nicht betrifft und welches nicht vor dem Hintergrund einer eigenen Erfahrung reflektiert werden kann.

- „Also ich würd' sagen, dass es wichtig ist und dass da auf jeden Fall auch noch mehr gemacht werden kann. Aber so persönlich kann ich dazu jetzt gar nichts sagen, weil es mich nicht betrifft persönlich “ (M4: 82-84).

\subsubsection{Analyse der Dimensionen von Integration}

Auch bei den vertiefenden Interviews mit Vereinsmitgliedern zeigt sich ein Fokus der Beschreibungen auf der Dimension der Interaktion. In Bezug auf die Dimension der Interaktion konnten 27 Kodierungen vorgenommen werden. Darauf folgt die Kulturation mit 24 Kodierungen, die Platzierung mit 11 Kodierungen und die Identifikation mit 3 Kodierungen (vgl. Abbildung 21). Zu beachten ist hierbei jedoch, dass einzelne Fragen im Leitfaden explizit die Dimensionen der Kulturation, Platzierung und Identifikation adressierten, um diese Dimensionen weiter ausführen zu können, da im Rahmen der Vorstandsinterviews vor allem die Dimension der Interaktion umfassend beschrieben wurde. Dennoch wurde in den Beschreibungen innerhalb der vertiefenden Interviews mit Vereinsmitgliedern auch immer wieder die Dimension der Interaktion angesprochen, auf die im Leitfaden nicht explizit Bezug genommen wurde. Demnach wird erneut deutlich, dass vor allem die Dimension der Interaktion einen wichtigen Stellenwert innerhalb der subjektiven Sichtweisen auf Integration einnimmt. 
Abbildung 21: Anzahl der Kodierungen in den Leitfaden-Interviews mit Vereinsmitgliedern nach Dimensionen von Integration

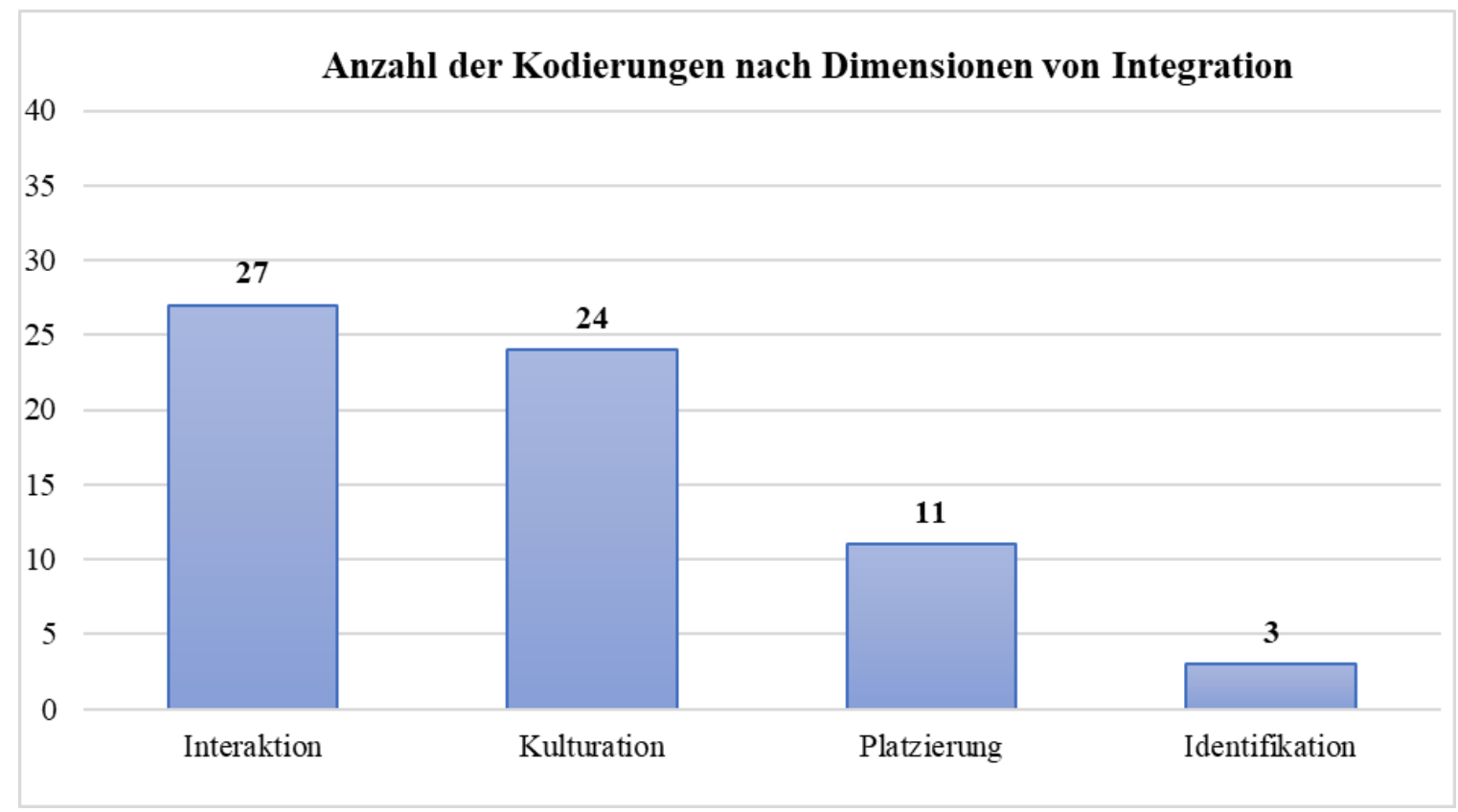

Quelle: Eigene Darstellung.

Bezüglich der Dimension der Interaktion beschreiben die Interviewpartnerinnen und -partner eine Art Anerkennung oder Akzeptanz der „Anderen“, Teilnahmechancen und die Teilnahmebereitschaft als Aspekte von Integration. Die Aspekte Gelegenheiten des Zusammentreffens und Aufbau interethnischer Kontakte, die in den Vorstandsinterviews angesprochen wurden, kommen innerhalb dieser Interviews jedoch nicht zur Sprache. Dies könnte am deutlicheren Bezug der Vorstandsinterviews zu Integration in den Vereinen liegen, wodurch konkrete Abläufe im Verein erläutert wurden. Die vertiefenden Interviews bewegten sich im Gegensatz dazu auf einer allgemeineren Ebene ohne direkten Bezug zum Sport.

Im Rahmen der Anerkennung oder Akzeptanz führen die Vereinsmitglieder aus, dass niemand anders behandelt werden sollte als andere Personen in der Gruppe: „Also, dass man von der Gruppe oder der Gesellschaft aufgenommen wird, nicht anders behandelt wird als die Mitglieder, die da schon sind" (M4: 69/70). Dies ist jedoch nicht als einseitiger Prozess zu sehen, sondern Bedarf einer gewissen Offenheit auf beiden Seiten und somit auf Seiten der Personen, die in die Gruppe einbezogen werden sollen, sowie auf Seiten der bereits vorhandenen Mitglieder der Gruppe. Damit geht auf beiden Seiten auch eine Akzeptanz des Andersseins einher: „....und ja, von beiden Seiten eine Offenheit herrscht und 'ne gewisse Akzeptanz dem Anderssein gegenüber" (M3: 107-109). Diese Ausführungen decken sich mit den Aussagen innerhalb der Interviews mit Vereinsvorständen, in denen im Rahmen der 
Anerkennung und Akzeptanz ebenfalls beidseitige Prozesse, wie beispielweise die gegenseitige Begegnung mit Respekt, beschrieben wurde.

Darüber hinaus beschreiben die Vereinsmitglieder auch die Aspekte der Teilnahmechancen und Teilnahmebereitschaft, welche erneut auf einen beidseitigen Prozess der Integration hindeuten. Zum einen sind im Rahmen der Teilnahmechancen Angebote nötig und darüber hinaus sollte niemand von vorhandenen Möglichkeiten ausgeschlossen werden: „Also, dass man nicht ausgeschlossen wird von den Möglichkeiten " (M4: 48/49). Eine Person beschreibt dabei auch eine Art präventiven Charakter von Teilnahmechancen im Rahmen eines Sportangebotes: „Weil das Problem ist, wenn die viel draußen hängen, dann kommen die auf schlechte Gedanken: Kriminell zu werden oder zuhause einfach unnötig zu trinken und so weiter. Und das wollen wir vermeiden “ (M1: 96-98).

Zum anderen muss für die Wahrnehmung dieser Teilnahmechancen auch eine gewisse Teilnahmebereitschaft vorhanden sein, die teilweise auch als ein gewisses Maß an Motivation dargestellt wird. Diese wird in den geführten Interviews mehrfach betont:

- „Aber einfach eine Bereitschaft zeigen, dass man gerne am Leben teilhaben möchte “(M5: 20/21).

- „Also von der Person aus auf jeden Fall Angebote, die eventuell zur Integration bestehen, anzunehmen. Also beispielsweise, wenn Personen aus dem Ausland kommen, Sprachkurse annehmen oder auch irgendwelche Sportkurse. Genau, da halt 'ne Motivation zeigen, sich auch integrieren zu wollen " (M4: 14-17).

Auch diese Ausführungen decken sich mit den Aussagen innerhalb der Interviews mit Vereinsvorständen. Eine interessante Ergänzung ist hierbei jedoch, dass in den Ausführungen der Vereinsmitglieder auch angesprochen wird, dass Vorurteile oder Stereotype dazu führen können, dass trotz Teilnahmebereitschaft die tatsächliche Teilnahme und damit auch die Integration erschwert wird. Dazu führt eine Person aus, dass selbst wenn man jegliche Bereitschaft und Integrationswillen zeigt, die Gesellschaft dieser entgegenstehen kann. Demnach ist auch die Teilnahmebereitschaft wiederrum davon abhängig, auf eine gewisse Offenheit in der Gesellschaft zu treffen.

- „Dass das aber mit gewissen Hindernissen beziehungsweise Schwierigkeiten verbunden ist/ sein könnte, aufgrund von unterschiedlichen Vorannahmen bezüglich auch Vorurteilen, Stereotypen, genau“ (M5: 4-7).

- „,... weil man kann ja noch so sehr versuchen sich zu integrieren. Wenn auf der anderen 
Seite nur 'ne Mauer steht, die du nicht durchbrechen kannst, dann kann auch jede noch so gut gemeinte Integration nicht gelingen... “(M5: 28-30).

Auch im Rahmen dieser Interviews lassen sich Bezüge zur Dimension der Kulturation herstellen. Im Rahmen des Wissens bzw. Kompetenzen für die Bewältigung typischer Situationen spielen, wie schon bei den Vereinsvorständen, vor allem sprachliche Fähigkeiten eine Rolle. Diese sollten nach Ansicht der Interviewpartnerinnen und -partner vorhanden sein bzw. zumindest ein Interesse daran, diese zu erlernen. Auf der anderen Seite müssen aber auch entsprechende Angebote vorhanden sein, die das Erlernen der Sprache ermöglichen.

- „Also wenn es jemandem leichtfällt, auf Menschen zuzugehen beziehungsweise, dass Sprache auch ein ganz wichtiger Teil davon ist und dass eventuell man zunächst die Sprache versucht zu lernen ... “(M5: 59-62).

- „Meiner Meinung nach sollten sie natürlich eine gewisse Offenheit mitbringen, dass sie sich überhaupt für die, für das Land und die Kultur, in die sie hineinziehen, interessieren und auch bereit sind, die Sprache zu lernen ... “ (M2: 10-12).

- „Zum Beispiel, dass es Zugang zu Sprachkursen gibt oder 'n leichten Zugang zu Sprachkursen, ... “(M3:23/24).

Weiter ist im Rahmen der Kulturation auch die Anerkennung/Internalisierung von Grundnormen in den Interviews von Bedeutung. Hier werden überwiegend Anforderungen an die Personen formuliert, die es zu integrieren gilt. Diese Anforderungen umfassen zum einen das Interesse an bzw. die Annahme von Gepflogenheiten, Werten und Regeln des Landes. Dies bedeutet jedoch nicht, dass die eigene Meinung oder Einstellung komplett aufgegeben werden sollte. Auf der anderen Seite beschreibt eine Person auch, dass Personen aus anderen Ländern offen mit ihrem kulturellen Hintergrund umgehen und Elemente davon präsentieren sollten. Somit zeigt sich hier, ergänzend zu den Interviews mit Vereinsvorständen, dass die Anerkennung/Internalisierung von Grundnormen nicht nur auf die Normen im Aufnahmeland bezogen sein muss, sondern dass auch die Anerkennung von anderen Kulturen bzw. ein gewisses Interesse dafür in der aufnehmenden Gesellschaft sowie ein gemeinsamer Austausch darüber eine Rolle spielen kann, sofern diese kulturellen Elemente von den Personen aus anderen Kulturkreisen auch nach außen getragen werden.

- „Also wieder zu dem Beispiel, wenn man aus dem Ausland kommt, würd' ich sagen, dass vor allem Werte von - die Werte, die in dem Staat gelten, anerkannt werden sollten " (M4: $52 / 53)$.

- „ „...man sollte bereit sein zum Beispiel auch Regeln und kultureigene, was man zeigen 
möchte, auch den anderen zu präsentieren " (M1: 65/66).

- „,...wenn man zum Beispiel, wir sind in einer Gruppe und wir sagen, sagen wir mal wir haben Weihnachtsfeier, und dann zum Beispiel auch ein Flüchtling dabei und dann sagen wir: ,Bei uns ist es hier Weihnachten, was ist bei euch? Welche Feste gibt es bei euch“" (M1: 26-30).

Insgesamt zeigt sich auch, dass keine vollständige Übernahme der Werte eingefordert wird. Die zu integrierenden Personen sollten jedoch versuchen, offen für die Werte des Aufnahmelandes zu sein und auch eine Einstellung zu diesen Werten zu finden. Darüber hinaus sollten zumindest Regelungen, die den gesetzlichen Rahmen betreffen, eingehalten werden.

- „Und ich bin der Meinung, dass man natürlich seine eigene Meinung mitbringen darf, aber auch offen für das sein sollte, was im aufnehmenden Land herrscht. Also wenn hier offen darüber diskutiert werden kann, finde ich, sollte man auch zumindest versuchen offen dafür zu sein und 'ne Einstellung dazu zu finden" (M2: 66-70).

- „Ansonsten sollte jeder eigentlich so freiheitlich leben können, wie er es möchte, solange es wie gesagt in den bestimmten Regeln oder in dem bestimmten Rahmen gesetzlich funktioniert" (M3: 109-111).

Darüber hinaus sollte innerhalb der vertiefenden Interviews herausgestellt werden, was unter Grundnormen oder Werten verstanden wird, um diesen Begriff differenzierter fassen zu können. Es zeigte sich zunächst, dass Werte und Grundnormen als ein kontextabhängiges Konstrukt gesehen werden, das immer in Bezug zum „Aufenthaltsort“ gedacht werden muss. Dabei können sich Werte jedoch auch innerhalb einer Gesellschaft unterscheiden.

- „Also ich glaube, dass das immer sehr stark davon abhängt, von welchem Land man spricht. Oder von welcher Weltbevölkerung“ (M5: 66/67).

- „Das ist natürlich ganz schwierig, weil auch innerhalb der Gesellschaft verschiedene Werte herrschen, ... “(M2: 61/62).

Als wichtige Werte nennen die Interviewpartnerinnen und -partner Toleranz, Weltoffenheit, die Gleichberechtigung von Mann und Frau, Nächstenliebe, Akzeptanz von Homosexualität und weiteren in anderen Regionen tabuisierten Themen, Gleichheit, freiheitliches Leben und auch das Grundgesetz mit den darin befindlichen Werten.

- „Also wenn wir uns jetzt mal auf den Westen beziehen, wo ja auch Deutschland dazugehört, dann würde ich sagen, dass die Werte mitunter sind: Toleranz, also sowas wie Weltoffenheit, und dass es keine Diskriminierung gibt. Und auch Geschlechtergerechtigkeit 
spielt da ja häufig mit rein, weil das ja auch gegen diese Menschen verwendet wird. Ja, also sowas wie Nächstenliebe - ich weiß nicht, ob das ein Wert ist, aber das würde ich da mit reinziehen. Und einfach Gleichheit aller Menschen, das ist, glaube ich, wichtig“ (M5: 6773).

- ,es geht in erster Linie darum, Mitmenschen zu akzeptieren und zu respektieren und wenn man jetzt mal von Deutschland spricht, gibt's hier irgendwie die Gleichberechtigung der Frau und es gibt 'ne offene Auslegung von Homosexualität und gewissen Themen, die in anderen Regionen der Welt einfach noch stark tabuisiert sind “ (M2: 62-66)

- „Also das Wichtigste ist auf jeden Fall das Grundgesetz. Das ist das, worauf sich alles berufen sollte. Und dann gibt's halt noch, würde ich sagen so Gepflogenheiten und so. Aber das ist eben auch zum Beispiel von Bundesland zu Bundesland unterschiedlich. Dann halt einfach so allgemeine Sachen: Dass man keinem anderen Schmerzen zufügen sollte, dass jeder freiheitlich leben sollte und freiheitlich denken sollte und so. Ja, das ist halt einfach auch sehr westlich" (M3: 63-68)

Interessant ist dabei, dass die meisten Interviewpartnerinnen und -partner die Frage nach konkreten Werten zunächst als schwierig zu beantworten einschätzen. Bei den Ausführungen finden sich dann jedoch einige Übereinstimmungen, sodass trotzdem ein gewisser Konsens über die wichtigsten Werte vorhanden zu sein scheint.

In den eben aufgeführten Zitaten wird auch deutlich, dass diese Werte als westlich angenommen werden.

- „Also wenn wir uns jetzt mal auf den Westen beziehen, ... “ (M5: 67).

- „Ja, das ist halt einfach auch sehr westlich“ (M3: 68).

Diese Abgrenzung wird auch in einem weiteren Zitat deutlich, in welchem Bezug auf christliche Werte genommen wird: „Also das ist jetzt hier in Deutschland beispielsweise christliche Werte. Nicht, weil man sagt, dass das von der Religion kommt, aber dass man halt keine Straftaten begeht und nett ist und genau" (M4: 54-56).

Es zeigt sich somit, dass diese Werte, in Differenz $\mathrm{zu}$ anderen, als nicht-westlich wahrgenommene Werte gedacht werden. Dadurch ergibt sich, dass implizit nur Personen aus nicht-westlichen Regionen als Adressatinnen und Adressaten dieser Ebene der Integration angenommen werden. Die Anerkennung/Internalisierung von Grundnormen bezieht sich damit hauptsächlich auf Personen aus anderen Kulturkreisen und nicht beispielsweise auf Personen aus Europa. 
In Bezug auf die Platzierung sollte im Rahmen der vertiefenden Interviews geklärt werden, auf welche Personen sich diese Platzierungsaspekte beziehen und auch, welche Wirkweisen im Rahmen dieser Dimension angenommen werden. Es zeigt sich, dass Aspekte der Platzierung, wie der Bildungstand, die berufliche Stellung und der finanzielle Hintergrund, vor allem auf Seiten der zu Integrierenden eine Rolle spielen. Dabei werden diese Aspekte jedoch nicht als Voraussetzung gesehen. Vielmehr nehmen die Interviewpartnerinnen und -partner an, dass ein höherer Bildungsstand oder eine gute berufliche Stellung die Integration in die Gesellschaft erleichtern können.

- „Und von daher denke ich schon, dass gute Bildung und guter finanzieller Background dazu beitragen können, dass die Leute einfach sich besser und schneller integrieren - dass sie sich schneller wohlfühlen. Und auch von der Gesellschaft - also dort, wo sie integriert werden sollen - auch besser angenommen werden “ (M3: 83-87).

- „Ich glaube, je höher der Bildungsstand ist, desto einfacher ist es wahrscheinlich, sich zu integrieren. Ich finde aber nicht, dass er ausschlaggebend für eine erfolgreiche Integration ist" (M2: 44-46).

Diese Annahme stützt sich dabei überwiegend darauf, dass Personen mit einem höheren Bildungsstand oder einer besseren beruflichen Stellung besser in der Gesellschaft angenommen werden, da mit diesen Aspekten gewisse Nutzenerwartungen und auch Stereotype verbunden werden. Die Interviewpartnerinnen und -partner nehmen an, dass Personen, die durch ihren Bildungsstand oder Beruf einen Nutzen für die Gesellschaft haben, auch eher angenommen werden. Darüber hinaus beschreibt eine interviewte Person das Beispiel der „Schmarotzer“ als vorhandenen Stereotyp in der Gesellschaft, der mit einem niedrigen Bildungsstand oder einer niedrigen beruflichen Stellung verbunden wird.

- „Aber es ist wahrscheinlich leichter, jemanden mit einer guten beruflichen Qualifikation zu integrieren, weil davon die Gesellschaft in beruflicher Hinsicht ja auch 'n Nutzen trägt" (M4: 63-65).

- „Also ich glaube, dass der Bildungsstand dann, wenn man einen guten hat, die Integration erleichtert [...] Also häufig kommt ja dieses Vorurteil der Schmarotzer/ der Schmarotzerinnen usw. und wenn man halt eine gewisse Bildung mitbringt, dann wird mit Bildung immer ein gewisses Verhalten verbunden. Und dass dadurch Integration halt einfach leichter wird, weil dadurch signalisiert wird „Ok, ich bin ja bereit zu arbeiten/ etwas zu leisten und das würde ich gerne auch den anderen Menschen zur Verfügung stellen “. Also dass es einfach ein besseres Bild ist, wenn man davon ausgeht, dass diese 
Menschen dann auch Geld bringen und nicht nur Geld wollen. Das ist ja häufig dieses Vorurteil. Und genau so ist es ja auch mit der beruflichen Stellung. Also wenn man natürlich einen guten Beruf ausführt, mit 'nem gewissen Ansehen, der mit der Gesellschaft verknüpft ist, dann fällt es natürlich leichter da irgendwie einzusteigen und Anschluss zu finden. Also als wenn man jetzt zum Beispiel einen niederen Beruf ausübt, der ohnehin einen schlechteren Ruf hat, dann ist das natürlich ja, einfach problematischer, da Anschluss zu finden und Akzeptanz" (M5: 75-91).

Somit zeigt sich, dass die Aspekte der Platzierung in erster Linie keine direkten Anforderungen an die zu integrierenden Personen darstellen. Jedoch können diese Aspekte, je nachdem welchen Bildungsstand oder welche berufliche Stellung die jeweilige Person innehat, förderlich oder hinderlich für die Integration in die Gesellschaft sein. Bereits bei den Interviews mit den Vereinsvorständen zeigte sich, dass die Dimension der Platzierung den anderen Dimensionen im Prozess der Integration eher nachgeordnet zu sein scheint. Dort verwiesen die Ausführungen jedoch eher darauf, dass die Platzierung ein Ziel von Integration sein könnte. Jedoch wurden dort auch Beispiele angeführt, in denen Personen mit wenigen Sprachkenntnissen und ohne Schulabschluss im Zeitverlauf Schulabschlüsse erreichten. Somit scheint es für die Auslegung der Platzierung einen Unterschied zu machen, ob man die Wirkung des Bildungsstandes bei Ankunft im Aufnahmeland oder den Bildungsverlauf im Aufnahmeland betrachtet.

Bezüglich der Dimension der Identifikation befanden sich in den Vorstandsinterviews die wenigsten Ausführungen, weshalb auch diese Dimension im Rahmen der vertiefenden Interviews weiter differenziert werden sollte. Die Interviews mit den Vereinsvorständen hatten bereits gezeigt, dass sich die Dimension der Identifikation auf die Schaffung von Gemeinschaft und kollektiven Orientierungen sowie auf ein gewisses Zugehörigkeitsgefühl bzw. das Erreichen eines solchen Zugehörigkeitsgefühl bezieht. Im Rahmen der vertiefenden Interviews sollte nun hinterfragt werden, wie ein solches Zugehörigkeitsgefühl zustande kommen kann.

Die Interviewpartnerinnen und -partner schildern hier eine Verknüpfung mit der Dimension der Interaktion. Im Rahmen des Zugehörigkeitsgefühls wird an mehreren Stellen deutlich, dass dieses überwiegend in Bezug zu anderen Personen gedacht wird. So beschreibt eine Person das Zugehörigkeitsgefühl als ein Gefühl des sich „gut aufgehoben Fühlens“, wenn beispielsweise Freundschaften geknüpft wurden. Darüber hinaus spielt es auch eine Rolle, dass Personen sich akzeptiert und respektiert fühlen. Diese Faktoren werden auch als notwendig dafür dargestellt, dass ein Zugehörigkeitsgefühl überhaupt erst entstehen kann.

- „Also ich glaub' Zugehörigkeitsgefühle erhält man dann, wenn man sich gut behütet und 
aufgehoben fühlt und vielleicht auch, wenn man Freundschaften geknüpft hat, einfach weil man sich gut behandelt fühlt [...] Aber ich glaube vorrangig ist erst mal, dass man sich akzeptiert und respektiert fühlt innerhalb einer Gesellschaft. Und dass das einfach dazu führt, dass ich mich überhaupt zugehörig fühlen kann, weil ich merke, Ok, die Menschen nehmen mich so, wie ich bin und das in Ordnung so “" (M5: 94-102).

- „ „...jetzt beispielsweise - in dem neuen Land wohl fühlen oder in der neuen Gruppe und das Gefühl haben, auch akzeptiert zu werden, ... “ (M4: 40-42).

- „Also dass man von der Gruppe oder der Gesellschaft aufgenommen wird, nicht anders behandelt wird als die Mitglieder, die da schon sind. Ja und halt auch dieselben Möglichkeiten hat und gut behandelt wird einfach und auch selbst von sich sagt, dass man sich da wohlfühlt und Ansprechpartner hat" (M4: 69-72).

Das Zugehörigkeitsgefühl wird darüber hinaus auch als ein individueller Faktor im Rahmen der Integration angesehen, welcher, selbst wenn Personen als integriert gesehen werden, nicht vorhanden sein muss.

- „Ich glaub', das ist 'ne ganz individuelle Sache. Weil man kann ja noch so gut integriert sein und sich trotzdem nicht zugehörig fühlen, weil irgendwie was fehlt oder weil es einfach doch nicht so ist, wie man es davor gewohnt war" (M5: 96-99).

\subsubsection{Analyse von Ergebnissen sowie Adressatinnen und Adressaten von Integration}

Auch in den Interviews mit Vereinsmitgliedern zeigt sich eine Mischung aus assimilativen und pluralistischen Ansichten. Dabei wird die Ausgestaltung dieser Ergebnisse von Integration jedoch weiter differenziert. Während bei den Vereinsvorständen Aussagen zu beiden Konzepten aufzufinden waren, und das auch innerhalb der einzelnen Interviews, zeigen sich hier eher Verknüpfungen zwischen Assimilation und Pluralismus.

Zum einen führen die Interviewpartnerinnen und -partner aus, dass Integration nicht bedeutet, dass Personen ihre Kultur ablegen sollten. Vielmehr sollte es zu einer Anpassung in bestimmten Bereichen kommen, während Differenzen in anderen Bereichen akzeptiert werden. Dieses Zusammenspiel von Anpassung und Akzeptanz soll in einer Art Gleichheit münden, die sich auf einen gemeinsamen Werte- und Gesetzesrahmen sowie auf eine gemeinsame Sprache bezieht. In einem der Interviews wird in Bezug darauf von einem gemeinsamen Nenner gesprochen.

- „Also das soll nicht heißen, dass sie ihre eigene Kultur ablegen sollen, aber sich dem Neuen 
auch offen Gegenüber zeigen sollen und anpassen“"(M2: 14-16).

- „Also ich finde nicht, dass es heißt, dass alle Mitglieder komplett gleich sind, damit die Integration funktioniert hat, aber so, dass die Gesellschaft reibungslos funktioniert. Also dass, um auf die Werte zurückzukommen, dass die angenommen werden und auch eingehalten werden, aber halt auch individuelle Merkmale der Personen trotzdem noch bestehen dürfen “ (M4: 75-79).

- „Was jetzt auch nicht bedeutet, dass man alles akzeptieren muss, aber dass man einfach auch Andersartigkeit und andere Gedanken auch akzeptiert, sofern das natürlich alles mit den wichtigsten Grundpfeilern in diesem Land oder in der Gesellschaft zu vereinbaren ist. Also solange das Grundgesetz eingehalten wird und vielleicht auch bestimmte Gepflogenheiten - das, denke ich, macht irgendwie schon Sinn, dass Leute sich bemühen auf einen Nenner zu kommen, weil das einfach auch Menschen zusammenbringt “ (M3: 9399).

- „Für mich wäre es zumindest schon mal ein erster Schritt, wenn man eine gemeinsame Sprache sprechen würde und sich auf einige grundlegende Werte einigen würde. [...] Andererseits ist es natürlich auch eine Herausforderung, wenn das mit Menschen aus völlig unterschiedlichen Kreisen irgendwie so heruntergebrochen werden muss, dass es auf alle zutrifft" (M2: 76-92).

Die vorangegangenen Beispiele zeigen, dass im Rahmen der Integration somit eine Wechselbeziehung anzunehmen ist, innerhalb derer beide Seiten aufeinander zugehen müssen, um Integration zu ermöglichen. Bezogen auf den gemeinsamen Werterahmen, der als Ziel von Integration dargestellt wird, existiert ein flexibler Prozess der gegenseitigen Anpassung, der über die Zeit zu gesellschaftlichen Veränderungen führen kann, indem beispielsweise Normen und Werte neu ausgelegt werden.

Mit dieser Verknüpfung von Assimilation und Pluralismus geht auch einher, dass die Gesellschaft liberal sowie sozial ausgestaltet ist und die Personen innerhalb der Gesellschaft einen Umgang miteinander gefunden haben, der Differenzen akzeptiert und auflöst.

- „Also gelungene Integration sagt für mich aus, dass die Leute verstanden haben, miteinander umzugehen und dass es da keine Unterschiede gibt. Eine Gesellschaft, in der alle integriert sind, die sollte auf jeden Fall sehr liberal sein, würde ich sagen. [...] Weil ansonsten funktioniert das nicht. Sozial ausgerichtet, ja, einfach ein Miteinander pflegen und auch einsehen, dass die eine Gruppe vielleicht mehr für die andere Gruppe sorgen muss beziehungsweise dafür aufkommen muss, weil es manchen Menschen nicht möglich ist “ 
(M5: 107-113).

In Bezug auf die Adressatinnen und Adressaten von Integration kann auch hier zwischen Personen, die es $\mathrm{zu}$ integrieren gilt, und Personen, die etwas für Integration leisten, unterschieden werden.

Eigenschaften von Personen, die mit dem Begriff der Integration von den Vereinsmitgliedern in Bezug gesetzt werden, beziehen sich, wie schon bei den Vereinsvorständen, vor allem auf einen anderen kulturellen oder Fluchthintergrund. Dabei wurde in den vorherigen Ausführungen bereits deutlich, dass sich dieser kulturelle Hintergrund vor allem auf Personen aus nicht westlichen Staaten bezieht, bei denen zudem ein anderer Werterahmen angenommen wird. Dies stellt eine Erweiterung zu den Interviews mit Vereinsvorständen dar.

- „Da denk' ich zuerst daran, dass gewisse Leute neu in ein Land kommen... “ (M5: 2).

- „An Migranten und aus aktuellem Anlass natürlich auch Flüchtlinge“ (M2: 8).

- „Wenn ich ,Integration' höre, denke ich an Menschen, die in andere Länder (korrigiert sich), in fremde Länder oder fremde Kulturen einwandern oder - ja - ein neues Leben beginnen“ (M3: 2-4).

- „Es geht darum, Menschen aus einem anderen Kulturraum zu integrieren“ (M2: 2/3).

Darüber hinaus zeigen sich auch Ausführungen, die ein breiteres Bild der Adressatinnen und Adressaten von Integration vermitteln und sich auf Personen mit Behinderungen oder gesundheitlichen Einschränkungen sowie generell auf Minderheiten beziehen.

- „Ich denk' jetzt konkret direkt an Minderheiten, ... “ (M4: 8).

- „,...wie auch an Personen mit gesundheitlichen Einschränkungen oder körperlichen Behinderungen, weil es ja auch eine gewisse Integrationsarbeit benötigt, um am alltäglichen Leben teilzunehmen, da die Mehrheit einfach gesundheitlich gesund ist" (M5: 13-16).

In einem weiteren Interview wird außerdem allgemein von Personen gesprochen, die in eine Gruppe oder Gesellschaft aufgenommen werden sollen, was einem breiten Bild von Integration ohne definierten Adressatenkreis entspricht.

- „Also einfach auch Anschluss finden in einer Gruppe oder eine Gesellschaft“ (M4: 4/5).

Somit liegt auch bei den Vereinsmitgliedern eine breitere Auslegung des Integrationsbegriffs in Bezug auf seine Adressatinnen und Adressaten vor, der nicht nur auf Personen mit Migrationshintergrund fixiert ist. Dies zeigte sich auch bei den Interviews mit Vereinsvorständen. 
Als handelnde Personen werden von den Vereinsmitgliedern in Bezug auf Integration ebenfalls mehrere Adressatinnen und Adressaten angesprochen, die erneut eine gewisse beidseitige Leistung von den zu Integrierenden und den Personen, die bereits Teil der Gesellschaft sind, für Integration betonen.

- „Also, dass Integration immer von zwei Seiten ausgeht: Sprich von Leuten, die zum Teil bereits in einem Land wohnen oder Leuten, die keine Einschränkungen haben, dass diese auch offen auf die Menschen zugehen und denen dadurch auch Integration erleichtern [...]. Also es ist ein Wechselspiel auf jeden Fall “(M5: 25-34).

- Ich denke, dass alle Menschen dazu beitragen müssen“(M3: 16).

- „Klar, die Zivilgesellschaft ist da auch gefragt und auch der aufnehmende Staat“ (M2: 19).

Darüber hinaus sprechen mehrere Interviewpartnerinnen und -partner auch die Verantwortung der Politik in Bezug auf Integration an. Konkrete staatliche Maßnahmen, die dabei eine Rolle spielen, sind vor allem Integrationsangebote, wie beispielsweise Sprachkurse, aber auch die Bereitstellung von Informationen über entsprechende Angebote und unterstützende Maßnahmen bzw. zuständige Institutionen.

- „Also ich würd' jetzt konkret erst mal sagen, von der Regierung. Also auch aus der Politik, vom Staat kommend. So wie ich halt auch gesagt hatte, so Sprachangebote und Integrationsangebote mit Gruppen, wo sich Leute, die sich vielleicht auch integrieren wollen, aussprechen können und sonst auch Lehrangebote wie an Schulen und Universitäten, dass halt es denen leichter gemacht wird, sich zu integrieren und es auch Möglichkeiten gibt, an wen die sich wenden können " (M4: 22-27).

- „Ich find', es hat irgendwie zwei Seiten. Also einmal halt vom Staat, also dass genügend Angebote gestellt werden, weil's denen ja auch wichtig ist, dass sich die Personen integrieren, damit die Gesellschaft reibungslos funktionieren kann. Aber dann auch von der Person, die integriert werden soll, weil da gehört, finde ich, auch 'n gewisser Aspekt an Motivation und Wille dazu, weil sonst funktioniert das, denk' ich, nicht“" (M4: 30-35).

- „Oder auch 'ne gute Information für die zuziehenden Menschen, sodass sie überhaupt wissen, wohin sie sich wenden können und welche Möglichkeiten es gibt" (M2: 23-25). 


\subsubsection{Zusammenfassung und Diskussion der Ergebnisse der vertiefenden Interviews} mit Vereinsmitgliedern

Im Rahmen der Analyse der Interviews mit Vereinsvorständen konnten bereits angenommene Wirkweisen und Anforderungen in Bezug auf die Aspekte von Integration sowie Ausführungen $\mathrm{zu}$ Ausgängen und Adressatinnen und Adressaten identifiziert werden, mit Hilfe derer die quantitativen Daten vertieft werden können. Insgesamt zeigen sich bei der Analyse der explorativen und vertiefenden Interviews, wie bereits in der quantitativen Analyse, keine deutlichen Unterschiede zwischen Vereinsmitgliedern und Vorständen, weshalb die Interviews mit Vereinsmitgliedern der weiteren Ergänzung der Ausführungen der Vereinsvorstände dienen, indem beispielsweise hinterfragt wird, welche Werte im Rahmen der Kulturation übernommen werden sollten. Die vertiefenden Interviews dienten somit der weiteren Klärung einzelner Aspekte und auch der Ausgänge sowie der Adressatinnen und Adressaten von Integration.

Deutliche Unterschiede zwischen Personen mit und ohne Migrationshintergrund zeigen sich bei der Analyse der vertiefenden Interviews nicht. Lediglich das Sprechen über Integration scheint bei Personen mit Migrationshintergrund stark vor dem Hintergrund der eigenen Erfahrung reflektiert zu werden, während Integration für Personen ohne Migrationshintergrund eher als abstraktes Konstrukt wahrgenommen wird. Dieser Bezug auf die eigene Integrationserfahrung zeigte sich bereits bei Schramkowksi (2009) und führte, im Gegensatz zu den hier vorliegenden Interviews, zu einer negativen Konnotation des Integrationsbegriffs als Konzept der Anpassung. Somit ist anzunehmen, dass die eigene Erfahrung bei Personen mit Migrationshintergrund auch immer die Sichtweise auf dieses Konzept färbt.

Wie bei den Vereinsvorständen liegt auch bei den Interviews mit Vereinsmitgliedern der Fokus auf der Dimension der Interaktion. Auch hier ist anzunehmen, dass diese Dimension für die Interviewpartnerinnen und -partner diejenige darstellt, die am ehesten wahrnehmbar ist, da sie im Alltagshandeln relevant wird.

Insgesamt wird im Rahmen der Beschreibungen von einzelnen Aspekten von Integration deutlich, dass Integration nicht als ein einseitiger Prozess anzusehen ist, sondern vielmehr Anforderungen an zu Integrierende und die Personen des Aufnahmelandes gerichtet werden. Gleiches zeigte sich auch schon bei den Ausführungen $\mathrm{zu}$ Anforderungen bei den Vereinsvorständen. So zeigt sich im Rahmen der Dimension der Interaktion, dass die Anerkennung/Akzeptanz mit einer gewissen Offenheit auf beiden Seiten einhergeht und auch die Teilnahmebereitschaft an vorhandene Teilnahmechancen gekoppelt ist. In Bezug auf die 
Teilnahmebereitschaft wird in den Interviews mit Vereinsmitgliedern zusätzlich deutlich, dass vorhandenen Vorurteile oder Stereotype die Teilnahme erschweren. Denn selbst wenn eine starke Teilnahmebereitschaft vorhanden ist, kann diese nicht in Integration münden ,wenn auf der anderen Seite nur 'ne Mauer steht,... “ (M5: 29).

Diese beidseitige Bezugnahme spielt auch im Rahmen der Kulturation eine Rolle. Dort zeigt sich, ergänzend $\mathrm{zu}$ den Ausführungen der Vereinsvorstände, dass die Anerkennung/Internalisierung von Grundnormen nicht nur auf die Normen im Aufnahmeland bezogen sein muss und auch keine vollständige Übernahme dieser Werte eingefordert wird. Vielmehr spielt auch die Anerkennung von anderen Kulturen bzw. ein gewisses Interesse dafür in der aufnehmenden Gesellschaft eine Rolle und es sollte ein Austausch über unterschiedliche Sichtweisen entstehen, sofern diese kulturellen Elemente von den Personen aus anderen Kulturkreisen auch nach außen getragen werden.

Darüber hinaus konnte mit Hilfe der Interviews das Verständnis wichtiger Werte im Rahmen der Kulturation näher dargestellt werden. Betrachtet man die Ausführungen der Interviewpartnerinnen und -partner $\mathrm{zu}$ wichtigen Werten, zeigt sich, dass diese immer kontextabhängig gedacht werden und in Abgrenzung $\mathrm{zu}$ anderen als nicht-westlich bezeichneten Werten. Daraus ergibt sich ein bestimmtes Bild der Adressatinnen und Adressaten von Integration, die in den vertiefenden und explorativen Interviews zunächst nur als Personen mit einem anderen kulturellen Hintergrund oder Fluchthintergrund dargestellt wurden. In Bezug auf diese Adressatinnen und Adressaten zeigt sich in den vertiefenden Interviews somit, dass sich ein anderer kultureller Hintergrund auf Personen aus nicht westlichen Staaten zu beziehen scheint, bei denen darüber hinaus ein anderer Werterahmen angenommen wird. Demnach richtet sich die Anerkennung/Internalisierung von Grundnormen hauptsächlich an Personen aus anderen Kulturkreisen und nicht, beispielsweise, auf Personen aus Europa.

Als wichtige anzuerkennende Werte nennen die Interviewpartnerinnen und -partner Toleranz, Weltoffenheit, die Gleichberechtigung von Mann und Frau, Nächstenliebe, Akzeptanz von Homosexualität und weiteren in anderen Regionen tabuisierten Themen, Gleichheit, freiheitliches Leben und auch das Grundgesetz mit den darin befindlichen Werten.

Bei der Dimension der Identifikation zeigt sich ebenfalls eine beidseitige Perspektive auf Integration, die vor allem auf Interaktion beruht. Die weiteren Erkenntnisse zur Dimension der Identifikation zeigen eine starke Verknüpfung mit der Dimension der Interaktion. Dies macht erneut deutlich, dass einzelne Dimensionen und Aspekte nicht allein gedacht werden können, sondern in einem Zusammenhang zueinanderstehen. Eine Identifikation in Form eines 
Zugehörigkeitsgefühls kann nach Meinung der Interviewpartnerinnen und -partner nur entstehen, wenn Personen sich durch andere Personen respektiert und akzeptiert fühlen. Das Zugehörigkeitsgefühl wird darüber hinaus auch als ein individueller Faktor im Rahmen der Integration angesehen, welcher, selbst wenn Personen als integriert gesehen werden, nicht vorhanden sein muss.

Im Gegensatz dazu werden die Aspekte der Platzierung eher als mögliche förderliche Faktoren für Integration beschrieben. Bezüglich der Platzierung konnte im Rahmen der vertiefenden Interviews geklärt werden, auf wen sich die Aspekte innerhalb dieser Kategorie beziehen. Es zeigt sich, dass Platzierungsaspekte, wie Bildungsstand, finanzieller Hintergrund oder beruflicher Status, in erster Linie keine direkten Anforderungen an zu integrierende Personen darstellen. Vielmehr können diese Aspekte förderlich auf die Integration von Personen wirken. Die Interviewpartnerinnen und -partner beschreiben hier, dass beispielsweise ein höherer Bildungsstand die Integration in die Gesellschaft erleichtern kann. Gleichzeitig kann ein niedriger Bildungsstand jedoch auch hinderlich für die Integration sein. Diese Sichtweise auf die Dimension der Platzierung als eher nachgeordnete Dimension von Integration geht mit den bisherigen Erkenntnissen aus Arbeiten zum Integrationsverständnis einher. Bereits Lutter (2011) konnte in seiner Untersuchung eine durchgängige Fokussierung von Schülerinnen und Schülern auf eine sozial-kulturelle Perspektive nachweisen, die sich auch in dieser Arbeit zeigt. Bei Schramkowksi (2007) wurden äußere Maßstäbe zur Bewertung von Integration im Rahmen der Interviews mit Expertinnen und Experten sogar kritisch gesehen, da beispielsweise das Kriterium der Teilhabe am Arbeitsmarkt eine gewisse Relativität aufweist, weil dieser Aspekt im Falle einer plötzlichen Arbeitslosigkeit nicht mehr erfüllt wäre (Schramkowksi 2007: 148149). Gleichzeitig zeigen diese Ergebnisse zunächst Unterschiede zum Konzept von Esser (2001) auf, der die Platzierung als wichtigste Form der Integration in eine Gesellschaft beschreibt. Dennoch beschreibt auch Esser die Aspekte der Platzierung im Rahmen eines Marktgeschehens, in dem die Akteurinnen und Akteure durch Fertigkeiten und Ressourcen „Angebote“ machen, die von anderen Akteurinnen und Akteuren nachgefragt werden. Diese „Angebote“ werden auch im Rahmen der Interviews deutlich und dort mit den förderlichen oder hinderlichen Effekten für Integration verbunden. Die von Esser dargestellte enge Verbindung mit der Dimension der Kulturation zeigt sich jedoch nicht.

In der folgenden Abbildung 22 werden die Ausführungen der Vereinsvorstände und Vereinsmitglieder zu Aspekten und Dimensionen von Integration als Gesamtmodell dargestellt. Anforderungen werden erneut in blau und vermutete Wirkungen in gelb dargestellt. Die 
dargelegten Aspekte der Identifikation werden in grau dargestellt, da innerhalb dieser Dimension die Äußerungen der Interviewpartnerinnen und -partner keine konkreten Rückschlüsse auf Anforderungen und vermutete Wirkungen zulassen.

Abbildung 22: Zusammenführung der Ausführungen von Vereinsvorständen und Mitgliedern zu Dimensionen und Aspekten von Integration aus den Interviews

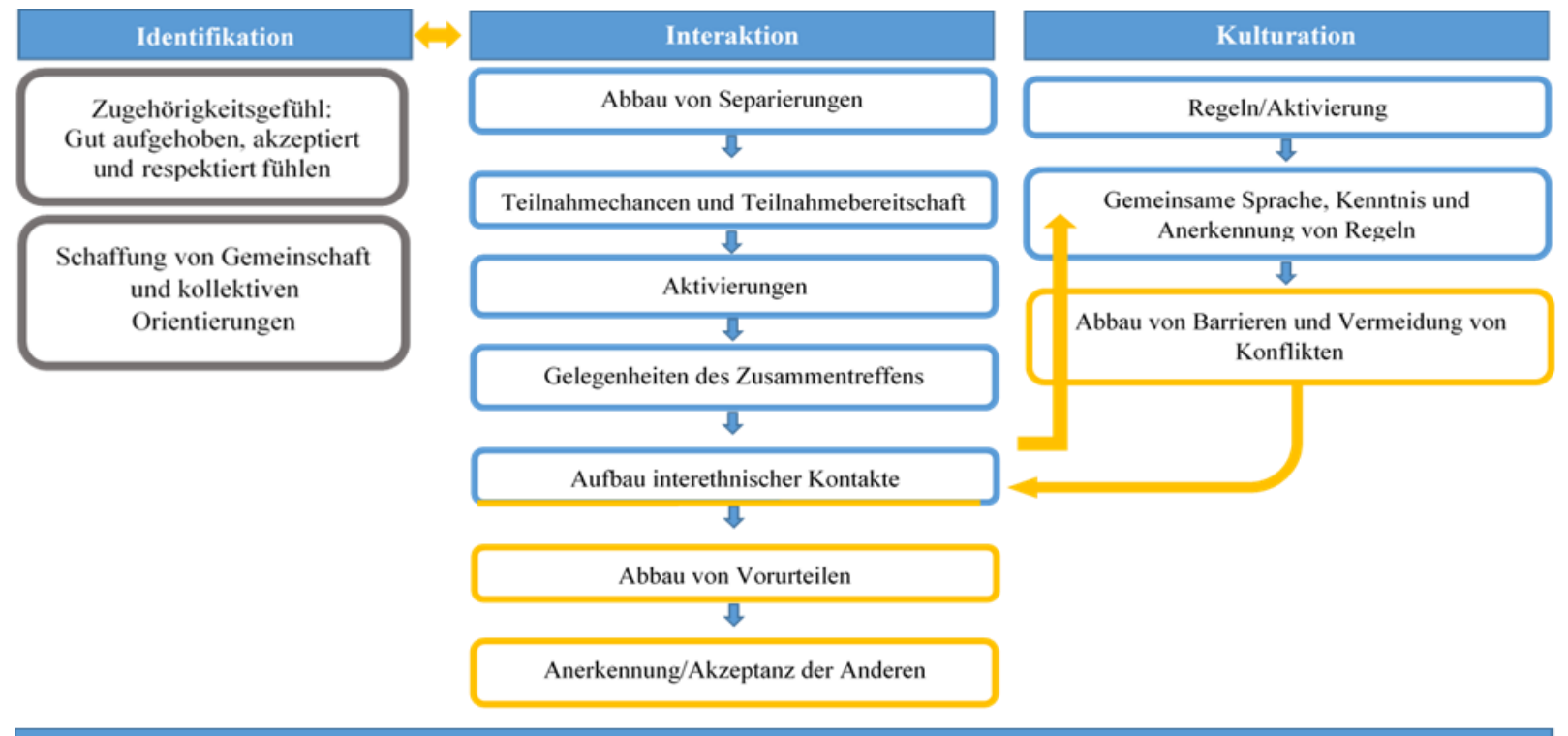

Platzierungsaspekte als förderliche Faktoren

Erläuterung: Blau = beschriebene Anforderungen; gelb = beschriebene Wirkweisen; grau = keine konkreten Rückschlüsse auf Anforderungen und vermutete Wirkungen durch Interviews

Quelle: Eigene Darstellung.

Insgesamt zeigen die Ausführungen, dass Integration überwiegend auf beidseitigen Prozessen beruht. Dies konnte auch bereits in den Vorstandsinterviews aufgezeigt und vor dem Hintergrund der theoretischen Entwicklung dargestellt werden. Denn in der theoretischen Debatte zeigte sich vor dem Hintergrund unterschiedlicher gesellschaftlicher und historischer Kontexte und der zunächst starken Dominanz des Assimilationsgedankens im chronologischen Verlauf ein Abkommen von der Idee, Integration wäre nur auf Leistung von Migrantinnen und Migranten begründet. Weiter geht diese Sichtweise mit den Ausführungen von Heckmann (2015) einher, der ein interaktionistisches Verständnis von Integration zugrunde legt und ausführt „Integration als Prozess der Mitgliedschaftswerdung und Angleichung der Lebensverhältnisse entwickelt sich schrittweise entlang der Dimensionen der strukturellen, kulturellen, sozialen und identifikativen Integration. Sie erfordert Integrationsleistungen der Migranten und bedarf der Offenheit und Förderung seitens der Aufnahmegesellschaft" (Heckmann 2015: 82). Darüber hinaus führt Heckmann jedoch auch aus, dass es sich zwar um einen wechselseitigen, jedoch nicht gleichgewichtigen Prozess handelt, da der Fokus der Anforderungen auf Seiten der Migrantinnen und Migranten liegt. Gleichzeitig handelt es sich 
um einen langen Prozess, der über Generationen hinweg verläuft (Heckmann 2015: 82). Diese zeitliche Perspektive und welche Personen die Hauptakteurinnen und -akteure im Rahmen der Integration sind, wird im Rahmen der Interviews nicht angesprochen.

Auch bezüglich der Ergebnisse der Integration lässt sich Integration als beidseitiger Prozess definieren. So zeigte sich bei den Interviews mit den Vereinsvorständen bereits eine Mischung aus pluralistischen und assimilativen Ansichten, die im Rahmen der Mitgliederinterviews weiter miteinander verknüpft werden können. Die Mitglieder beschreiben in den Interviews ein Bild von Integration, das eine Art Zusammenspiel von Anpassung und Akzeptanz darstellt. Eine Anpassung soll aus Sicht der Interviewpartnerinnen und -partner jedoch nur in bestimmten Bereichen stattfinden, während andere Differenzen akzeptiert werden. Dieses Zusammenspiel soll in einer Art Gleichheit münden, die sich auf einen gemeinsamen Werte- und Gesetzesrahmen sowie auf eine gemeinsame Sprache bezieht. In Bezug auf diese Aspekte existiert somit ein flexibler Prozess der gegenseitigen Anpassung und Akzeptanz, welcher über längere Zeit auch zu gesellschaftlichen Veränderungen, beispielsweise durch Anpassung des gemeinsamen Werterahmens, führen kann. Auch diese Ausführungen decken sich mit einem interaktionistischen Integrationsverständnis. Dieses ist nach Heckmann (1985) dann erreicht, wenn die aufnehmende Gesellschaft Elemente der zu integrierenden Kultur aufnimmt (Heckmann 1985: 23). 
6.4 Zusammenführung der Ergebnisse der unterschiedlichen Datentypen In einem nächsten Schritt sollen die Ergebnisse der quantitativen und qualitativen Analysen zusammengeführt werden. Dies erfolgt innerhalb von zwei Unterkapiteln. Das erste Unterkapitel umfasst die Ergebnisse zu Dimensionen und Aspekten von Integration und das zweite zu Ergebnissen sowie Adressatinnen und Adressaten von Integration.

\subsubsection{Dimensionen von Integration}

Im Rahmen der quantitativen Ergebnisse konnten zum einen signifikante Unterschiede zwischen den Einschätzungen zu Aspekten von Integration von Personen mit und ohne Migrationshintergrund sowie zwischen unterschiedlichen Migrationsgenerationen aufgezeigt werden. Zum anderen konnten die Dimensionen und Aspekte auch anhand ihrer Bedeutung für Integration geordnet werden. Die qualitativen Ergebnisse dienen hingegen dazu, Einschätzungen zu Anforderungen und Wirkweisen einzelner Dimensionen und Aspekte sowie die Ausgestaltung einzelner Aspekte zu analysieren.

Abbildung 16 (S. 141) zeigt die Ergebnisse der quantitativen Analyse, indem die Dimensionen sowie die darin befindlichen Aspekte, anhand der Bewertungen durch die Befragten, in ihrer Bedeutung für Integration geordnet wurden. Blau hervorgehoben sind dabei die Aspekte, bei denen sich signifikante Unterschiede zwischen Personen mit und ohne Migrationshintergrund zeigen.

Für die Befragten ist vor allem die Dimension der Interaktion von Bedeutung und innerhalb dieser Dimension der Wille und Chancen an der Gesellschaft teilzunehmen. Signifikante Unterschiede in der Bewertung durch Personen mit und ohne Migrationshintergrund zeigen sich bei den Aspekten Chancen zur Teilnahme an der Gesellschaft und interethnische Kontakte, welche beide durch Personen mit Migrationshintergrund höher bewertet werden. Auf die Interaktion folgen die Identifikation und Kulturation, im Rahmen derer sich keine Unterschiede zwischen Personen mit und ohne Migrationshintergrund feststellen lassen. Die Kulturation umfasst in der Befragung zwei Aspekte, von denen die Anerkennung wichtiger Werte als wichtiger bewertet wird als sprachliche Fähigkeiten. Insgesamt als am „unwichtigsten“ für Integration wird die Dimension der Platzierung beschrieben. Innerhalb dieser Dimension zeigen sich lediglich beim Aspekt der rechtlichen Gleichstellung signifikante Unterschiede zwischen Personen mit und ohne Migrationshintergrund. Dieser Aspekt stellt zusätzlich den am wichtigsten bewerteten Aspekt der Platzierung dar. Darauf folgt die Zufriedenheit mit der beruflichen Stellung, der Zugang zum Wohnungsmarkt, Bildungsstand und berufliche Stellung. 
Darüber hinaus konnten auch signifikante Unterschiede bei der Bewertung einzelner Aspekte zwischen unterschiedlichen Migrationsgenerationen und Personen mit einem europäischen und außereuropäischen Migrationshintergrund aufgezeigt werden. Hierbei spielt vor allem der Kontakt zwischen Personen verschiedener Kulturen eine Rolle. Dieser Aspekt scheint mit dem tatsächlichen vermehrten Vorhandensein interkultureller Kontakte von Personen mit Migrationshintergrund, der zweiten Generation von Migrantinnen und Migranten sowie Personen mit einem europäischen Migrationshintergrund einherzugehen, die diesen Aspekt jeweils als bedeutender für Integration bewerten.

Bringt man die im Rahmen der quantitativen Analysen vorgenommene Anordnung der Dimensionen in ihrer Bedeutung nun mit dem Modell zusammen, das im Rahmen der Analyse der Interviews entstanden ist (Abbildung 22), welches vor allem Anforderungen und Wirkweisen abbildet, zeigt sich folgendes Gesamtbild (Vgl. Abbildung 23).

Abbildung 23: Gesamtbild der subjektiven Sichtweisen auf Dimensionen und Aspekte von Integration aus Erhebung und Interviews

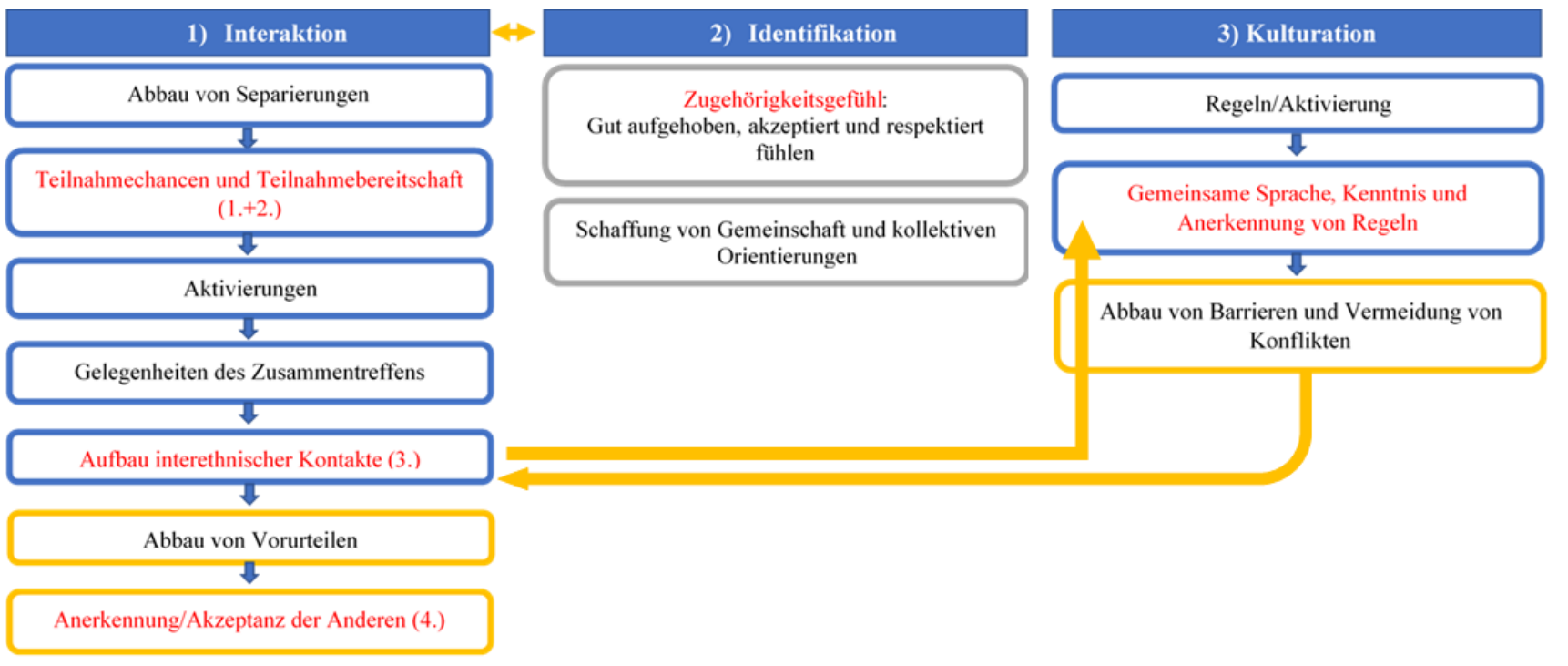

4) Pla tzierungsaspekte als förderliche Faktoren

Rechtliche Gleich stellung, Wohnen, Bildung, berufliche Position, Einkommen, Besetzung bestimmter Positionen und Teilhabe

Erläuterung: Blau = beschriebene Anforderungen; gelb = beschriebene Wirkweisen; rot = Aspekte wurden in der Online-Befragung zur Bewertung vorgelegt und fanden auch in den Interviews Erwähnung; rote Nummerierung: Stellung der ihnen zugemessenen Bedeutung für Integration innerhalb der Dimension der Interaktion

Quelle: Eigene Darstellung. 
Die Abbildung zeigt die beschriebenen Anforderungen erneut in blau und die Wirkweisen in gelb. Darüber hinaus sind die Aspekte, die sowohl in der Befragung zur Bewertung vorgelegt wurden als auch in den Interviews erwähnt wurden, in rot dargestellt. Die Nummerierungen der Aspekte der Interaktion geben ihre Stellung in der ihnen zugemessenen Bedeutung für Integration, innerhalb der Dimension der Interaktion, anhand der quantitativen Befragung wieder. Darüber hinaus sind die Dimensionen nach der ihnen zugemessenen Bedeutung angeordnet.

Es zeigt sich zunächst, dass alle im Fragebogen abgefragten Aspekte auch im Rahmen der Interviews relevant werden. Eine Ausnahme bildet nur die Zufriedenheit mit der sozialen und beruflichen Stellung. Außerdem konnten im Rahmen der Interviews zusätzliche Aspekte, die für die Ausgestaltung der einzelnen Dimensionen relevant sind, dargestellt werden, wodurch sich ein sehr differenziertes Bild der subjektiven Sichtweisen auf Integration ergibt. Durch die ergänzten Aspekte wird außerdem deutlich, dass Integration als ein beidseitiger Prozess gesehen wird, der sowohl Anforderungen an die zu Integrierenden als auch an die Gesellschaft stellt.

Zur Dimension der Interaktion wurden durch die Interviews mit Vereinsvorständen die Aspekte Abbau von Separierungen, Aktivierungen, Gelegenheiten des Zusammentreffens und der Abbau von Vorurteilen ergänzt. Insgesamt stehen diese Aspekte innerhalb der Dimension der Interaktion jedoch nicht einfach nebeneinander, vielmehr konnten erwartete Wirkweisen identifiziert werden. Demnach führt, nach Ansicht der Interviewpartnerinnen und -partner, ein Abbau von Separierungen erst dazu, dass Teilnahmechancen entstehen können. Diese Teilnahmechancen setzen gleichzeitig jedoch auch eine gewisse Teilnahmebereitschaft der zu integrierenden Personen voraus. Damit die daraus entstehenden Gelegenheiten des Zusammentreffens auch wahrgenommen werden, sind auch gewisse Aktivierungen auf Vereinsseite nötig. Die Vereine müssen somit im Prozess der Integration aktiv werden, damit diese gelingen kann. Entstandene Gelegenheiten des Zusammentreffens können dann wiederrum in den Aufbau interethnischer Kontakte münden, die den Abbau von Vorurteilen fördern und so zu einer Anerkennung und Akzeptanz der Anderen führen. Es zeigt sich somit im Rahmen der Interaktion, dass nicht nur die Aspekte aus den Integrationskonzepten eine Rolle für Integration spielen, sondern darüber hinaus auch aktive Maßnahmen nötig sind, die die Integration positiv unterstützen. Interessant im Ablauf der dargestellten Wirkweisen ist dabei, dass die im Fragebogen abgefragten Aspekte im Verlauf in der Reihenfolge beschrieben werden, wie sie auch in ihrer Bedeutung bewertet wurden. Als bedeutendster Aspekt der 
Interaktion wurden Teilnahmechancen und Teilnahmebereitschaft bewertet. Diese tauchen auch im Verlauf der Dimension zuerst auf, darauf folgen der Aufbau interethnischer Kontakte und die Akzeptanz/Anerkennung der Anderen, die auch absteigend nach der ihnen zugemessenen Bedeutung im Prozess dargestellt werden.

Die Dimension der Identifikation stellt in den quantitativen Analysen die zweitwichtigste Dimension dar. Im Rahmen der Interviews zeigen sich hier starke Verflechtungen mit der Dimension der Interaktion, da das Zugehörigkeitsgefühl als in einer gewissen Weise abhängig von der Interaktion mit anderen Personen dargestellt wird. Darüber hinaus konnte mit Hilfe der Interviews näher geklärt werden, was unter dem Vorhandensein eines Zugehörigkeitsgefühls verstanden wird.

Das Zugehörigkeitsgefühl wird von den Befragten als individueller Faktor beschrieben, das nicht vorhanden sein muss, wenn die Personen von anderen als integriert wahrgenommen werden. Dieses Zugehörigkeitsgefühl kann darüber hinaus nur in Interaktion mit anderen entstehen, indem sich die Personen durch andere Personen respektiert und akzeptiert fühlen.

Verflechtungen mit der Dimension der Interaktion zeigen sich auch bei der Kulturation. Diese Dimension ist in der Bewertung ihrer Bedeutung an dritter Stelle anzusiedeln. Auch hier konnten die im Fragebogen abgefragten Aspekte, wie die Anerkennung wichtiger Werte und sprachliche Fähigkeiten, durch die Interviews weiter ergänzt werden. Hier führen die Interviewpartnerinnen und -partner an, dass eine Voraussetzung für die Anerkennung wichtiger Werte und auch für sprachliche Fähigkeiten zunächst das Aufstellen von Regeln und auch Aktivierungen sind. Darüber hinaus führt die Anerkennung wichtiger Werte und eine gemeinsame Sprache dann zum Abbau von Barrieren und der Vermeidung von Konflikten. Dieser Abbau von Barrieren kann dann zum Aufbau interethnischer Kontakte, innerhalb der Dimension der Interaktion, führen. Gleichzeitig kann der Aufbau interethnischer Kontakte jedoch auch die Anerkennung wichtiger Werte sowie sprachliche Fähigkeiten fördern.

Neben der Ergänzung von den im Fragebogen enthaltenen Aspekten war es im Rahmen der Interviewanalyse auch möglich, einzelne, eher abstrakt gefasste Aspekte näher zu klären. Diese Klärung umfasst den Aspekt der Anerkennung wichtiger Werte.

Als wichtige Werte werden von den Interviewpartnerinnen und -partnern Toleranz, Weltoffenheit, die Gleichberechtigung von Mann und Frau, Nächstenliebe, Akzeptanz von Homosexualität und weiteren in anderen Regionen tabuisierten Themen, Gleichheit, freiheitliches Leben und auch das Grundgesetz mit den darin befindlichen Werten genannt. 
Dabei zeigt sich auch, dass die Interviewpartnerinnen und -partner häufiger von „westlichen Werten“" sprechen. Demnach bezieht sich dieser Aspekt der Integration vor allem auf Personen mit einem anderen kulturellen Hintergrund oder Fluchthintergrund, bei denen ein anders ausgestalteter Werterahmen angenommen wird. Personen aus Nachbarstaaten oder europäischen Staaten werden somit im Rahmen dieses Aspekts nicht als Adressatinnen und Adressaten wahrgenommen.

Die Ausführungen zeigen bereits, dass sich starke Verflechtungen zwischen den Dimensionen der Interaktion, Identifikation und Kulturation ergeben. Im Gegensatz dazu wird die Dimension der Platzierung, die auch im Rahmen der Bewertung der Bedeutung für Integration an letzter Stelle steht, eher als eine Dimension gesehen, die förderlich auf Integration wirken kann. Dabei spielen vor allem individuelle Faktoren der zu integrierenden Personen eine Rolle. Diese Faktoren umfassen Wohnen, Bildung, berufliche Position, Einkommen sowie die Besetzung bestimmter Positionen und Teilhabe. Gleichzeitig wird jedoch auch die rechtliche Gleichstellung als wichtigster Aspekt der Platzierung gesehen. Demnach zeigt sich nicht wie bei den anderen Dimensionen ein Zusammenspiel von Opportunitäten, Leistungen der Gesellschaft und Leistungen der zu integrierenden Personen, weshalb die Platzierung hier als eine Art Basis dargestellt wird, die zwar förderlich wirken kann, aber nicht muss.

\subsubsection{Ergebnisse sowie Adressatinnen und Adressaten von Integration}

Die Analysen zu Ergebnissen von Integration zeigen klare Überschneidungen zwischen den quantitativen und qualitativen Ergebnissen. Darüber hinaus können die quantitativen Ergebnisse zu Zusammenhängen zwischen der Zustimmung zu Integrationskonzepten und sozialstrukturellen Merkmalen sowie Merkmalen der Sportvereine ergänzend herangezogen werden.

Im Rahmen der quantitativen Analysen konnte herausgestellt werden, dass sich signifikante Unterschiede zwischen Personen mit und ohne Migrationshintergrund nur in Bezug auf das Item „Es wäre gut, wenn alle Zuwanderer in Deutschland ihre Kulturen beibehielten“ zeigen. Dieses wird von Personen mit Migrationshintergrund höher und damit als wünschenswerter bewertet. Beim Vergleich von Vereinsvorständen und Mitgliedern zeigen sich bei den Ausgängen von Integration hingegen keine signifikanten Unterschiede.

Insgesamt wird vor allem der Ausgang der Mehrfachintegration von den Befragten favorisiert. Diese wurde im Fragebogen mit Hilfe des Statements „Zuwanderer sollten sowohl in ihre 
Herkunftsgesellschaft als auch in die deutsche Gesellschaft eingebunden sein" bewertet. Gleichzeitig zeigte sich aber auch, dass assimilative Tendenzen in Form der Aussage „Menschen, die nach Deutschland kommen, sollten ihr Verhalten der deutschen Kultur anpassen“ die zweithöchste Bewertung aufweisen, während eher Unsicherheit darüber besteht, ob Zugewanderte ihre Kultur beibehalten sollten, da im Rahmen der Bewertung dieses Items oft die Mittelkategorie angegeben wurde. Die Segregation im Rahmen derer Zugewanderte unter sich bleiben sollen, wird hingegen überwiegend abgelehnt. Diese Mischung aus einer Favorisierung der Mehrfachintegration und assimilativen Tendenzen sowie einer vorhandenen Unsicherheit über die Beibehaltung der Kultur von Zugewanderten, kann durch die explorativen und vertiefenden Interviews näher verknüpft werden.

Zunächst zeigte sich auch in den explorativen Interviews mit Vereinsvorständen eine Mischung aus pluralistischen und assimilativen Tendenzen. Pluralistische Ansichten umfassten dabei vor allem eine Ermöglichung des Neben- oder Miteinanders unter der Voraussetzung des gegenseitigen Respekts. Diese Ansichten wurden jedoch überwiegend in der Beschreibung von Leitlinien der Vereine im Sinne von passiven normativen Zielvorstellungen deutlich, während assimilative Tendenzen in der Beschreibung von Beispielen gelungener Integration dargestellt wurden. Dies lässt eine Diskrepanz zwischen wünschenswerten Ergebnissen von Integration, in Form der pluralistischen Ansichten und assimilativen Tendenzen, als Wahrnehmung von Integrationsverläufen erkennen. Zusätzlich beschreiben die Vereinsmitglieder in den Interviews die Ergebnisse von Integration als eine Art Zusammenspiel von Anpassung und Akzeptanz, welches in einer gewissen Gleichheit im Rahmen eines gemeinsamen Werte- und Gesetzesrahmens sowie einer gemeinsamen Sprache enden soll. Die Interviewpartnerinnen und -partner beschreiben, dass Anpassung somit nur in bestimmten Bereichen stattfindet, während Differenzen in anderen Bereichen akzeptiert werden. Diese Ausführungen zeigen die Verflechtung zwischen Pluralismus und assimilativen Tendenzen. Zwar sollte in gewissen Bereichen eine Anpassung stattfinden, dies umfasst aber nicht die Aufgabe der eigenen Kultur, sondern nur die Schaffung von Gemeinsamkeiten oder Gleichheit in bestimmten Bereichen. Im Sinne dessen wird die eigene Kultur beibehalten, während trotzdem auch Elemente der Aufnahmegesellschaft an Bedeutung gewinnen. In den quantitativen Analysen zeigte sich, anders als in den Interviews, ein signifikanter negativer Zusammenhang zwischen der Befürwortung der Konzepte Pluralismus und Assimilation. Darüber hinaus konnten signifikante Zusammenhänge zwischen diesen Konzepten und Aspekten von Integration aufgezeigt werden. Die Zusammenhänge zwischen den beiden Konzepten und Aspekten von Integration weisen dabei einige Unterschiede auf, die die Schlussfolgerung zulassen, dass 
Assimilation und Pluralismus nebeneinander bestehen können, da sie unterschiedliche Bereiche von Integration adressieren. Für die Assimilation relevant sind demnach vor allem gute Deutschkenntnisse, die Anerkennung wichtiger Werte, der Wille an der Gesellschaft teilzunehmen sowie der Bildungsstand. Im Gegensatz dazu zeigen sich signifikante negative Zusammenhänge mit den Chancen an der Gesellschaft teilzunehmen und den Kontakten zwischen Menschen unterschiedlicher Kulturen. Demnach werden diese Aspekte bei einer Befürwortung der Assimilation als weniger wichtig für Integration angesehen. Die Befürwortung des Items zu Pluralismus geht hingegen mit einer geringeren Bedeutung der Anerkennung wichtiger Werte einher, während die Aspekte Kontakte zwischen Menschen unterschiedlicher Kulturen, Vorhandensein eines Zugehörigkeitsgefühls, gleiche Rechten und Zugang zum Wohnungsmarkt eine höhere Relevanz beigemessen wird.

Mit Hilfe der quantitativen Daten konnten außerdem Zusammenhänge zwischen der Zustimmung $\mathrm{zu}$ den Integrationskonzepten und sozialstrukturellen Merkmalen sowie Merkmalen der Sportvereine analysiert werden, die die bisherigen Ausführungen ergänzen. Dazu wurden die folgenden vier Hypothesen aufstellt:

- H1: Das Alter der Befragten weist einen negativen Zusammenhang mit der Unterstützung pluralistischer Konzepte auf.

- H2: Personen mit Migrationshintergrund unterstützen pluralistische Konzepte stärker als andere Konzepte.

- H3: Umso höher der Anteil von Personen mit Migrationshintergrund in der Trainingsgruppe, desto stärker werden pluralistische Konzepte unterstützt.

- H4: Umso höher der Bildungsstand, desto stärker werden pluralistische Konzepte unterstützt.

Im Rahmen der Analyse konnten die Hypothesen H2 und H3 bestätigt werden. In Bezug auf H1 und H4 zeigte sich kein bzw. nicht die erwartete Richtung des Zusammenhangs mit der Aussage zum pluralistischen Ausgang von Integration. Dafür konnte festgestellt werden, dass die Zustimmung zur Mehrfachintegration mit steigendem Alter sinkt, während die Befürwortung der Segregation steigt. Dies zeigt, dass mit steigendem Alter eher eine Abgrenzung zwischen einheimischen Personen und Zugewanderten, die ihre Kultur beibehalten wollen, favorisiert wird. Beim Bildungsstand zeigten sich negative Zusammenhänge mit dem Ausgang des Pluralismus und der Segregation. Demnach wird mit steigendem Bildungsstand eine vollständige Beibehaltung der Kultur weniger unterstützt, entscheiden sich die 
Zugewanderten jedoch für die Beibehaltung ihrer Kultur, sollte es auch nicht zur Segregation kommen.

Neben den Ausgängen von Integration konnten auch die Adressatinnen und Adressaten von Integration mit Hilfe der explorativen und vertiefenden Interviews analysiert werden.

Sowohl in den Interviews mit Vereinsvorständen als auch mit Mitgliedern zeigt sich, dass nicht nur Personen mit Migrationshintergrund als Adressatinnen und Adressaten für Integration angesehen werden. Dabei kann zunächst zwischen als zu integrierend Wahrgenommenen und Personen, die etwas für Integration leisten müssen, unterschieden werden. Die Vereinsvorstände nennen als zu integrierende Personen vor allem Personen mit einem anderen kulturellen oder Fluchthintergrund. Darüber hinaus nennen die Vereinsvorstände jedoch auch Eigenschaften wie Alter, Geschlecht, Behinderung und soziale Lage. Eine solche Erweiterung der Adressatinnen und Adressaten zeigt sich auch in den Interviews mit den Vereinsmitgliedern. Diese beziehen neben Personen mit einem anderen kulturellen oder Fluchthintergrund ebenfalls Personen mit Behinderung oder gesundheitlichen Einschränkungen sowie Minderheiten ein. Demnach wird in der subjektiven Wahrnehmung von Integration deutlich, dass das Konzept nicht nur auf Personen mit Migrationshintergrund bezogen wird. Bezüglich der Personen mit einem anderen kulturellen Hintergrund konnte im Rahmen der Mitgliederinterviews herausgearbeitet werden, dass dieser Personenkreis auf eine bestimmte Herkunft beschränkt wird. Denn die Interviewpartnerinnen und -partner beschreiben vor allem Personen aus nicht westlichen Staaten als Adressatinnen und Adressaten von Integration, bei denen zudem ein anderer Werterahmen angenommen wird.

Neben diesen als zu integrierend wahrgenommenen Personen spielen aber auch Personen eine Rolle, die etwas für Integration leisten sollen. Die Vereinsvorstände beziehen sich dabei auf „Einheimische“ und Zuständige bzw. Ansprechpartnerinnen und -partner im Verein. Dabei kommt vor allem den Ansprechpersonen im Verein eine steuernde Funktion im Integrationsprozess zu, in der vor allem die Vermittlung zwischen den zu integrierenden Personen und den „Einheimischen“ im Mittelpunkt steht. Integration bedarf somit bestimmter Aktivierungen und Maßnahmen sowie einer gewissen Begleitung und findet nicht automatisch statt.

Auch bei den Vereinsmitgliedern spielen „Einheimische“, die in diesen Interviews als Personen beschrieben werden, die bereits Teil der Gesellschaft sind, eine Rolle im Rahmen der Integration. Darüber hinaus werden auch politische Maßnahmen angesprochen. Auch hier werden somit wieder aktive Maßnahmen, wie Sprachkurse, die Bereitstellung von 
Informationen und Unterstützungsangebote, erläutert. Somit sind aktive Maßnahmen zur Förderung von Integration nicht nur innerhalb von Sportvereinen nötig, wie von den Vereinsvorständen dargestellt, sondern auch auf gesamtgesellschaftlicher Ebene. 


\section{Diskussion und Fazit}

Im Rahmen der vorliegenden Arbeit sollte der Begriff der Integration und damit verbundene Zielvorstellungen aus Sicht der Akteurinnen und Akteure in Sportorganisationen beleuchtet werden. Dazu wurde eine Studie im Mixed-Methods-Design durchgeführt, die eine fragebogenbasierte Umfrage, explorative Interviews mit Vereinsvorständen und vertiefende Leitfadeninterviews mit Vereinsmitgliedern umfasste. Fragen, die bezüglich der Sichtweisen der Akteurinnen und Akteure aufgeworfen wurden, sind:

- Welche subjektiven Sichtweisen von Integration (in Form von Verständnis von Integration und Einstellungen zu Integrationskonzepten/Ausgängen von Integration) haben die Akteurinnen und Akteure?

- Welche Dimensionen und Aspekte von Integration sind ihnen dabei wichtig?

- Welche Ergebnisse von Integration werden für wünschenswert gehalten?

- Wie hängen Ergebnisse und Aspekte von Integration zusammen?

- Inwiefern variieren die Sichtweisen auf Integration in Abhängigkeit von sozialstrukturellen Merkmalen und der Zusammensetzung der Mitglieder der Sportvereine?

In Bezug auf das subjektive Verständnis von Integration zeigt sich in den Ergebnissen zu Dimensionen und Aspekten sowie der Ausgänge von Integration ein starker Fokus auf Integration als beidseitiger Prozess. Dieser beidseitige Prozess geht mit einem interaktionistischen Ansatz einher, der somit als grundlegendes Verständnis von Integration herausgestellt werden kann.

Im Rahmen des interaktionistischen Ansatzes sind nicht nur Integrationsleistung der Migrantinnen und Migranten, sondern auch Offenheit und Förderung seitens der Aufnahmegesellschaft relevant (Heckmann 2015: 82). Diese Bedeutung der Aufnahmegesellschaft zeigt sich zum einen in der Bewertung im Fragebogen, in der auch Opportunitäten sowie Leistungen der Gesellschaft als wichtig bewertet wurden, sowie in den Interviews, in denen immer wieder auf Aufgaben der Gesellschaft verwiesen wurde. Diese Ausgestaltung von Integration als beidseitiger Prozess zeigt sich auch in den im theoretischen Rahmen behandelten Konzepten von Hoffmann-Nowotny (1973), Esser (2001), Heckmann et al. (2000) und im Bielefelder Desintegrationsansatz von Anhut und Heitmeyer (2009). Im Gegensatz dazu zeigt sich bei Eisenstadt (1952a) eher eine Fokussierung auf das Konzept der Assimilation und im Rahmen der genannten Aspekte von Integration eine Betonung der Leistung von Migrantinnen und Migranten, obwohl auch die Bereitschaft der 
Aufnahmegesellschaft für eine erfolgreiche Anpassung angesprochen wird. Die Ergebnisse zeigen, dass auch in der Wahrnehmung der Befragten Integration nicht als alleinige Leistung von Personen mit Migrationshintergrund anzusehen ist, sondern vielfältige Akteurinnen und Akteure in diesem Prozess aktiv werden müssen. Heckmann führt im Sinne des interaktionistischen Verständnisses jedoch auch aus, dass es sich zwar um einen wechselseitigen, jedoch nicht gleichwertigen Prozess handelt, da der Fokus der Anforderungen auf Seiten der Migrantinnen und Migranten liegt (Heckmann 2015: 82). Betrachtet man die in den Interviews hervorgebrachten Anforderungen, lässt sich dies zunächst nicht bestätigen und die Adressierung unterschiedlicher Akteurinnen und Akteure scheint eher ausgewogen. Auffällig ist hierbei, dass die durch die Vereinsvorstände zusätzlich genannten Aspekte der einzelnen Dimensionen hauptsächlich Anforderungen an die Gesellschaft bzw. Zuständige im Verein beinhalten. So nannten die Vorstände im Rahmen der Interaktion zusätzlich den Abbau von Separierungen und Aktivierungen als Anforderung der Interaktion sowie das Vorhandensein von Regeln und Aktivierungen für die Dimension der Kulturation.

Der Einbezug unterschiedlicher Akteurinnen und Akteure wird im Rahmen der Interviews auch in der Darstellung der Adressatinnen und Adressaten deutlich. Hier verwiesen sowohl die Vereinsvorstände als auch die Mitglieder auf unterschiedliche Akteurinnen und Akteure, die sich zunächst in als zu integrierend Wahrgenommene und Handelnde unterscheiden lassen. Als $\mathrm{zu}$ integrierend werden in den Interviews mehrere Personengruppen angesprochen. Diese umfassen Personen mit Migrations- oder Fluchthintergrund, Personen mit Behinderung oder gesundheitlichen Einschränkungen, Personen, die in eine Gruppe aufgenommen werden, Minderheiten sowie die Differenzkategorien Alter, Geschlecht und soziale Lage. Dabei liegt der Fokus zwar, wie bei den vorgestellten Integrationskonzepten, auf Personen mit Migrationshintergrund. Die weitere Fülle an Differenzkategorien zeigt aber eher eine Vergleichbarkeit mit dem Konzept des Diversitätsmanagements und damit einhergehenden Diversitätskategorien, im Rahmen derer viele Personengruppen miteinbezogen werden. Die Kernkategorien von Diversität beziehen sich in diesem Konzept auf Geschlecht, Alter, Behinderung, Nationalität, Religion/ Weltanschauung sowie sexuelle Orientierung und können darüber hinaus durch weitere Kategorien, die die Partizipation von Individuen betreffen, erweitert werden (Rulofs 2014: 8/9).

Im Gegensatz zu einigen der vorgestellten Integrationskonzepten, die hauptsächlich Migrantinnen und Migranten mit eigener Migrationserfahrung als zu integrierende Personen behandeln, ist die Gruppe der Adressatinnen und Adressaten innerhalb der subjektiven 
Sichtweisen somit vielfältiger, womit eine angenommene Übertragbarkeit des Konzepts auf weitere Personengruppen einhergeht. Diese Übertragbarkeit zeigt sich auch bereits in den Ausführungen von Hoffmann-Nowotny (1973), der ausführt, dass sich Integration und Assimilation nicht nur auf Migrantinnen und Migranten, sondern auch auf andere Gruppen innerhalb der Gesellschaft beziehen können (Hoffmann-Nowotny 1973: 172). Im Gegensatz dazu fokussiert beispielsweise das Konzept von Esser (2001) mit der deutlichen Orientierung am Einbezug in die Herkunfts- oder/und Aufnahmegesellschaft lediglich die Gruppe der Migrantinnen und Migranten mit eigener Migrationserfahrung.

Personen, die als handelnd in Bezug auf Integration in den Interviews Erwähnung finden, sind „Einheimische“ bzw. Personen, die bereits Teil der Gesellschaft sind, die Politik und in Bezug auf den Sportverein spezifische Ansprechpersonen oder Zuständige. Diese Personengruppen gehen mit der Darstellung in den theoretischen Konzepten einher, in denen Opportunitäten sowie Aufgaben der Gesellschaft sichtbar wurden.

Darüber hinaus ergibt sich im Rahmen der Interviews eine genauere Darstellung der Personen mit Migrations- oder Fluchthintergrund. Während mit dieser Personengruppe in den vorgestellten Integrationskonzepten Personen mit eigener Migrationserfahrung gemeint sind, umfasst diese Gruppe aus Sicht der Interviewpartnerinnen und -partner auch Personen ohne eigene Migrationserfahrung. Zusätzlich fokussiert dieser Begriff aber hauptsächlich Personen aus nicht westlichen Staaten, da bei diesen Personen ein anderer Werterahmen angenommen wird.

In Bezug auf die Frage, welche Dimensionen und Aspekte von den Akteurinnen und Akteuren als besonders wichtig für Integration bewertet werden, zeigt sich, dass vor allem der Dimension der Interaktion große Bedeutung zukommt. Diese Dimension konnte im Rahmen der Befragung als wichtigste Dimension herausgestellt werden und auch in den Interviews fand sich ein starker Fokus auf diese Dimension.

Die Bewertung der Interaktion als wichtigste Dimension der Integration weicht von den Ausführungen von Esser (2001) ab, der die Dimension der Platzierung als die bedeutendste ansieht und ausführt: „Die Plazierung auf den (möglichst auch: zentralen) Positionen einer Gesellschaft ist der Schlüssel für jede nachhaltige Sozialintegration“ (Esser 2001: 10). Gleichzeitig findet die Platzierung bei Esser in den nicht-formellen Bereichen der Gesellschaft aber erst über die Interaktion statt. Darüber hinaus beschreibt Esser die Aspekte der Platzierung im Rahmen eines Marktgeschehens, in dem die Akteurinnen und Akteure durch Fertigkeiten und Ressourcen „Angebote“ machen, die von anderen Akteurinnen und Akteuren nachgefragt 
werden. Diese „Angebote“ werden auch im Rahmen der Interviews deutlich und dort mit den förderlichen oder hinderlichen Effekten für Integration verbunden. Aspekte der Platzierung können, im Rahmen der subjektiven Sichtweisen auf Integration, diese somit fördern oder hindern, spielen insgesamt aber eher eine untergeordnete Rolle.

Die Betonung der Platzierung zeigt sich auch bei Hoffmann-Nowotny (1973) der ausführt, dass im Rahmen der Integration zunächst von Bedeutung ist, ob die Gesellschaft „die zentralen Statuslinien für die Migranten öffnet oder sie weitgehend geschlossen hält" (HoffmannNowotny 1973: 172). Im Gegensatz dazu beschreibt Eisenstadt (1952a), dass es für eine erfolgreiche Absorption in die Aufnahmegesellschaft zu einer Expansion der sozialen Interaktionen und Partizipation kommen muss, wonach in diesem Konzept vor allem Interaktionen an Relevanz gewinnen (Eisenstadt 1952: 223). Gleiches zeigt sich auch beim Ansatz von Anhut und Heitmeyer (2009), im Rahmen dessen es vor allem um das Erreichen von Anerkennung geht. Im Rahmen der vorgestellten Integrationskonzepte liegen somit unterschiedliche Sichtweisen bezüglich der Bedeutung der unterschiedlichen Dimensionen vor, die sich vor allem durch eine Fokussierung auf Interaktion oder auf Platzierung unterscheiden. Die im Forschungsstand vorgestellte Arbeit von Lutter (2011) zeigt, dass bei den untersuchten subjektiven Sichtweisen von Schülerinnen und Schülern vor allem eine sozial-kulturelle Perspektive fokussiert wird, während strukturelle Aspekte kaum angesprochen wurden. Darüber hinaus zeigte sich bei Schramkowksi (2007) im Rahmen der Interviews mit Expertinnen und Experten ein kritischer Umgang mit Aspekten der Platzierung, da diese eine gewisse Relativität aufweisen und beispielsweise der Verlust des Arbeitsplatzes dazu führt, dass Aspekte der Integration nicht mehr erfüllt wären (Schramkowski 2007: 148-149). Wie in der vorliegenden Arbeit dargestellt, scheint im Rahmen von subjektiven Sichtweisen somit der Dimension der Interaktion die größte Bedeutung zuzukommen. Dies steht somit konträr zu Ergebnissen der Integrationsforschung, in denen Aspekte der Platzierung, wie Bildung und berufliche Stellung sowie der Aspekt der Sprachkenntnisse aus der Dimension der Kulturation betont wird, da gerade diese Aspekte in der vorliegenden Arbeit als am wenigsten wichtig für Integration bewertet wurden. Zusammenhängen könnte dies damit, dass die Dimension der Interaktion diejenige ist, die die Befragten am ehesten wahrnehmen können, weil sie innerhalb derer selbst als Akteurin oder Akteur relevant werden, auch wenn sie keinen Migrationshintergrund besitzen. Aspekte wie der Bildungsstand oder die berufliche Stellung werden erst im späteren Verlauf von Interaktionen bekannt, da sie nicht direkt sichtbar sind. In Bezug auf die Sprachkenntnisse könnte eine gewisse Spezifik der Sportvereinsmitglieder 
vorliegen, da die Regeln der Sportarten, gerade wenn es sich um populären Sport handelt, oft bekannt sind und eine nonverbale Kommunikation ausreichen kann.

Neben der unterschiedlichen Bedeutung der Dimensionen konnten in den Interviews auch Verbindungen zwischen einzelnen Dimensionen aufgezeigt werden. Diese umfassen zum einen eine Verbindung zwischen der Interaktion und Identifikation, da nach Ansicht der Interviewpartner Identifikation nur in Interaktionen entstehen kann. Zum anderen zeigen sich Verbindungen zwischen einzelnen Aspekten der Interaktion sowie zwischen den Dimensionen der Interaktion und der Kulturation.

So beschreiben die Vereinsvorstände, dass Gelegenheiten des Zusammentreffens in den Aufbau interethnischer Kontakte münden können, die den Abbau von Vorurteilen fördern und so zu einer Anerkennung und Akzeptanz der Anderen führen. Diese beschriebene Wirkweise, zeigt, im Rahmen der Dimension der Interaktion, Übereinstimmungen mit der Kontakthypothese von Allport (1954) auf. Innerhalb der Kontakthypothese geht Allport davon aus, dass Vorurteile gegenüber anderen Personen vor allem auf deren nationale oder ethnische Zugehörigkeit zurückzuführen sind (Allport 1954: 34). Dies führt zu einer Differenzierung zwischen „Ingroups“ und „Outgroups“ (Allport 1954: 29-34). Die Problematik der Abgrenzung zwischen „Ingroups“ und „Outgroups“ sowie die vorhandenen Vorurteile können nach Allport jedoch überwunden werden, wenn häufiger Kontakt zwischen den Mitgliedern der unterschiedlichen Gruppen besteht (ebd.: S. 263-264). Der Abbau von Vorurteilen durch Kontakt kommt zustande, weil Vorurteile aus Unwissenheit über die andere Gruppe entstehen, die durch Kontakt und Interaktion abgebaut werden kann (Pettigrew 1986: 173). Zu Kontakt und einem Abbau der Vorurteile kommt es nach Allport vor allem dann, wenn gemeinsame Ziele vorliegen, die durch Kooperation erreicht werden können, ein gleicher Status vorhanden ist und der Kontakt zwischen den Gruppen durch soziale Normen unterstützt wird (Allport 1954: 281).

Darüber hinaus beschreiben die Vereinsvorstände, dass interethnische Beziehungen eine gemeinsame Sprache und Wertorientierung fördern, gleichzeitig aber auch der Abbau von Barrieren und die Vermeidung von Konflikten, die aus einer gemeinsamen Sprache und Wertorientierung hervorgehen, den Aufbau interethnischer Kontakte begünstigen können. Diese Verbindung von kulturellen Fertigkeiten und dem Aufbau interethnischer Kontakte stellt auch Esser (2001) heraus. Insgesamt geht er jedoch von einer sich selbst verstärkenden Beziehung von Kulturation, Interaktion und auch Platzierung aus. Diese kann vor allem bei starken ethnischen Segregationen, beispielsweise in Wohnvierteln, zu einer Spirale der sozialen Segmentation und der Entwicklung eigenethnischer Gemeinden führen (Esser 2001: 11). Den 
umgekehrten Fall, wenn interethnische Kontakte vorliegen, stellt Esser in seinen Ausführungen nicht dar. Jedoch ist anzunehmen, dass sich in diesem Fall Kulturation, Interaktion und Platzierung auch verstärken. Demnach lassen sich die Annahmen der Interviewpartnerinnen und -partner zur Verbindung von Kulturation und Interaktion ebenfalls als gegenseitige Verstärkung dieser Dimension ansehen. Die Platzierung spielt dabei für die Interviewpartnerinnen und -partner jedoch keine Rolle.

In Bezug auf wünschenswerte Ergebnisse von Integration kann festgestellt werden, dass der Assimilationsgedanke trotz der Wahrnehmung von Integration als beidseitiger Prozess nicht ganz an Bedeutung verliert. Sowohl in der quantitativen Befragung als auch in den Interviews zeigt sich eine Mischung aus pluralistischen und assimilativen Ansichten bezüglich der Ergebnisse von Integration. Dies macht deutlich, dass die breit geführte Diskussion um Assimilation oder Pluralismus eventuell auch nicht zu lösen ist und die beiden Konzepte, zumindest in der subjektiven Wahrnehmung der Befragten, nebeneinander bestehen können. Die Mischung aus pluralistischen und assimilativen Ansichten zeigte sich auch bereits in den Untersuchungen von Strahle und Baur (2009) sowie Baur und Mutz (2009). Mit Hilfe der vertiefenden Interviews konnte geklärt werden, wie diese Verbindung der beiden Konzepte gedacht wird. Im Rahmen der Interviews zeigten sich Beschreibungen, die eine Art Zusammenspiel von Anpassung und Akzeptanz darstellen. Die Anpassung soll jedoch nicht alle Bereiche übergreifen, sondern nur in bestimmten Bereichen stattfinden, während die Differenzen in anderen Bereich bestehen bleiben und akzeptiert werden. Dies lässt sich auch im Rahmen der Analysen zu den Zusammenhängen zwischen Ergebnissen und Aspekten von Integration abbilden, im Rahmen derer die Befürwortung dieser Konzepte mit einer gesteigerten Bedeutung unterschiedlicher Aspekte von Integration einherging. Die Interviewpartnerinnen und -partner beschrieben vor allem Anpassungsmechanismen zur Herstellung eines gemeinsamen Werte- und Gesetzesrahmens sowie einer gemeinsamen Sprache. In Bezug auf diese Aspekte existiert somit ein flexibler Prozess der gegenseitigen Anpassung und Akzeptanz, welcher über längere Zeit auch zu gesellschaftlichen Veränderungen, beispielsweise durch Anpassung des gemeinsamen Werterahmens, führen kann. Auch diese Ausführungen decken sich mit den Ausführungen von Heckmann (1985) zur interaktionistischen Integration, die erreicht ist, wenn die aufnehmende Gesellschaft Elemente der zu integrierenden Kultur aufnimmt (Heckmann 1985: 23). In einer späteren Arbeit betont Heckmann, dass es dabei nicht nur zu Veränderungen auf Seiten der Migrantinnen und Migranten kommt, sondern auch Veränderungen in der aufnehmenden Gesellschaft stattfinden, indem ethnische Grenzen aufgeweicht werden (Heckmann 1997: 4). 
Somit kann in Bezug auf die Ergebnisse von Integration festgehalten werden, dass die subjektiven Sichtweisen auf Integration einem interaktionistischen Verständnis am nächsten kommen, das vor allem in den neuen vorgestellten theoretischen Konzepten eine Rolle spielt. Dieses Verständnis wird beispielsweise auch vom Deutschen Olympischen Sportbund vertreten, der Integration als wechselseitigen Prozess und Dialog versteht und sich darüber hinaus nicht nur auf Personen mit Migrationshintergrund bezieht, sondern auch die Rolle der Aufnahmegesellschaft betont (DOSB 2014b: S. 8/9). Dies könnte dazu beitragen, dass diese Sichtweise vor allem in Sportvereinen an Bedeutung gewinnt.

Darüber hinaus wurden in der vorliegenden Arbeit in Bezug auf die Frage, inwiefern Sichtweisen auf Integration in Abhängigkeit von sozialstrukturellen Merkmalen und der Zusammensetzung der Mitglieder der Sportvereine variieren, folgende Hypothesen formuliert:

- H1: Das Alter der Befragten weist einen negativen Zusammenhang mit der Unterstützung pluralistischer Konzepte auf.

- H2: Personen mit Migrationshintergrund unterstützen pluralistische Konzepte stärker als andere Konzepte.

- H3: Umso höher der Anteil von Personen mit Migrationshintergrund in der Trainingsgruppe, desto stärker werden pluralistische Konzepte unterstützt.

- H4: Umso höher der Bildungsstand, desto stärker werden pluralistische Konzepte unterstützt.

Im Rahmen der Analysen konnten die Hypothesen H2 und H3 bestätigt werden.

Ein Zusammenhang der Unterstützung von Integrationskonzepten und Konstellation der Teilnehmenden, Alter und Migrationshintergrund zeigte sich in der Arbeit von Baur und Mutz (2009). Die Autoren konnten dabei feststellen, dass ältere Ansprechpersonen sowie Übungsleiterinnen und -leiter signifikant häufiger der Skala „Orientierung am Aufnahmeland“ zustimmen als jüngere Befragte der beiden Gruppen (Baur/Mutz 2009: 163). Im Rahmen der vorliegenden Arbeit konnte jedoch lediglich festgestellt werden, dass die Zustimmung zur Mehrfachintegration mit steigendem Alter sinkt, während die Befürwortung der Segregation steigt.

In Bezug auf den Indikator Migrationshintergrund zeigte sich bei Baur und Mutz, dass die Ansprechpersonen mit Migrationshintergrund eine höhere Zustimmung bei der Skala „Verleugnung der kulturellen Herkunft“ aufweisen, was auf einen erlebten Integrationsdruck zurückzuführen sein könnte. Übungsleiterinnen und -leiter mit Migrationshintergrund stimmen dem Konzept des Multikulturalismus signifikant häufiger zu als Übungsleiterinnen und -leiter 
ohne Migrationshintergrund (Baur/Mutz 2009: 164). In der vorliegenden Arbeit konnte ein Zusammenhang zwischen dem Merkmal Migrationshintergrund und der Befürwortung pluralistischer Integrationskonzepte aufgezeigt werden.

Der letzte Faktor, der im Rahmen der Analyse von Baur und Mutz Zusammenhänge aufwies, ist die Konstellation der Teilnehmenden. Hier zeigte sich, dass hohe Anteile von Zugewanderten in der Sportgruppe dazu führen, dass eine höhere Zustimmung für die Multikulturalismus-Skala vorliegt. Eine Befragung der Vereinsmitglieder enthielt die damalige Studie jedoch nicht. Hier ließ sich in der vorliegenden Arbeit ein Zusammenhang zwischen der Konstellation der Teilnehmenden und der Unterstützung pluralistischer Integrationskonzepte nachweisen.

In Bezug auf weitere sozialstrukturelle Merkmale zeigten sich für den Faktor Bildung, der in der Studie von Baur und Mutz (2009) nicht betrachtet wurde, negative Zusammenhänge mit dem Ausgang des Pluralismus und der Segregation.

Ein weiterer Aspekt, der im Rahmen dieser Arbeit untersucht, aber in der bisherigen Literatur vernachlässigt wurde, sind die Unterschiede zwischen Personen mit und ohne Migrationshintergrund bezüglich der Einschätzung von Dimensionen und Aspekten sowie Ergebnissen von Integration. In der quantitativen Analyse zeigten sich signifikante Unterschiede in der Bewertung einzelner Aspekte von Integration. Diese umfassen Chancen zur Teilnahme an der Gesellschaft, interethnische Kontakte und die rechtliche Gleichstellung, welche beide durch Personen mit Migrationshintergrund als wichtiger für Integration bewertet werden. Auffällig hierbei ist, dass es sich dabei nur um Aspekte handelt, die in Bezug zu anderen Personen stehen bzw. an deren Verwirklichung auch andere beteiligt sind. So bedürfen beispielsweise Chancen zur Teilnahme an der Gesellschaft einer gewissen Offenheit sowie entsprechender Möglichkeiten. Diese Aspekte können somit nicht durch die ,zu Integrierenden“ alleine erfüllt werden. Darüber hinaus zeigen sich auch signifikante Unterschiede zwischen Personen mit und ohne Migrationshintergrund in Bezug auf das Item „Es wäre gut, wenn alle Zuwanderer in Deutschland ihre Kulturen beibehielten“. Dieses wird von Personen mit Migrationshintergrund höher und damit als wünschenswerter bewertet. Dass dieser Wunsch nach Beibehaltung der Kultur bei Personen mit Migrationshintergrund eher besteht als bei Personen ohne Migrationshintergrund, ergibt sich vermutlich aus der eigenen Betroffenheit im Rahmen dieses Items. Während Personen mit Migrationshintergrund die Beibehaltung ihrer Kultur besonders wichtig ist, können Personen ohne Migrationshintergrund diese Aussage nicht vor dem Hintergrund einer eigenen Betroffenheit reflektieren und stellen damit nur eine Anforderung an Andere. Die Bewertung der Aspekte Chancen zur Teilnahme an 
der Gesellschaft, interethnische Kontakte und die rechtliche Gleichstellung als bedeutender für Integration durch Personen mit Migrationshintergrund, könnte darüber hinaus aus einer persönlichen Erfahrung mit Problematiken in diesen Bereichen einhergehen, die zu einer höheren Sensibilität in manchen Bereichen der Integration führen. Dass die persönlichen Erfahrungen bei Personen mit Migrationshintergrund eine bedeutende Rolle spielen, zeigt sich im Rahmen der vertiefenden Interviews. Dort zeigten sich zwar keine deutlichen Unterschiede in Ausführungen zu Aspekten und Dimensionen sowie Ausgängen von Integration, aber Personen mit Migrationshintergrund griffen immer wieder Erzählungen ihrer eigenen Erfahrungen mit Integration auf. Vor allem bei Personen mit eigener Migrationserfahrung scheint somit das Erleben der eigenen Integration eine bedeutende Rolle für das Sprechen über Integration zu spielen. Dieser Bezug auf die eigene Integrationserfahrung zeigte sich bereits bei Schramkowksi (2009) und führte, im Gegensatz zu den hier vorliegenden Interviews, zu einer negativen Konnotation des Integrationsbegriffs als Konzept der Anpassung. Somit ist anzunehmen, dass die eigene Erfahrung bei Personen mit Migrationshintergrund auch immer die Sichtweise auf dieses Konzept färbt.

Im Gegensatz dazu scheint Integration für Personen ohne Migrationshintergrund eher ein abstraktes Konstrukt darzustellen, das sie, ihrer Einschätzung nach, persönlich nicht betrifft und welches nicht vor dem Hintergrund einer eigenen Erfahrung reflektiert werden kann.

Das subjektive Integrationsverständnis weist in Bezug auf wünschenswerte Ausgänge von Integration somit nicht nur Zusammenhänge mit sozialstrukturellen Merkmalen wie Alter, Migrationshintergrund und Bildungsstand auf, sondern wird auch vor dem Hintergrund eigener Erfahrung reflektiert. Dies spielt vor allem bei Personen mit Migrationshintergrund eine Rolle. Aber auch bei Personen ohne Migrationshintergrund kann das Integrationsverständnis von persönlichen Erfahrungen beeinflusst werden. Bei diesen Erfahrungen spielen vor allem interethnische Kontakte eine Rolle, die zu einem Abbau von Vorurteilen führen.

Insgesamt zeigt sich jedoch auch, dass die Unterschiede zwischen Personen mit und ohne Migrationshintergrund eher gering sind. Dies könnte auf die selektive Stichprobe zurückzuführen sein, die sowohl bei Personen mit als auch ohne Migrationshintergrund überwiegend Personen mit einem hohen Bildungsstand aufweist.

Abschließend bleibt in Bezug auf die Ergebnisse dieser Arbeit festzuhalten, dass, im Rahmen der subjektiven Sichtweisen, Integration im Sinne eines interaktionistischen Ansatzes als beidseitiger Prozess verstanden wird, innerhalb dessen Anforderungen an unterschiedliche Akteurinnen und Akteure gestellt werden. Dabei kann zwischen als $\mathrm{zu}$ integrierend 
Wahrgenommenen und Handelnden unterschieden werden. Zwar liegt der Fokus bei als zu integrierend Wahrgenommenen auf Personen mit Migrationshintergrund, die weitere Fülle an angesprochenen Differenzkategorien weist aber wesentliche Bezüge zum Konzept der Diversität auf. Integration bezieht sich somit nicht ausschließlich auf Personen mit Migrationshintergrund. Darüber hinaus spielt vor allem die Dimension der Interaktion eine Rolle, während andere Integrationsdimensionen in den Hintergrund treten. Aspekte und Dimensionen von Integration stehen nicht einfach nebeneinander, sondern weisen Verflechtungen untereinander auf. Außerdem bleibt festzuhalten, dass der Assimilationsgedanke trotz der Wahrnehmung von Integration als beidseitiger Prozess nicht ganz an Bedeutung verliert und eher eine Mischung aus pluralistischen und assimilativen Ansichten in Bezug auf Ergebnisse von Integration vorliegt. Dies kann jedoch erneut auf eine interaktionistische Sichtweise hindeuten, innerhalb derer ein flexibler Prozess der gegenseitigen Anpassung und Akzeptanz besteht. 


\section{Mögliche Limitationen}

Im Rahmen möglicher Limitationen ist zu beachten, dass nur ein kleiner Teil der Mitglieder des Sportkreises Frankfurt erreicht werden konnten. Aufgrund der geringen Rücklaufquote sollten Ergebnisse daher nicht verallgemeinert werden und bei den Ergebnissen handelt es sich um eine erste Annäherung an den Bereich der subjektiven Sichtweisen auf Integration.

In der Stichprobe zeigte sich darüber hinaus eine schiefe Verteilung von Alter und Bildungsstand der Befragten. Die Stichprobe umfasste hauptsächlich Personen im Alter zwischen 21 und 30 Jahren sowie Personen mit einem hohen Bildungsstand. Da dies auch auf die in der Stichprobe vorhanden Personen mit Migrationshintergrund zutrifft, besteht auch innerhalb dieser Gruppe eine gewisse Selektivität.

Da auch innerhalb der Hypothesen angenommen wird, dass Personen mit einem höheren Bildungsstand Integration eher positiv gegenüberstehen bzw. pluralistische Konzepte unterstützen, könnte dies zu einer überwiegend positiven Bewertung in der Befragung geführt haben. Jedoch könnte diese Zusammensetzung auch spezifisch für Sportvereine sein. So zeigen beispielsweise Mutz und Burrmann (2015), dass die Mitgliedschaft in Sportvereinen einer gewissen Selektivität unterliegt und die Mitgliedschaftsquote mit höherer Bildung zunimmt (Mutz/Burrmann 2015: 86). Darüber hinaus könnte die Umfrage selbst, aufgrund des deutlichen Bezugs zu Integration, einer gewissen Selektivität unterliegen und überwiegend von Personen ausgefüllt worden sein, die eine positive oder offenere Einstellung zum Thema Integration aufweisen.

Die Analysen konnten aufgrund der kleinen Stichprobe nur mit Hilfe des paarweisen Fallausschlusses vorgenommen werden. Dadurch können den Berechnungen unterschiedliche Teilstichproben zugrunde liegen, auch wenn die Zahl der Fälle bei den meisten deskriptiv betrachteten Items keinen starken Schwankungen unterliegt.

Ebenfalls aufgrund der geringen Stichprobengröße werden Personen mit Migrationshintergrund als eine homogene Bevölkerungsgruppe dargestellt. Außerdem konnte nur anhand einer kleinen Substichprobe eine Unterscheidung zwischen Personen der ersten und zweiten Generation und somit mit und ohne eigene Migrationserfahrung vorgenommen werden. Hier wäre eine größere Stichprobe sowie eine weitere Differenzierung wünschenswert, um Unterschiede zwischen den Generationen sowie zwischen einzelnen Gruppen darstellen zu können. 


\section{Literaturverzeichnis}

Aigner, Petra (2017): Migrationssoziologie. Eine Einführung, Wiesbaden: Springer VS. Alba, Richard/ Nee, Victor (2003): Remaking the American Mainstream. Assimilation and Contemporary Immigration, Cambridge: Harvard University Press.

Allport, Gordon W. (1954): The Nature of Prejudice, Reading: Addison-Wesley.

Anhut, Reimund/ Heitmeyer, Wilhelm (2009): Desintegration, Anerkennungsbilanzen und die Rolle sozialer Vergleichsprozesse für unterschiedliche Verarbeitungsmuster, in: Preyer (2009): Neuer Mensch und kollektive Identität in der Kommunikationsgesellschaft, Wiesbaden: VS Verlag für Sozialwissenschaften, S. 212-236.

Baur, Nina/ Kelle, Udo/ Kuckartz, Udo (2017): Mixed Methods - Stand der Debatte und aktuelle Problemlagen, in: Kölner Zeitschrift für Soziologie, Sonderhefte, 57, S. $1-37$.

Baur, Jürgen/ Mutz, Michael (2009): Subjektive Integrationskonzepte der Ansprechpartner und Übungsleiter, in: Baur et al.: Evaluation des Programms „Integration durch Sport“, Band 1, Potsdam, S. 155-181.

Becker, Simone/ Häring, Armando (2012): Soziale Integration durch Sport? Eine empirische Analyse zum Zusammenhang von Sport und sozialer Integration, in: Sportwissenschaft, 42, S. 261-270.

Berry, John W. (1997). Immigration, Acculturation, and Adaptation, in: Applied Psychology: An International Review, 46(1), S. 5-34.

Berry, John W. (1990): Psychology of Acculturation. Understanding Individuals Moving between Cultures, in: Brislin, Richard W. (Hrsg.): Applied Cross-Cultural Psychology, Newbury Park/London/New Delhi: Sage, S. 232- 253.

Berry, John W./ Kim, Uichol/ Power, Simon/ Young, Marta/ Bujaki, Merridee (1989). Acculturation Attitudes in Plural Societies, in: Applied Psychology: An International Review, 38, S. 185 - 206.

Blohm, Michael/ Wasmer, Martina (2018): Einstellungen und Kontakte zu Ausländern, in: Statistisches Bundesamt/ Wissenschaftszentrum Berlin für Sozialforschung: Datenreport 2018, S. 403-411. 
Braun, Sebastian/ Nobis, Tina (2011): Migration, Integration und Sport - Perspektiven auf zivilgesellschaftliche Kontexte vor Ort - Zur Einführung, in: Braun, Sebastian/ Nobis, Tina (2011): Migration, Integration und Sport. Zivilgesellschaft vor Ort, VS Verlag: Wiesbaden, S. 9-28.

Braun, Sebastian/Finke, Sebastian (2010): Integrationsmotor Sportverein. Ergebnisse zum Modellprojekt „spin - sport interkulturell“, VS Verlag: Wiesbaden.

Braun, Sebastian (2006): Soziale und politische Integration durch Vereine? Theoretische Ansätze und empirische Ergebnisse, in: Rehberg, Karl-Siegberg (Hrsg.): Soziale Ungleichheit, kulturelle Unterschiede: Verhandlungen des 32. Kongresses der Deutschen Gesellschaft für Soziologie in München, Teilband. 1 und 2, Frankfurt am Main: Campus Verlag, S. 4498-4508.

Breuer, Christoph/ Feiler, Svenja (2017a): Integrationsleistungen der Sportvereine in Deutschland, in: Breuer, Christoph (Hrsg.) (2017): Sportentwicklungsbericht 2015/2016, Analyse zur Situation der Sportvereine in Deutschland, Sportverlag Strauß: Hellenthal, S. 101-194.

Breuer, Christoph/ Feiler, Svenja (2017b): Methode, in: Breuer, Christoph (Hrsg.) (2017): Sportentwicklungsbericht 2015/2016, Analyse zur Situation der Sportvereine in Deutschland, Sportverlag Strauß: Hellenthal, S. 780-803.

Breuer, Christoph/ Feiler, Svenja (2015): Integration von Migranten im Sportverein, in: Breuer, Christoph (Hrsg.) (2015): Sportentwicklungsbericht 2013/2014, Analyse zur Situation der Sportvereine in Deutschland, Sportverlag Strauß: Köln, S. 231257.

Bundesamt für Migration und Flüchtlinge (Bamf) (2015): Migrationsbericht des Bundesamts für Migration und Flüchtlinge im Auftrag der Bundesregierung. Berlin.

Bundesregierung (2007): Der Nationale Integrationsplan. Neue Wege - Neue Chancen, unter:

https://www.bundesregierung.de/Content/DE/Archiv16/Artikel/2007/07/Anlage /2007-08-30-nationaler-integrationsplan.pdf?_blob=publicationFile\&v=1 (Aufruf 02.07.2018). 
Bundesregierung (2008): Nationaler Integrationsplan. Erster Fortschrittsbericht, unter: http://www.bundesregierung.de/Content/DE/Publikation/IB/Anlagen/nationaler -integrationsplan-fortschrittsbericht.pdf?_blob=publicationFile_ (Aufruf 02.07.2018).

Bundesregierung (2011): Nationaler Aktionsplan Integration. Zusammenhalt stärken Teilhabe verwirklichen, unter: https://www.bundesregierung.de/Content/DE/_Anlagen/IB/2012-01-31-napgesamt-barrierefrei.pdf?__blob=publicationFile (Aufruf 02.07.2018).

Bundesregierung (o.J.): Nationaler Integrationsplan, unter: https://www.nationaleraktionsplan-integration.de/ (Aufruf: 09.10.2020).

Burrmann, Ulrike (2014): Integration aus (sport-)soziologischer Perspektive, in: GießStüber, Petra/ Burrmann, Ulrike/ Radtke, Sabine/ Rulofs, Bettina/ Tiemann, Heike: Expertise, Diversität, Inklusion, Integration und Interkulturalität. Leitbegriffe der Politik, sportwissenschaftliche Diskurse und Empfehlungen für den DOSB und die dsj, Frankfurt: DOSB, S. 21-27.

Burrmann, Ulrike/ Mutz, Michael/ Zender, Ursula (2011): Integration von Jugendlichen mit Migrationshintergrund: Ein empirisch fundierter Vergleich zwischen Sportvereinen und Schulsport-AGs, in: Sportunterricht, 60 (2011) 8, S. 259-262.

Ceobanu, Alin M./ Escandell, Xavier (2010): Comparative Analyses of Public Attitudes Toward Immigrants and Immigration using Multinational Survey Data: A Review of Theories and Research, in: Annual Review of Sociology, S. 309-328.

Civitillo, Sauro/ Schachner, Maja/ Juang, Linda/ van de Vijver, Fons J. R./ Handrick, Anna/ Noack, Peter (2017): Towards a better understanding of cultural diversity approaches at school: A multi-informant and mixed-methods study. Learning, Culture and Social Interaction 12: S. 1-14.

Civitillo, Sauro/ Juang, Linda/ Badra, Marcel/ Schachner, Maja (2019): The interplay between culturally responsive teaching, cultural diversity beliefs, and selfreflection: A multiple case study. Teaching and Teacher Education 77: S. 341351.

Creswell, John W./ Plano Clark, Vicki L./ Gutmann, Michelle L./ Hanson, William E. (2003): Advanced mixed methods research designs, in Tashakkori, Abbas M./ 
Teddlie, Charles B. (Hrsg.): Handbook of mixed methods in social and behavioral research, Thousand Oaks: Sage S. 209-240.

Deutscher Olympischer Sportbund (DOSB) (2020): Bestandserhebung 2019 - Fassung vom $13 . \quad$ Januar unter: https://cdn.dosb.de/user_upload/www.dosb.de/medien/BE/BE-Heft_2019.pdf (Aufruf 27.10.2020).

Deutscher Olympischer Sportbund (DOSB) (2014a): Integration durch Sport Programmkonzeption, unter: https://cdn.dosb.de/user_upload/www.integrationdurchsport.de/Service/InfoMaterial/Programmkonzeption_Integration_durch_Sport.p df (Aufruf 13.06.2018).

Deutscher Olympischer Sportbund (DOSB) (2014b): Integration und Sport - Ein Zukunftsfaktor von Sportvereinen und Gesellschaft. Grundlagenpapier, unter: https://cdn.dosb.de/user_upload/www.integration-durch-sport.de/Service/InfoMaterial/Grundlagenpapier.pdf (Aufruf 20.11.2018).

Düvell, Frank (2006): Europäische und internationale Migration. Einführung in historische, soziologische und politische Analysen, Hamburg: Lit Verlag.

Eisenstadt, Shmuel. N. (1951): The Place of Elites and Primary Groups in the Absorption of New Immigrants in Israel, in: American Journal of Sociology, 57, 2/1951, S. 222-223.

Eisenstadt, Shmuel N. (1954): The Absorption of Immigrants. A comparative study based mainly on the jewish community in Palestine and the State of Israel, London: Routledge \& Paul.

Eisenstadt, Shmuel N. (1952a): The process of absorption of new immigrants in Israel, in: Human Relations, Vol. 3, Issue 3, S. 223-246.

Eisenstadt, Shmuel N. (1952b): Institutionalization of Immigrant Behaviour, in: Human Relations, Vol. 5, S. 373-395.

Eisenstadt, Shmuel. N. (1951): The Place of Elites and Primary Groups in the Absorption of New Immigrants in Israel, in: American Journal of Sociology, 57, 2/1951, S. 222-223. 
Esser, Hartmut (2006): Sprache und Integration. Die sozialen Bedingungen und Folgen des Spracherwerbs von Migranten. Frankfurt a. M.: Campus Verlag.

Esser, Hartmut (2001): Integration und ethnische Schichtung, Arbeitspapiere Mannheimer Zentrum für Europäische Sozialforschung, 40.

Esser, Hartmut (1999). Inklusion, Integration und ethnische Schichtung, in: Journal für Konflikt- und Gewaltforschung, 1, S. 5-34.

Eurostat (2016a): Migration and migrant population statistics: Tables and figures, unter: http://ec.europa.eu/eurostat/statisticsexplained/index.php/Migration_and_migra nt_population_statistics\#Further_Eurostat_information(Aufruf 13.06.2018).

Faist, Thomas (2000): Jenseits von Nation und Postnation. Eine neue Perspektive für die Integrationsforschung, in: Faist, Thomas (Hrsg.): Politik, Wirtschaft und Kultur in und zwischen Deutschland und der Türkei, Bielefeld: Transcript Verlag.

Fussan, Nancy (2006): Einbindung Jugendlicher in Peer-Netzwerke. Welche Integrationsvorteile erbringt die Mitgliedschaft in Sportvereinen?, in: Zeitschrift für Soziologie der Erziehung und Sozialisation, 26 (2006) 4, S. 383-402.

Gans, Herbert J. (1997): Toward a Reconciliation of "Assimilation" and "Pluralism": The Interplay of Acculturationand Ethnic Retention, in: The International Migration Review, Vol. 31, No. 4, Special Issue: Immigrant Adaptation and Native-Born Responses in the Making of Americans (Winter, 1997), S.875-892.

Gieß-Stüber, Petra/ Burrmann, Ulrike/ Radtke, Sabine/ Rulofs, Bettina/ Tiemann, Heike: Diversität, Inklusion, Integration und Interkulturalität, Bewährte Strategien und Entwicklungsperspektiven für den organisierten Sport, in: Gieß-Stüber, Petra/ Burrmann, Ulrike/Radtke/Rulofs, Bettina/Tiemann, Heike: Expertise, Diversität, Inklusion, Integration und Interkulturalität. Leitbegriffe der Politik, sportwissenschaftliche Diskurse und Empfehlungen für den DOSB und die dsj, Frankfurt: DOSB, S. 35-42.

Glaser, Barney G./ Strauss, Anselm L. (2010): Grounded Theory: Strategien qualitativer Forschung, 3. Auflage, Bern: Huber.

Glazer, Nathan/ Moynihan, Daniel P. (1970): Beyond the Melting Pot: The Negroes, Puerto Ricans, Jews, Italians, and Irish of New York City, 2. Auflage, Cambridge: MIT Press. 
Gordon, Milton M. (1964): Assimilation in American Life. The Role of Race, Religion and National Origins. New York: Oxford University Press.

Greene, Jennifer/ Caracelli, Valerie/ Graham, Wendy (1989): Toward a conceptual framework for mixed-methods evaluation designs, in: Educational Evaluation and Policy Analysis, Vol. 11, Nr. 3, S. 255-274.

Hachfeld, Axinja/ Hahn, Adam/ Schroeder, Sascha/ Anders, Yvonne/ Stanat, Petra/ Kunter, Mareike (2011): Assessing Teachers' Multicultural and Egalitarian Beliefs: The Teacher Cultural Beliefs Scale. Teaching and Teacher Education 27(6): S. 986-996.

Hachfeld, Axinja/ Schroeder, Sasche/ Anders, Yvonne/ Hahn, Adam/ Kunter, Mareike (2012): Herkunft oder Über-zeugung? Welche Rolle spielen der Migrationshintergrund und multikulturelle Überzeugungen für das Unterrichten von Kindern mit Migrationshintergrund? Zeitschrift für Pädagogische Psychologie 26(2): S. 101-120.

Hachfeld, Axinja, Adam Hahn, Sascha Schroeder, Yvonne Anders und Mareike Kunter (2015): Should teachers be colorblind? How multicultural and egalitarian beliefs differentially relate to aspects of teachers' professional competence for teaching in diverse classrooms. Teaching and Teacher Education 48: S. 44-55.

Han, Petrus (2010): Soziologie der Migration. Erklärungsmodelle, Fakten, politische Konsequenzen, Perspektiven. Stuttgart: UTB.

Hanhart, Susanne (2020): Migration in den Vorstellungen von Grundschulkindern. Wahrnehmung und Bewertung gesellschaftlicher und individueller Veränderungsprozesse, Wiesbaden: Springer VS.

Hans, Silke (2016): Theorien der Integration von Migranten - Stand und Entwicklung, in: Brinkmann, Heinz U./ Sauer, Martina (Hrsg.): Einwanderungsgesellschaft Deutschland. Wiesbaden: Springer Fachmedien.

Haug, Sonja (2006): Interethnische Freundschaften, interethnische Partnerschaften und soziale Integration, in: Diskurs Kindheits- und Jugendforschung, Heft 1-2006, S. 75-91.

Heckmann, Friedrich (2015): Integration von Migranten. Einwanderung und neue Nationenbildung. Wiesbaden: Springer VS. 
Heckmann, Friedrich (1985): Sport und die gesellschaftliche Integration von Minderheiten, in: Friedrich Ebert Stiftung (Hrsg.): Sport und ausländische Mitbürger, Bonn, S. 21-33.

Heckmann, Friedrich (1997): Integration und Integrationspolitik in Deutschland, Beitrag zum Internationalen Forum „Migration und Mittelmeer“, Friedrich-EbertStiftung, europäisches Forum für Migrationsstudien.

Heckmann, Friedrich/ Wunderlich, Tanja/ Worbs, Susanne/ Lederer, Harald W. (2000): Integrationspolitische Aspekte einer gesteuerten Zuwanderung, Gutachten für die interministerielle Arbeitsgruppe der Bayerischen Staatsregierung zu Fragen der Zuwanderungssteuerung und Zuwanderungsbegrenzung, europäisches Forum für Migrationsstudien.

Heitmeyer, Wilhelm/ Anhut, Reimund (Hrsg.) (2000): Bedrohte Stadtgesellschaft. Soziale Desintegrationsprozesse und ethnisch-kulturelle Konfliktkonstellationen, Weinheim: Juventa.

Hessisches Ministerium der Justiz, für Integration und Europa (2012): Integrationspolitik der Hessischen Landesregierung, unter: http://www.integrationskompass.de/global/show_document.asp?id=aaaaaaaaaaa cyhf (Aufruf 02.07.2018).

Hessisches Ministerium der Justiz, für Integration und Europa (2013): Landesprogramm Modellregionen Integration, unter: http://www.integrationskompass.de/global/show_document.asp?id=aaaaaaaaaaa dfhj (Aufruf 03.07.2018).

Hoffmann-Nowotny, Hans-Joachim (1973): Soziologie des Fremdarbeiterproblems, Eine theoretische und empirische Analyse am Beispiel der Schweiz, Stuttgart: Ferdinand Enke Verlag.

Hoffmann-Nowotny, Hans-Joachim (1990): Integration, Assimilation und" plurale Gesellschaft". Konzeptuelle, theoretische und praktische Überlegungen, in: Höhn, Charlotte/ Rein, Detlev B. (Hrsg.): Ausländer in der Bundesrepublik Deutschland, Boppard/Rhein: Boldt, S. 15-31.

Kelle, Udo (2017): Die Integration qualitativer und quantitativer Forschung Theoretische Grundlagen von Mixed Methods, in: Kölner Zeitschrift für 
Soziologie, Sonderhefte, 57, S. 39-61.

Kuckartz, Udo (2014): Mixed Methods. Methodologie, Forschungsdesigns und Analyseverfahren, Wiesbaden: Springer VS.

Lienert, Gustav/ Raatz, Ullrich (1994): Testaufbau und Testanalyse. Weinheim: Beltz.

Lockwood, David (1964): Social Integration and System Integration, in: George K. Zollschan, George K./ Hirsch, Walter (Hrsg.): Explorations in Social Change, Houghton Mifflin Company: Boston.

Lutter, Andreas (2011): Integration im Bürgerbewusstsein von Schülern, Wiesbaden: Springer VS.

Maehler, Déborah/ Teltemann, Janna/ Rauch, Dominique/ Hachfeld, Axinja (2016): Die Operationalisierung des Migrationshintergrundes, in: Maehler, Débora/ Brinkmann, Heinz Ulrich (Hrsg.): Methoden der Migrationsforschung. Ein interdisziplinärer Forschungsleitfaden, Wiesbaden: Springer VS.

Mayring, Philipp (2008): Qualitative Inhaltsanalyse. Grundlagen und Techniken, 10. Auflage, Weinheim/Basel: Beltz Verlag.

Mayring, Philipp (2000). Qualitative Content Analysis, in: Forum Qualitative Social Research, 1(2), Art. 20.

Mutz (2018): Sport als Sprungbrett in die Gesellschaft. Sportengagements von Jugendlichen mit Migrationshintergrund und ihre Wirkung, Weinheim/Basel Beltz/Juventa.

Mutz, Michael/ Burrmann, Ulrike (2015): Zur Beteiligung junger Migrantinnen und Migranten am Vereinssport, in: Burrmann et al. (2015): Jugend, Migration und Sport, Wiesbaden: Springer Fachmedien.

Mutz, Michael (2013): Expertise - DOSB, Die Partizipation von Migrantinnen und Migranten am vereinsorganisierten Sport, Frankfurt: DOSB.

Nobis, Tina/ Strahle, Yvonne (2009): Integrationskonzepte: Einleitende Anmerkungen, in: Baur et al (2009).: Evaluation des Programms „Integration durch Sport“ Band 1, S. 117-131. 
Nobis, Tina/Fussan, Nancy (2007): Soziale Integration von Jugendlichen mit Migrationshintergrund: Vorbemerkungen zur Bedeutung der Sportvereine, in: Nobis, Tina/ Baur, Jürgen (2007): Soziale Integration vereinsorganisierter Jugendlicher, Bundesinstitut für Sportwissenschaft, Wissenschaftliche Berichte und Materialien, Band 09/2007, Sportverlag Strauß: Köln, S. 261-276.

Oswald, Ingrid (2007): Migrationssoziologie, Konstanz: UVK Verlagsgesellschaft mbH.

Portes, Alejandro/ Zhou, Min (1993): The New Second Generation: Segmented Assimilation and Its Variants, in: The Annals of the American Academy of Political and Social Science, Vol. 530, Interminority Affairs in the U. S.: Pluralism at the Crossroads (Nov. 1993), S. 74-96.

Park, Robert E./ Burgess, Ernest W. (1969, 1921): Introduction to the Science of Sociology, Chicago: University of Chicago Press.

Pettigrew, Thomas F. (1986): The intergroup contact hypothesis reconsidered, in: Hewstone, Miles/ Brown, Rupert (Hrsg.) (1986): Contact and conflict in intergroup encounters, Oxford: Blackwell, S. 169-195.

Rasch, Björn/ Friese, Malte/ Hofmann, Wilhelm/ Naumann, Ewald (2014a): Quantitative Methoden. 1. Einführung in die Statistik für Psychologen und Sozialwissenschaftler, 4. Auflage, Berlin/Heidelberg: Springer Verlag.

Rasch, Björn/ Friese, Malte/ Hofmann, Wilhelm/ Naumann, Ewald (2014b): Quantitative Methoden. 2. Einführung in die Statistik für Psychologen und Sozialwissenschaftler, 4. Auflage, Berlin/Heidelberg: Springer Verlag.

Reuter, Julia/ Mecheril, Paul (2015): Schlüsselwerke der Migrationsforschung. Pionierstudien und Referenztheorien, Wiesbaden: Springer VS.

Rübner, Anne/ Strahle, Yvonne/ Baur, Jürgen (2009): Befragung der Landeskoordinatoren: Erhebungsdesign, in: Baur et al. (2009): Evaluation des Programms „Integration durch Sport“, Band 1, Potsdam, S. 57-78.

Rulofs, Bettina (2014): „Diversität“ und „Diversitäts-Management“ - Auslegungen der Konzepte für die Entwicklung von Sportorganisationen, in: Gieß-Stüber, Petra/ Burrmann, Ulrike/ Radtke, Sabine/ Rulofs, Bettina/ Tiemann, Heike: Expertise, Diversität, Inklusion, Integration und Interkulturalität. Leitbegriffe der Politik, sportwissenschaftliche Diskurse und Empfehlungen für den DOSB und die dsj, 
Frankfurt: DOSB, S. 7-13.

Sen, Faruk/ Sauer, Martina/ Halm, Dirk (2001): Integratives Verhalten und (Selbst-) Ethnisierung von türkischen Zuwanderern. Gutachten des ZfT für die Unabhängige Kommission „Zuwanderung“, in: Goldberg, Andreas/ Halm, Dirk/ Sauer, Martina (Hrsg.): Migrationsbericht des Zentrums für Türkeistudien 2002, Münster u.a.: LIT Verlag, S. 11-126.

Schacht, Diana/ Kristen, Cornelia/ Tucci, Ingrid (2014): Interethnische Freundschaften in Deutschland, in: Kölner Zeitschrift für Soziologie, 66, S. 445-458.

Schramkowski, Barbara (2009): „Für mich aber hat dieses Integrationswort mit der Zeit seinen Wert verloren." Perspektiven junger Erwachsener mit Migrationshintergrund, in: Geisen, Thomas/ Riegel, Christine Hrsg.): Jugend, Partizipation und Migration. Orientierungen im Kontext von Integration und Ausgrenzung, 2. durchgesehene Auflage, Wiesbaden: VS Verlag. S. 149-167.

Schramkowski, Barbara (2007): Integration unter Vorbehalt. Perspektiven junger Erwachsener mit Migrationshintergrund, Frankfurt a.M./London: IKO-Verlag.

Schulte, Axel (2000): Multikulturelle Gesellschaft: Chance, Ideologie oder Bedrohung, in: Zwischen Diskriminierung und Demokratisierung. Aufsätze zu Politiken und Migration, Integration und Multikulturalität in Westeruopa, Frankfurt a.M.: IKO Verlag, S. 199-227.

Soeffer, Hans-Georg/ Zifonun, Darius (2005): Integration - eine wissenssoziologische Skizze, in: Heitmeyer, Wilhelm/ Imbusch, Peter: Integrationspotenziale einer modernen Gesellschaft, Wiesbaden: Verlag für Sozialwissenschaften.

Statistisches Bundesamt (2019): Statistisches Jahrbuch Deutschland 2018, Wiesbaden. Statistisches Bundesamt (2018): Statistisches Jahrbuch Deutschland 2017, Wiesbaden. Statistisches Bundesamt (2017): Statistisches Jahrbuch Deutschland 2016, Wiesbaden. Statistisches Bundesamt (2016): Statistisches Jahrbuch Deutschland 2015, Wiesbaden. Statistisches Bundesamt (2015): Statistisches Jahrbuch Deutschland 2014, Wiesbaden. Strahle, Yvonne/ Baur, Jürgen (2009): Subjektive Integrationskonzepte der Landeskoordinatoren, in: Baur et al. (2009): Evaluation des Programms 
„Integration durch Sport“, Band 1, Potsdam, S. 131-154.

Petschel, Anja/ Will, Anne-Kathrin (2020): Migrationshintergrund - Ein Begriff, viele Definitionen, in: WISTA, 5, S. 78-90, unter: https://www.destatis.de/DE/Methoden/WISTA-Wirtschaft-undStatistik/2020/05/wista052020.pdf;jsessionid=79D3666350D1C6628DF3496CFA978B7A.internet871 1?__blob=publicationFile (Aufruf 19.11.2020).

Van Dick, Rolf/ Wagner, Ulrich/ Adams, Claudia/ Petzel, Thomas (2006): Einstellungen zur Akkulturation, in: Glöckner-Rist, Angelika (Hrsg.): ZIS-Version 10.00, Mannheim: Zentrum für Umfragen, Methoden und Analysen.

Van Dick, Rolf/ Wagner, Ulrich/ Adams, Claudia/ Petzel, Thomas (1997): Erste Evaluation eines Fragebogens an sechs deutschen Stichproben, in: Gruppendynamik (1997), Heft 1, Jg. 28, S. 83-92.

Völkl, Kerstin/ Korb, Christoph (2018): Variablen und Skalenniveaus, in: Deskriptive Statistik. Elemente der Politik, Wiesbaden: Springer VS.

Wirtz, Markus Antonius (2004): Über das Problem fehlender Werte: Wie der Einfluss fehlender Informationen auf Analyseergebnisse entdeckt und reduziert werden kann, in: Die Rehabilitation, 43 (2), S. 109-115.

Zick, Andreas und Madlen Preuß (2016): Einstellungen zur Integration in der Bevölkerung. Kurzbericht zum Projekt ZuGleich - Zugehörigkeit und Gleichwertigkeit, unter: http://ekvv.unibielefeld.de/blog/ikgblog/resource/ZuGleich-Kurzbericht_2016.pdf 


\section{Anhang}

Tabelle 29: Sportvereinen in Hessen in den Jahren 2014 bis 2018

\begin{tabular}{|l|l|l|l|l|}
\hline $\mathbf{2 0 1 4}$ & $\mathbf{2 0 1 5}$ & $\mathbf{2 0 1 6}$ & $\mathbf{2 0 1 7}$ & $\mathbf{2 0 1 8}$ \\
\hline 7.754 & 7.746 & 7.723 & 7.674 & 7.629 \\
\hline
\end{tabular}

Quelle: Eigene Darstellung nach Statistischen Jahrbüchern 2019-2015.

Tabelle 30: Mitglieder in Sportvereinen in Hessen in den Jahren 2014 bis 2018

\begin{tabular}{|l|l|l|l|l|}
\hline $\mathbf{2 0 1 4}$ & $\mathbf{2 0 1 5}$ & $\mathbf{2 0 1 6}$ & $\mathbf{2 0 1 7}$ & $\mathbf{2 0 1 8}$ \\
\hline 2.039 .871 & 2.058 .323 & 2.071 .228 & 2.081 .800 & 2.093 .052 \\
\hline
\end{tabular}

Quelle: Eigene Darstellung nach Statistischen Jahrbüchern 2019-2015.

Tabelle 31: Darstellung der Items und Skalen aus der Studie Baur/Mutz 2015

\section{Skala 1: Orientierung am Aufnahmeland}

(Cronbach's alpha $=0.60, \mathrm{~N}=903$ )

- Ausländer, die in Deutschland leben, sollten ihr Verhalten der deutschen Lebensweise anpassen (van Dick et al., 2006).

- Ausländer, die in Deutschland leben, sollten auch die deutsche Sprache sprechen (Heitmeyer/ Anhut 2000).

- Ausländer, die in Deutschland leben, sollten sich auch für die deutsche Kultur interessieren (Heitmeyer/ Anhut 2000).

- Nach Deutschland immigrierte Menschen, sollen ihre Kinder so erziehen, dass sie vorwiegend deutschsprachig aufwachsen (van Dick et al., 2006).

\section{Skala 2: Verleugnung der kulturellen Herkunft}

(Cronbach's alpha $=0.57, \mathrm{~N}=903$ )

- Es wäre gut, wenn Ausländer, die in Deutschland leben, ihre Kultur so schnell wie möglich zurückstellen würden (van Dick et al., 2006).

- Gibt es verschiedene ethnische Gruppen in Deutschland, wird es schwierig, Probleme zu lösen (van Dick et al., 2006).

- Ausländer sollten ihre fremdkulturellen Gewohnheiten in der Öffentlichkeit nicht zeigen (van Dick et al., 2006).

\section{Skala 3: Multikulturalismus}

(Cronbach's alpha $=0.63, \mathrm{~N}=903$ )

- Es wäre gut, wenn alle ethnischen Gruppen in Deutschland ihre Kultur beibehielten (van Dick et al., 2006).

- Das Zusammenleben verschiedener ethnischer Gruppen in Deutschland wäre leichter, wenn die Mitglieder der verschiedenen Gruppen Gelegenheit hätten, ihren eigenen Lebensstil beizubehalten (van Dick et al., 2006). 
- Eine Gesellschaft mit einer Vielzahl ethnischer Gruppen ist eher befähig, neue Probleme in Angriff zu nehmen (van Dick et al., 2006).

Sportbezogenes Integrationskonzept

- Es ist die Aufgabe der Sportvereine, Angebote zu schaffen, die den Sportinteressen der Zuwanderer entsprechen.

- Wenn Zuwanderer und Einheimische gemeinsam Sport treiben, findet Integration von ganz alleine statt.

- Sportgruppen, sie zu 100\% aus Zuwanderern bestehen, sind keine Integrationsgruppen.

Quelle: Baur/ Mutz 2015, S. 156/158.

Tabelle 32: Mitgliedschaften und Organisationsgrad unterschiedlicher Altersgruppen in Sportvereinen 2019

\begin{tabular}{|l|l|l|l|}
\hline Altersgruppe & $\begin{array}{l}\text { Anzahl } \\
\text { Mitgliedschaften 2019 }\end{array}$ & $\begin{array}{l}\text { Anteil Mitgliedschaften } \\
\mathbf{2 0 1 9} \text { in Prozent }\end{array}$ & $\begin{array}{l}\text { Organisationsgrad } \\
\text { bezogen auf Anteil in der } \\
\text { Bevölkerung in Prozent }\end{array}$ \\
\hline Bis 6 Jahre & 1.352 .441 & 5,6 & 25,0 \\
\hline 7 bis 14 Jahre & 4.163 .897 & 17,4 & 70,5 \\
\hline 15-18 Jahre & 1.750 .930 & 7,3 & 55,4 \\
\hline 19 bis 26 Jahre & 2.412 .798 & 10,1 & 32,2 \\
\hline 27 bis 40 Jahre & 3.567 .868 & 14,9 & 23,8 \\
\hline 41 bis 60 Jahre & 6.174 .613 & 25,7 & 25,6 \\
\hline Über 60 Jahre & 4.570 .833 & 19,0 & 21,2 \\
\hline
\end{tabular}

Quelle: DOSB 2020 
Tabelle 33: Test auf Normalverteilung der verwendeten Items zu Aspekten und Ausgängen von Integration

Item

p p Shapiro-Wilk

Kolmogorov

-Smirnov ${ }^{\mathbf{a}}$

\begin{tabular}{|l|l|l}
\hline Gut Deutsch sprechen & 0,000 & 0,000 \\
\hline Anerkennung wichtiger Werte & 0,000 & 0,000 \\
\hline Chancen zur Teilnahme an der Gesellschaft & 0,000 & 0,000 \\
\hline Der Wille, an der Gesellschaft teilzunehmen & 0,000 & 0,000 \\
\hline Kontakte zwischen Menschen verschiedener Kulturen & 0,000 & 0,000 \\
\hline $\begin{array}{l}\text { Mitgliedschaft/ } \\
\text { Engagement in Organisationen }\end{array}$ & 0,000 & 0,000 \\
\hline Anerkennung durch Andere & 0,000 & 0,000 \\
\hline $\begin{array}{l}\text { Vorhandensein eines Zugehörigkeitsgefühls } \\
\text { Gleiche Rechte für Alle }\end{array}$ & 0,000 & 0,000 \\
\hline Bildungsstand & 0,000 & 0,000 \\
\hline Berufliche Stellung & 0,000 & 0,000 \\
\hline $\begin{array}{l}\text { Zugang zum Wohnungsmarkt } \\
\text { Zufriedenheit mit beruflicher und sozialer Situation }\end{array}$ & 0,000 & 0,000 \\
\hline $\begin{array}{l}\text { Wenn Zuwanderer ihre Kultur beibehalten möchten, } \\
\text { sollten sie unter sich bleiben }\end{array}$ & 0,000 & 0,000 \\
\hline $\begin{array}{l}\text { Es wäre gut, wenn alle Zuwanderer in Deutschland } \\
\text { ihre Kulturen beibehielten }\end{array}$ & 0,000 & 0,000 \\
\hline $\begin{array}{l}\text { Menschen, die nach Deutschland kommen, sollten ihr } \\
\text { Verhalten der deutschen Kultur anpassen }\end{array}$ & 0,000 & 0,000 \\
\hline $\begin{array}{l}\text { Zuwanderer sollten sowohl in ihre } \\
\text { Herkunftsgesellschaft als auch in die deutsche } \\
\text { Gesellschaft eingebunden sein }\end{array}$ & 0,000 & 0,000 \\
\hline $\begin{array}{l}\text { Quelle: Eigene Darstellung; Anmerkungen: a Lilliefors Signifikanz Korrektur; } \\
\text { Unterscheidung der Gruppen mit und ohne Migrationshintergrund sowie }\end{array}$ & Vorstände und Mitglieder. \\
\hline
\end{tabular}

Tabelle 34: Levene-Test auf Varianzhomogenität der Items zu Aspekten und Ausgängen von Integration für Personen mit und ohne Migrationshintergrund

\begin{tabular}{|l|l|}
\hline Item & Sig, \\
\hline Gut Deutsch sprechen & 0,849 \\
\hline Anerkennung wichtiger Werte & 0,205 \\
\hline Chancen zur Teilnahme an der Gesellschaft & 0,761 \\
\hline Der Wille, an der Gesellschaft teilzunehmen & 0,947 \\
\hline Kontakte zwischen Menschen verschiedener Kulturen & $\mathbf{0 , 0 3 2}$ \\
\hline $\begin{array}{l}\text { Mitgliedschaft/ } \\
\text { Engagement in Organisationen }\end{array}$ & 0,545 \\
\hline Anerkennung durch Andere & 0,269 \\
\hline Vorhandensein eines Zugehörigkeitsgefühls & 0,949 \\
\hline
\end{tabular}




\begin{tabular}{|l|l|}
\hline Gleiche Rechte für Alle & $\mathbf{0 , 0 0 1}$ \\
\hline Bildungsstand & 0,383 \\
\hline Berufliche Stellung & 0,640 \\
\hline Zugang zum Wohnungsmarkt & 0,620 \\
\hline Zufriedenheit mit beruflicher und sozialer Situation & 0,113 \\
\hline $\begin{array}{l}\text { Wenn Zuwanderer ihre Kultur beibehalten möchten, } \\
\text { sollten sie unter sich bleiben }\end{array}$ & 0,317 \\
\hline $\begin{array}{l}\text { Es wäre gut, wenn alle Zuwanderer in Deutschland } \\
\text { ihre Kulturen beibehielten }\end{array}$ & 0,073 \\
\hline $\begin{array}{l}\text { Menschen, die nach Deutschland kommen, sollten ihr } \\
\text { Verhalten der deutschen Kultur anpassen }\end{array}$ & 0,941 \\
\hline $\begin{array}{l}\text { Zuwanderer sollten sowohl in ihre } \\
\text { Herkunftsgesellschaft als auch in die deutsche } \\
\text { Gesellschaft eingebunden sein }\end{array}$ & 0,815 \\
\hline \begin{tabular}{l} 
Quelle: Eigene Darstellung. \\
\hline
\end{tabular} & \\
\hline
\end{tabular}

Tabelle 35: Levene-Test auf Varianzhomogenität der Items zu Aspekten und Ausgängen von Integration für Vorstände und Mitglieder

\begin{tabular}{|l|l|}
\hline Item & Sig, \\
\hline Gut Deutsch sprechen & $\mathbf{0 , 0 0 3}$ \\
\hline Anerkennung wichtiger Werte & 0,697 \\
\hline Chancen zur Teilnahme an der Gesellschaft & 0,627 \\
\hline Der Wille, an der Gesellschaft teilzunehmen & 0,922 \\
\hline Kontakte zwischen Menschen verschiedener Kulturen & 0,643 \\
\hline $\begin{array}{l}\text { Mitgliedschaft/ } \\
\text { Engagement in Organisationen }\end{array}$ & 0,275 \\
\hline Anerkennung durch Andere & 0,347 \\
\hline Vorhandensein eines Zugehörigkeitsgefühls & 0,620 \\
\hline Gleiche Rechte für Alle & 0,535 \\
\hline Bildungsstand & $\mathbf{0 , 0 3 6}$ \\
\hline Berufliche Stellung & 0,451 \\
\hline Zugang zum Wohnungsmarkt & 0,819 \\
\hline Zufriedenheit mit beruflicher und sozialer Situation & $\mathbf{0 , 0 2 2}$ \\
\hline $\begin{array}{l}\text { Wenn Zuwanderer ihre Kultur beibehalten möchten, } \\
\text { sollten sie unter sich bleiben }\end{array}$ & 0,270 \\
\hline $\begin{array}{l}\text { Es wäre gut, wenn alle Zuwanderer in Deutschland } \\
\text { ihre Kulturen beibehielten }\end{array}$ & 0,974 \\
\hline $\begin{array}{l}\text { Menschen, die nach Deutschland kommen, sollten ihr } \\
\text { Verhalten der deutschen Kultur anpassen }\end{array}$ & 0,766 \\
\hline $\begin{array}{l}\text { Zuwanderer sollten sowohl in ihre } \\
\text { Herkunftsgesellschaft als auch in die deutsche } \\
\text { Gesellschaft eingebunden sein }\end{array}$ & 0,339 \\
\hline Q & \\
\hline
\end{tabular}

Quelle: Eigene Darstellung. 
Tabelle 36: Levene-Test auf Varianzhomogenität der Items zu Aspekten und Ausgängen von Integration für Migrantinnen und Migranten erster und zweiter Generation

\begin{tabular}{|l|l|}
\hline Item & Sig, \\
\hline Gut Deutsch sprechen & 0,204 \\
\hline Anerkennung wichtiger Werte & $\mathbf{0 , 0 1 3}$ \\
\hline Chancen zur Teilnahme an der Gesellschaft & 0,093 \\
\hline Der Wille, an der Gesellschaft teilzunehmen & 0,381 \\
\hline Kontakte zwischen Menschen verschiedener Kulturen & 0,436 \\
\hline $\begin{array}{l}\text { Mitgliedschaft/ } \\
\text { Engagement in Organisationen }\end{array}$ & 0,490 \\
\hline Anerkennung durch Andere & 0,509 \\
\hline Vorhandensein eines Zugehörigkeitsgefühls & 0,405 \\
\hline Gleiche Rechte für Alle & 0,309 \\
\hline Bildungsstand & 0,803 \\
\hline Berufliche Stellung & 0,985 \\
\hline Zugang zum Wohnungsmarkt & 0,252 \\
\hline Zufriedenheit mit beruflicher und sozialer Situation & 0,769 \\
\hline $\begin{array}{l}\text { Wenn Zuwanderer ihre Kultur beibehalten möchten, } \\
\text { sollten sie unter sich bleiben }\end{array}$ & 0,306 \\
\hline $\begin{array}{l}\text { Es wäre gut, wenn alle Zuwanderer in Deutschland } \\
\text { ihre Kulturen beibehielten }\end{array}$ & 0,210 \\
\hline $\begin{array}{l}\text { Menschen, die nach Deutschland kommen, sollten ihr } \\
\text { Verhalten der deutschen Kultur anpassen }\end{array}$ & 0,523 \\
\hline $\begin{array}{l}\text { Zuwanderer sollten sowohl in ihre } \\
\text { Herkunftsgesellschaft als auch in die deutsche } \\
\text { Gesellschaft eingebunden sein }\end{array}$ & 0,738 \\
\hline Q & \\
\hline
\end{tabular}

Quelle: Eigene Darstellung. 
Tabelle 37: Leitfaden für die explorativen Interviews

\begin{tabular}{|c|c|}
\hline Block & Fragen \\
\hline Einstieg & $\begin{array}{l}\text { - Bitte stellen Sie sich mit ihrer Funktion im Verein und den } \\
\text { Verein selbst vor. }\end{array}$ \\
\hline Vereinskultur & $\begin{array}{l}\text { - Welche Ziele verfolgt Ihr Verein? } \\
\text { - Sind diese erreichbar? Werden sie von den } \\
\text { Vereinsmitgliedern unterstützt? } \\
\text { - Haben Sie den Eindruck, dass sich alle Mitglieder zum } \\
\text { Verein zugehörig fühlen und sich aktiv ins Vereinsleben } \\
\text { einbringen? } \\
\text { - Wie gestaltet sich Partizipation in Ihrem Verein } \\
\text { (Mitarbeiter/Mitglieder)? Gibt es genügend freiwilliges } \\
\text { Engagement? } \\
\text { Wie schätzen Sie die Öffentlichkeitsarbeit Ihres Vereins ein? } \\
\text { Kann das freiwillige Engagement dadurch gesteigert werden? }\end{array}$ \\
\hline $\begin{array}{l}\text { Veränderungsprozesse } \\
\text { und Bedarfe }\end{array}$ & $\begin{array}{l}\text { Wie hoch schätzen Sie den Anteil an Migrantinnen und } \\
\text { Migranten im Verein ein (in Bezug auf Mitglieder und } \\
\text { Funktionäre)? } \\
\text { - Hat sich die Anzahl an Vereinsmitgliedern und -funktionären } \\
\text { mit Migrationshintergrund nach Ihrer Einschätzung im Laufe } \\
\text { der letzten Jahre verändert? Woran kann das liegen? } \\
\text { - Haben Sie den Eindruck, dass sich durch Menschen mit } \\
\text { Migrationshintergrund die Bedarfe im Verein ändern? } \\
\text { Welche Bedarfe sehen Sie auf Seiten des Vereins/der } \\
\text { Mitglieder? }\end{array}$ \\
\hline Umgang mit Vielfalt & $\begin{array}{l}\text { - Können Sie Konflikte beobachten, die durch kulturelle } \\
\text { Unterschiedlichkeit hervorgerufen werden? } \\
\text { - Sehen Sie Möglichkeiten, diese Konflikte zu reduzieren? } \\
\text { - Wird in Ihrem Verein über diskriminierungsspezifische } \\
\text { Aspekte geredet? }\end{array}$ \\
\hline $\begin{array}{l}\text { Integrationsprojekte } \\
\text { des Vereins }\end{array}$ & $\begin{array}{l}\text { - Hat ihr Verein spezielle Angebote für Geflüchtete und für } \\
\text { - } \text { Personen mit Migrationshintergrund? } \\
\text { - Seit wann / Dauer, bis Umsetzung erfolgt / } \\
\text { Herausforderungen / Erfolge } \\
\text { - Finanzierung/ nützliche Kooperationen } \\
\text { - Wie werden die beabsichtigten Wirkungen der Projekte } \\
\text { überprüft? } \\
\text { - Was müsste Ihrer Meinung nach gegeben sein, damit die } \\
\text { Integration in und durch Sport erfolgreich ist? }\end{array}$ \\
\hline
\end{tabular}


- Wenn nein: Wieso nicht? Könnten Sie sich vorstellen, sich in Zukunft dem Thema Integration mehr zu widmen?

Verständnis von

Integration
- Was bedeutet Integration in Ihrem Sportverein?

- Wo sehen Sie Stärken/Schwächen Ihres Vereins hinsichtlich der Integrationsarbeit? Wo bestehen Weiterentwicklungsbedarfe?

- Was bedeutet Integration für Sie persönlich?

Quelle: Eigene Darstellung. 
Tabelle 38: Ergebnisse des U-Tests der Bewertung von Aspekten und Zielen von Integration von Personen mit europäischem und außereuropäischem Migrationshintergrund

\begin{tabular}{|c|c|c|c|}
\hline Item & $\begin{array}{l}\text { Mittlerer } \\
\text { Rang } \\
\text { europäischer } \\
\text { MGH }\end{array}$ & $\begin{array}{l}\text { Mittlerer Rang } \\
\text { außereuropäisch } \\
\text { er MGH }\end{array}$ & $\begin{array}{l}\text { U-Test } \\
(\mathrm{z})\end{array}$ \\
\hline Gute Deutschkenntnisse & 38,20 & 28,50 & $-2,127 *$ \\
\hline Anerkennung wichtiger Werte & 37,01 & 30,05 & $-1,629$ \\
\hline $\begin{array}{l}\text { Chancen zur Teilnahme an der } \\
\text { Gesellschaft }\end{array}$ & 34,59 & 33,22 & $-0,333$ \\
\hline $\begin{array}{l}\text { Der Wille, an der Gesellschaft } \\
\text { teilzunehmen }\end{array}$ & 37,34 & 29,62 & $-1,900$ \\
\hline $\begin{array}{l}\text { Kontakte zwischen Menschen } \\
\text { verschiedener Kulturen }\end{array}$ & 37,75 & 29,09 & $-2,051 *$ \\
\hline $\begin{array}{l}\text { Mitgliedschaft/ } \\
\text { Engagement in Organisationen }\end{array}$ & 34,76 & 31,90 & $-0,630$ \\
\hline Anerkennung durch Andere & 34,00 & 32,86 & $-0,253$ \\
\hline $\begin{array}{l}\text { Vorhandensein eines } \\
\text { Zugehörigkeitsgefühls }\end{array}$ & 34,84 & 32,90 & $-0,451$ \\
\hline Gleiche Rechte für Alle & 33,53 & 34,62 & $-0,314$ \\
\hline Bildungsstand & 36,63 & 30,55 & $-1,311$ \\
\hline Berufliche Stellung & 35,63 & 31,86 & $-0,821$ \\
\hline Zugang zum Wohnungsmarkt & 33,91 & 34,12 & $-0,046$ \\
\hline $\begin{array}{l}\text { Zufriedenheit mit beruflicher und } \\
\text { sozialer Situation }\end{array}$ & 34,82 & 32,93 &,- 426 \\
\hline $\begin{array}{l}\text { Wenn Zuwanderer ihre Kultur } \\
\text { beibehalten möchten, sollten sie unter } \\
\text { sich bleiben }\end{array}$ & 35,19 & 32,34 & $-0,645$ \\
\hline $\begin{array}{l}\text { Es wäre gut, wenn alle Zuwanderer in } \\
\text { Deutschland ihre Kulturen beibehielten }\end{array}$ & 36,41 & 30,64 & $-1,292$ \\
\hline $\begin{array}{l}\text { Menschen, die nach Deutschland } \\
\text { kommen, sollten ihr Verhalten der } \\
\text { deutschen Kultur anpassen }\end{array}$ & 36,74 & 31,48 & $-1,157$ \\
\hline $\begin{array}{l}\text { Zuwanderer sollten sowohl in ihre } \\
\text { Herkunftsgesellschaft als auch in die } \\
\text { deutsche Gesellschaft eingebunden sein }\end{array}$ & 36,74 & 31,48 & $-1,210$ \\
\hline
\end{tabular}


Tabelle 39: Zusammenhänge zwischen Aspekten von Integration anhand von Spearman's rho

\begin{tabular}{|c|c|c|c|c|c|c|c|c|c|c|c|c|c|}
\hline Item & $1^{29}$ & 2 & 3 & 4 & 5 & 6 & 7 & 8 & 9 & 10 & 11 & 12 & 13 \\
\hline $\begin{array}{l}\text { Gute } \\
\text { Deutschkenntnisse (1) }\end{array}$ & 1,000 & $0,426 * *$ & $0,241 * *$ & $0,301 * *$ & 0,047 & $0,100^{*}$ & $0,225^{* *}$ & 0,084 & $0,102 *$ & $0,264 * *$ & $0,171 * *$ & $0,198 * *$ & $0,206 * *$ \\
\hline $\begin{array}{l}\text { Anerkennung } \\
\text { wichtiger Werte (2) }\end{array}$ & $0,426 * *$ & 1,000 & $0,292 * *$ & $0,419 * *$ & $0,150 * *$ & $0,100 *$ & $0,190 * *$ & $0,201 * *$ & $0,161 * *$ & $0,163 * *$ & 0,091 & $0,148 * *$ & $0,198^{* * *}$ \\
\hline $\begin{array}{l}\text { Chancen zur } \\
\text { Teilnahme an der } \\
\text { Gesellschaft (3) }\end{array}$ & $0,241 * *$ & $0,292 * *$ & 1,000 & $0,343 * *$ & $0,386 * *$ & $0,118 *$ & $0,328 * *$ & $0,368 * *$ & $0,352 * *$ & $0,110 *$ & 0,010 & $0,462 * *$ & $0,336 * *$ \\
\hline $\begin{array}{l}\text { Der Wille, an der } \\
\text { Gesellschaft } \\
\text { teilzunehmen (4) }\end{array}$ & $\mathbf{0 , 3 0 1 * *}$ & $0,419 * *$ & $0,343 * *$ & 1,000 & $0,354 * *$ & $0,142 * *$ & $0,151 * *$ & $0,308^{* *}$ & $0,188 * *$ & 0,064 & 0,008 & $0,130 * *$ & $0,230 * *$ \\
\hline $\begin{array}{l}\text { Kontakte zwischen } \\
\text { Menschen } \\
\text { verschiedener } \\
\text { Kulturen (5) }\end{array}$ & 0,047 & $0,150 * *$ & $0,386 * *$ & $0,354^{* *}$ & 1,000 & $0,336 * *$ & $0,372 * *$ & $0,426 * *$ & $0,264^{* *}$ & 0,085 & 0,041 & $0,245^{* *}$ & $0,246 * *$ \\
\hline $\begin{array}{l}\text { Mitgliedschaft/Engag } \\
\text { ement in } \\
\text { Organisationen (6) }\end{array}$ & $0,100 *$ & $0,100 *$ & $0,118 *$ & $0,142 * *$ & $0,336 * *$ & 1,000 & $0,402 * *$ & $0,294 * *$ & $0,172 * *$ & $0,222 * *$ & $0,208 * *$ & $0,155^{* * *}$ & $0,143 * *$ \\
\hline $\begin{array}{l}\text { Anerkennung durch } \\
\text { andere (7) }\end{array}$ & $0,225^{* *}$ & $0,190 * *$ & $0,328 * *$ & $0,151 * *$ & $0,372 * *$ & $0,402 * *$ & 1,000 & $0,501 * *$ & $0,291 * *$ & $0,287 * *$ & $0,242 * *$ & $0,396 * *$ & $0,287 * *$ \\
\hline $\begin{array}{l}\text { Vorhandensein eines } \\
\text { Zugehörigkeitsgefühls } \\
\text { (8) }\end{array}$ & 0,084 & $0,201 * *$ & $0,368^{* * *}$ & $0,308^{* *}$ & $0,426 * *$ & $0,294 * *$ & $0,501 * *$ & 1,000 & $0,406^{* *}$ & $0,160 * *$ & 0,093 & $0,366^{* * *}$ & $0,344 * *$ \\
\hline $\begin{array}{l}\text { Gleiche Rechte für } \\
\text { alle (9) }\end{array}$ & $0,102 *$ & $0,161 * *$ & $0,352 * *$ & $0,188 * *$ & $0,264 * *$ & $0,172 * *$ & $0,291 * *$ & $0,406 * *$ & 1,000 & 0,071 & $-0,006$ & $0,300 * *$ & $0,259 * *$ \\
\hline Bildungsstand (10) & $0,264 * *$ & $0,163 * *$ & $0,110 *$ & 0,064 & 0,085 & $0,222 * *$ & $0,287 * *$ & $0,160 * *$ & 0,071 & 1,000 & $0,666 * *$ & $0,299 * *$ & $0,256^{* *}$ \\
\hline
\end{tabular}

\footnotetext{
${ }^{29}$ Aus Platzgründen werden die Items hier anhand der Nummerierung in der ersten Spalte der Tabelle dargestellt.
} 


\begin{tabular}{|c|c|c|c|c|c|c|c|c|c|c|c|c|c|}
\hline $\begin{array}{l}\text { Berufliche Stellung } \\
\text { (11) }\end{array}$ & $0,171 * *$ & 0,091 & 0,010 & 0,008 & 0,041 & $0,208 * *$ & $0,242 * *$ & 0,093 & $-0,006$ & $0,666 * *$ & 1,000 & $0,287 * *$ & $0,269 *$ \\
\hline $\begin{array}{l}\text { Zugang zum } \\
\text { Wohnungsmarkt (12) }\end{array}$ & $0,198 * *$ & $0,148 * *$ & $0,462 * *$ & $0,130 * *$ & $0,245 * *$ & $0,155 * *$ & $0,396 * *$ & $0,366 * *$ & $\mathbf{0 , 3 0 0 * *}$ & $0,299 * *$ & $0,287 * *$ & 1,000 & $0,617 *$ \\
\hline $\begin{array}{l}\text { Zufriedenheit mit } \\
\text { beruflicher und } \\
\text { sozialer Situation (13) }\end{array}$ & $0,206 * *$ & $0,198 * *$ & $0,336 * *$ & $0,230 * *$ & $0,246 * *$ & $0,143 * *$ & $0,287 * *$ & $0,344 * *$ & $0,259 * *$ & $0,256 * *$ & $0,269 * *$ & $0,617^{* *}$ & 1,000 \\
\hline
\end{tabular}

Quelle: Eigene Darstellung, *p $<0,05 ;{ }^{* *} \mathrm{p}<0,01$ 
